\title{
A System-Level Approach to Single-Molecule Live-Cell Fluorescence Microscopy
}

\author{
Oliver L. J. Harriman
}

A Thesis Submitted in Partial Fulfilment of the Requirements for the Degree of Doctor of Philosophy at the University of Oxford

\author{
Hertford College \\ University of Oxford \\ Trinity Term 2013
}




\begin{abstract}
In this work a system-level approach was taken to the single-molecule fluorescence microscopy of living cells. This primarily involved the unification of relevant information within appropriately structured artefacts that were used to inform and enhance experimentation.

Initially the diversity of emerging single-molecule techniques was reviewed and presented with a novel article structure to suit the purpose of designing an experiment (Harriman and Leake 2011). Techniques were grouped by the type of information they could access, rather than the standard organisation centred on the techniques themselves.

A bespoke microscope was conceived and built with reference to knowledge and tools from the fields of Architecture and Systems-Engineering. The microscope layout would enable multiple experiment types through independent control of multiple illumination beams. A technique was developed enabling the prescription of evanescent field penetration depth for each incident beam.

The various empirical and theoretical results that are used to understand and modify a microscopy experiment were integrated into an internally consistent simulation model (Harriman and Leake. 2013). This was used to inform the selection of experimental components and parameters and ultimately acquire higher data quality as measured by functions such as signal-to-noise ratio (SNR).

The combined experimental system of microscope and simulation model was applied in two live-cell investigations. In Escherichia coli, the spatial distribution of membrane bound proteins was investigated and a novel technique was applied to the
\end{abstract}


analysis of colocalisation. Results indicate that NADH dehydrogenase and ATP synthase follow uncorrelated trajectories. This supports the hypothesis of spatial decoupling of molecules that energise the membrane and molecules that use membrane energy

In human carcinoma cells, the mechanism of ligand-receptor binding was investigated. Data was collected prior to and periodically after the addition of ligands, and fluorescence images were acquired of both ligands and receptors. Analyses based on single particle tracking are currently being carried out by a collaborator to extract information on stoichiometry and dynamics at the single-molecule level. 


\section{Publications Arising from Doctoral Research}

Harriman, Oliver, and Mark Leake. "Advanced Multidimensional Optics to Investigate Biological Complexity at the Single-molecule Level in Living, Functional Cells." Biophysical Journal 98.3 (2010): 587a.

Harriman OLJ \& Leake MC. Single-molecule experimentation in biological physics: exploring the living component of soft condensed matter one molecule at a time. J. Phys.: Condens. Matter (2011), 23, 503101.

Llorente Garcia, Isabel, Oliver LJ Harriman, and Mark C. Leake. "Sub-Millisecond Single-molecule Fluorescence Imaging Combined with Dual Optical Tweezers on DNA Tethers." Biophysical Journal 102.3 (2012): 180a.

Harriman OLJ \& Leake MC. A System Level Approach to Single-molecule Live-Cell Fluorescence Microscopy. infocus Magazine / Royal Microscopical Society (2013), 30.

Llorente Garcia, Isabel et al. "Localization dynamics of oxidative phosphorylation machinery in vivo indicate electron transport and proton-motive force delocalization". Submitted to Nature Communications on 16/09/2013. 


\section{Declaration}

The work in this thesis was undertaken at the University of Oxford; in the Clarendon Laboratory, Department of Physics. Work was performed from October 2009 to September 2013 under the supervision of Professor Mark Christian Leake. All work in this thesis is my own unless stated and has not been submitted for a degree at this or any other university.

The following specific contributions were made by my collaborators. Dr Isabel Llorente Garcia integrated infrared laser tweezers into the microscope (section 2.1.3) and wrote the image analysis software applied to data from the carcinoma cell line (section 3.2.2). Dr Tchern Lenn and Dr Heiko Erhardt generated the E. coli cell lines (imaged in sections 1.2.1 and 3.1), and Dr Charlotte Fournier generated the carcinoma cell line and carried out its initial analyses (section 3.2.1). 


\section{Acknowledgements}

I thank my supervisor, Professor Mark Christian Leake, for the freedom he allowed me in developing and pursuing my interests. I am also grateful for the opportunities he presented to me, his responsiveness, and his down to earth nature. I thank Dr Ian Dobbie for his early contributions of opinion and enthusiasm; Dr Isabel Llorente Garcia for her scientific interest and spirit; and Dr Charlotte Fournier for her friendly and educating collaboration. I also thank Dr Alex Robson for his impressive demonstration of resilience.

More than ever I am grateful for the support and inspiration from my close

family; my brothers Andre and Lucian, and parents Barbara and Ed. I hold in honour my fiancée Sakura, for leading the way with love, humour, and determination. 


\section{Contents}

1 CONTEXT OF THE Microscopy System 1

1.1 Single-Molecule Experimentation in Biological Physics ............................2

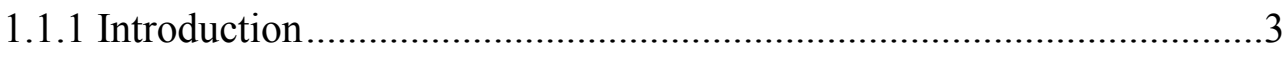

1.1.2 Single-Molecule Experimentation .....................................................

1.1.2.1 Historical Background …...............................................5

1.1.2.2 Single-Molecule Systems........................................... 9

1.1.2.3 What Characterizes a Living System? ............................13

1.1.2.4 The Central Importance of Fluorescence Techniques .......14

1.1.3 The Detection of Single-Molecules ...................................................19

1.1.3.1 Is a Single-Molecule Present (or Absent)? ......................20

1.1.3.2 How Many Single-Molecules are there?.........................24

1.1.3.3 What is the Structure of a Single-Molecule? ...................28

1.1.3.4 Can we Determine the Conformational State of a Single-

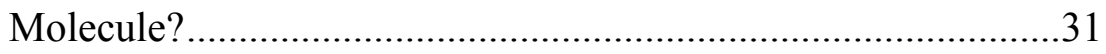

1.1.4 Investigating Intermolecular Details ............................................33

1.1.4.1 The Orientation of a Single Molecule..............................33 
1.1.4.2 Single-Molecule Spatial Location..........................................34

1.1.4.3 The Relative Position of Many Single-Molecules ...............36

1.1.4.4 Single-Molecule Translational Activity...............................39

1.1.4.5 Observing Molecular Binding and Unbinding ....................41

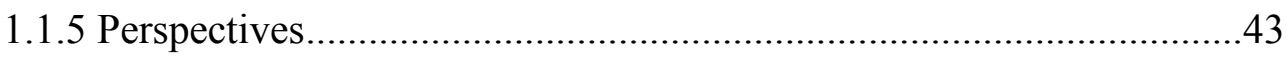

1.2 Preliminary Work and Direction of Research..............................................45

1.2.1 Preliminary Experimentation ............................................................45

1.2.2 The Challenge of Single-Molecule Live-Cell Fluorescence Microscopy50

1.2.3 Vision of a System-Level Approach to Single-Molecule Live-Cell Fluorescence Microscopy .......................................................................53

2 Creation Of THE Microscopy System 56

2.1 Development of the Bespoke Microscope ………..........................................57

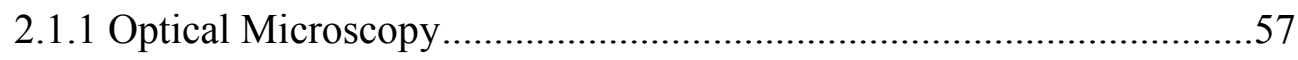

2.1.1.1 The Compound Optical Microscope .....................................57

2.1.1.2 Fluorescence Microscopy ……………………………........60

2.1.2 Architecture Development ................................................................

2.1.2.1 The Engineering of Systems ............................................73

2.1.2.2 Purpose of the Microscope.................................................. 
2.1.2.3 Optical Patterns .........................................................80

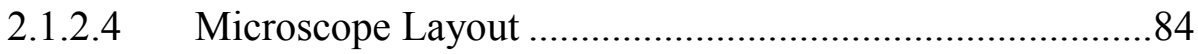

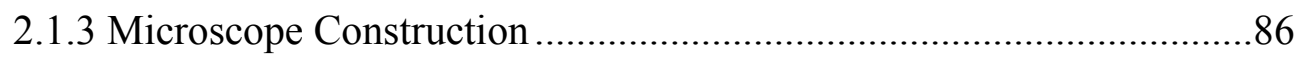

2.1.3.1 Excitation Path Development ......................................92

2.1.3.2 Alignment and Calibration........................................ 100

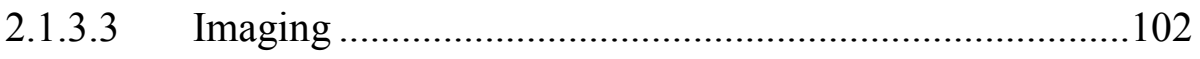

2.2 Model-Aided Experimentation ...............................................................108

2.2.1 Model-Instructed Control of Illumination .........................................108

2.2.1.1 Prescriptive Polarisation-Based Power Distribution........108

2.2.1.2 Spectral Band Selection for Two-Colour Excitation .......112

2.2.1.3 Reverse Ray Tracing to Inform Beam Steering ..............117

2.2.1.3.1 Modelling of the Objective Lens..........................117

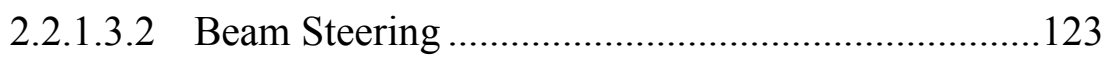

2.2.1.4 Evanescent Field Specification for Highest Membrane-

Cytoplasm Excitation Ratio .............................................. 124

2.2.2 Model-assisted Light Quantification............................................127

2.2.2.1 Mapping of the Excitation Field in Three-Dimensions ...127

2.2.2.2 Single-Molecule Brightness .......................................130

2.2.2.3 Measurement of Photobleaching...................................132

viii 
2.2.3 Experiment Simulation for Improved Imaging Data

2.2.3.1 Modelling the Whole Experimental System .....................135

2.2.3.2 The Model Implementation.................................................139

3 APPliCATIONS OF THE MiCROSCOPY SYSTEM 153

3.1 Two-Colour TIRFM to Illuminate the Spatial Organisation OXPHOS

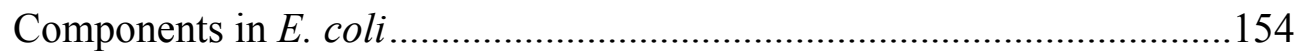

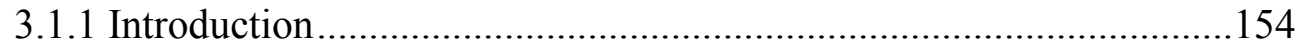

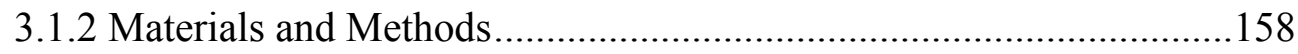

3.1.2.1 Experimental Procedure ....................................................158

3.1.2.2 Generation of a Spatial Transform for Equating Positions in the Red and Green Images ...........................................................161

3.1.2.3 Optimising Imaging Parameters ………………………....162

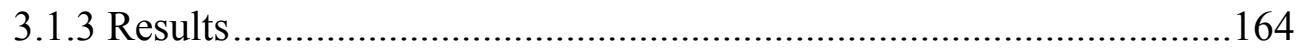

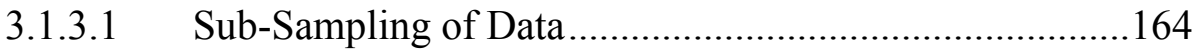

3.1.3.2 Distinguishing Signal and Noise.........................................165

3.1.3.2.1 Distinguishing Cell Types through Total Cell Intensity 165

3.1.3.2.2 Distinguishing Cell Types through Inspection.........166

3.1.3.3 Computation of Fluorescence Correlation .......................170 
3.1.3.3.1 Low pass Spatial Filtering of Images 170

3.1.3.3.2 Colour Combination for Maps of Channel Correlation 172

3.1.3.3.3 A Quantitative Measure of Correlation 174

3.1.3.3.4 Simulated Random cells 175

3.1.3.3.5 Fluorescence Correlation 176

3.1.3.3.6 Summation of Binarised Images 180

3.1.3.3.7 Novel Correlation Analysis Technique 181

3.1.3.3.8 Correlation of Red and Green Channel Displaced in Time 186

3.1.3.3.9 The Correlation of Each Channel with Itself Displaced In Time 187

3.1.4 Summary and Conclusions 189

3.2 The Binding and Dynamics of Epidermal Growth Factor and Epidermal Growth Factor Receptor in Cancerous Human Epithelial Cells 193

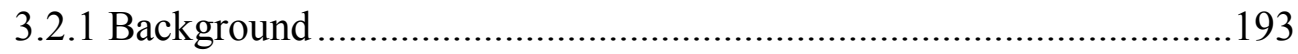

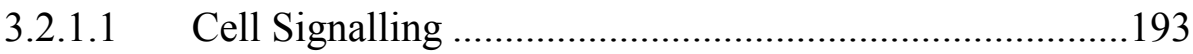

3.2.1.2 The Role of the EGFR in Development and Cancer........195

3.2.1.3 Generation of Cell Lines for Fluorescence Imaging........198

3.2.2 Single-Molecule TIRF Imaging of the Cell Lines ..........................202 
4.1 Reflections on Development of the Microscope.

4.2 Reflections on Simulation of the Experimental System 216

5 BACK MATTER

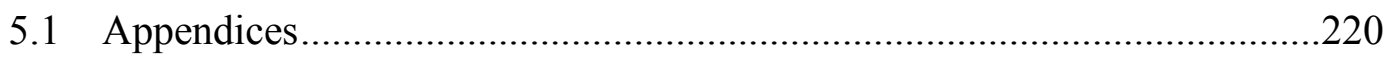

5.1.1 Technique for Micrometer Incident Angle Control ..........................220

5.1.2 Protocol for In Vitro Fluorescent Protein Sample Preparation ..........223

5.1.3 E. coli Results: Average Binarised Images and Correlation against Time Displacement Plots................................................................224

5.1.3.1 Simulated Random Strain ..........................................224

5.1.3.2 Parental Strain ...........................................................225

5.1.3.3 mCherry-nuoF/ATPase-GFP Strain .............................226

5.1.3.4 GFP-nuoF/mCherry-sdhC Strain ................................227

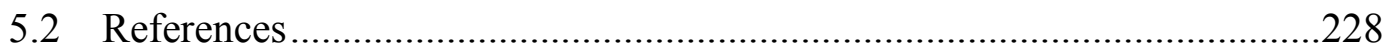




\section{CONTEXT OF THE MICROSCOPY SYSTEM}




\subsection{Single-Molecule Experimentation in Biological Physics}

The soft matter of biological systems consists of mesoscopic length scale building blocks, composed of a variety of different types of biological molecule. Most single biological molecules are so small that 1 billion would fit on the full-stop at the end of this sentence, but collectively they carry out the vital activities in living cells whose length scale is at least three orders of magnitude greater. Typically, the number of molecules involved in any given cellular process at any one time is relatively small and so real physiological events may often be dominated by stochastics and fluctuation behaviour at levels comparable to thermal noise, and are generally heterogeneous in nature. This challenging combination of heterogeneity and stochasticity is best investigated experimentally at the level of single molecules, as opposed to more conventional bulk ensemble-average techniques. In recent years, the use of such molecular experimental approaches has become significantly more widespread in research laboratories around the world. In this review I discuss recent biological physics experimental approaches which can be applied to investigate the living component of soft condensed matter to a precision of single molecules. 


\subsubsection{INTRODUCTION}

Single-molecule experimentation (SME) is a banner for approaches that measure or manipulate individual molecules that may be in the context of larger systems, for example in actual complex soft matter of living biological cells, or in highly reduced environments such as single-molecule assays in vitro in which perhaps only the one type of purified, isolated single-molecule in present. SME has come into fruition over the last twenty years and represents a powerful complement to the predominant approaches using ensemble-averaging. Through measurement of previously unresolved characteristics, the range of new techniques have permitted several familiar molecules to become significantly better understood. These techniques have been applied particularly enthusiastically in biology, and have revealed some profound new aspects of life at the molecular level.

Articles reviewing SME have been published from various perspectives. A recent, broadly encompassing review (Claridge, Schwartz et al. 2011) gives coherent graphical representations of the basic classes of techniques and associated discoveries. In it, the field of SME is decomposed into techniques that primarily use electrons, photons, or force to probe or manipulate. Other reviews have focused on the ubiquitous approaches of fluorescence microscopy (Lichtman and Conchello 2005), and force spectroscopy (Neuman and Nagy 2008). There are also some extensive reviews written from a biophysics perspective (Ritort 2006; Deniz, Mukhopadhyay et al. 2008), and emphasizing the theoretical character of SME (Kapanidis and Strick 2009).

My review here assumes that the reader has some awareness of many of the basic experimental techniques that have been ultimately implemented in SME, and looks at 
SME with respect to what it may achieve conceptually, in terms of the raw information it provides in light of the underlying physical nature of the living soft condensed matter. Techniques are presented relating to a progression of abstract measurements or manipulations that one might wish to make and, where appropriate, ensemble-average techniques are referred to. Many techniques could conceivably provide answers to each question, and it is hoped that concurrent presentation of the various approaches may prove useful to the experimentalist. For each case, the physical and chemical specifics of the molecule in question and its surrounding environment should be taken into account since these qualities are ultimately what is of interest, and may predetermine the suitable technique (Walter, Huang et al. 2008).

In this review SME is presented as a route to discovering how the structure and behaviour of living soft condensed matter can be explained and quantitatively described. The difference between considering living and non-living soft condensed matter is primarily a conceptual one of functionality, involving paradigms for systems level complexes and interaction, such as those of chemical networks. In order that the physicochemical characteristics of biomolecules can be experimentally determined, the majority of biophysics SME at present is carried out on molecules in vitro, isolated from confounding factors of their native environment. However, there have been recent successes involving SME in the true physiological context of the living cell, and such directions of research are likely to become significantly more widespread in the next few years.

This review starts with a discussion of the key qualities of SME in relation to other approaches to the investigation (and in some cases manipulation of molecules). 
Some historical context is given, highlighting advances leading to the realization of SME. The following section discusses the relation between single molecules and their environments. The bulk of the article is an exploration of techniques capable of answering the aforementioned focus questions. The questions are loosely ordered by increasing conceptual sophistication of the associated information gained, or manipulation achieved. The last section discusses the future of SME including commentary on observed trends and the combination of techniques.

\subsubsection{Single-MoleCUle EXPERIMENTATION}

\subsubsection{Historical Background}

The alternative to single-molecule investigation, measuring mean properties of ensembles of molecules, has led to the vast majority of current biochemistry knowledge. However, measurement of properties of individual molecules, as opposed to ensembles, enables conceptually new types of information to be obtained. Simply speaking, the mean value for a property of a system produced by a bulk ensemble average approach may be replaced by a probability distribution of values for that property of the system when a single-molecule approach is used. This is made possible by the direct observation of a property of one or many individual molecules. SME enables investigation when the number of molecules of interest is prohibitively small for ensemble measurements. SME can also be seen to subsume the capabilities of ensemble experimentation if the Ergodic

hypothesis is true for the system under study. This denotes that the average of a measured parameter over a "long time" (dependent on the system), and the average over 
the statistical ensemble, should be the same. Regardless of the applicability of the Ergodic hypothesis, SME has introduced a grand new set of capabilities.

Heterogeneity, both static and dynamic, is an intrinsic feature of biomolecules and their function. Statically heterogeneous molecules are those that do not play an active role in the interactions of interest of a biological system. In a standard ensemble investigative method, these molecules must be accounted for in analyses in order that their presence can be seen not to have given weight to the results. This step may however involve inaccurate approximation. Dynamic heterogeneity refers to inter-conversion of a subgroup of molecules in the system that occurs within the time scale of the observation. This dynamic variation will be lost in the bulk value gained by ensemble study as the stochastic activity of the numerous inter-converting molecules may, in all but some exception cases, be unsynchronized. In this case, a single-molecule technique may produce one of two types of result that bear further information on the dynamic heterogeneity. If the inter-conversion takes place at a rate that can be effectively sampled by the experimental temporal resolution, the property relating to discrete stages of activity will be discerned. In other words, variation in time of a property may be measured. If the process occurs more rapidly than the technique may resolve, the measured result may represent a weighted time average of the property when the molecule has explored a range of different states. As an example, one might be investigating a molecule's biochemical reaction pathway. The single-molecule approach might be to use fluorescence microscopy to record a video of the molecule in question and analyse this to produce a frequency histogram of its dwell times at each state. This 
may elucidate rare or transient states that might not have been observed with an ensemble method.

SME approaches have now become widely adopted, to the extent of there now being a wide range commercially available experimental apparatus available to perform such experiments compared to the situation ten or more years ago when the vast majority of devices were home-made. SME techniques (Figure 1) are being unleashed on exciting questions across science, particularly in understanding biological matter. In the last millennium a significant historical development in SME included the application of scanning probe methods, first utilised in scanning tunnelling microscopy (STM) to map surface topography (Binnig, Rohrer et al. 1982) in 1982, followed by its utility in nonbiological condensed matter physics for manipulating single atoms (Eigler and Schweizer 1990) in 1990. 1986 saw the invention of atomic force microscopy (AFM) (Binnig, Quate et al. 1986) and the trapping of dielectric particles with a light beam (Ashkin, Dziedzic et al. 1986). In 1991, far-field light microscopy was used to track gold nanoparticles attached to DNA being transcribed by immobilized RNA polymerase, and the following year the elasticity of DNA was probed with magnetic and hydrodynamic force (Smith, Finzi et al. 1992). In 1991 the first detection of single-molecule fluorescence in diffusion was achieved (Soper, Shera et al. 1991) and in 1996 the structure of Green Fluorescent Protein (GFP) was solved (Yang, Moss et al. 1996). Single-molecule detection was achieved via surface-enhanced Raman scattering (SERS) in 1997 (Kneipp, Wang et al. 1997) and in 1998 far-field microscopy was used to resolve numerous single-molecules, breaking the Abbe diffraction limit, in three dimensions (van Oijen 1998). Comprehensive reviews of the early histories of single-molecule approaches 
exist, such as those on single-molecule spectroscopy (Moerner 2002) and DNA mechanics (Bustamante, Bryant et al. 2003).

In the first decade of the new millennium, tremendous progress was made including dramatic improvements of fluorophore, optical, and data analysis technologies. What now exists is a range of techniques (succinctly represented in (Claridge, Schwartz et al. 2011)) that have been aggressively applied in novel combinations, to a diverse range of molecules and environments. The methods have been modified to become more biocompatible and gain ever deeper access to the molecular mechanisms in live samples. Biological insight has been gained with relevance to a number of topics including protein folding, membrane proteins, molecular motors and DNA-protein interactions, including the methods by which the DNA code is ultimately read-off and converted into functional proteins via translation and transcription respectively. The frontiers of scientific knowledge and experimental capability are now being challenged ferociously. 


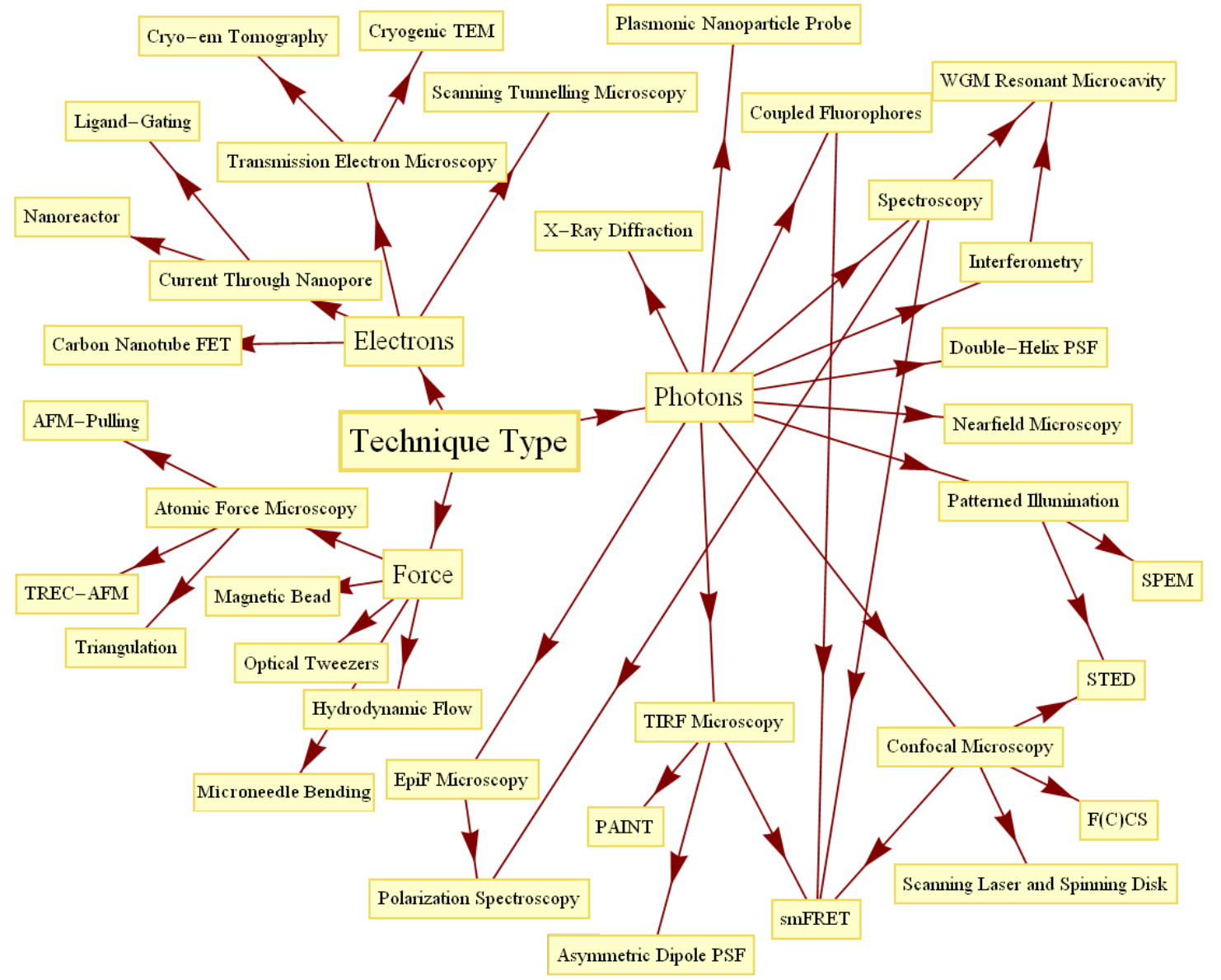

Figure 1 A technique-oriented network schematic of single-molecule experimentation.

\subsubsection{Single-Molecule Systems}

The purpose of the single-molecule experiment imposes specific expectations and requirements. In some cases the emphasis is on minimizing the perturbation of the molecular system of interest, while in other cases the fine control over environmental conditions is what allows the technique to ultimately be successful, and in many instances the ordering of the environment is the ultimate goal (for example, in nanotechnology). 
The focus of this review is on approaches that may conceivably enable one to gain specific information on biomolecules, in or out of their native contexts.

Where the effort is to characterize the molecules as independent entities, the default approach is to make measurements in the most reduced practicable environment. This may involve cryogenic temperatures, vacuums, and spatial constraint; to minimize phenomenon such as instrument noise, background artefacts, Brownian motion and contamination. At low temperatures high resolution optical methods can be applied and many more phenomena may be accessed (Moerner 2002).

In vitro methods allow for very specific physical characterizations of individual molecules with a broad range of probing techniques. Techniques can be used that might adversely affect the working order of a living cell. Small reconstituted systems of biomolecules may be composed in an attempt to reproduce molecular interactions and discover emergent behaviour that might be present in living cells (Sanders, Dallmann et al. 2010). The highly controlled and understood in vitro environment may make it more tractable to physically characterize the biomolecules and develop predictive, quantitative theories for their collective behaviour. This information can be considered in the interpretation of live-cell measurements and vice versa.

In order to discover the true activity of biomolecules in their native physiological conditions, one must observe living cells. While environmental complexity may preclude full physical and chemical understanding, observations of phenomenon may illuminate biological mechanisms that make life possible. This has proved to be technically highly challenging for reasons that will be discussed later in the review. Significant progress has however been made in the last five years, and some counter intuitive processes have 
been inferred. Imaging in time of a single-molecule in a live-cell is by its nature embracing the systems biology approach. The observed molecule is exposed to the numerous interdependent reaction networks as their activity changes to modify the organism's behaviour. Many molecules have been observed (including signalling molecules and transcription factors) whose activity is regulated by association with specific intracellular structures or by transport between sub compartments of the cell (Andrews, Harper et al. 2002). Analysis of the trajectories of individual molecules in the cell membrane has shown anomalous diffusion behaviour, a more hindered version to that associated with standard Brownian motion (Thompson 2002). The line between what are the intrinsic and extrinsic features of a single-molecule is blurred. Through its conformation, location and association within its environment, a single-molecule can be a local reporter of the functional groups, atoms, ions, electrostatic charges, and/or other sources of local fields in its immediate vicinity (Moerner 2002). For example the structural form a protein holds in water is intimately related to hydrophobicity. A molecule's characteristics should be considered as a function of both its identity and its environment (Figure 2). Viscosity has been determined by following the relaxation rate of DNA after end-to-end stretching and photoscission (Fisher, Ballenger et al. 2009). Solvent polarity and $\mathrm{pH}$ of an aqueous medium can affect fluorescent properties. Using ratiometric measurements, ion concentrations can be robustly calculated ( $\mathrm{O}^{\prime}$ Connor and Silver 2007).

A theme of cell biology is that there can be paradigmic similarity in the molecular mechanisms of cells across the diverse range of living species. This leads to the use of model organisms that can become well understood and are seen to give insight into a 
much broader range of species. An example that is a frequent subject of SME is the $E$. coli bacterium (cultivated strains of the commonly-used "K12" variant of E. coli can be grown easily and are well-adapted to the laboratory environment). The examination of such specimens and their constituents is currently being done according to two grand aspirations. The first is to understand cellular behaviour in a deterministic manner, based of biophysical analysis of constituent molecules. The second involves a more inclusive approach that sees complete understanding of the cell to be reliant on consideration of the entire dynamic interrelated system of molecules.

Soft condensed matter is defined as being characterized by the weak interactions between polyatomic constituents, by important thermal fluctuation effects, by mechanical softness and by a rich range of behaviours (Riste $\mathrm{T}$ 1990), and this can be said of biomatter. Molecules exist within a milieu of fluctuating interactions including electrostatic forces associated with charges and dipoles, transient hydrogen bonds, dispersion, hydrophobic and osmotic forces. The well-known self-assembled tessellating structures of soft condensed matter include micelles and membranes, both of which are prevalent in and enable biological systems to survive. An example of an area of active research is phase behaviour observed in the membrane (Simons and Ikonen 1997; Semrau, Idema et al. 2008; Deserno 2009; Semrau and Schmidt 2009). 


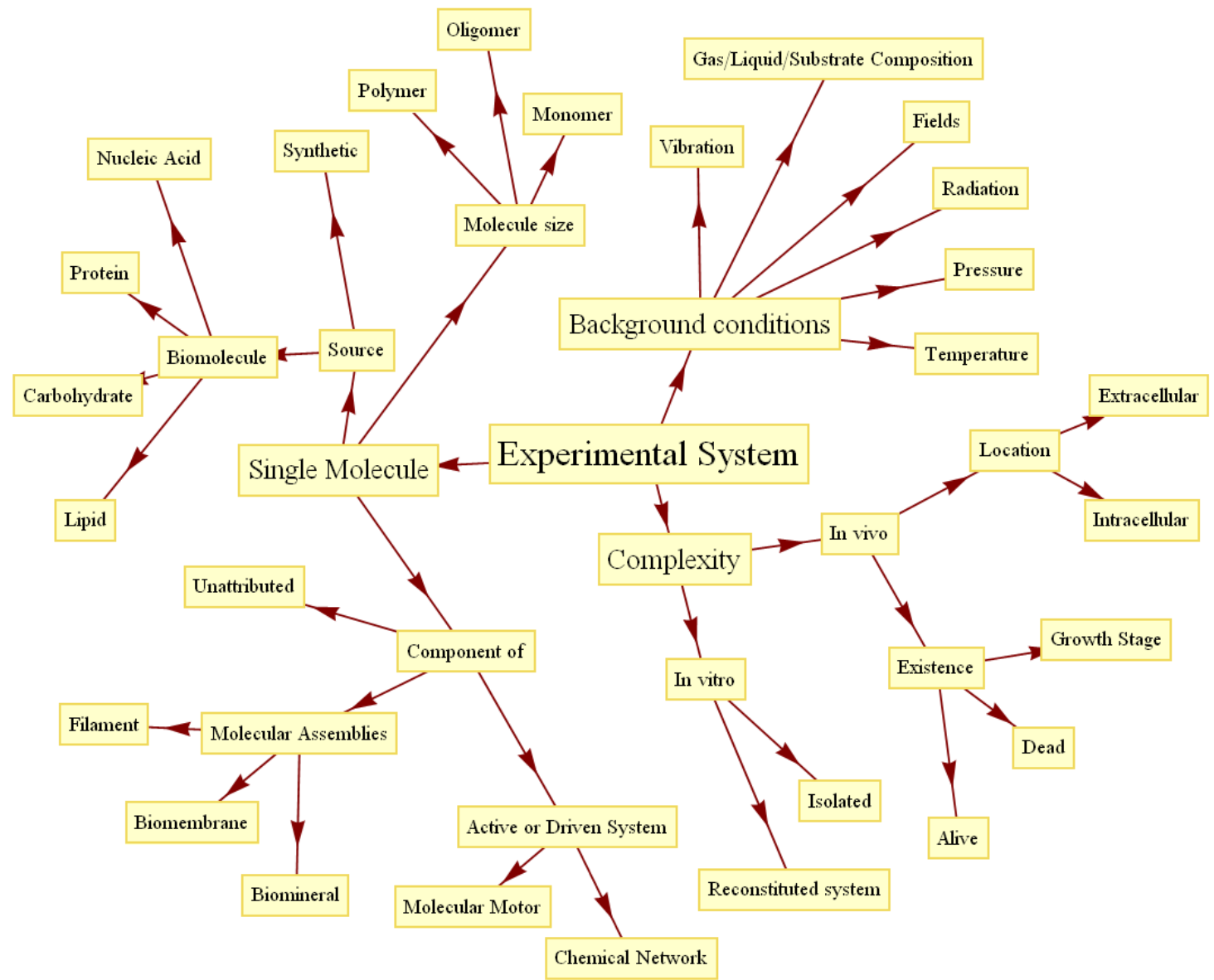

Figure 2 The molecule under study exists as part of a larger experimental system, with both a physical and functional context.

\subsubsection{What Characterizes a Living System?}

With consideration of a system that is living, new themes and interactions become relevant. Living systems are distinctive in their use of free energy to create order, essentially functioning as machines that, on a local level, generate negative entropy changes. Non-equilibrium states are osmotically maintained through pumping of ions by molecular machines. Energy is obtained from light and food and molecular machines use it to build structures, to power actuators, to actively transport molecules, to generate heat 
and light, and to regulate these processes. In their native environments, biomolecules have functional/logical significance as well as standard physical descriptions. A key aspect of biological systems is the specificity with which constituent molecules interact. Reaction rates are increased by enzymes with highly specific binding, transitions in macromolecules are sharpened by cooperativity, and biased random walks are attained based on the geometry of mechanisms (Nelson 2008). Manifold heterogeneity is a fundamental characteristic of life.

The physical single-molecule understanding we gain through SME may be multiple levels removed from the functional meaning that is of most relevance to the organism. Single-molecules may exist in a highly complex molecular system, in order to ultimately affect a basic mechanical property at the organism level, such as cellular stiffness (da Silva, Lautenschläger et al. 2010). Also, the nanometre length scale form of biomatter may only make sense in the light of the macroscale capabilities of the organism (Autumn, Sitti et al. 2002).

\subsubsection{The Central Importance of Fluorescence Techniques}

With the conjugation of a discernible label, a vast range of single-molecule approaches become available. Labels have been developed synthetically and from naturally expressed molecules such as the now ubiquitous GFP. Various classes of label exist including plasmonic nanoparticle probes, fluorescent labels, quantum dots and chemiluminescent labels (in which the excited state is populated via a coupled chemical reaction) (Haugland, Spence et al. 2005; McNamara, Gupta et al. 2006). Stimulated 
emission can also be employed in the imaging of non-fluorescent chromophores (Min, Lu et al. 2009).

Fluorescent probes are designed to localize within a specific region and or associate with a specific molecule. Fluorescence is used as a mechanism for high sensitivity measurement because of the inherent "Stokes shift". Emitted light can be isolated from the excitation light as it has a maximum intensity at a longer wavelength. Fluorescence enables detection of otherwise invisible molecules and the fluorophore may be re-excited many times to achieve a high signal within a given time. Fluorophores are characterized by a number of measurable properties including extinction coefficient, quantum yield, time to bleach (to half intensity at an excitation power that initially caused 1000 photons/second) (Shaner, Campbell et al. 2004).

The phenomena of quenching (loss of fluorescence) can be a result of short range interactions with other fluorophores and the molecular environment including binding to a target molecule. In quenching the fluorescence quantum yield is reduced without a change in the emission spectrum. Binding of a label to its target can reduce the fluorescent quantum yield and this can be used to monitor enzymatic cleavage (Haugland, Spence et al. 2005). Similarly, many fluorophores (that have a quenching effect on each other) may be attached to a molecule and an increase in brightness observed upon molecular cleavage. Another mechanism, Förster resonance energy transfer (FRET), is the non-radiative energy transfer between an acceptor-donor molecule pair that results from their dipole-dipole coupling when brought into close proximity (often the FRET pair of molecules are fluorescent and so some researchers refer to 
fluorescence resonance energy transfer synonymously). The visible result is that the emission peak of the fluorophore accepting energy increases in intensity.

Fluorescence based techniques are advantageous for live-cell measurement as they are relatively non-invasive, quantitative and can be recorded over "real-time", i.e. a time scale which is at least as fast as the biological process under investigation. The ambitious goal of fluorescence microscopy on live-cells is to be able to simultaneously discern multiple types of molecules in motion through time, in the three spatial dimensions, and in any region of the cell. Ideally one would be able to count and characterize the distribution of molecules in the cell and make inferences of the conformational state of each.

There exist significant challenges to the use of fluorescence for observations in living samples, indeed fluorescence imaging can have detrimental effects to the normal activity of a live-cell. An unintended consequence of fluorophore illumination can be the production of reactive oxygen species (ROS) which are known for their phototoxic effects (Dixit and Cyr 2003). Some fluorescence imaging techniques including those that used photo-activatable fluorophores use relatively large illumination doses and high fluorophore concentrations and the effect on cell physiology should be carefully observed. The illumination dose necessary for imaging a stationary fluorophore has been shown to be reducible by spatially modulating intensity (Hoebe, Van Oven et al. 2007). While the intensity of light striking the edge of the observed fluorophore is retained, the light intensity striking both the centre of the observed fluorophore (where the SNR is high) and the dark background (where the SNR will never be high) is reduced. Even with standard non-photo-activatable fluorescence microscopy there is an issue of phototoxic 
free-radical generation, which ultimately limits the maximum duration of observation of a living sample. However, this effect is strongly wavelength dependent, getting worse at shorter wavelengths. To a certain extent this is being alleviated by the application of fluorescent probes than are excited towards the red end of the visible light spectrum or even into the near infrared.

In living cell samples, one must consider carefully the strategy for introduction of the labels. In order to bypass the cell envelope, a variety of strategies are available including microinjection and protein transduction (Tinnefeld and Sauer 2005), though such methods work poorly for bacterial cells which are commonly used as model experimental organisms. A more universally applicable and elegant approach uses modified cell strains in which several key proteins can be expressed as FP-fusion constructs. The genomic encoding approach has been shown to produce fluorescentlytagged protein at levels which are comparable to the unmodified cell strains (Leake, Chandler et al. 2006). This contrasts with the more common approach of plasmid expression that would produce significantly more protein than would occur naturally. Due to the size of the FP tag being comparable to that of the protein under study, there may be impairment of functionality.

Careful selection of the FP is important (Shaner, Steinbach et al. 2005). The FP should express efficiently and without toxicity to the system under investigation (Gerlich, Beaudouin et al. 2001). It should be bright enough to be distinguishable from the background autofluorescence and should be sufficiently photostable to emit for the required excitation exposure. When two or more types of molecules are labelled there should be minimal cross-talk in the excitation and emission spectra of the FPs used (this 
is not the case in FRET investigations in which case we wish to maximize donor-acceptor FP spectral overlap).

Ultimately measurement is often made in a low SNR regime, which sets a limitation onto the extent of processes which can be investigated simultaneously. The brightness of an FP recorded by the microscope's image sensor is dependent on a number of variables. These include the intrinsic FP brightness, the optical properties of the imaging system (excitation wavelength and intensity, and the transmission spectra of components such as filters and dichroic mirrors used), and ultimately the sensitivity (dependent on wavelength) of the CCD itself. At a basic level this requires that the spatial and temporal resolution be high enough. This means that one must carefully consider the balance between sensitivity of detection, speed of acquisition, and viability of specimen (Stephens and Allan 2003).

Fluorescence microscopy in live-cells has been used to observe gene expression and transcription, translation and replication dynamics in real-time (Xie, Choi et al. 2008). Protein molecules have been shown to be produced in "bursts" with copy numbers following a geometric distribution ( $\mathrm{Yu}, \mathrm{Xiao}$ et al. 2006). The regulation of gene expression was investigated by observation of transcription factor binding to DNA (Elf, Li et al. 2007). It was possible to infer the proportion of time a repressor protein (in the well-studied "lac operon" model bacterial system of gene expression) spends diffusing along and non-specifically bound to DNA. Methods have been developed to fluorescently label RNA, previously elusive due to its high mobility and lack of longterm stability in the living cell. Results showed RNA to be non-uniformly distributed 
within the cell and to be localized in apparently ordered helical structures along the cell axis (Valencia-Burton, Shah et al. 2009).

\subsubsection{The Detection OF Single-Molecules}

The structure of this review is based on the premise that the primary interest is in the underlying set of scientific questions, and the methods we devise to address these are necessary by-products to addressing such questions, as opposed to being an end in themselves. In this case, a fresh approach may be to consider the type of information one wants, and then appraise a broad range of experimental techniques that could conceivably be used (even if in reality certain details preclude use of a subset of the conceivable methods). So a series of general questions are asked relating to the capabilities of SME, and an attempt is made to answer each with a varied range of specific examples of successes from the literature (Figure 3). The questions are roughly ordered in increasing levels of sophistication as to the type of information obtained or manipulation achieved. 


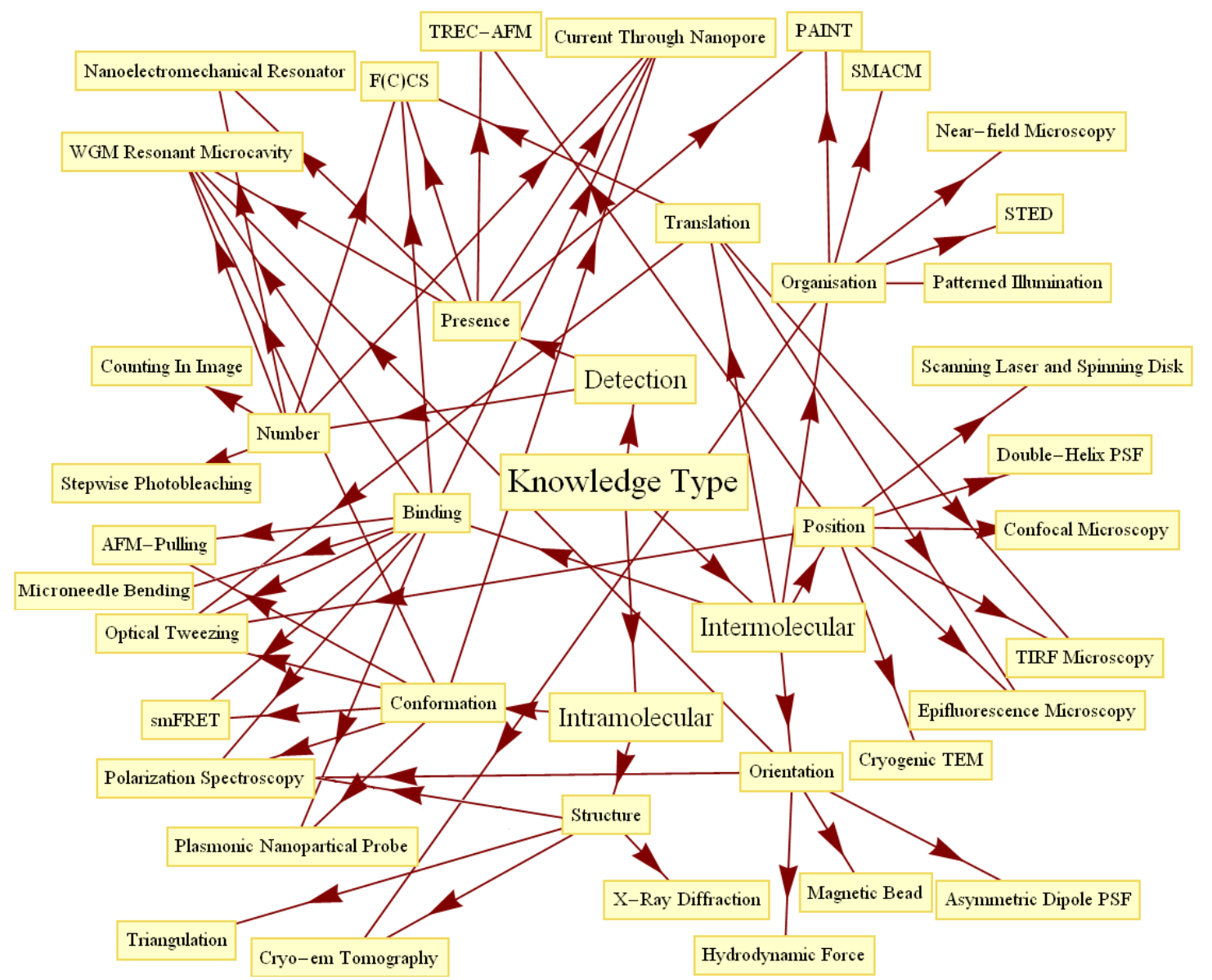

Figure 3 A capability-oriented network schematic of single-molecule experimentation.

\subsubsection{Is a Single-Molecule Present (or Absent)?}

The presence/absence of a single type of molecule may have a pronounced effect on the system of which it is a part. For example, an inhibiting effect on a chemical pathway, an abnormality in a gene knock-out mouse, or more simply a characteristic colour. But in these cases, there is no knowledge or control of the number of those molecules, no direct quantitative measure on a single molecule. Observation of such effects would not be classed as single-molecule observations. 
Biomolecular mechanisms may indicate the presence of a single molecule. The presence of a single molecule (e.g. ATP) could conceivably be evidenced by the energy it transfers to a molecular motor, though one single processive step may be impossible attribute above the thermal noise. The presence of a molecule might be inferable from the effects on the conformation of a second molecule such as a membrane pore, pump or receptor. If the single molecule gates an ion-channel, it may have an effect (that is generally stochastic (Colquhoun and Hawkes 1981)) on membrane resistance. The validity of using this mechanism as a "single-molecule assay" would depend on whether it is a specific type of single-molecule that activates the membrane pore. Presence could be inferred if the molecule would open/close the pore for a long enough time for an observable and significant conductance change. The first measurements of single ionchannel conductance changes were achieved via patch clamping in 1976 (Neher and Sakmann 1976). Ion-channels may be gated by voltage, $\mathrm{pH}$ and the presence of ligand molecules (Millar 2001) and recently have been integrated in modern bio-electronic devices (Misra, Martinez et al. 2009).

A charged single molecule might be registered through direct contact with a detector electrode. In this case the detector would require high sensitivity and an absence of noise, requiring highly controlled environmental conditions, and the likely cooling of the electronics in order to reduce dark current.

If all the molecules of a sample are spatially constrained in a sparse monolayer on a surface, an image can be constructed. A technique with molecular resolution will make evident the presence of a molecule and might enable some form of identification. Popular microscopic techniques capable of resolving molecules include transmission 
(TEM) and scanning electron microscopy (SEM) and the scanning probe methods of near field optical microscopy, scanning tunnelling microscopy and AFM. The applicability of these methods depends further on the properties of the molecules and the environment.

Simultaneous topography and recognition imaging (TREC) uses an AFM with a cantilever tip carrying a ligand molecule (Stroh, Wang et al. 2004). Through specific binding to molecules of interest, and the associated decrease in cantilever oscillation amplitude, simultaneous measurement of topography and molecular recognition is achieved (Preiner, Ebner et al. 2009).

More technical capabilities become available with molecule-specific spatial constraints. For this, an attachment/confinement mechanism is required, though it does not necessitate labelling the molecule with an observable molecule or nanoparticle. If a molecule can be probed without the conjugation of a label, several potential problems may be avoided. An adequately specific label may be hard to find, and conjugation may be difficult. Once attached, it may sterically hinder the molecule of interest and interfere with its conformation.

Through some form of attractive or confining mechanism, a single-molecule in solution may be immobilized at a known position such as on a latent pattern on a substrate surface, or the surface of a probe. Recent work used sequential adsorption of protein A and IgG to a silver nanoparticle micropatterned surface (Combs, Chang et al. 2011). Such high ordering of a pattern can enable the separation of a SERS-enhanced spectra (note the review (Pieczonka and Aroca 2008)) of the biomolecules of interest, from that of the substrate and non-specifically adsorbed species. This approach allowed detection of biomolecules down to almost $500 \mathrm{fM}$ concentrations. 
Silicon nanowire arrays have been used to detect DNA molecules at a concentration limit of 10fM (Gao, Agarwal et al. 2007). Here, the nanowires were functionalized with peptide and nucleic acid, and resistance changes were measured upon hybridization with the DNA. More recently, single-molecule detection of DNA hybridization was achieved with a carbon nanotube field effect transistor (Sorgenfrei, Chiu et al. 2011). Conductance changes were measured when a diffusing strand of DNA hybridized with a complementary strand that was covalently bound to a point defect in a carbon nanotube.

Two ensemble optical techniques are used to register the addition of molecules to a surface through interaction with an evanescent field. Dual polarization interferometry (DPI) works by virtue of the change in polarization of the field, and the Surface plasmon resonance sensor (SPR) works by virtue of resonance shift of electrons at the interface between the surface and a thin metal layer. In 2007, SPR was advanced to reach singlemolecule sensitivity through the incorporation of a "whispering gallery" microcavity (Armani, Kulkarni et al. 2007). This form of sensor has been shown to have potential for a multitude of further capabilities (Vollmer and Arnold 2008).

In the simplest case, a detected molecule may be identified through knowledge that it is of the only species present. A number of ensemble techniques spatially separate the molecular species in a sample then make measures of respective abundance. Separation has been achieved through a range of mechanisms by virtue of molecular mass, size, and shape. Molecules may be separated into sedimentation bands within a density gradient in an analytical centrifuge. In dialysis, the solvated sample is filtered through membranes of set pore sizes. In the chromatography methods, the sample is 
introduced to a medium in which there is a stationary and a transient phase and molecules are separated based on their flow rate. In conventional mass spectrometry, an ionization process creates fragments of molecules with different mass-to-charge ratios. The fragments are then separated through the differential effect on their trajectories as they pass through a magnetic field.

Alternative forms of mass spectrometry have been approaching single-molecule sensitivity. The vibrational frequency of a nanoelectromechanical resonator is exquisitely sensitive to small variations in mass. This has been made use of in a novel form of mass spectrometer, to infer the adsorption of molecules in real-time (Naik, Hanay et al. 2009). The approach is theoretically capable of mass sensitivity below one Dalton. Another paradigm for mass spectrometry was to use a single membrane pore and observe the effect on electrical conductance (Robertson, Rodrigues et al. 2007). The residence time of a molecule in a pore may increase monotonically with its mass and have a measurable effect on ionic current.

\subsubsection{How Many Single-Molecules are there?}

The ability to robustly quantify how many biological molecules are involved in any given process, for example how many sub-units really constitute a functional complex in a molecular machine, is vital to the understanding of the real molecular mechanisms involved in living soft condensed matter. Single-molecule methods excel in determining molecular-level concentrations, particularly when the sample is too small for an ensemble method as in the case of a cell where the copy numbers of many proteins are 
very low. In many cases, the number of pathogen molecules necessary to establish disease is very low, and there has been much work to develop techniques capable of measuring smaller and smaller concentrations as a result.

Counting can be seen as an extension of registering the presence of a molecule type. At some level, there must be a reasonable confidence that it is counting and not kinetics, for example, being able to distinguish between repeat signals from the same molecule over time. If measurements are made simultaneously there must be some way to distinguish the separate signals from the different molecules. Measurements made over extended periods of time require some mechanism that rules out a molecule from repeat measurement (e.g. photobleaching).

For simultaneous counting, imaging is an obvious choice, though molecular motion during the capture period must be ruled out. This could be done by fixing all molecules to a surface, though this may take the system far from its native physiological state. If the image is analysed as a single channel recording (as AFM or SEM micrographs, when interpreted as purely topographical depictions), the resolution must be high enough for the number density of molecules. As described in section 1.1.4.3, fluorescence imaging 'Super-resolution' techniques exist that lower the observed molecular number density (through modulation the fluorescence emission) in order that the spatial resolution is adequate to distinguish individual molecules. If the molecules are large enough to be visualized no labelling is required. For type-specific counting, molecules may be identified by the knowledge that they are the only species present, or based on their form. 
In the established ensemble method of spectrophotometry, the transmission of light through a sample is measured as a function of wavelength from which the concentration can be inferred. Mean reactions from populations of several thousands of molecules can be observed also, where dyes are present whose fluorescent activity changes upon binding to specific chemicals.

With the use of a fluorescent label, measurements of sub fM concentrations have been achieved (Rissin, Kan et al. 2010). Low abundance proteins were captured with antibodies on microscopic beads (with low likely hood of more than one protein per bead according to Poisson statistics) and labelled with an enzymatic reporter capable of producing a fluorescent product. The beads were then isolated in arrays of femtolitresized wells, into which no more than one could fit. Because of the resulting confinement, a high fluorescent signal was observable from those wells in which the proteins of interest were present. The percentage of wells showing fluorescence was then taken to give a measure of concentration. This approach enabled protein concentrations of ca. $10^{-19} \mathrm{M}$ to be measured.

In fluorescence correlation spectroscopy (FCS), analysis is made of fluctuations in the fluorescence intensity from diffusing labelled molecules within a confocal volume (Haustein and Schwille 2007). From this it is possible to determine the localized concentration and molecular mobility. Any change that affects a molecule's mobility may be picked up by FCS. With fluorescence cross-correlation spectroscopy (FCCS), one can determine common features in signals from two distinct fluorescence channels. This may enable the proportion of particles that are double-labelled (i.e. bound) to be determined. The fluctuations observed in FCS are more pronounced, the lower the mean 
number of molecules. This means that the upper bound of concentration is limited by the size of confocal volume and accordingly attempts have been made to produce smaller observation volumes. Two-dimensional arrays of zero-mode waveguides, essentially small holes in a metal film deposited on a microscope coverslip, have been used to this effect. Beyond a cut-off wavelength no propagating wavelength modes may exist and illumination is evanescent from the base of the waveguide. This has the effect of delimiting to sub-wavelength all three spatial dimensions of the excitation volumes (Levene, Korlach et al. 2003).

Counting of molecules in cells brings new challenges. Stochasticity exists in the expression of constituents resulting from intrinsic and extrinsic noise (Elowitz, Levine et al. 2002). One approach is to lyse the cell and analyse the constituents in vitro, possibly using a method described above. This will fail to pick up the heterogeneities in the spatial distribution or molecules in an active cell, but may at least allow for a full accounting of raw molecule numbers to be carried out. Micro- and nano-fluidic "lab-ona-chip" devices (Huang, Wu et al. 2007) can potentially combine the processes of manipulation, lysing, fluorescent-antibody labelling, separation, and protein quantification for a single cell. The single-molecule pull-down approach may enable isolation of complete protein complexes in vitro for examination through fluorescence methods (Jain, Liu et al. 2011).

Ideally a non-perturbative approach with high time resolution would count molecules in regions of living cells. The phenomenon of photobleaching has been taken advantage of in order to count the number of molecules within an active membrane bound complex in a living cell (Leake, Chandler et al. 2006). Using total internal 
reflection fluorescence (TIRF) microscopy a video was taken of GFP-labelled proteins (in vivo) involved in force generation in the bacterial flagellar motor. Analyses were made of region of interest encompassing immobilized flagellar motors. Intensity was measured with time and exponential photobleach decays were observed with step-like behaviour visible near the tail of the measured intensity versus time traces. The underlying periodicity in the intensity was examined in a power spectrum of the pair-wise difference distribution of each bleaching curve. A fundamental peak was then identified as a possible indicator of the intensity a single fluorescent protein. Dividing the initial total intensity of the region of interest by this value gave an estimate of the number of fluorescent proteins present in the motor.

Recently a yellow fluorescent protein fusion library of $E$. coli was generated with 1,018 different proteins labelled for the first system-wide analyses of expression (Taniguchi, Choi et al. 2010). A microfluidic chip was used with fluorescence imaging of 96 strains in parallel, to quantify the protein and respective mRNA presence with single-molecule sensitivity. Investigating Intramolecular Characteristics

\subsubsection{What is the Structure of a Single-Molecule?}

Biomolecules typically have many levels of organisation that result from environmental conditions and the sequence of constituent atomic groups. The form of a protein is generally that which minimises free energy and results from the order of its amino acids. Numerous ensemble techniques have been applied to determine molecular structures. Circular dichroism (CD), the differential absorption of left and right circularly 
polarized light, has been used to indicate the presence of chirality and relative proportions of different molecular structures. Such investigation has achieved single-molecule sensitivity (Hassey, Swain et al. 2006) revealing strong intrinsic CD responses and indication of molecular orientation. In the presence of an external magnetic field, magnetic nuclei will resonate between spin states upon absorbing electromagnetic radiation of a frequency that is dependent on their local chemical environment. Through nuclear magnetic resonance (NMR) spectroscopy, it is possible to obtain separate signals from each atom in a protein that can be characterized by frequencies that provide both distance and orientation constraints for structure determination (Opella and Marassi 2004).

Diffraction investigations such as x-ray, neutron and electron diffraction of crystalline samples have revealed the atomic structures of many biomolecules. There however exist many species that have not been successfully crystallized. The ordered ensemble of molecules present as a crystal has been necessary to gain enough signal before the sample is destroyed by the impinging beam. However, theoretically it was believed that if a pulse of $\mathrm{x}$-rays was intense enough, it could generate adequate signal, from a single-molecule, before the molecule exploded due to heating. It was recently shown that it should be possible when a purpose built x-ray laser (the most powerful in the world) was shone into a Mimivirus to achieve a reconstruction with a ca. $32 \mathrm{~nm}$ spatial resolution (Seibert, Ekeberg et al. 2011). With higher irradiance pulses and detectors with higher dynamic range, it is foreseen that the structures of single-molecules may be produced by such an approach. 
With electron microscopy structural features in fixed biological samples may be visualised. A contrast agent is added to the sample that is associates preferentially in different parts of the sample and structure is revealed as electrons are variably transmitted (TEM) or scattered (SEM). In cryogenic electron microscopy (cryo-EM), the rapid freezing to cryogenic temperatures can allow biological samples to survive the vacuum conditions necessary for TEM without the need for fixing. Standard cryo-EM with single particle reconstruction (where many similar particles are imaged and correlated) has been able to produce $3 \mathrm{D}$ data of a high enough resolution to be fit with an atomic model (Zhang, Settembre et al. 2008). With cryo-EM tomography only one particle is examined, and 3D structure reconstruction is produced from images at a series of orientations around its centre (Downing, Sui et al. 2007). A strength of cryo-EM is the capability to generate maps of the relative locations of many proteins in a complex. Higher resolution molecular structures generated from crystallographic methods may be used to interpret the tomographs with less ambiguity.

The basis for a single-molecule approach to determining protein structure has been developed that makes use of mechanical triangulation (Dietz and Rief 2006). With prior knowledge of the protein sequence, Angstrom-precise respective positions of three amino acids in GFP were found in solution. The process involved measuring the change in distance between each pair of the three acids before and after pulling them apart to a completely stretched conformation. Several approaches are now being explored into taking advantage of the constricted geometry of pores to infer the bases of a DNA molecule (Branton, Deamer et al. 2008). Other single-molecule sequencing approaches are being developed including "sequencing by synthesis" and with TEM (Gupta 2008). 


\subsubsection{Can we Determine the Conformational State of a Single-Molecule?}

As well as being affected by the incessant molecular collisions due to thermal motion, the conformation of biomolecules develops with folding from the initial state of synthesis, and may change further upon binding (in allosteric molecules). The functionality of biomolecules is largely based on their 3D form and the energy landscape that guides conformational change (Zhuang and Rief 2003).

Intramolecular distance can be measured by using two different coloured fluorophores attached to different sites on a molecule. A two-colour version of FIONA, single-molecule high-resolution colocalisation (SHREC), was developed capable of interfluorophore measurements below $10 \mathrm{~nm}$ in resolution (Churchman, $\tilde{\mathrm{A}}-\mathrm{kten}$ et al. 2005). The molecule of interest is double-labelled with two chromatically different fluorophores and excited with lasers corresponding to the excitation spectrum of each. The emission is separated with dichroic mirrors and filters and directed to differing positions on a CCD. A mapping function is then used to correlate the images and determine the interfluorophore distance.

For high precision measurements of inter-fluorophore distances of up to ca. $10 \mathrm{~nm}$, FRET is employed. FRET is made use of in two main configurations, the most relevant to conformational measurements being where one molecule is labelled with the donor and acceptor (Hohlbein, Joyce et al. 2011). The second configuration is where one of two molecules that may associate is labelled with a donor, and the other an acceptor; this is used to observe binding. Configurations that involve more than two fluorophores per molecule are also being developed (Roy, Hohng et al. 2008). Using alternating-laser excitation (ALEX) of single-molecules it is possible to characterise both intramolecular 
distances (based on donor, acceptor FRET) and to infer whether a molecule is labelled with a donor, acceptor or both to give a measure of stoichiometry. This can be used in the spectroscopy of molecules diffusing through a confocal volume, or in microscopy of immobilized molecules on a surface (Kapanidis, Laurence et al. 2005).

Denaturation, the disruption of the non-covalent intramolecular bonds, of molecules in an ensemble can be achieved with solvents or high temperatures. At a single-molecule level conformational changes may be induced and observed through methods of force spectroscopy.

Techniques that probe with force include optical tweezing, magnetic tweezing, AFM, glass microneedle manipulation and flow-induced stretching. Force based methods enable manipulation over six orders of magnitude in length (10-10-10-4 m) and force $\left(10^{-14}-10^{-8} \mathrm{~N}\right)$ (Neuman and Nagy 2008), though typically the scales relevant to biomolecules are $\mathrm{nm}$ and $\mathrm{pN}$. Direct mechanical measurement of elastic properties of single-molecules can be made, for example on DNA (Smith, Finzi et al. 1992), and to observe the metastable and transient folding states on large molecules such as GFP (Dietz and Rief 2004). Through combining optical tweezing and FRET improvements in force sensitivity have been achieved (Hohng, Zhou et al. 2007).

A complementary approach to force-based SME is that of steered molecular dynamics. This is where in silico molecules are probed as in vitro experiments, with the aim of gaining deeper insight into the mechanical mechanisms at play (Sotomayor and Schulten 2007). Coarser modelling approaches have been developed for understanding features such as anisotropic deformation response of molecules over times scales closer to those of force-based experiments (Dietz and Rief 2008). 


\subsubsection{INVESTIGATING INTERMOLECULAR DETAILS}

\subsubsection{The Orientation of a Single Molecule}

Linear dichroism is an ensemble polarized spectroscopy technique that can provide information about relative orientations of sub-units of biomaterials and orientations of the whole biomaterial with respect to an orientation axis (Hicks, Kowalski et al. 2010). The orientation of molecules in a cuvette affects the degree of absorbance of light linearly polarized parallel and perpendicular to an orientation axis. Polarization spectroscopy of single fluorophores has been used to probe rotation of surface immobilized molecules and the polarization anisotropy of freely diffusing molecules (Ha, Laurence et al. 1999). Recently, single-molecule fluorescence polarization made evident continuous helical rotation involved in biofilament elongation and depolymerisation (Mizuno, Higashida et al. 2011). Fluorophore dipole orientation can also be determined from a real space image through maximum-likelihood fitting of an anisotropic diffraction limited spot (Mortensen, Churchman et al. 2010).

It is often useful to control the orientation of a molecule. In ensemble linear dichroism, elongated molecules may be co-oriented by shear forces produced by rotation of a cylinder within the measurement cuvette. Standard methods of single-molecule orientation include tethering a molecule to a surface at one end and exerting a hydrodynamic force, or magnetic force on a magnetic particle attached to the other end (Smith, Finzi et al. 1992). Torque may be applied directly to the magnetic particle, as is the case in experiments on the super-coiling of DNA (Strick, Allemand et al. 1996). 


\subsubsection{Single-Molecule Spatial Location}

The location of a single-molecule could conceivably be determined in several ways. Triangulation could be based on a quantity (such as magnitude of irradiance) that monotonically changes with distance from the molecule. The molecule could be located spectroscopically by the effect of the environment, if for example fluorescence emission changes in a way that has been pre-mapped. Likewise, the response may indicate location with illumination or probing that varies with position in some manner.

The standard approach is to create a map of the area or volume, and in it the molecule is evident. This can be done with a scanning method or by a far-field method. If the molecule is constrained to a known geometry such as a flat surface or a bacterial membrane, less information may be necessary to infer its location. Reducing the regions of probing or illumination is a design principle across a number of SME techniques including laser scanning confocal microscopy (LSM or LSCM), scanning near-field optical microscopy (SNOM/NSOM) and TIRF microscopy. In SNOM/NSOM light is sent through a sub-wavelength optical aperture in a transparent metal coated probe tip that scans sample topography. This produces illumination of a surface region that is laterally smaller than that in confocal excitation (Hecht, Sick et al. 2000).

There is a commonly employed method that gives typically a ca. 10-fold improvement in the precision with which one can locate an isolated object whose characteristic length dimensions are less than the diffraction-limited optical resolution of a far-field imaging system. The method, applied in localization and tracking contexts, 
involves calculation of the centroid of the two-dimensional Gaussian function of the spatial distribution of fluorescence intensity from the object, which is fitted over the object in the image. The precision of the calculated centroid is dependent on the number of photons emanating from the fluorophore (Thompson 2002). Noise affecting the precision comes from the fluorophore (in the form of "Shot noise"), and the background (a result of factors including out of focus fluorescence, and readout noise of the CCD), as well as from uncertainty of where precisely a detected photon was incident within a given CCD camera pixel (so-called pixelation noise).

TIRF microscopy is an approach that allows for the excitation of a broad but very shallow region of space beyond a cover slip. A beam emerging from the edge of the objective lens, passing through oil and entering the cover slip, strikes the sample surface of the slip at an angle that results in total internal reflection. At this point an "evanescent" field is produced with a 1/e penetration depth of typically $\sim 100 \mathrm{~nm}$ (normal to the surface and into the sample) (Tokunaga 1997). This field is useful as it can be used to excite fluorophores within a cell's membrane with minimal excitation in other regions, dramatically reducing the noise due to scattering and fluorescence of molecules in other parts of the cell and of the surrounding buffer. TIRF microscopy has made it possible to discern the presence of, and track, single membrane proteins above the usually prohibitive noise (Mashanov 2003).

TIRF microscopy and the centroid calculation localization process were combined and called fluorescence imaging with one nanometre accuracy (FIONA) (Yildiz, Forkey et al. 2003). The method was famously used to examine the step lengths of myosin molecules (labelled with single $\mathrm{Cy} 3$ dye molecules) moving along actin filaments in 
vitro. Analysis of the processive step lengths was seen to indicate the relevance of a particular "hand-over-hand" model for myosin motion.

Alternative approaches use additional patterned illumination of the sample to affect fluorescent yield and obtain higher resolution. One technique inhibits fluorescence in the outer regions of the illumination point spread function through stimulated emission (STED) (Hell and Wichmann 1994). In saturated pattern excitation microscopy (SPEM) higher resolution is obtained from the generation of higher spatial harmonics in the emission pattern. These result from the nonlinear dependence of fluorescence on illumination intensity when fluorophores are saturated with standing wave illumination (Heintzmann, Jovin et al. 2002). Recent work has massively parallelised the STED technique to allow much faster imaging of large fields of view (Rowlands, Yew et al. 2013).

The point spread function (PSF) of a standard microscope gives little information on the axial position of a fluorophore and in diffraction limited system the resolution is typically three times poorer than in its focal plane, though various ingenious optical techniques have been applied to reduce this (Egner and Hell 2005). Techniques including astigmatic and biplane imaging and use of a double-helix point spread function have achieved axial location precision of under $10 \mathrm{~nm}$ (Badieirostami, Lew et al. 2010).

\subsubsection{The Relative Position of Many Single-Molecules}

The location of many fluorophores within a diffraction limited spot ("Superresolution imaging") may be attempted simultaneously or sequentially. Fluorophores may be imaged sequentially through the ability to have different fluorophores fluorescing 
at different times. This was first achieved through the STED technique of inducing fluorescence from sub-diffraction limited regions (Klar, Jakobs et al. 2000).

Photobleaching has been made use of in techniques to improve spatial resolution and reduce background fluorescence, aiding the tracking of molecules. Single-molecule high-resolution imaging with photobleaching (SHRImP) is a technique that takes advantage of the quantal nature of photobleaching to resolve two fluorophores separated by distances down to $5 \mathrm{~nm}$ (Gordon, Ha et al. 2004). A movie is made of two fluorophores moving in close proximity to each other. The total intensity is recorded until one molecule photobleaches and a stepwise intensity decrease is observed. The remaining fluorescence is said to come from the one remaining fluorophore and a twodimensional Gaussian distribution is fitted to its image. This allows the (FIONA) localization of the remaining spot to a precision of $\sim 1.5 \mathrm{~nm}$. The previous frame is then observed in which both fluorophores are photoactive and the single fluorophore Gaussian distribution is subtracted from the total to determine the intensity distribution of the remaining fluorophore. Comparison of their localization allows for the high precision distance measurement. It should be remembered that the distance measured is only the image plane two-dimensional projection of distance.

In some fluorophores a saturatable transition can be induced between two conformational states, by using much lower intensities of light than are necessary for rapid bleaching. This mechanism was first used to switch on and off fluorophores as a basis for super-resolution imaging in the RESOLFT technique (Hofmann, Eggeling et al. 2005). There has been much interest in techniques that use this process to locate densely distributed biomolecules. In photo-activated localization microscopy (PALM) (Manley, 
Gillette et al. 2008) photo-activatable fluorescent proteins are used, all of which are initially in their inactivated state. Then the sample is irradiated for long enough to activate a sparse subset which is imaged until bleaching occurs. This process is repeated until all fluorophores have been activated, imaged and bleached. Summing the frames yields an image with resolution of 2-25 $\mathrm{nm}$.

In sub-diffraction-limit imaging by stochastic optical reconstruction microscopy (STORM) (Rust, Bates et al. 2006), fluorophores are switched between a fluorescent and dead state in a reversible manner by light of different wavelengths. The process involves going through cycles where only a fraction are activated and imaged in order that (as with PALM) the population appears sparser than it is. After a sufficient number of cycles, the images are correlated to map the position of all fluorophores. Originally, organic fluorescent dyes were applied in vitro for STORM investigations (Rust, Bates et al. 2006). Next, the process was extended to multiple colours (Bates, Huang et al. 2007). In 2008, the method was extended into the third spatial dimension giving image plane resolution of 20-30nm and a depth resolution of 60-70 nm (Huang, Jones et al. 2008). High quality super-resolution imaging of molecules in a living cell is now possible with fluorescent proteins (Lee, Thompson et al. 2011).

In a further approach, points accumulation for imaging in nanoscale topography (PAINT), super-resolution images of surface structure are generated from transient binding and associated dequenching of fluorophores diffusing from solution (Sharonov and Hochstrasser 2006).

New labelling approaches may have significant impact on in vivo imaging. The SNAP-tag and CLIP-tag fusion proteins can be labelled specifically with a choice of 
molecular probes in a living cell (Gautier, Juillerat et al. 2008). Developed for use in PALM type imagining investigations, HaloTag-based target-specific labelling enables with the use of bright organic fluorophores as an alternative to fluorescent proteins (Lee, Lord et al. 2010). A fluorescent flavoprotein has also been developed that ultimately enables correlated electron microscopy (Shu, Lev-Ram et al. 2011). Live fluorescent images of the genetically encoded protein may be taken, and then higher resolution TEM images of the fixed cell may reveal finer structural details.

\subsubsection{Single-Molecule Translational Activity}

An extension of localization and imaging is the tracking of molecules over time. This requires that the molecules be repeatedly localized within a time window that is short enough to resolve the dynamics of interest. It is attractive to carry out motility experiments in vitro because one need not consider the effects of high fluorescence backgrounds, complicated filament geometries, non-uniform drag coefficients and other enzyme activity (Toprak and Selvin 2007). One may also modify the environment to regulate molecular activity. In order to resolve in time the hand-over-hand steps of myosin V, the concentration of ATP (the molecular motor fuel) was reduced to slow dynamics (Yildiz, Forkey et al. 2003).

Force-based methods have also been used to monitor the motion of processive

molecular motors. The transcription process in which RNA polymerase moves along DNA was monitored through conjugated beads held in optical tweezers. The length of 
discrete processive steps and force-velocity relations were obtained (Abbondanzieri, Greenleaf et al. 2005).

In living cells, analysis is often aimed at characterising the motion of a molecule to infer whether it was freely diffusing, or had some sort of biased motion indicative of being involved in a biological process such as active transport. Ensemble molecular diffusion can be characterised by mean squared displacement against time interval, where free Brownian motion is inferred from a linear trend and anomalous diffusion otherwise. Single-molecule tracking data may give access not only to the mean, but to the full probability distribution of square displacements (Schutz, Schindler et al. 1997). Statistical tests have now been developed to distinguish between random, confined, and directed modes of motion in single tracks, and have been applied to the interpretation of three-dimensional in vivo mRNA tracks obtained using double-helix PSF microscopy (Thompson, Casolari et al. 2010). Where the mode of diffusion is already known, FCS may be used to extract the diffusion coefficient and other associated parameters.

In live-cell studies, techniques such as fluorescence recovery/loss in photobleaching (FRAP/FLIP) are used that make use of photobleaching to reduce background fluorescence. In FRAP, a region of interest in the surface of a cell is photobleached typically with a high intensity focused laser pulse. Afterwards, migration of photoactive molecules into the region can become evident and one can make inferences of the mobility of molecules in the membrane. FLIP is in many ways the inverse of FRAP, one makes observations of dark molecules entering a region from a nearby photobleached region. One study using these two methods revealed a counterintuitive dynamic turnover of stator units in the E. coli flagellar motor (Leake, 
Chandler et al. 2006). Using modifications of the same techniques, proteins involved in respiration were investigated and appeared to be localized in mobile membrane-bound patches (Lenn, Leake et al. 2008). When the intermolecular distance is less than the optical diffraction limit, super-resolution methods such as those that use photoactivation or switching are used, however such methods cannot currently be applied to simultaneous tracking of all labelled molecules due to the $\sim \sec$ time scale necessary for image generation being too long to resolve the dynamics that are typically of interest. However, such techniques may still be advantageous with a densely distributed population. Multiple sparse resolvable subsets of molecules may be tracked without photobleaching the remainder. The process can be repeated and many more tracks can be obtained than would have been possible otherwise (Manley, Gillette et al. 2008).

\subsubsection{Observing Molecular Binding and Unbinding}

Bonding characteristics can be explored through both mechanical probing, and monitoring of the binding kinetics. The bond strength of two molecules is a function of the free energy upon binding minus the sum of the free energies when unbound. This is directly related to the equilibrium constant of the binding reaction, which itself can be determined independently from the association and dissociation rates within a mixture.

The application of force to a single-molecule can alter the free energy landscape for a reaction, affecting the thermodynamics and kinetics respectively (Tinoco $\mathrm{Jr}$ and Bustamante 2002). The rupture force for an individual covalent bond was first directly measured through tension on a polysaccharide molecule bound end-to-end between a 
surface and an AFM probe (Grandbois, Beyer et al. 1999). Force-extension curves were produced in which rupture events were evident as kinks in the curve, giving rise to characteristic "sawtooth" patterns when several rupture events are involved (Rief, Gautel et al. 1997). Prior to this, the rupture force between two actin filament monomers was measured through the flexing observed in a glass microneedle bound in perpendicular orientation to one end of the filament (Kishino and Yanagida 1988). The tension was applied from a relatively stiff glass micro needle attached in parallel orientation with the other tip of the filament. In the same study the technique was used to measure the ATPdependent force exerted by myosin heads upon actin filaments.

With suitable time resolution, the techniques capable of molecular detection enable studies of kinetics. In an aqueous solution, binding kinetics can be monitored by the difference in appearance of a bound and freely diffusion fluorophore. In such a way ATP turnover was monitored with TIRF microscopy focussed on the binding site of myosin (Funatsu, Harada et al. 1995). FRET may be employed to study binding via two schemes. If the donor and acceptor fluorophore are on separate molecules, acceptor intensity will increase upon binding. The event may alternatively be monitored with both donor and acceptor on one molecule if there is an associated change in its conformation. Microfluidic devices make possible the miniaturisation of flow based assays and rapid combinatorial processing. Mixing and associated FRET measurements can be orchestrated at high rates enabling measurement of very short lived states (Gruebele 2011). Control of the molecular nano-environment and the effect on binding is being explored through the use of membrane protein "nanoreactors" (Bayley, Luchian et al. 2008). 
The position of a molecule may also track kinetic activity, as in DNA synthesis where an increase in length results from addition of a base. Through two-colour TIRF microscopy, the rate of synthesis of flow-stretched DNA was recently monitored while simultaneously detecting polymerase association on the DNA (Loparo, Kulczyk et al. 2011). Many further investigations have been carried out into protein-DNA interactions (Hilario and Kowalczykowski 2010). Within limited sample areas AFM can produce video of topography with high enough spatial and temporal resolution to capture conformational dynamics. This was recently demonstrated in a study of the behaviour of torque-generating $\beta$ subunits in the stator rings of the rotary molecular motor F1-ATPase at a range of physiologically relevant ATP concentrations (Uchihashi, Iino et al. 2011).

\subsubsection{PerspeCtives}

The single-molecule techniques applied to the living component of soft condensed matter have now entered the next generation phase. The first generation was characterized predominantly by in vitro experiments using one single-molecule technique, generally on isolated, purified molecules divorced from their native context of the living cell. The next generation is characterized by far greater experimental complexity; as we have seen, it is now common to apply multiple single-molecule techniques simultaneously in order to extract multiple dimensions of information from any given experiment. In addition, many in vitro experiments now include multiple molecular components to create a controlled biochemical environment much closer to the native context, with such developments going hand-in-hand with improvements in 
microfluidics design and implementation. Finally, it is now feasible, especially using advanced fluorescence microscopy techniques, to monitor soft condensed matter at a single-molecule level in actual living cells in real-time, with improvements in superresolution approaches permitting knowledge of spatial precision at, in principle, sub-nm levels.

The most likely improvements in the near future will be twofold. One will involve improvements in sampling speed: this will necessitate the development of brighter, smaller, more stable fluorescent dye tags, more efficient dye delivery mechanisms into the living cell and improvements in camera speed and sensitivity technology. This may permit much faster (sub-ms) time scales than are available at present, which may ultimately give insight into the transient, unstable switches in molecular structural states that drive some of the most important biological processes in the living cell.

The second level of improvement is likely to involve force spectroscopy on living cells. Currently, single-molecule mechanical investigations are limited either to in vitro experiments or to in vivo involving molecules which are accessible on the surface of cells (for example, protein complexes integrated into the cell membrane). However, all biological processes at some level involve a force-dependence between a molecule and a substrate. It is probable therefore that more research efforts will in future be devoted to optimizing single-molecule techniques which might allow manipulation and measurement of molecule force inside living cells as opposed to just on their surface. 


\subsection{Preliminary Work and Direction of Research}

This chapter includes work that led to definition of the research questions of my D.Phil.

\subsubsection{Preliminary EXPERimentation}

At the start of my D.Phil., I gave time to helping with my group's existing microscope and a preconceived experiment with collaborators. My group made use of a custom-made multiple-laser fluorescence microscope with a broad range of possible modes of illumination. As well as objective type TIRF, epifluorescence and brightfield illumination, it was possible to illuminate with confocal volumes of different sizes. A back-cooled Electron Multiplying Charge Coupled Device (EMCCD) was used which allowed a very high photosensitivity. The optical principles are explained in more detail in section 2.1. A schematic diagram of the setup is given in Figure 4. 


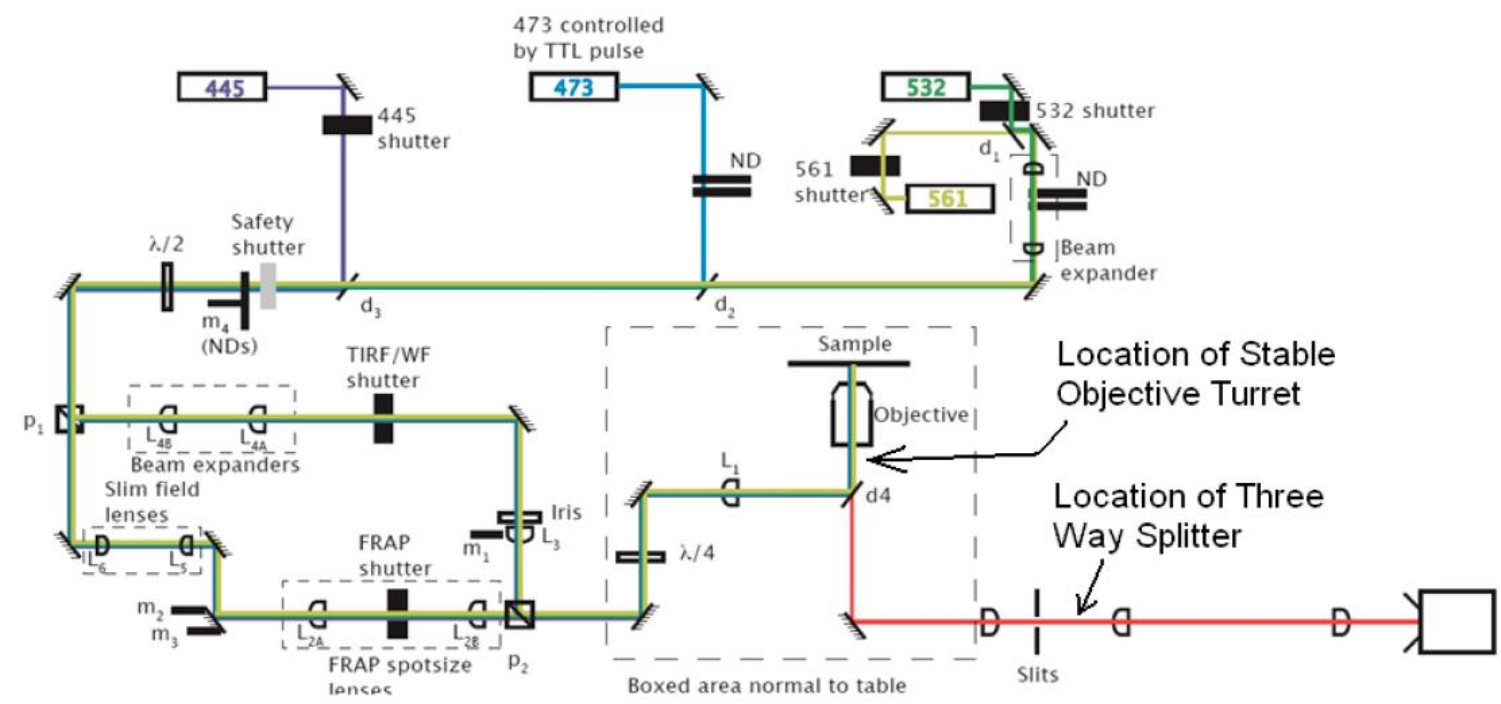

Figure 4 Schematic diagram of the pre-existing fluorescence microscope. The arrows indicate locations of the components I developed for the microscope. Image courtesy of Dr Ian Dobbie.

The first task I took on was to design and build a three-way splitter to isolate the emissions of cyan, yellow and red fluorescent proteins. Figure 5 is a schematic diagram of the optical design. The components are positioned on a small optical breadboard with magnetic mounts so that it can be inserted into and taken out of the microscope many times with no significant change in image positions (Figure 6).

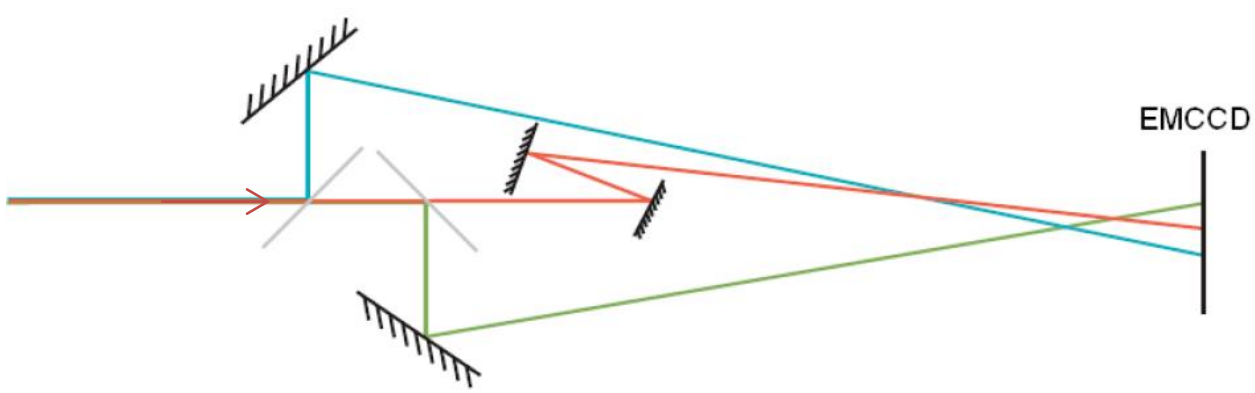

Figure 5 Schematic diagram of the three-way splitter. 


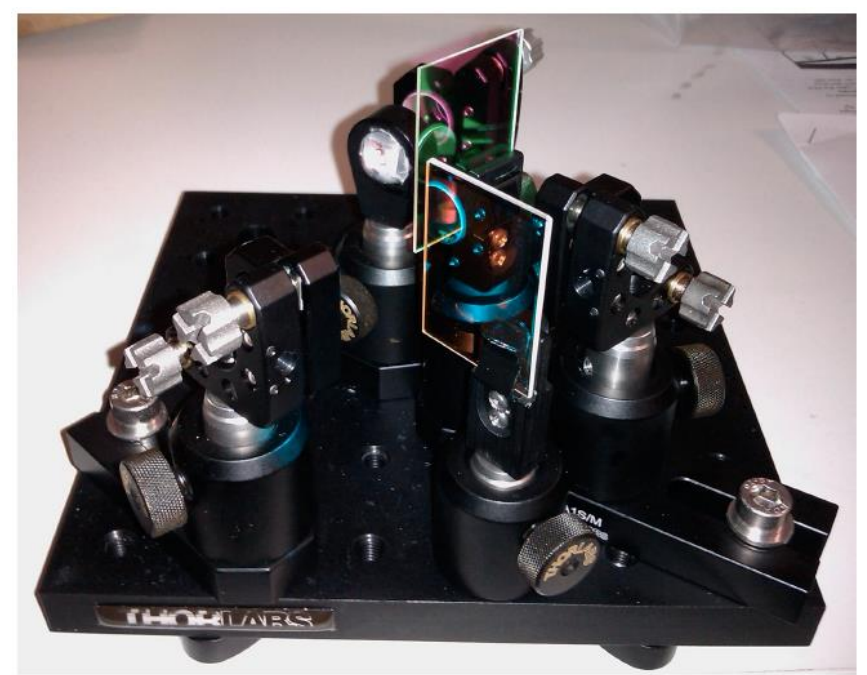

Figure 6 Photograph of the three-way splitter. Blue reflections can be seen from the nearest dichroic mirror and green from the furthest.

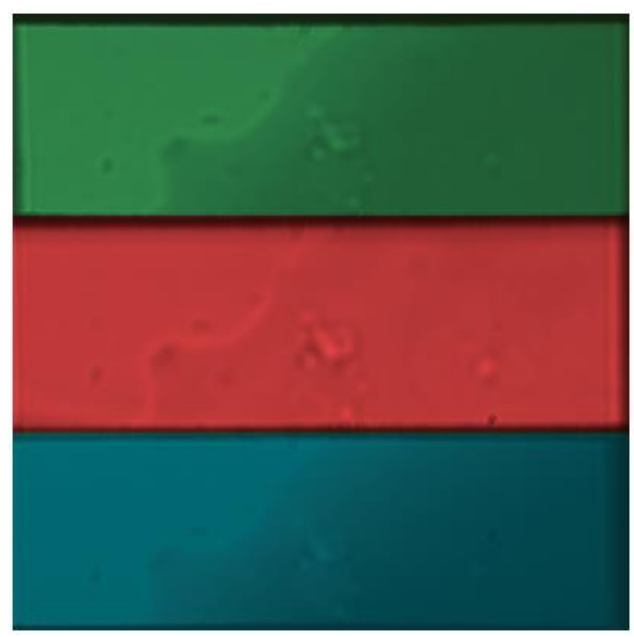

Figure 7 Brightfield transmission image viewed with three-way splitter installed in emission path. The image has been false coloured (the EMCCD is monochromatic) to identify channels.

The objective lens of the microscope was exhibiting sensitivity to minor environmental vibrations (such as those resulting from footsteps). Under-damping of the objective was also evident, resulting in a typical decay time of around two seconds. I 
designed, machined and installed a new objective lens turret (Figure 8). The new turret allows for continued use of the existing focus wheel, but is more rigid and a screw can be tightened to reduce observable vibration when sample focus is found.

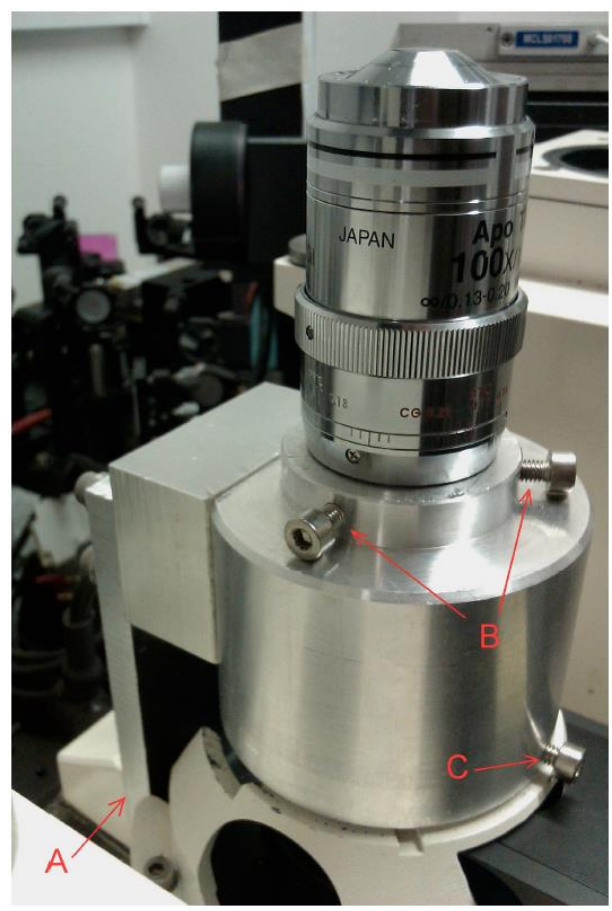

Figure 8 Photograph of objective lens stabilisation turret. A) Interface with wheel-controlled focus mast. B) Screws for securing of objective lens to the turret. C) Screw for locking of turret to microscope base. The original objective turret was solely connected to the microscope base via the focus mast. The new turret was built from less flexible components and given a second point of connection to the microscope base through the screw at $\mathrm{C}$.

Around a month prior to commencing live-cell experiments I began to work on preparation for the experiments. In this period I learned methods of in vitro fluorescence sample preparation, aligned the microscope, improved the efficiency of the filter 
selection, and developed an experimental protocol with fellow group members Professor Mark Christian Leake and Dr Alex Robson.

My collaborators Tchern Lenn (Queen Mary's College London) and Heiko Erdhardt (University of Freiberg) had generated the bacterial strains for investigation. We took several videos $(0.04 \mathrm{~s}$ per image frame) of the strains until photobleaching significantly reduced the SNR. Of each strain we took ten videos with TIRF illumination and ten with TIRF Illumination having previously photobleached roughly half the cell by positioning a FRAP laser pulse over one of the cell's poles. The FRAP pulse was used to lower the fluorescence background in the pole of a cell, resulting in a much higher SNR when a fluorescence complex of interest diffused into the bleached region. Prior to each fluorescence image, a short brightfield video was taken for the purpose of identifying fluorophore locations within the cells and colocalisation-mapping in the analysis stage. We also carried out a number of control experiments including using the same isolated fluorescent proteins in vitro to characterize cross-talk and bleed-through.

After the experimentation phase I gave some input into the redevelopment (by Isabel Llorente Garcia) of MATLAB software for the automated analysis of the fluorescence images. The software was designed to locate and track fluorescent features and to extract information on their mobility, size, stoichiometry, and the colocalisation of features appearing in different channels.

I then played no further role in the investigation and went on to work on related research that was of my own conception (as described in the remainder of this thesis). The analysis and further experiments for this investigation were completed over the course of two years and the results have recently been submitted to the Journal Nature 
Communications. Through the collaboration I benefited by gaining access to bacterial strains for use in my own investigations (see Chapter 5).

\subsubsection{The Challenge of Single-Molecule Live-Cell Fluorescence MICROSCOPY}

The initial experiences gave me a number of insights in to the practical challenges of live-cell single-molecule fluorescence microscopy. The benefit of TIRF microscopy is that it enables significantly higher imaging contrast due to the preferential excitation of the cell membrane over the cell cytoplasm. This reduces the contribution of fluorescence not associated with the proteins of biological interest (Figure 9).

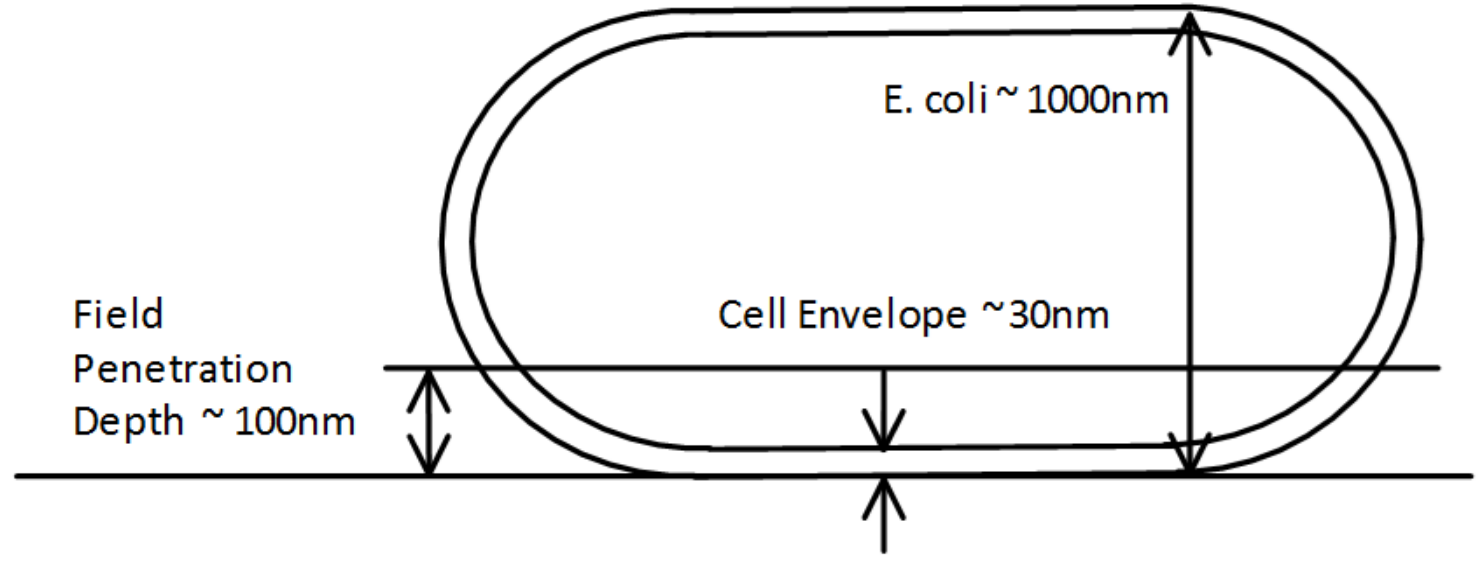

Figure 9 A schematic showing the relevant dimensions of the cell and the extent of the evanescent field. Not to scale.

Using multiple colours allows interesting questions to be addressed such as whether functionally linked molecules are colocalised in the cell (Figure 10). 


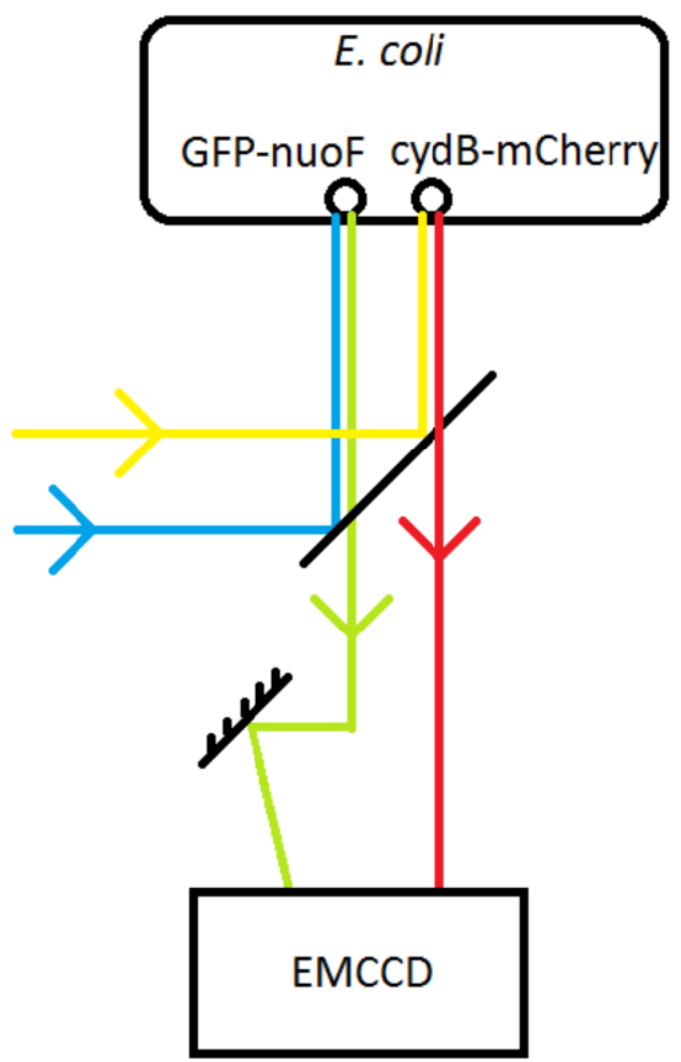

Figure 10 A simplified schematic of a two-colour fluorescence microscopy experiment.

Even with a well-suited technique, achieving intelligible data can be very challenging and a lengthy process of experimental parameter optimisation is typically necessary. Effort is made to balance several parameters in order to reach multiple system-level objectives. As a reduced example, one might want to capture the dynamics of a biological process lasting in the order of seconds in which changes in energy/conformational state might occur in the order of tens of milliseconds. One would need to find adequate laser intensities to balance high time resolution (requiring high excitation intensities for adequate SNR) with low photobleaching rate (requiring low excitation intensities in order that the video may last the entire duration of the process). 
It is also important to consider camera settings such as the use of binning (Swift and Trinkle-Mulcahy 2012). As a specific example, two frames (with false-colouration) from a video of the preliminary investigation are presented in Figure 11.

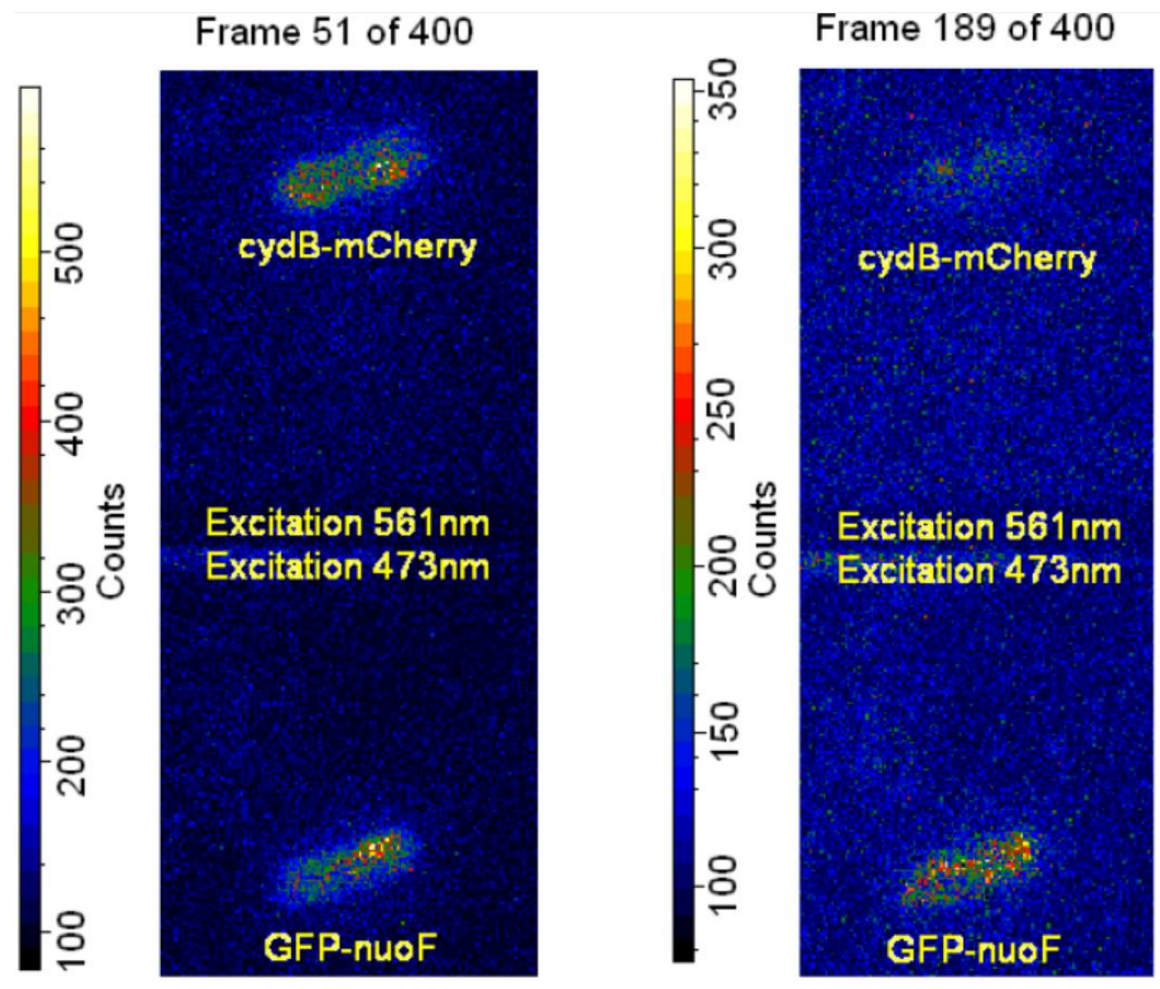

Figure 11 This figure shows two (false coloured) frames of a video of a double labelled strain. Through colour splitting in the emission path one can see the CydBmCherry and GFP-NuoF emission above and below respectively. The lower contrast in the latter image is indicative of the photobleaching that occurred as a result of the sample illumination.

The imaged bacterium was of the cydB-mCherry/GFP-nuoF strain. The fluorescence can be seen to be heterogeneously distributed at both times and in both colours. The colocalisation of $\mathrm{CydB}$ and NuoF is inferred when the cells of a particular frame are overlaid. In frame 51 it appears that the two proteins are located in different 
regions of the membrane. Conversely in frame 189, there appears to be colocalisation of the CydB with the NuoF in the top left region of the membrane.

Though the fluorescence evident in these individual frames does not appear to be distributed in clearly identifiable regions, it should be noted that in watching the entire video it is easier to discern moving circular regions of florescence. These are seen more clearly in the single-labelled strains. As apparent in Figure 11, there may be varying degrees of colocalisation with time. Statistical analysis will need to be carried out to determine whether any observed colocalisation is significant and for future inference of any models of interaction.

\subsubsection{Vision of A System-Level Approach to Single-Molecule Live-Cell FLUORESCENCE MiCROSCOPY}

While it is simpler to consider a reduced model of the experimental system when changing a parameter, data quality is a function of the system as a whole. In live-cell fluorescence microscopy, many key relationships are non-linear, with dependencies that are challenging to reliably envisage and qualitatively combine. Though the instrument can effectively be used as a deterministic system, the interrogation of a living cell means one must be mindful of stochasticity and history dependence.

The view was taken that by engaging with an experiment as a whole system, one may be able to achieve improved experimental performance. Some tools would need to be developed to extend the memory and processing power of the human mind and enable

quantitative and unified calculations. Through analysis of the system structure when 
described in an internally consistent model, the complexity may become intelligible and more manageable. If successful, the use of system level techniques and tools could be extended to other experimental contexts.

Taking a system-level approach requires artificial boundaries to be drawn to define what constitutes the experimental system. The aspects of the system that are described must be chosen based on their relevance to the experimental purpose. Both physical and informational flows exist within the system. A conceptual schematic of the experimental system is presented below in Figure 12.

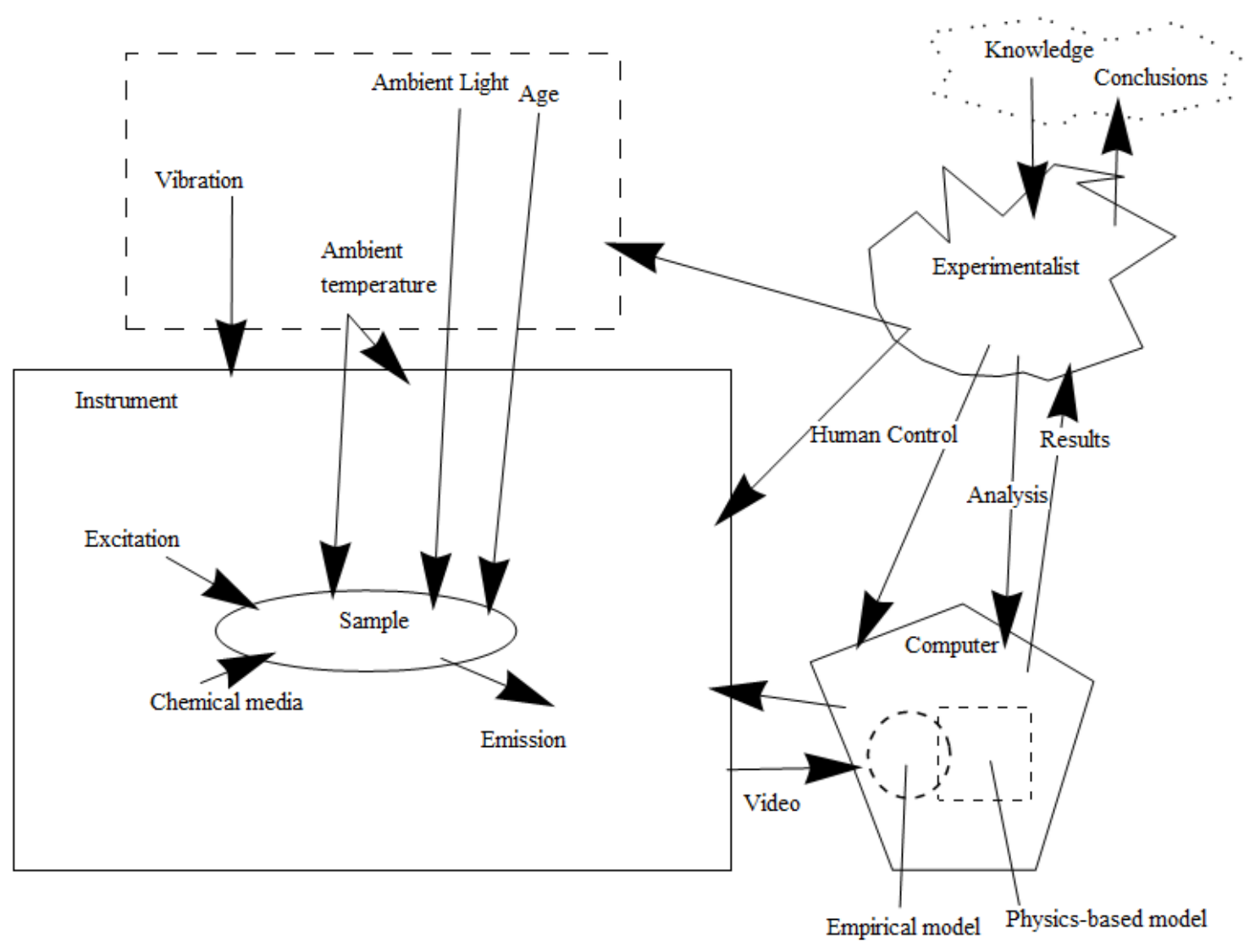

Figure 12 A system-level view of fluorescence microscopy. 
Two complementary D.Phil. research questions were conceived of and pursued:

a) Could a more suitable instrument be made given a system-level view of the research? The most direct and frequently taken route to building of an experiment is to build it for one specific measurement or set of associated measurements. In this D.Phil., the opportunity was taken to explore another approach to the development of a bespoke scientific instrument. There are concepts more typically seen as pertinent in the field of engineering that, when taking a system-level view, are also of relevance to the outcome of a scientific investigation. Such concepts include flexibility, usability, maintainability, cost, and compactness. It would be explored how an instrument could be built that enabled multiple experiment types by multiple different users with minimal reconfiguration and re-alignment.

b) How can available information be made use of quantitatively and in parallel to improve performance of an experiment? The knowledge produced from an experiment is critically dependent on the quality of obtained data. The challenge of experimental complexity is traditionally dealt with in a manual, iterative process. The experimental system would be computationally modelled as a whole, to explore how experimentation could be informed and data optimised through simulation. 
2 CREATION OF THE MICROSCOPY SYSTEM 


\subsection{Development of the Bespoke Microscope}

\subsubsection{OPTICAL MICROSCOPY}

\subsubsection{The Compound Optical Microscope}

The modern compound microscope essentially combines three technologies: Köhler illumination, an infinity corrected objective and tube lens pair, and an electronic image sensor. In the brightfield imaging mode, a sample is probed with visible electromagnetic radiation whereas in fluorescence microscopy the source of imaging photons is the sample itself. Magnified imaging is achieved via the refraction of lenses and the image location and magnification can be calculated through ray tracing. To explain image formation and the limits of attainable resolution it is necessary to consider the diffraction and interference of light.

Köhler illumination gives control over the irradiance and angular aperture of light illuminating a sample. These are controlled in order optimise the spatial resolution and contrast of brightfield images. Köhler illumination has the favourable quality that the source of illumination such as a lamp filament is defocussed in the image. The infinity corrected Objective and Tube lenses are set out in a 4f layout to produce a magnified intermediate image that is registered directly by an Electronic imaging array (Figure 13) or projected onto the retina of a human eye with the use of an eye piece. Between objective and tube lens (termed the "infinity space"), light rays emanating from a point in the sample are collimated. The benefit of this is that auxiliary optical components (such 
as filters) inserted into this space will be defocussed in the image and will not alter the focal distance.

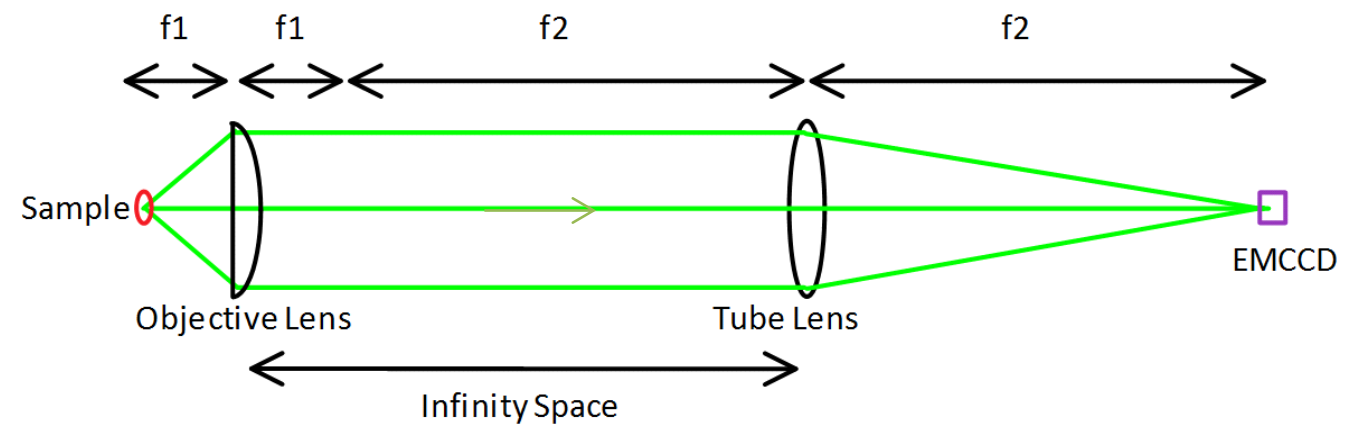

Figure 13 The minimal emission path of a modern optical microscope, an infinity optical system.

The extended wavefront of brightfield illumination is diffracted at two key locations in the microscope. First light is diffracted by the structure of the sample, then at the front aperture of the objective lens. In the case of fluorescence microscopy, radiation is emitted by each sample fluorophore and diffraction occurs primarily at the objective lens aperture. The resulting disturbances to the wavefront propagate through the optical train and ultimately affect the amplitude profile of the focused image. The relative length scale of the wavelength, the sample features, and the objective aperture dictate whether the sample features will be resolved in the image. In brightfield imaging, the angular aperture of the illumination beam also effects spatial resolution and image contrast.

Contrast at the focal plane is created by the constructive interference of undeviated and deviated components of coherent light from the sample plane. The larger the solid angle of light subtended by the objective lens, the higher the proportion of deviated light that is collected and the higher the image contrast will be. As can be 
demonstrated with a diffraction grating, collection of at least the 0 th and 1st orders of diffraction is required for image formation (Murphy and Davidson 2013). For an objective lens with a given angular aperture, the Numerical Aperture (NA) characterises the cone of light collected, taking into account the refractive index of the medium between sample and lens. A high NA indicates high proportion of acceptance and a correspondingly high spatial resolution. The formula for the NA of an objective lens is $N A=n \sin [\theta]$, where $n$ is the refractive index of the medium between lens and coverslip, and $\theta$ is the angular aperture of the lens.

The limited spatial resolution of light microscopy is evident in the airy disc image pattern of an isolated fluorophore. This pattern is described by the point spread function PSF. For a $4 \mathrm{f}$ imaging system with a circular aperture the PSF is mathematically described by a jinc ${ }^{2}$ function (Mertz 2010) and commonly approximated by a Gaussian function. For fluorophores to be resolvable, the minimal spacing between is defined by the Rayleigh Criterion to be that for which the centroid of one airy disc falls over the first diffraction minimum of the other. For a microscope this is calculated with the formula of Ernst Abbe, $d=0.61 \lambda / N A$, where $\lambda$ is the fluorescence wavelength. This is illustrated in Figure 14. 
Two Point Spread Functions Separated by the Rayleigh Limit

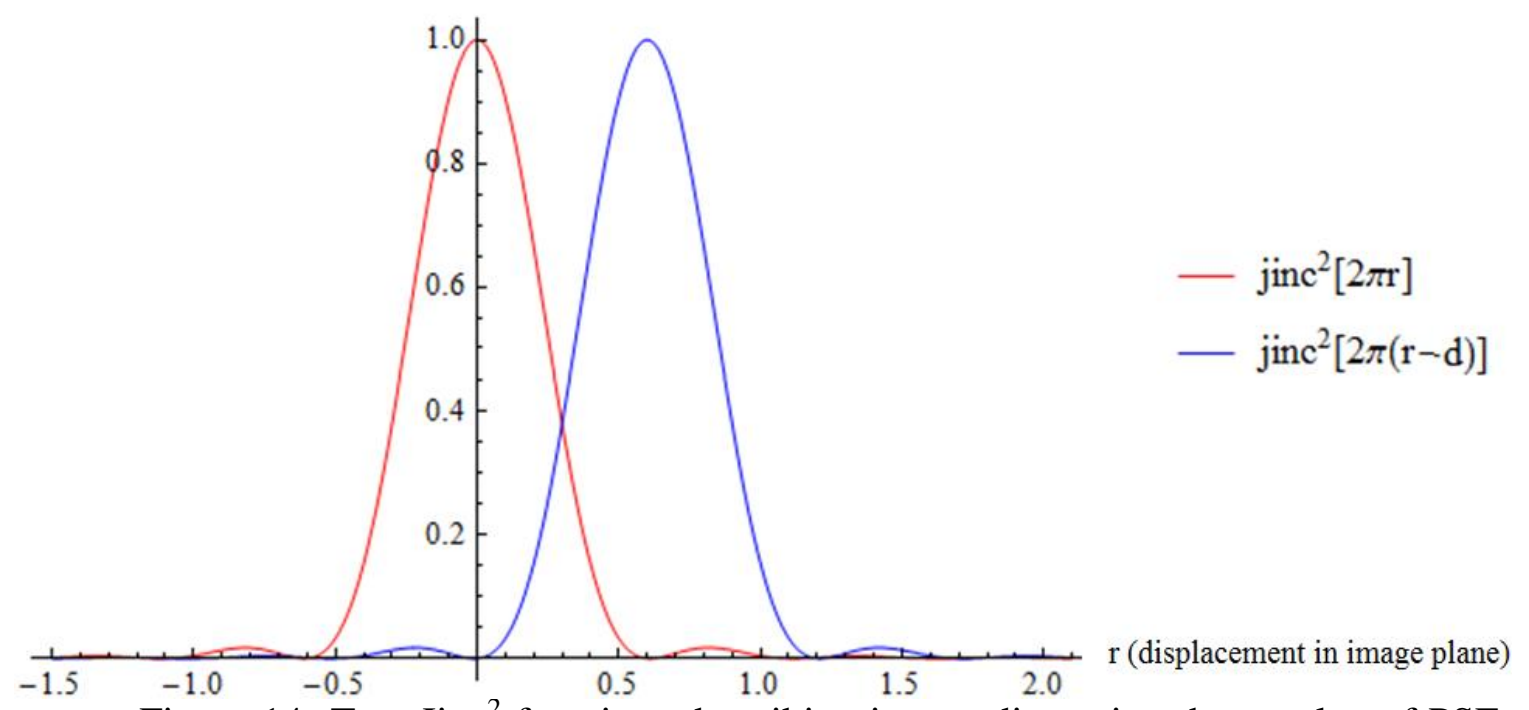

Figure 14 Two Jinc ${ }^{2}$ functions describing in one-dimension the overlap of PSFs separated by the Rayleigh limit.

\subsubsection{Fluorescence Microscopy}

Fluorescence is the radiative emission from an orbital electron as it relaxes to its ground state having been excited to a higher quantum state. Molecules that exhibit fluorescence typically include conjugated systems in which orbitals overlap, leading to decentralisation of electrons and small energy gaps between ground and excited states. The energy imparted on such systems by visible wavelengths may be enough to excite orbital electrons and induce fluorescence. In the GFP molecule for example (Figure 15), fluorescence has been attributed to a central ring structure containing conjugated double bonds. (Lichtman and Conchello 2005). 


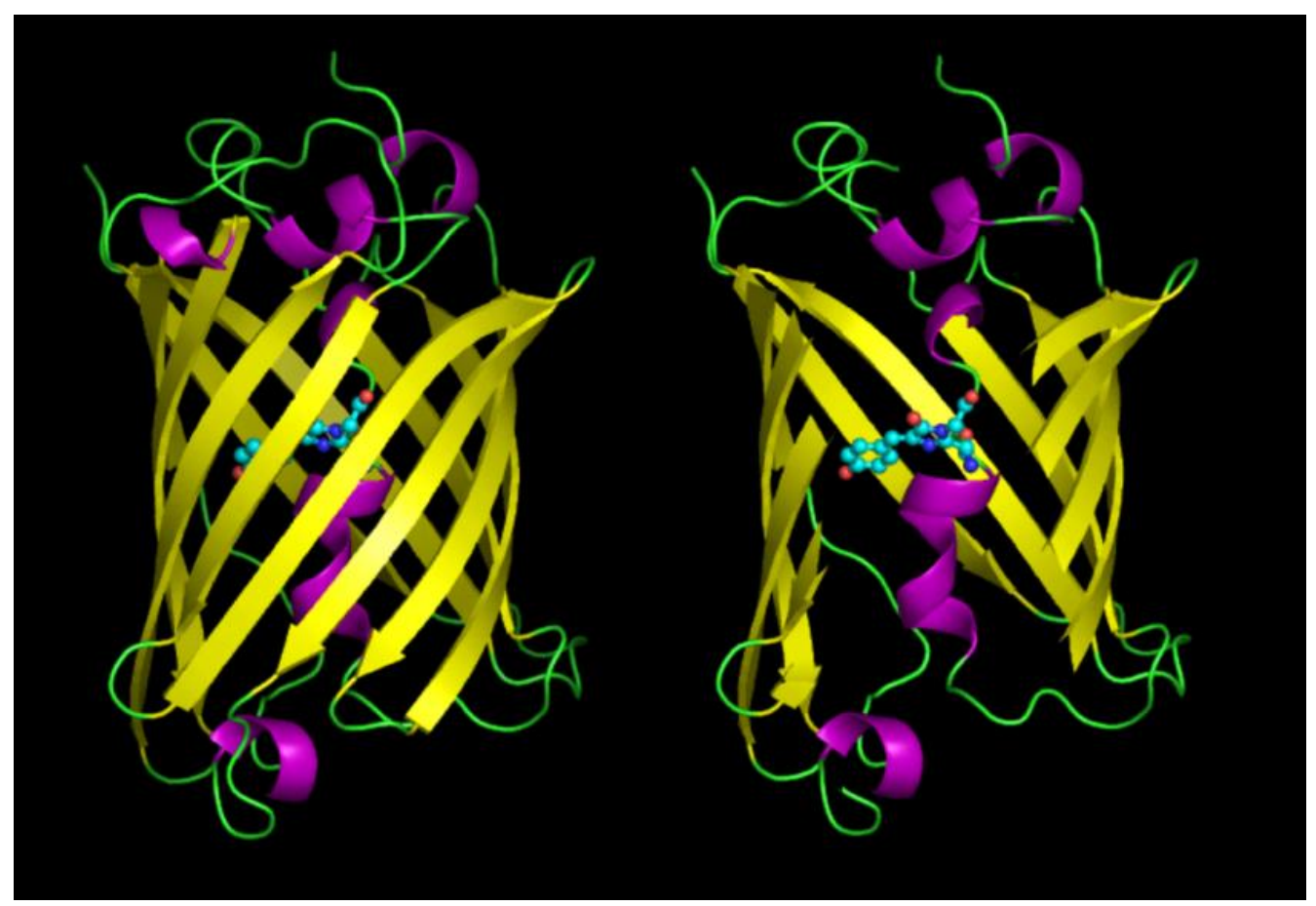

Figure 15 A GFP molecular structures with fluorophore highlighted as ball-andstick. The complete molecule (left) and a cut-away version (right) to show the fluorophore (Keller 2008).

After photonic excitation on the femtosecond timescale, fluorescence emission is just one of the multiple processes of energetic decay. As well as electronic energy states, a fluorophore can be excited into higher vibrational and rotational states. This mechanical energy will then be transferred through collisions to surrounding molecules on the picosecond time scale. Fluorescence emission occurs typically later, on the nanosecond timescale, when the ground electronic state is returned to. Since the energy drop is less than the original excitation energy, the emitted photon is red-shifted (the Stokes Shift). The variety and probability of possible energy transitions results in the signature excitation and emission spectra of each fluorophore type. 
An alternative fluorophore decay path is that of intersystem crossing to the triplet state followed decay to the ground state that if radiative is called phosphorescence and occurs on the microsecond timescale. Further to this, a molecule may experience photobleaching, an irreversible change to molecular structure that ends its fluorescent property. Some key fluorophore energy levels and transitions are illustrated below (Figure 16).

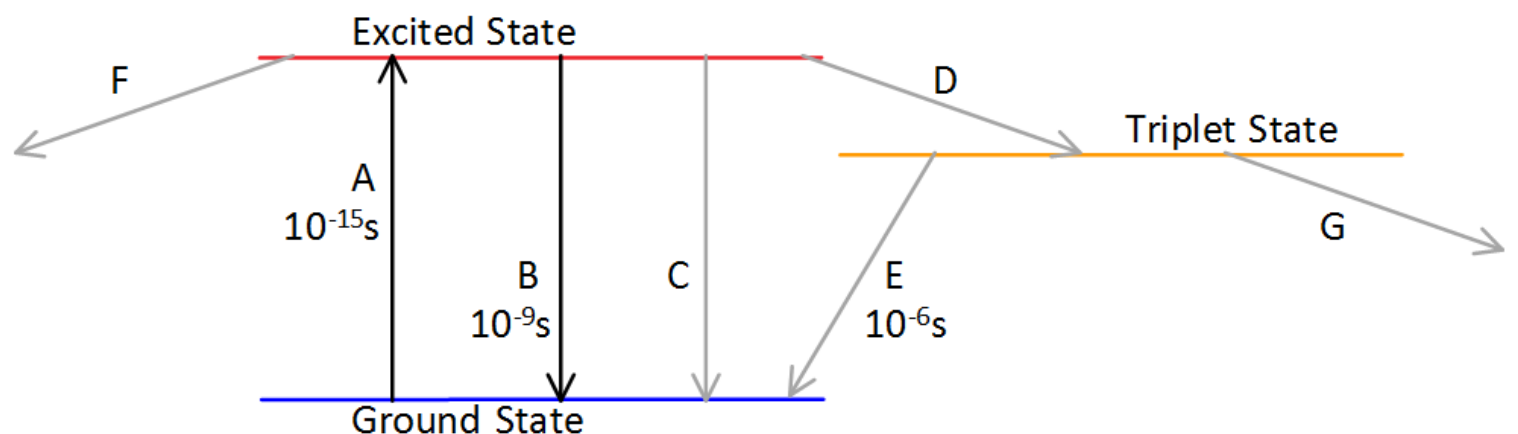

Figure 16 Schematic of the energy states of a three-level system with photobleaching (based on figure from (Mertz 2010)) and times scales of energy transitions as in (Lichtman and Conchello 2005). A) Excitation B) Radiative Decay (Fluorescence emission) C) Non-radiative decay D) Inter-system crossing E) Triplet state decay F) Bleaching G) Bleaching.

The rates of each energy transition can be related mathematically to understand behaviour such as saturation and to predict fluorescence response against time for a given excitation scenario. The system of differential equations associated with Figure 16 (Mertz 2010) was solved and some particular solutions of interest are presented below (Figure 17, Figure 18, Figure 19, and Figure 20). 
Dynamics of a Molecular Three-Level System Occupancy Probability

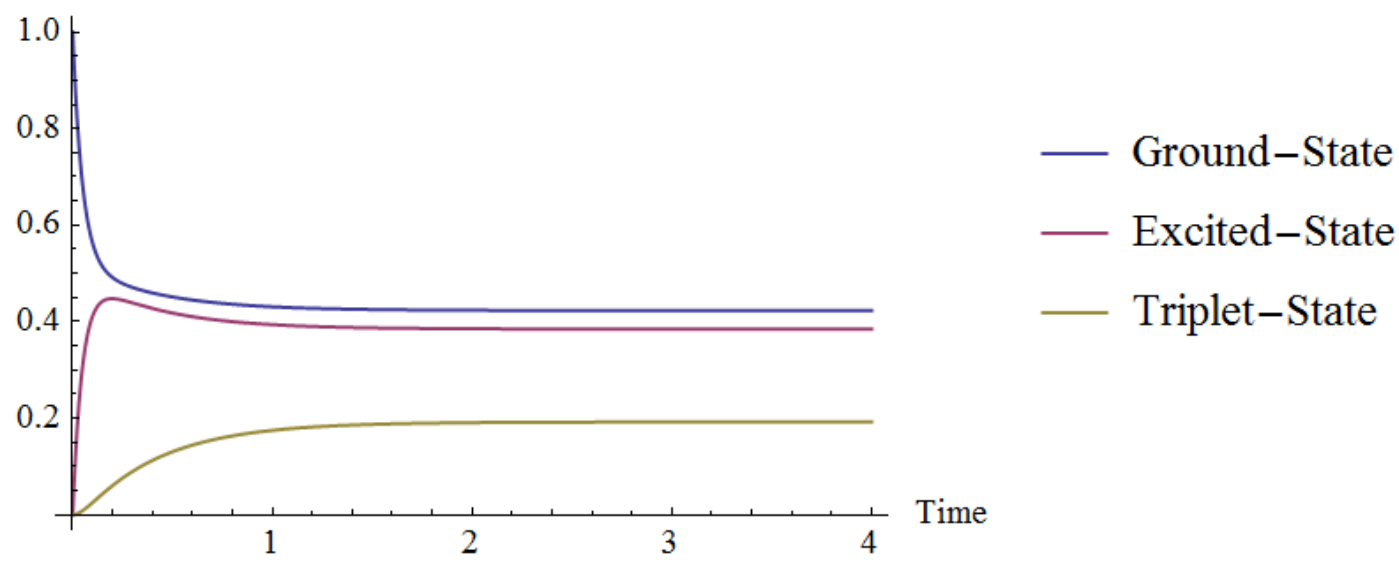

Figure 17 Whithin a closed system (in the absence of photobleaching) a dynamic equilibrium in energy state occupancy is reached.

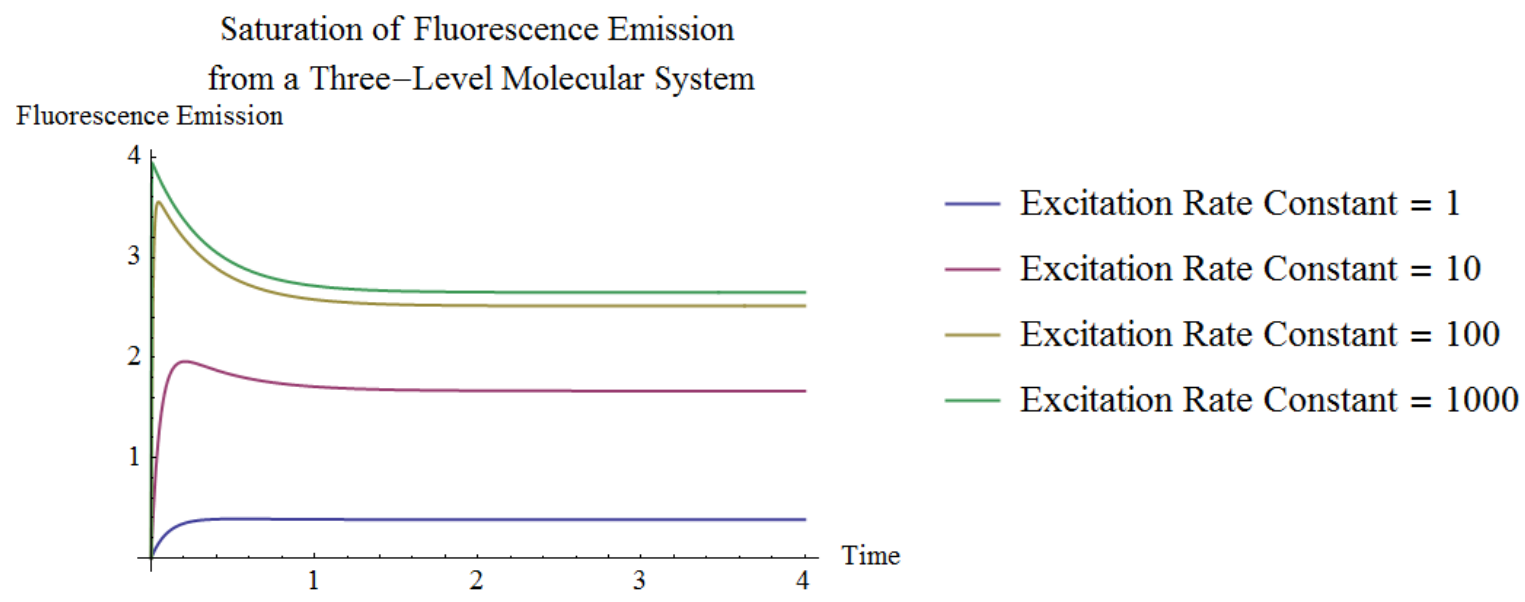

Figure 18 With increasing excitation intensity, the steady-state fluorescence emission tends towards a maximum value determined by the time-scale of radiative decay. 
Dynamics of a Molecular Three-Level System

with Photobleaching

Occupancy Probability

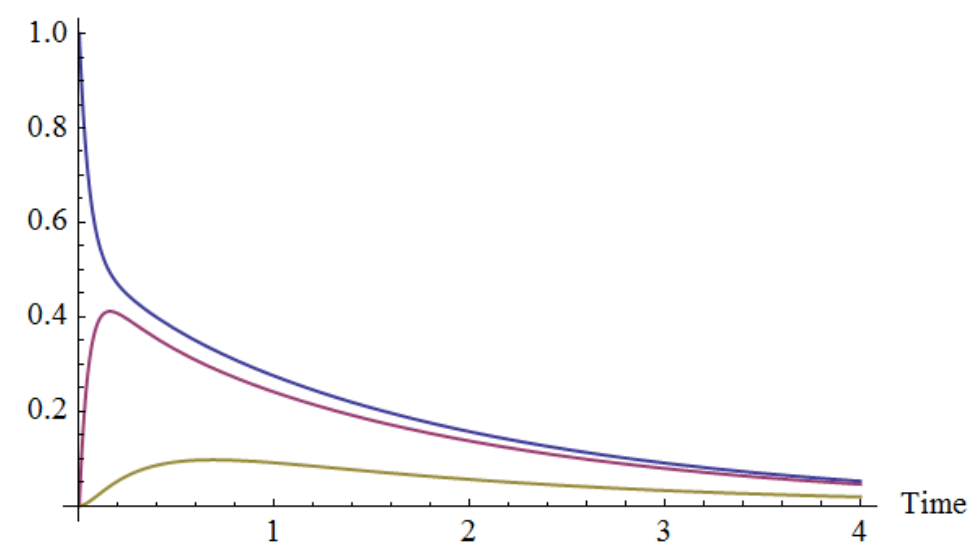

Figure 19 In an open system (in the presesnce of photobleaching) the energy state occupance probability does not reach a steady state.

Saturation of Fluorescence Emission

from a Three-Level Molecular System

with Photobleaching

Fluorescence Emission

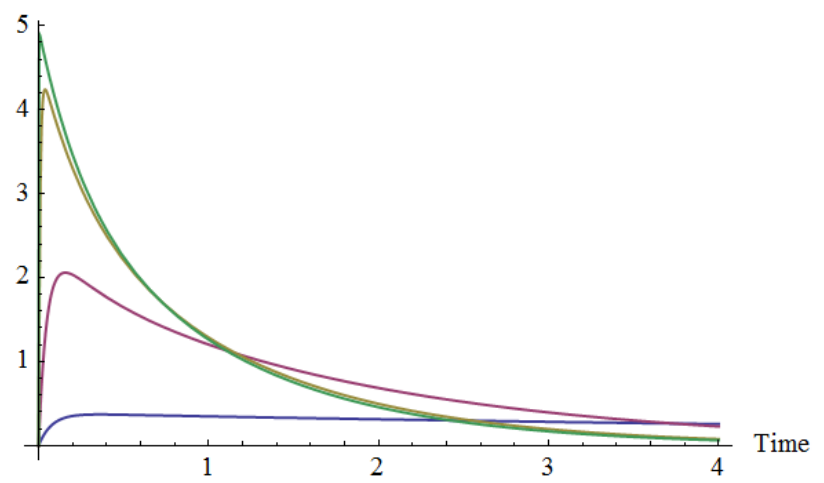

- Excitation Rate Constant $=1$

- Excitation Rate Constant $=10$

- Excitation Rate Constant $=100$

— Excitation Rate Constant $=1000$

Figure 20 With photobleaching, the fluorescence emission is prolonged by illuminating with lower excitation intensities.

The mechanisms and characteristics of fluorescence emission have been exploited to produce numerous quantitative microscopy based techniques. The filtering of illumination light and detection of only Stokes-shifted fluorescence enables a molecular 
level sensitivity unobtainable in scattering based approaches. In scattering based image formation, as with brightfield illumination, the phase of scattered light is critical to the generation of contrast and some illumination coherence is necessary. In the case of fluorescence imaging, the fluorophores themselves are the light sources with an approximately picosecond coherence time scale (Mertz 2010). Since typical imaging time scales will drastically under-sample this coherence, in images of multiple fluorophores only the amplitude and not phase contributions from each fluorophore will be evident.

Three popular modes of fluorescence illumination are Epifluorescence, TIRF and Confocal. Epifluorescence is the most straightforward scheme of in which an excitation light beam follows the optical axis and is focussed on the back focal plane of the objective lens (Figure 21). The beam emerges from the lens collimated and, accounting for scattering effects, will excite the sample evenly along the optical axis. TIRF and Confocal illumination are powerful in that it is possible to illuminate a delimited region of a cell, thus making improvements in SNR and knowledge of spatial localization. Confocal fluorescence microscopy uses a pinhole in the emission path to image only the region of excitation.

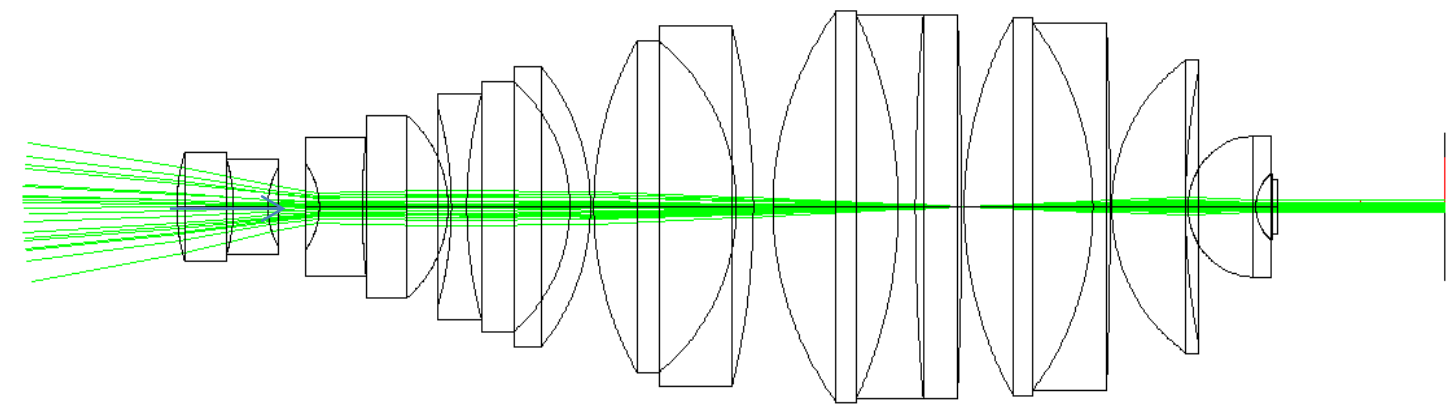

Figure 21 Ray-tracing diagram of the interal optics of the Nikon objective lens and the beam input (from left to right) to achieve epifluorescence illumination of a 
sample. A beam is propogated along the optical axis and focussed on the back focal plane of the objective lense. Description of how figure was rendered is in section 2.2.1.3.1.

Fluorescent samples are often illuminated with laser light due to its monochromaticity and collimation. Most lasers use resonator cavities with cylindrical symmetry from which the fundamental transverse mode is described by a two dimensional Gaussian function. The Beam radius $w$ is defined as the distance from the beam axis where the optical intensity drops to $1 / e^{2}(\approx 13.5 \%)$ of the value on the beam axis. At this radius, the electric field strength drops to $1 / e(\approx 37 \%)$ of the maximum value.

After passing through an aperture, an initially collimated beam will transversely spread as it propagates (Griot 2008). Upon passage through a lens, a Gaussian beam will be transformed into another Gaussian beam with a focussed spot radius described by the equation

$$
W_{F}=\left(\lambda f M^{2}\right) /\left(\pi w_{L}\right)
$$

Were $\mathrm{W}_{\mathrm{F}}$ is the focussed beam radius, $\lambda$ is the wavelength of the beam, $f$ is the lens focal length, $w_{L}$ is the collimated beam radius entering the lens, and $M^{2}$ is the beam quality factor. The beam quality factor, $M^{2}$, is the ratio of the beam parameter product (BPP) for the beam used and the BPP for a beam with a perfectly Gaussian field profile. The BPP quantifies the quality of a laser beam and is calculated as the product of its divergence half-angle and its focussed beam waist radius.

This minimal focussed radius may be smaller than the resolution as defined by the Rayleigh limit. For the objective lens that would be used in the microscope, the Gaussian 
beam waist is plotted for a range of entering beam widths in Figure 22. The spatial resolution worked out for the same lens and wavelength is $205 \mathrm{~nm}$.

Focussed Gaussian Beam Radius against Entry Beam Radius for NA 1.49 Lens with 500nm Light Focussed Beam Radius (m)

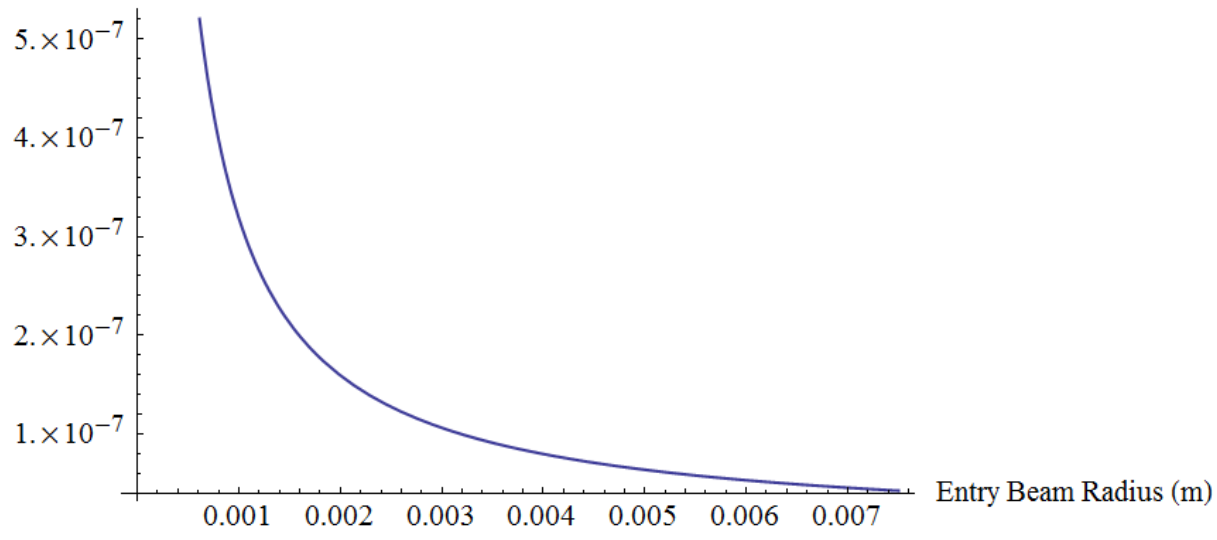

Figure 22 For a wavelength of 500nm and assuming an idealised Gaussian profile (giving a quality factor of $\mathrm{M}^{2}=1$ ).

Often, as in the case of confocal microscopy or in FRAP, the minimal confocal volume is desired. However one may wish to expand the confocal volume to illuminate the entire sample of interest, for example an entire $E$. coli cell in the case of "Slimfield illumination" (Plank, Wadhams et al. 2009). This illumination mode is a simple way to produce a high intensity field to enable high time resolution for capturing cytoplasmic dynamics. Figure 23 and Figure 24 illustrate the back focal plane illumination required to achieve minimal confocal and slimfield sample illumination respectively. 


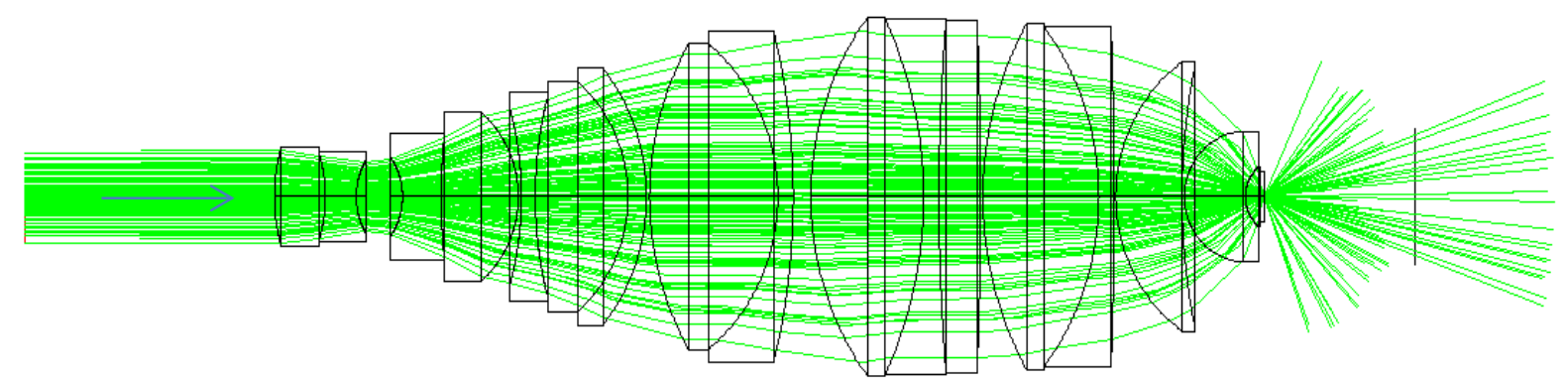

Figure 23 Ray-tracing diagram of the interal optics of the Nikon objective lens and the beam input (from left to right) to produce the minimal confocal volume at the sample. The objective back aperture is over-filled with collimated.

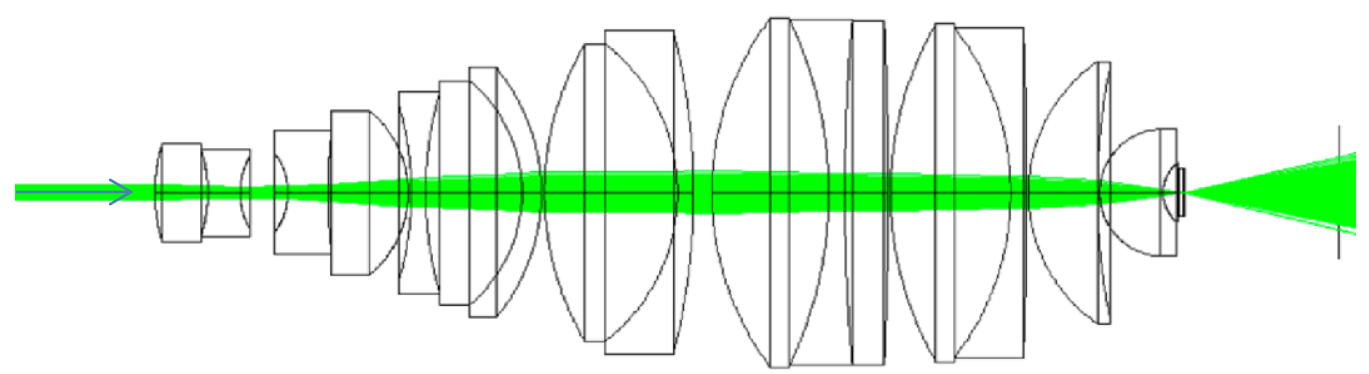

Figure 24 Ray-tracing diagram of the interal optics of the Nikon objective lens and the beam input (from left to right) to achieve slimfield illumination. The objective lens back aperture is under-filled with collimated light.

In TIRF Microscopy an evanescent field is established by illuminating the coverslip from an angle greater or equal to the critical angle (Figure 25). The evanescent wave propagates along the coverslip interface with an exponential decay in field strength in the normal direction (Axelrod 2001). To practically achieve TIRF illumination, a beam is propagated as in epifluorescence but displaced from the optical axis (Figure 26). 


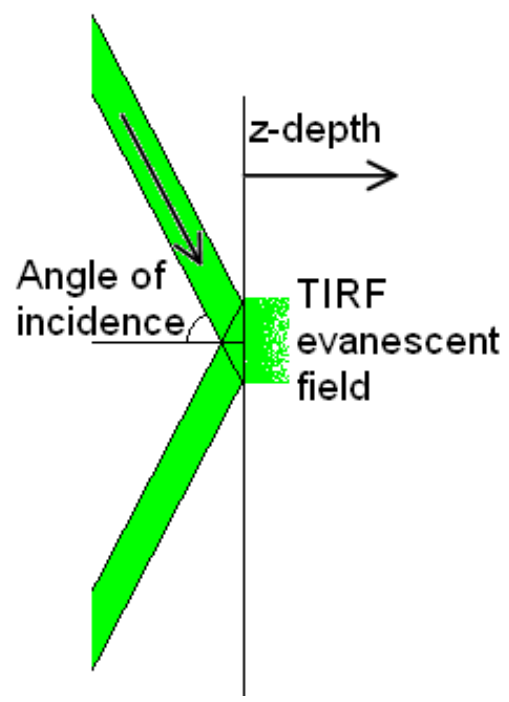

Figure 25 To establish an evanescent field, a light beam illuminates an interface at greater to or equal to the critical angle.

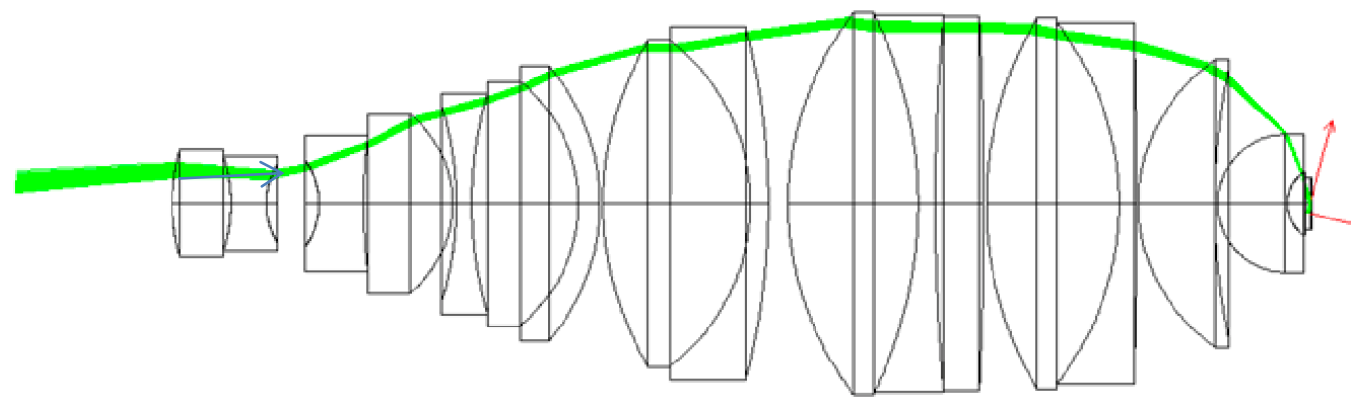

Figure 26 Ray-tracing diagram of the interal optics of the Nikon objective lens and the beam input (from left to right) to illuminate a sample with an evanescent field (TIRF illuminaiton). A collimated beam enters the objective lens back apperture parralel to but displaced from the optical axis.

With increasing incident angle the beam intersection with the coverslip will increase in eccentricity and the relative irradiance can be calculated as $1 / \operatorname{Sec}[\theta]$. In 
Figure 27 the beam intersection with the coverslip-water interface is plotted to illustrate its change with incident angle.
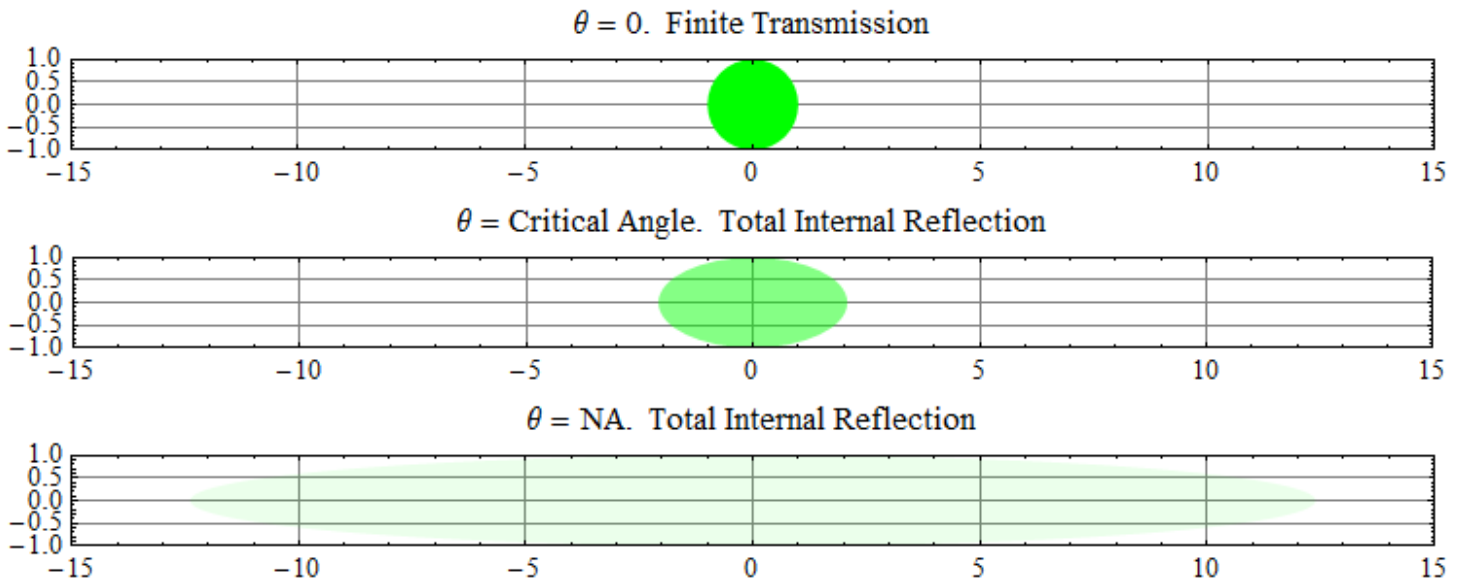

Figure 27 Plots to show how the irradience upon an interface drops with increased angle of incidence. The intersection of a collimated beam with the coverslipwater interface for three angles of incidence is indicated by the green ellipses. The critical angle is calculated from the refractive indices of the coverslip and water and a NA of 1.49 is represented. The increasing transparency of the profile indicates the drop in irradiance with increase in angle of incidence. This drop in irradiance should be considered along with the angle of incidence dependent change in evanescent intensity.

When illuminating a cell, the evanescent field serves to preferentially illuminate the region of the cell that is in touch with the coverslip. The microscope is focussed to produce an image of this region and image contrast is increased since the excitation of fluorophores beyond the focal plane is low. The field decay is plotted in Figure 28. 


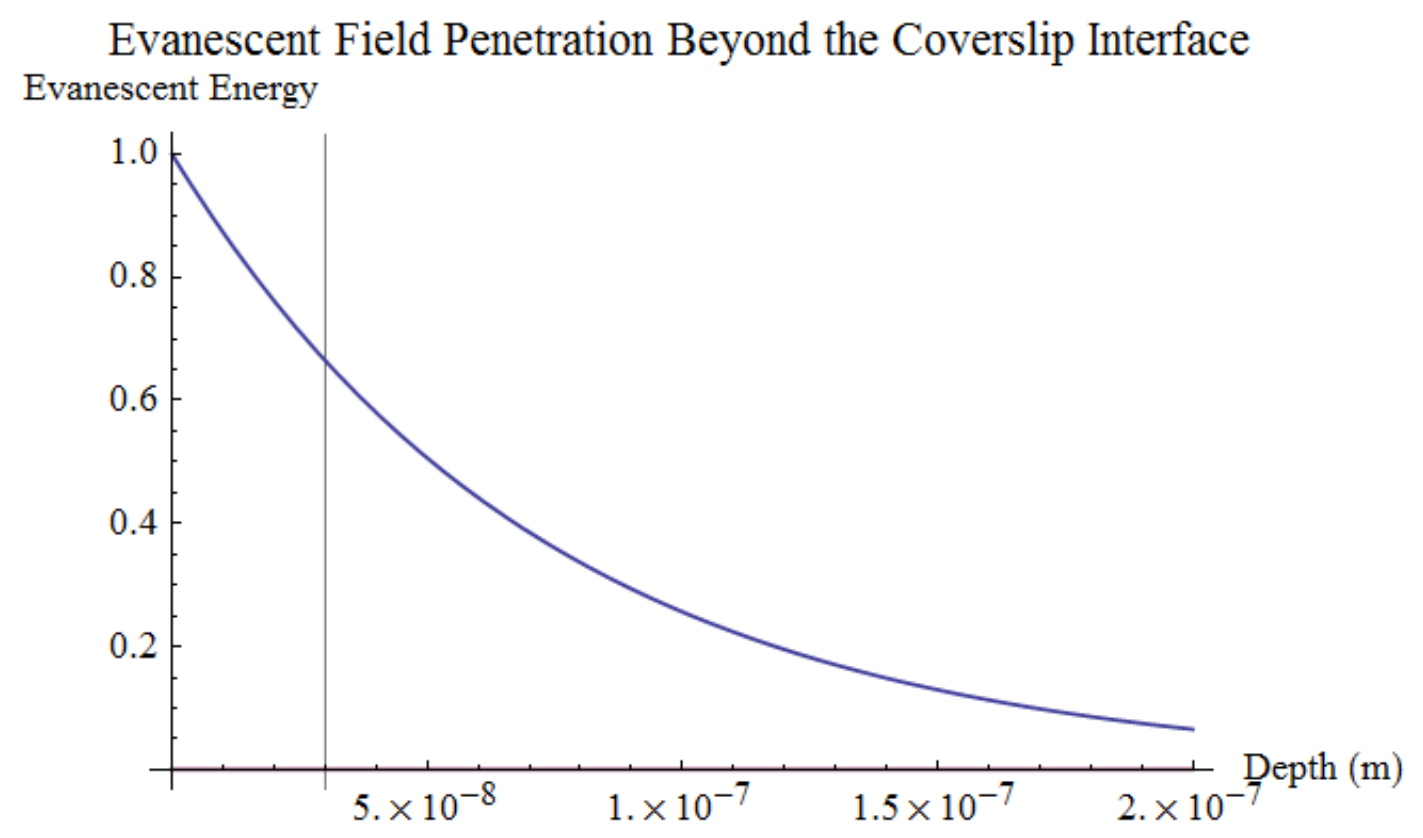

Figure 28 Plot of field strength against depth for 500nm light incident at 1.3 radians. The vertical line marks the depth of the interface between cell envelope and cytoplasm of an E. coli cell.

When a photon impinges on an electron in a fluorophore, they interact via the Lorentz force from their electric fields. The resultant redistribution of molecular electrons depends on the orientation of the fluorophore transition dipole moment. A photon will most likely be absorbed if its electric field vector is parallel to the fluorophore transition dipole moment. When the energy is released it will be polarised as the dipole transition moment and exhibit a radiation pattern that is strongest in the plane perpendicular to the moment. Within near-field proximity to a planar interface, this dipole radiation pattern is further complicated and it is not trivial to calculate the proportion of fluorescence that will pass across the interface (Lukosz and Kunz 1977).

If the dielectric interface is assumed to have no effect on the radiation pattern and the fluorophore is assumed to be rotationally mobile in the time scale of image exposure, 
fluorescence emission can be approximated to be isotropic. By considering the proportion of the total solid angle subtended by light entering the objective lens, an NA of 1.49 can be calculated to collect $46 \%$ of isotropically radiated fluorescence.

The magnified image is ultimately formed upon the pixelated surface of an electronic image sensor. These pixels must be suitably small to sample the image features and avoid aliasing. As dictated by the Nyquist limit the radius of the PSF should be spanned by no less than two pixels to retain diffraction limited spatial resolution.

For a given projected image with its associated shot noise and artefacts, the ability to attain single-molecule sensitivity is limited by the sensitivity and inherent noise of the imaging sensor. For high sensitivity electronic imaging either an Electron Multiplying Charge Coupled Device (EMCCD) or a Complementary Metal Oxide Semiconductor (CMOS) sensor is typically used. The sensor response can be modified by the parameters controlling image acquisition including the exposure time, gain settings, and the number of digitization levels. 


\subsubsection{Architecture DeVelopment}

\subsubsection{The Engineering of Systems}

Stepping outside the field of experimental science, a broad array of literature was read on the subject of system development. It became apparent that there are diverse disciplines and points of view in the development of technological systems. There is also a diversity of definitions to related words such as "architecture" and "design". A principle common to most approaches is to develop an abstract description of the problem to be solved. From this description, relevant solutions from other systems may be identified. In synthesis of a technical system it is also suggested that multiple solutions are generated and that one uses design tools to systematically explore possibilities and to make best use of ones capacity for intuitive thought (Mandenius and Björkman 2011).

The creation of system structure is generally termed Architecture. An example of an architectural decision was to use the TIRF scheme of microscopy to image membrane bound proteins. The review of section 1.1 was written to assist the key architectural decision of selecting an appropriate single-molecule technique from the diversity that now exists (Harriman and Leake 2011).

The challenge of architecting highly complex systems can be made tractable by use of Heuristics, abstracted principles of guidance learned through multiple concrete experiences. Though heuristics are qualitative, their use can have a profound effect on 
the outcome of a development effort. The following are examples of heuristics that are seen to be particularly pertinent (Maier 2009):

a) Don't assume the initial statement of the problem is the best, or even the right one.

b) In partitioning, choose the elements so that they are as independent as possible; that is, choose elements with low external complexity and high internal complexity.

c) The eye is a fine architect, believe it. Simplify. Simplify. Simplify.

d) Build in and maintain options as long as possible in the design and implementation of complex systems. You will need them.

An interesting parallel to physical engineering is the huge and rapidly developing field of Software Engineering. The diverse experiences from attempts to build highly complex and efficient systems have led to a fascinating body of knowledge on best practices and powerful conceptual frameworks. A currently pervasive approach of software engineering is the use of object oriented analysis and design (OOAD). In OOAD a process is followed in which a system is modelled as a group of interacting objects. Given the objects and functionalities derived, a generalised structural pattern can be applied, an architectural scheme that through vast experience has been found to be effective. Design patterns can be seen in physical engineering also, for example the layout of an infinity corrected optical microscope.

There are debates as to whether software systems should be designed primarily with a ground-up focus on detail (Martin 2008), or from a high level structural view as 
with the Unified Modelling Language (UML). UML is a graphical modelling language with which one can map out programme structure and functionalities in a hierarchical manner prior to coding. There are compelling arguments that say the software code itself should be considered the model, and that any prior modelling would be a waste of resources and error prone due to ignorance of coding details.

In physical engineering the situation is different since the cost and lead time of components can prohibit the ability to develop and evaluate multiple alternative solutions. In the last decade an extension to UML has been developed called SysML which is a general purpose modelling language for systems engineering (Friedenthal, Moore et al. 2011). SysML attempts to cover every aspect of system development in an integrated semantic manner. Rather than using the traditional document centric approach, all development information is assimilated in an internally consistent model that can be quantitatively evaluated. It is hoped that this approach will enable greater efficiency, clarity, collaboration, and capabilities in system development. The SysML approach is generically called Model Based Systems Engineering (MBSE). It is yet to be known whether MBSE will succeed the document centric approach. If it does, this may indicate its usefulness in scientific endeavour.

In the basic systems engineering process, primarily the needs of all stakeholders are analysed in order to identify system goals and measures of effectiveness in achieving the goals. Next the required system functions, interfaces and performance are specified. Separable system components are then developed and from these alternative system schemes are developed. The solutions are evaluated against each other in terms of how they meet the requirements, meet effectiveness measures, and satisfy stakeholder needs. 
Though one might not want to model every aspect of an experiment, there may be benefits in the early stage of project development. Early project decisions are found to have very great effect on project outcomes since they define project scope and direct the use of resources (Willcox and de Weck 2004). Methodologies therefore are being developed to include decision support simulation as far upstream in the development process as possible. Such simulation models combine the physical descriptions of the technology to be developed with the practical considerations of developing such a technology.

Rather than using a totally general development approach, another option is to use a software tool corresponding to a specific job type. Such tools typical present streamlined processes through user friendly interfaces and facilitate the use of general computational techniques with domain specific information. Zemax is a widely used tool in the field of optical system design that enables simulation of both geometric and physical optics in three-dimensional space. It is possible to precisely define optical components and simulate system response to illumination. Much knowledge of optical theory and available optical components is internalised within the software and one can apply built-in techniques such as optimisation, sensitivity analysis, tolerance analysis, and thermal expansion analysis with relative ease. All common optical analyses can be carried out and the results plotted in various graph types.

Though such software is purpose build for optical system design, its task appropriateness should not be assumed and it requires some experience to utilise effectively. A basic principle is that one should do as much as possible without such a tool until it is needed to answer to a specific question. However, from the desire to learn 
what might be applicable from the world of engineering, Zemax was used in a partially exploratory manner in this project.

\subsubsection{Purpose of the Microscope}

Typically experiments are built for the sole purpose of answering one clear question with the highest performance possible. While this is the most direct approach to answering a scientific question, laboratory resources such as space, time, and equipment are often limited. There can be a need to serve multiple experimentalists with differing functional requirements. Given the number of cannibalised microscopes lying around the department, it was seen as worthwhile to explore whether a microscope could be made that efficiently used resources, could be user friendly enough to be used and maintained not just by the builder, and could enable the range of functionality that diverse future research projects could require. Beyond providing multiple alternative functionalities, the goal was to enable novel experiments through the ability to simultaneously apply multiple functionalities on a sample. The range of desired live-cell experimental techniques is presented below in Figure 29. It was planned that further capabilities could be integrated into the microscope including Infrared Laser Tweezers to exert force, and Back focal Plane Interferometry for motion detection with ultrahigh temporal resolution. To achieve the range of desired experiments the necessary illumination modes were then determined, as presented in Figure 30. 


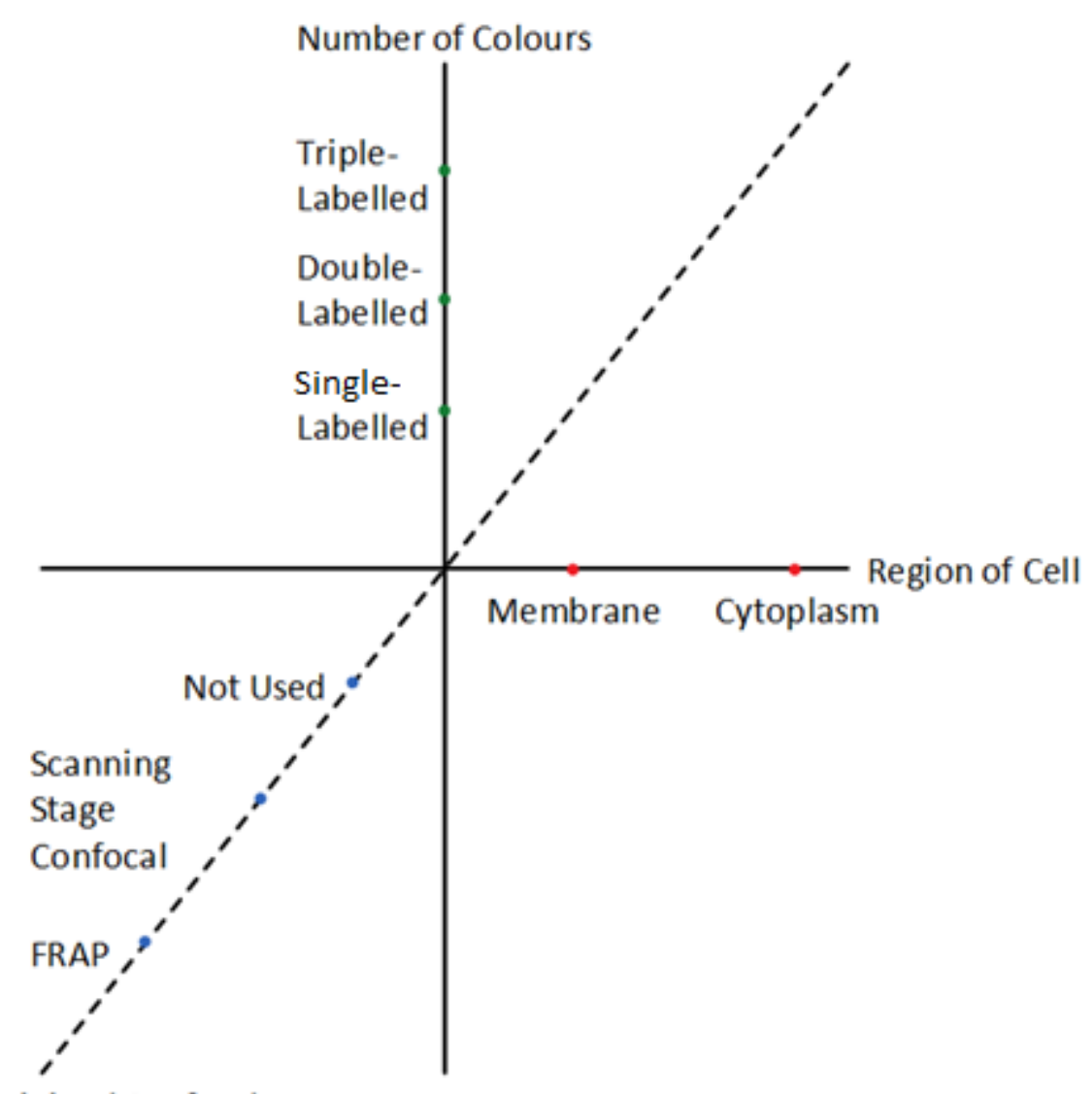

Use of Minimal Confocal

Figure 29 A representation of the desired Experimental-space. It was desired that the microscope would be able to achieve any experiment characterised as fitting into a point in the three-dimensional space defined above. For example, it should be possible to do an experiment imaging the membrane of a cell using two spectrally distinct fluorescent labels and applying the FRAP technique. 


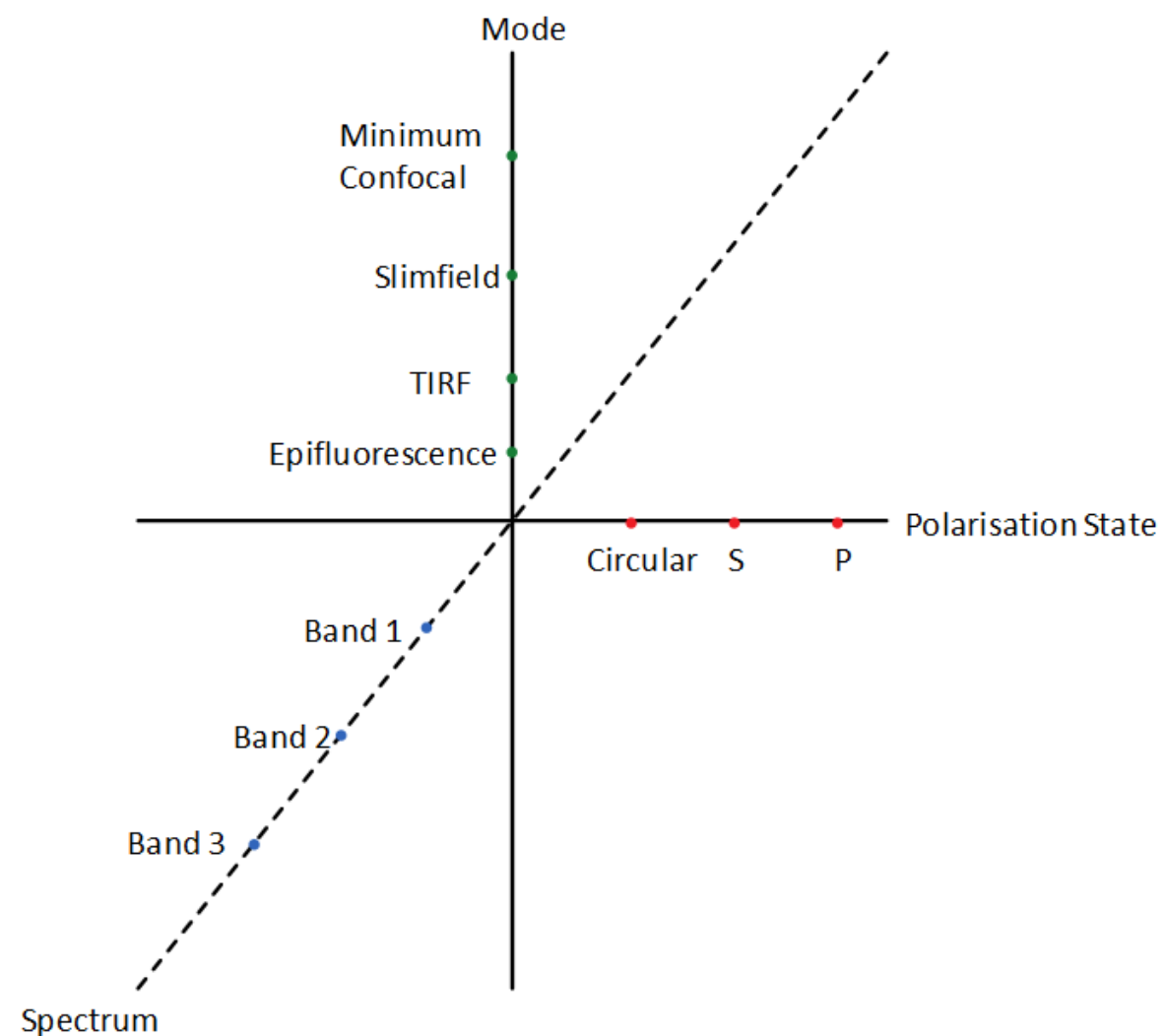

Figure 30 A representation of the desired Illumination -space. It was desired that the microscope would be able to achieve any illumination configuration characterised as fitting into a point in the three-dimensional space defined above. For example, it should be possible to simultaneously apply S-polarised Epifluorescence illumination of three spectrally distinct bands.

A fluorescence microscope can conceptually be split into the illumination path and emission path. From the Illumination path, light beams are generated and sent through the back aperture of the objective lens to irradiate the sample. In many microscopes it was observed that laser beams of different wavelengths were combined and then controlled as one prior to entering the back aperture of the objective. This gave 
the simplicity of only modifying one combined beam but imposed constraints on possible illumination schemes.

It was conceived that novel future experiments could be enabled by having independent control over the trajectory, expansion, attenuation and polarisation of the beams of each wavelength. An example of a conceived novel experiment that would be enabled by the independent positioning of confocal volumes is presented in Figure 31. Furthermore, in TIRF microscopy the evanescent field penetration depth is dependent on wavelength and it may be desirable to equalise it. Independent beam control would also however create a burden on the experimentalist in the form of a higher alignment and maintenance workload.
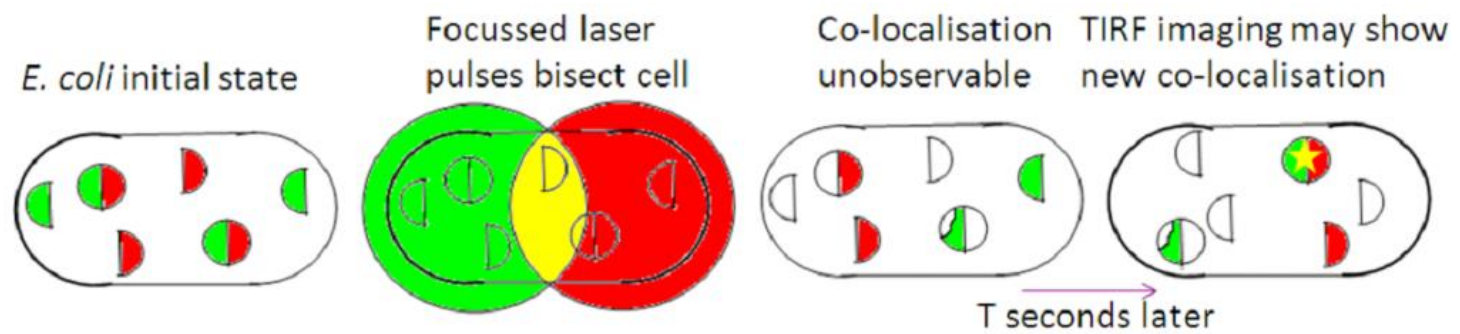

Figure 31 A conceived approach to probing cluster assembly dynamics.

Photobleaching different fluorophores in each half of the cell means that any colocalisation observed afterwards must have occurred since the photobleaching.

\subsubsection{Optical Patterns}

Steering of light beams can be achieved through the mechanical reorientation of a mirror or lateral translation of a lens, or through acousto-optical deflection in a crystal. While the optomechanical approaches are typically cheaper, simpler and result in less 
beam attenuation, the acousto-optical manipulation of light can be carried out on much shorter time scales.

Beam Attenuation can be achieved with absorptive and reflective filters, polarisation based splitting, or through acousto-optical modulation. A very simple laser beam attenuation pattern utilises a sequential combination of an $\mathrm{OD}=2$ absorptive and an $\mathrm{OD}=0-2$ variable reflective neutral density filter. By rotating the reflective filter and choosing whether to include the absorptive filter transmissions from $0.01-100 \%$ could be accessed.

It was also desired that the microscope would have the capability to enact very rapid automated changes in attenuation. With stroboscopic illumination one can precisely localise molecules that would otherwise be blurred for a given exposure time (Xie, Choi et al. 2008). This capability is achievable through use of an Acousto Optical Modulator. In the experimental images in Figure 32, one can see the un-diverted beam with the acoustic field off, and multiple diverted orders of diffraction with the acoustic field on.
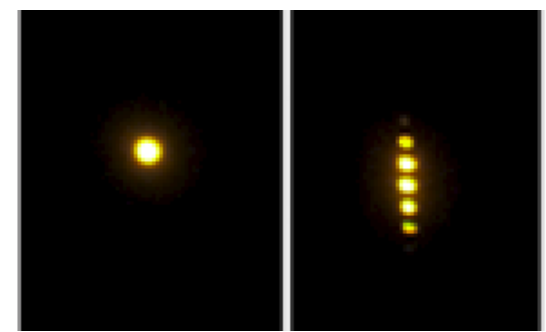

Figure 32 (left) A laser beam passing through an acousto optical modulator with no acoustic field applied. (right) The multiple orders of diffraction resulting from application of the acoustic field.

Beam Expansion can be achieved with two basic two-lens patterns, the Galilean and Keplerian telescopes. For a given expansion ratio, while the Galilean pattern may 
requires a shorter path length, the Keplerian pattern was used since it produced a conjugate image plane at which spatial filtering could be applied.
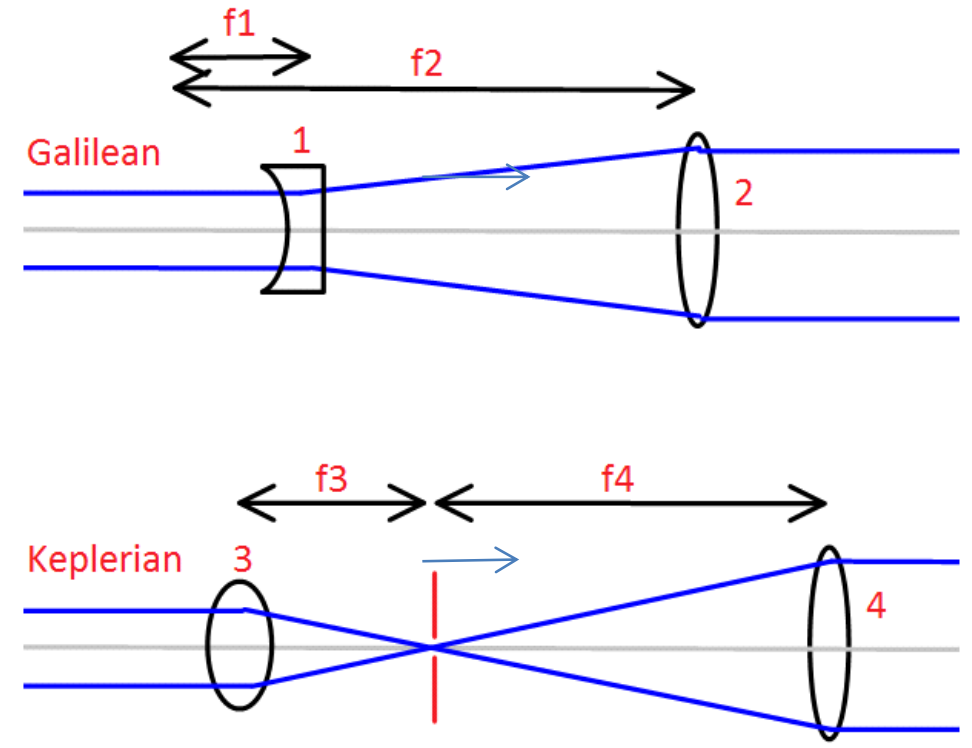

Figure 33 Alternative techniques of beam expansion. Using the Keplerian technique, a spatial filter can be integrated.

In a supercontinuum laser, a mode-locked laser pumps a highly non-linear fibre with picosecond IR pulses. Interaction with the highly non-linear fibre structure results in spectral and temporal broadening of the output beam (Figure 34 left). From a supercontinuum source one can select specific spectral bands to suit the particular fluorophores to be imaged. If one uses a multi-band absorption filter to select the bands (Figure 34 centre), the bands need not be co-aligned unlike when using multiple monochromatic lasers.

A decided philosophy of the bespoke microscope was that one could achieve maximally independent manipulation of each path. This may be preferable if one wished to compensate for chromatic effects or to direct beams to separate points in the sample. 
A pattern of dichroic mirrors was created that would split the supercontinuum beam into separate colour channels (Figure 34 right). This pattern enabled selection of three adjacent bands of arbitrary width and location from a broadband spectrum using dichroic mirrors. Alternative methods exist for the selection of spectral components from a supercontinuum beam. The light could be separated with a prism or diffraction grating, or an acousto-optical filter could be used to sequentially select alternative bands at a sub millisecond rate.
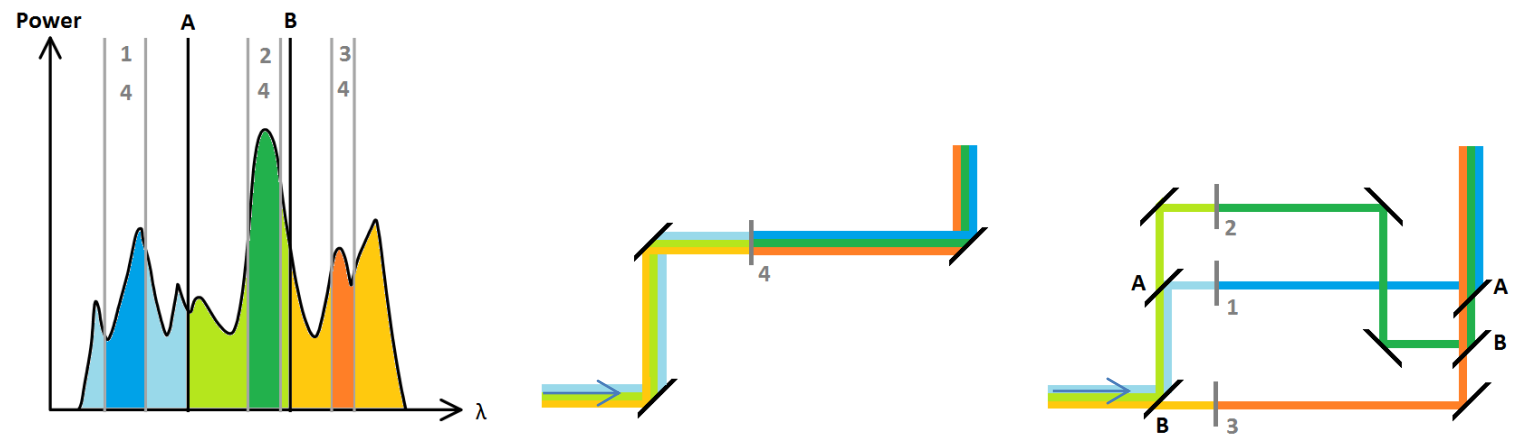

Figure 34 Left) Power Spectral density of a supercontinuum source and the cutoff wavelengths of the dichroic mirrors labelled with letters A and B, and Transmitted bands of absorption filters labelled 1 to 4 . Centre) a configuration dichroic and absorption filters with the specified respective cut-off wavelengths and transmission bands and absorption filters that separates and recombines the beams enabling independent colour channel control. Right). The use of a single emission filter with multiple transmission bands and control of beam as combined colour channel.

Polarisation can also be used as a basis for the splitting and combination of light. Figure 35 below is a schematic of a very straightforward scheme that uses polarising beam splitting cubes. 


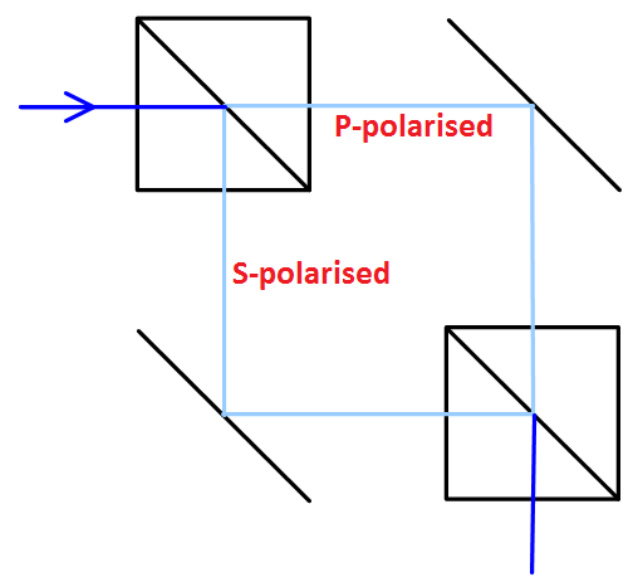
beam.

Figure 35 Polarising Beam Splitter Cubes used to split and recombine a single

In the emission path, a field stop, an aperture at an intermediate image plane, can be used to crop the image allowing projection onto adjacent regions of a sensor. To reduce artefacts associated with optical interfaces, mirrors and filters should be placed either in Fourier planes or as far from the focus of a converging beam as possible.

Though optical elements add functionality, they can attenuate and add undesired artefacts to the original beam. It is therefore preferable to minimise the number of optical interfaces. This minimal number must retain the ability to align each beam with at least two components for beam steering.

\subsubsection{Microscope Layout}

Appropriate optical patterns were combined to make the microscope architecture presented in Figure 36. As desired, the architecture could conceptually enable each experiment type through the ability to apply three simultaneous forms of illumination. 


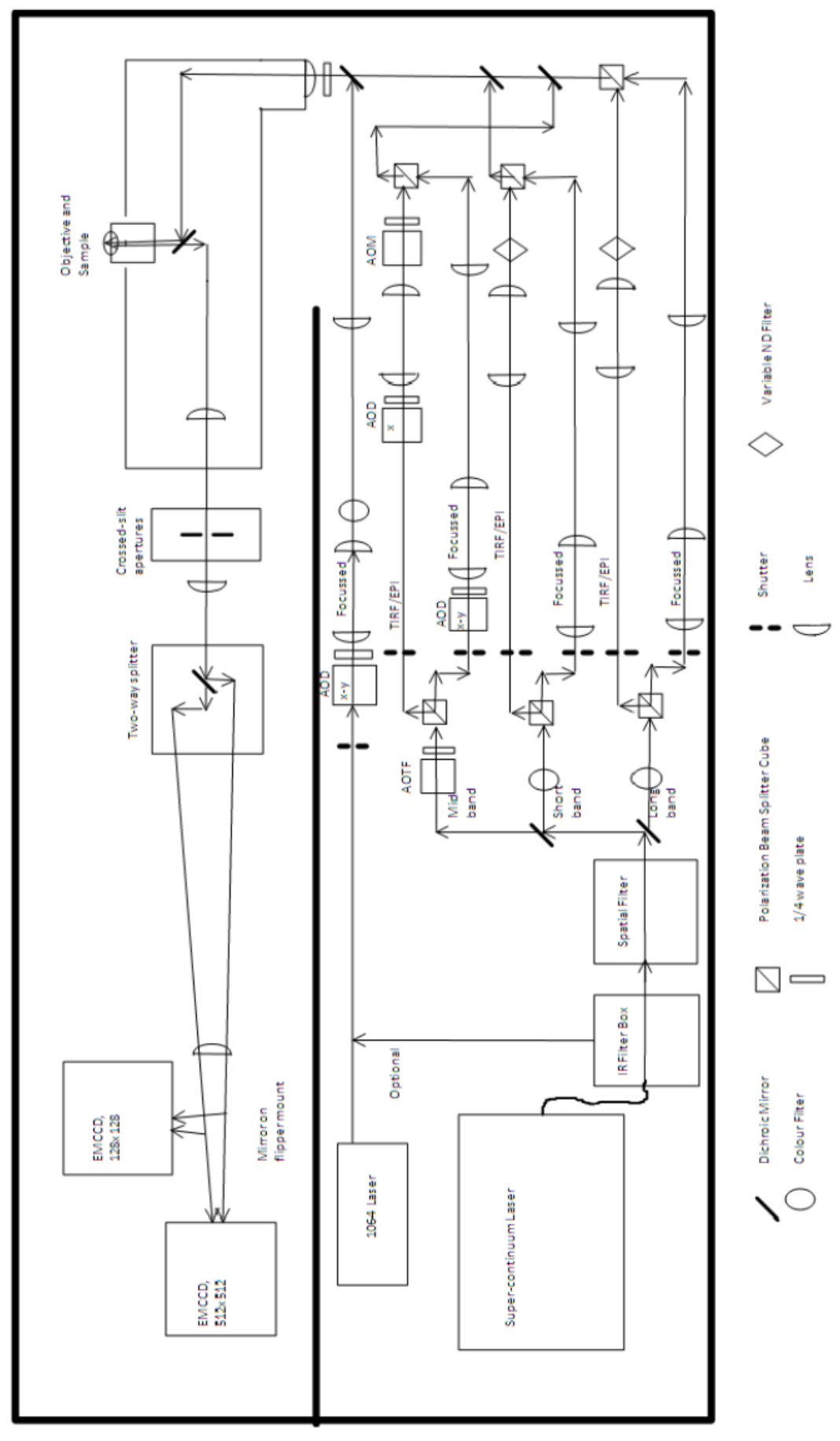

Figure 36 A schematic of the microscope as designed prior to construction. Design modifications were made during the process of building. 
The illumination path of the microscope incorporates laser-tweezers with three colour channels selected from a supercontinuum laser source. Each colour channel has independently controllable S- and P-polarised beams that are either collimated or focussed at the sample with changeable expansion and attenuation factors. The emission

path enables single or double high-sensitivity image formation on a $128^{2}$ EMCCD suited to high frame-rates and imaging of E. coli, or $512^{2}$ EMCCDs with a large field of view suited to imaging larger eukaryotic cells.

Due to magnetic mounts in the emission path, reproducible Image splitting would be achievable based on polarisation or spectral content. The instrument could be seen as a Swiss-army-microscope for single-molecule live-cell imaging. Different excitation path configurations enabled by swap-able dichroic mounts would give the option, of splitting the beam into distinct spectral bands, or manipulating it un-split. If un-split, the beam would be controllable through opto-mechanics or acousto-optics.

\subsubsection{Microscope Construction}

Prior to construction of the microscope, it was necessary to furnish a new laboratory. Some equipment had already been ordered including a Nikon Inverted microscope, a supercontinuum laser, an EMCCD, and some acousto-optics devices. Further equipment was identified and ordered including two Vibration Isolating optical tables, opto-mechanics, computers, and peripheral mounts. All equipment was unpacked and organised within the new room to enable development of two separate optical setups and use of a wet lab space. 
Initially the useable space on the 2-by-1m vibration Isolation table was surrounded by anodised aluminium side walls. The walls contained the maximal space within which the microscope should fit and would ensure that high intensity horizontal light beams could not escape into the room. Later, a foam board lid was constructed to stop dust particles and stray light from entering the space.

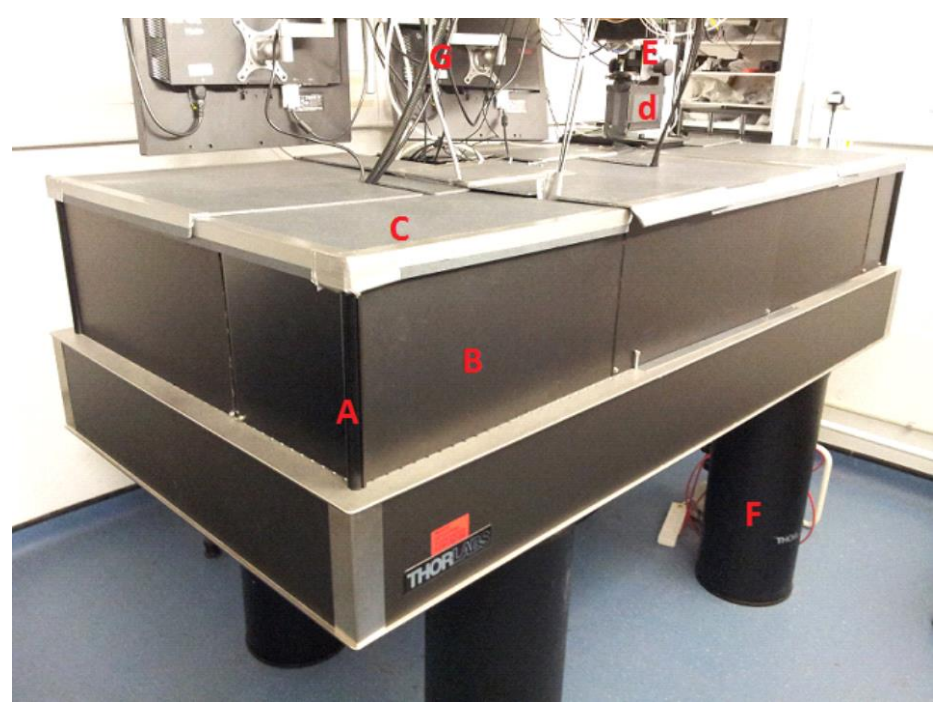

Figure 37 A) Aluminium pillar to constrain anodized aluminium sheets. B) Anodized aluminium sheet (600-by-300mm). C) Black Foam Board top piece, D) Black foam board shield of light emerging from objective lens. E) Nikon Microscope Köhler illumination pillar. F) Pneumatic vibration isolation leg. G) Cables leading from components on the table to components on a gantry above.

It was initially thought that, within the given Nikon microscope body, light from the sample could either be directed $100 \%$ towards the eyepiece, or $20 \%$ to eyepiece and $80 \%$ to the side port. Based on this a decision was made that the $100 \%$ from the eyepiece port should be sent to the high sensitivity EMCCDs for single-molecule detection. This would result in an atypical emission path layout. A track was marked in the form of a $U$ inside the walls and the microscope was bolted into a position roughly half way around 
the $U$, giving equal space to the future illumination and emission paths. The piezoelectric $\mathrm{x}-\mathrm{y}-\mathrm{z}$ nano-translation stage was mounted upon the standard mechanical translation stage of the microscope.

Ultimately a stage top incubator would be installed in the nano-translation stage to enable temperature and gas control. Due to the size of the incubator it would preclude use of a high NA condenser lens. A modified condenser was made from an achromatic doublet with a much lower NA. Use entailed a loss of spatial resolution, but it could irradiate the sample adequately for reasonable image contrast.

Viewing through the eyepieces, the objective lens was focusses on a sample of $1 \mu \mathrm{m}$ latex beads. A low cost (but sensitive) CCD was positioned at the side port intermediate image plane and locked in its $\mathrm{z}$ position (leaving its lateral $\mathrm{x}$ and $\mathrm{y}$ free to explore image with its field of view). The eyepiece turret was then removed so the EMCCD emission path could be built.

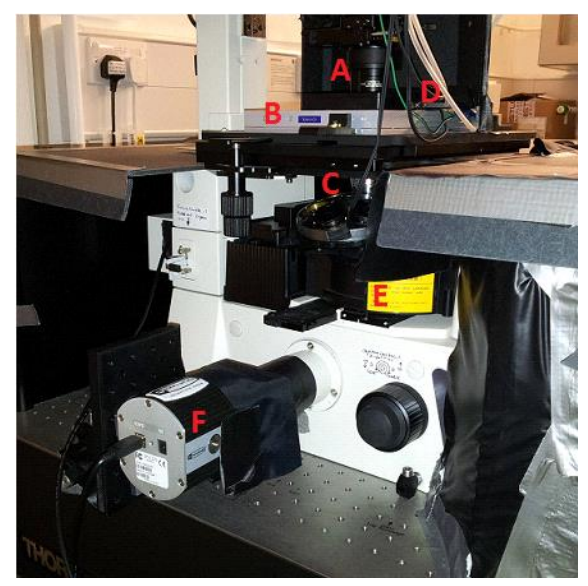

Figure 38 A) Modified condenser lens for compatibility with stage top incubator. B) Nano-positioning stage. C) Objective Lens. D) Stage-top incubator. E) Port for accessing filter blocks. F) CMOS camera mounted on $\mathrm{x}-\mathrm{y}-\mathrm{z}$ translation stage. Previously a CCD was positioned here. 
The EMCCD emission path was developed incrementally through a combination of calculation and experimental trial and improvement. Primarily the beam emanating from the eyepiece turret was characterised since some optics in the Nikon body were unspecified. It was necessary to maintain a beam diameter smaller than the 1 inch diameter of the available optical components. All lenses added to the emission path were Achromatic Doublets with anti-reflection coating for the visible range. Focal lengths were selected that resulted in an image magnification of 350X, with pixels covering 69 and 46 nanometres when using the $128^{2}$ and $512^{2}$ pixel EMCCDs respectively. This resulted in around 4 pixels per PSF central spot radius, double the necessary Nyquist frequency.

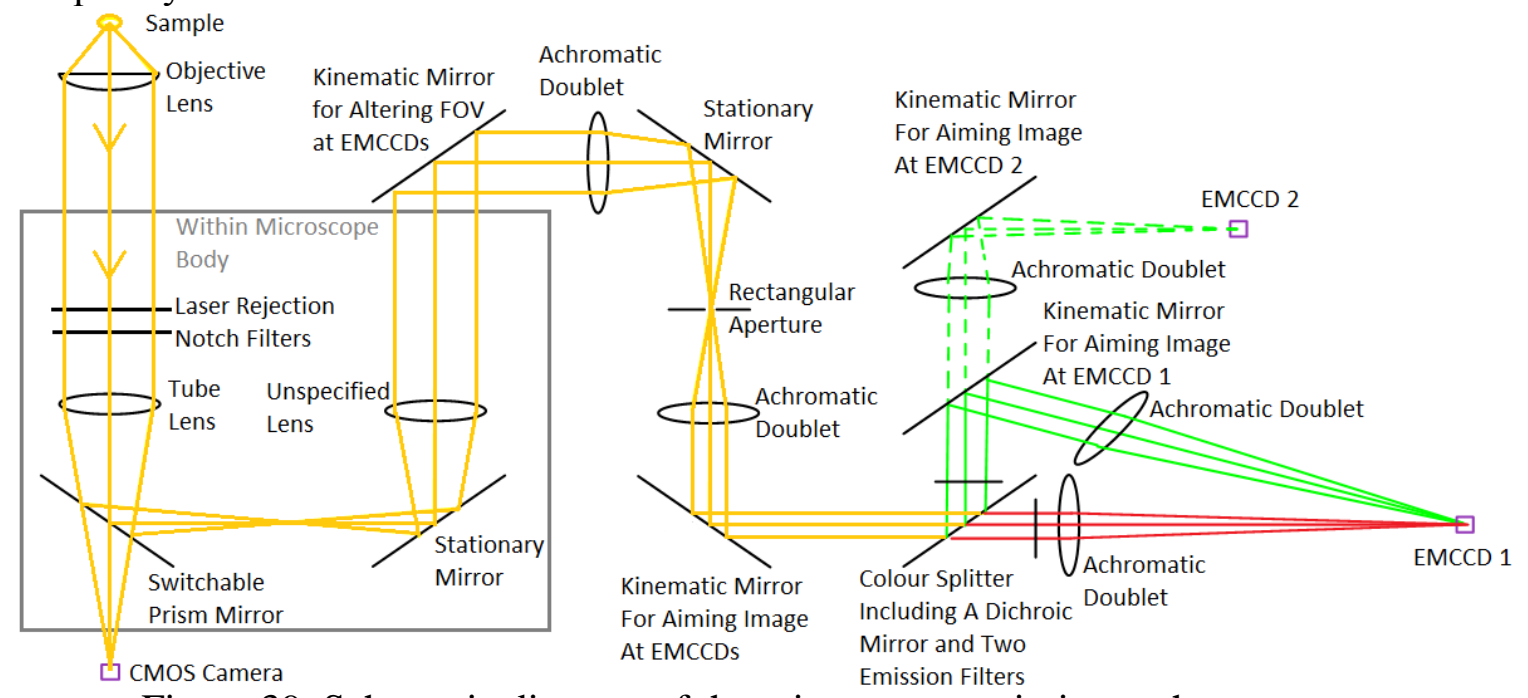

Figure 39 Schematic diagram of the microscope emission path.

The emission path was designed to enable control over the region of the FOV that would be imaged by stationary EMCCD cameras. A kinematic mirror mount with a broadband dielectric mirror was positioned above the port. The beam was reflected through an achromatic doublet that formed an intermediate image plane in the position of 
a rectangular aperture. The aperture was constructed by binding an orthogonal pair of micrometer adjustable slits, enabling variable dimensions. A mount was constructed for the aperture that enabled translation in $x-y-z$ and rotation around the optical axis. The position of the aperture along the optical axis was adjusted coarsely by looking at the intermediate image on its surface, and finely adjusted when looking at the images recorded by an EMCCD. The aperture acted as a field stop that reduced the spatial extent of the image so that multiple channel images could be projected onto adjacent portions of an EMCCD sensor.

The rectangular profiled beam that propagated was aimed at the EMCCDs through the remaining optical components with a second kinematically mounted mirror. The remaining components depended on the type of imaging being performed. The beam interacted with a colour splitting unit that reflected and transmitted high and low frequency bands respectively. The filters would be optimised for the colour splitting of each specific fluorophore combination. 


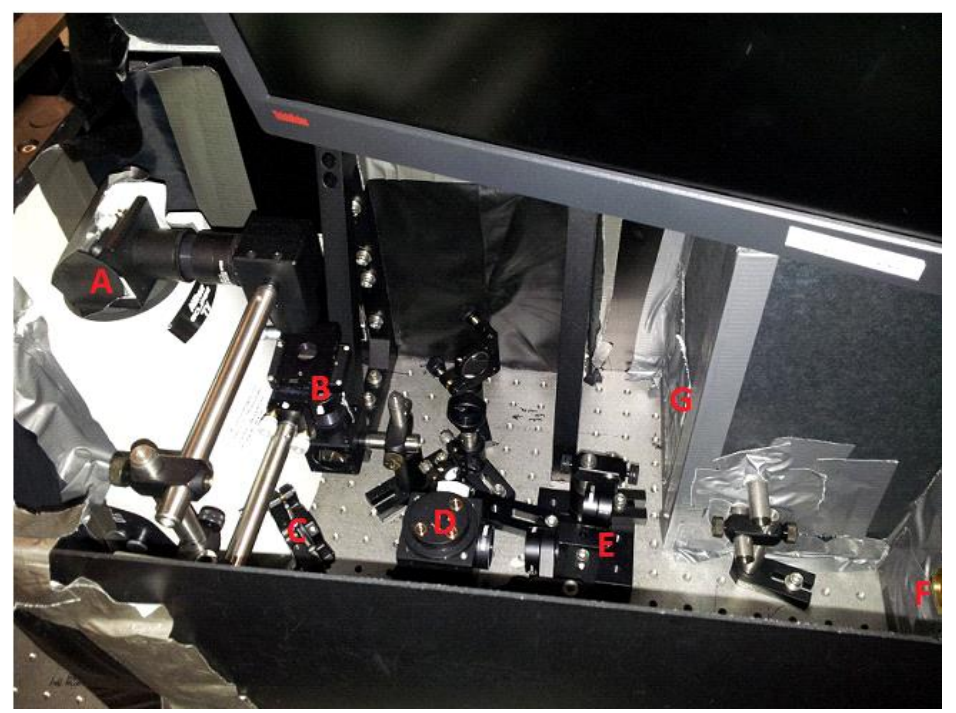

Figure 40 A) Mirror for selecting region of field of view for imaging. B) Variable rectangular aperture. C) Mirror for aiming of image at EMCCDs. D) Colour splitter unit. E) Switchable imaging lens unit on magnetic mount. F) EMCCD 1. G) EMCCD 2.

Magnetically mounted lens modules could be swapped to aim either two images at EMCCD1 or one image at EMCCD1 and one at EMCCD2. The cameras were mounted in a way such that they could be switched in an out with reproducibility on the order of $1 \mathrm{~mm}$ in each spatial dimension. Total containment of the EMCCDs led to their overheating as indicated by whining alarm sounds. The overheating dramatically reduced the sensitivity of the cameras due to the increase in thermal noise. Various approaches to solving the problem were considered including use of an extra fan and water cooling. Ultimately a simple solution was developed based on the insight that only the camera aperture needed to be contained in the light-proof box. Accordingly, apertures were cut in back foam board and placed flush against the camera apertures forming internal walls. The foam board above the cameras was then cut away, allowing the circulation of hot air from the camera with air from the air-conditioned laboratory. 


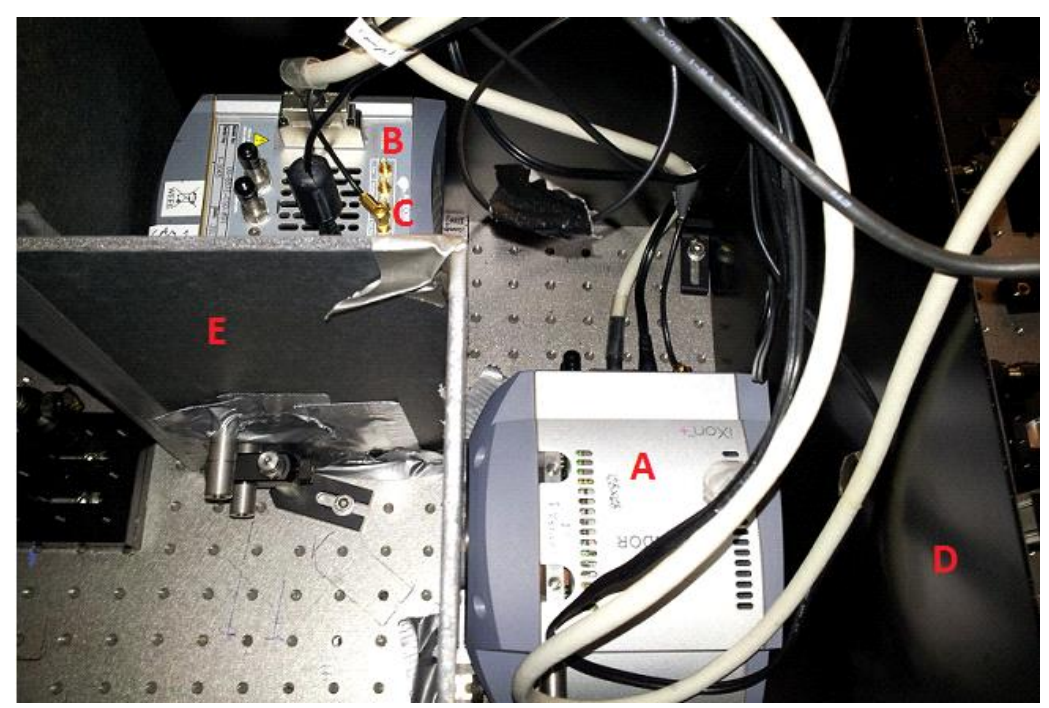

Figure 41 A) EMCCD in position 1. B) EMCCD in position 2. C) Cable relaying external triggering signal. D) Anodized aluminium internal wall to shield emission path from high intensity laser illumination. E) Foam-boar internal wall for cooling of EMCCDs.

\subsubsection{Excitation Path Development}

The microscope was initially designed for use with a supercontinuum laser. While visible bands from the supercontinuum spectrum would be used in fluorescence excitation, the high level of IR radiation posed a safety risk. To reduce the safety risk a box was constructed from anodized aluminium (Figure 42) containing suitable optics to remove the near infrared component of the spectrum. It was also designed to have a possible configuration whereby the near infrared could be channelled for use in sample illumination. An infrared viewer was used periodically to ascertain that IR radiation was not escaping the box. 


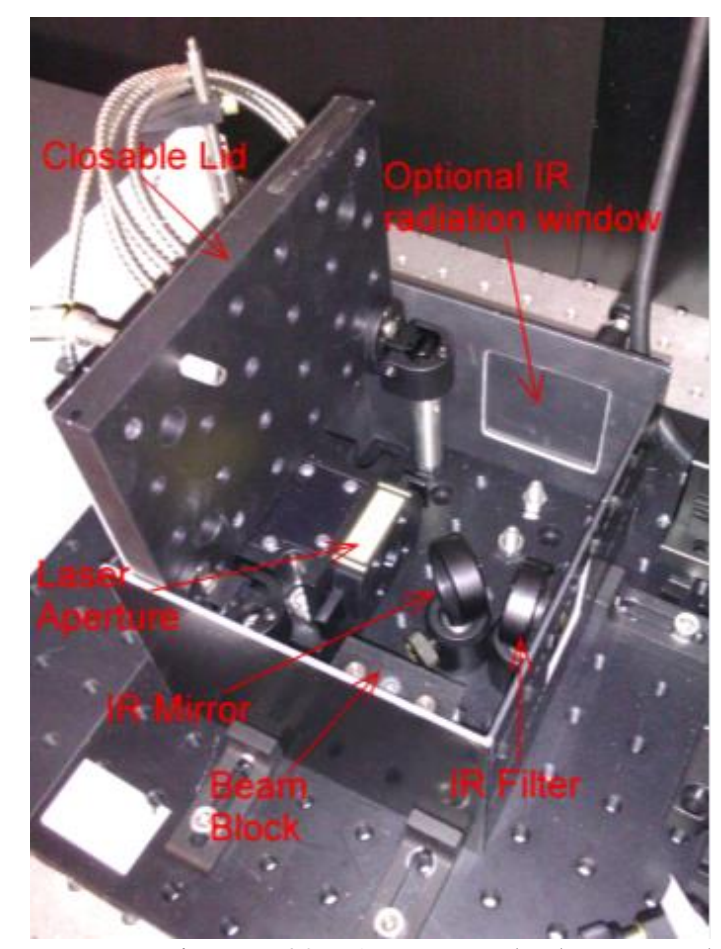

Figure 42 Annotated photograph of laser safety box built to remove IR band of the supercontinuum laser spectrum.

It was desired to have flexibility in the size of sample illumination to adjust for future sample types on the order of $1 \mu \mathrm{m}$ to $100 \mu \mathrm{m}$. Given an approximately Gaussian intensity profile, it would be necessary to produce a field large enough that there would be little change in intensity across the cell, but not so large as to preclude adequate excitation intensities. Accordingly, beam profiles were initially designed to have a standard deviation approximating the longest dimension of each specimen. Many changes were indeed required in the optics controlling field diameter during the life of the microscope. Initially experiments were planned with E. coli cells measuring approximately $5 \mu \mathrm{m}$ in length, but later experiments were carried out on human epithelial cells, measuring approximately $15 \mu \mathrm{m}$ in diameter. In practice it was found that a larger illumination field was preferable in order to reduce the change in intensity across the 
specimen. Ultimately it would be possible to select from two expansion options each path by the replacement of specific lenses.

To solve the problem of positioning all excitation path components within the limited available space the excitation path was modelled in three dimensions in Zemax software (Figure 43). This concurrently enabled verification that the necessary beam trajectories could be achieved with the range of motion achievable with each kinematic component. Components were modelled from catalogue product specifications. Since precise information on the supercontinuum laser spectrum was unavailable, measurements were taken with a digital spectrophotometer. The colours of rays in each channel indicate the spectral components resulting from the chosen dichroic mirrors.

A solution to fitting the multiple paths into the space was found in the vertical stacking paths and the use of a "cage system" to mount the elevated optical elements. Vertical stacking of paths is unusual, partially due to the associated safety risk of high power vertical beams. To address this issue, custom anodize aluminium shields were built and mounted to the kinematic mirrors as extra barriers to vertical beam transmission. 


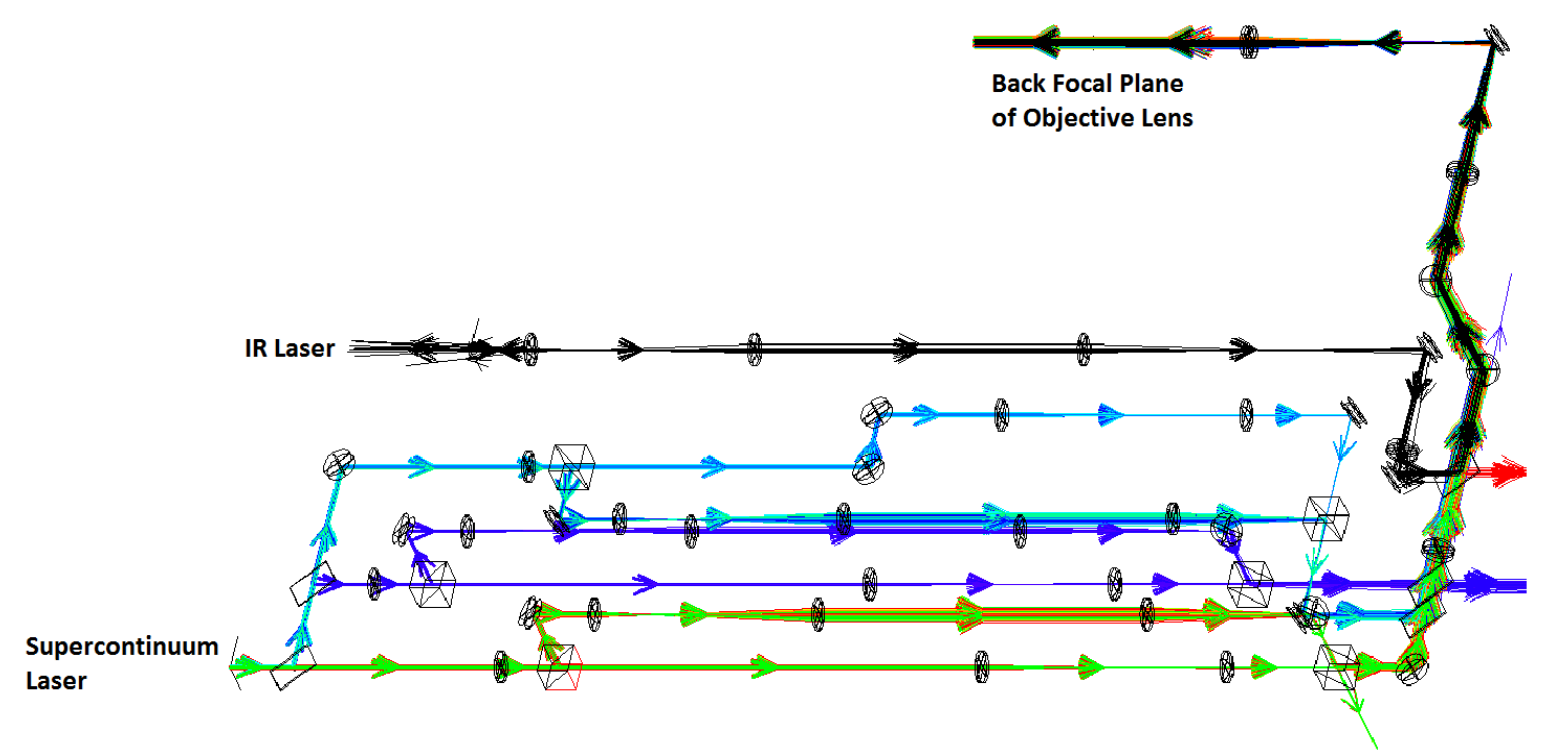

Figure 43 Excitation path rendered with ray tracing software.

An unpredicted event was the breakdown of the supercontinuum source. Due to time constraints it was it was decided that two available monochromatic sources should be integrated, rather than waiting an unknown time for the repair. These $473 \mathrm{~nm}$ and $561 \mathrm{~nm}$ lasers had originated from a different microscope and been mounted in modules to enabled beam expansion to match their differing beam widths, and combine with a dichroic mirror. A periscope was constructed to align the combined laser beam with the pre-existing excitation path. 


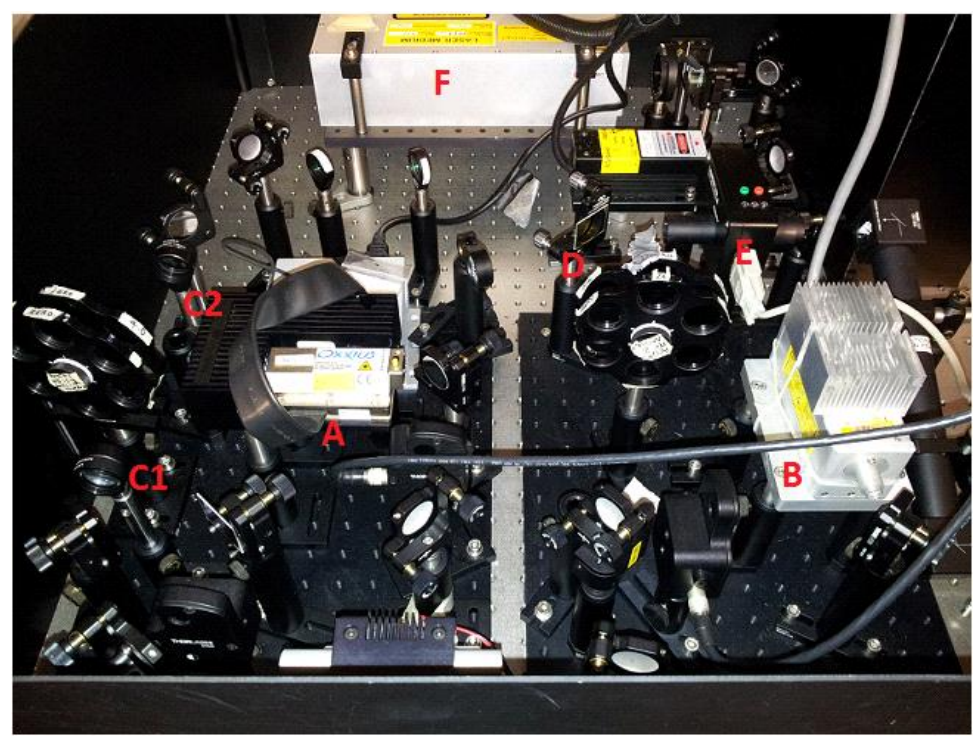

Figure 44 Replacement Lasers. Continuous wave TEM00 laser excitation sources for wavelengths $473 \mathrm{~nm}$ (B4-40, Elforlight) and 561nm (SLIM-561 $200 \mathrm{~mW}$, Oxxius).

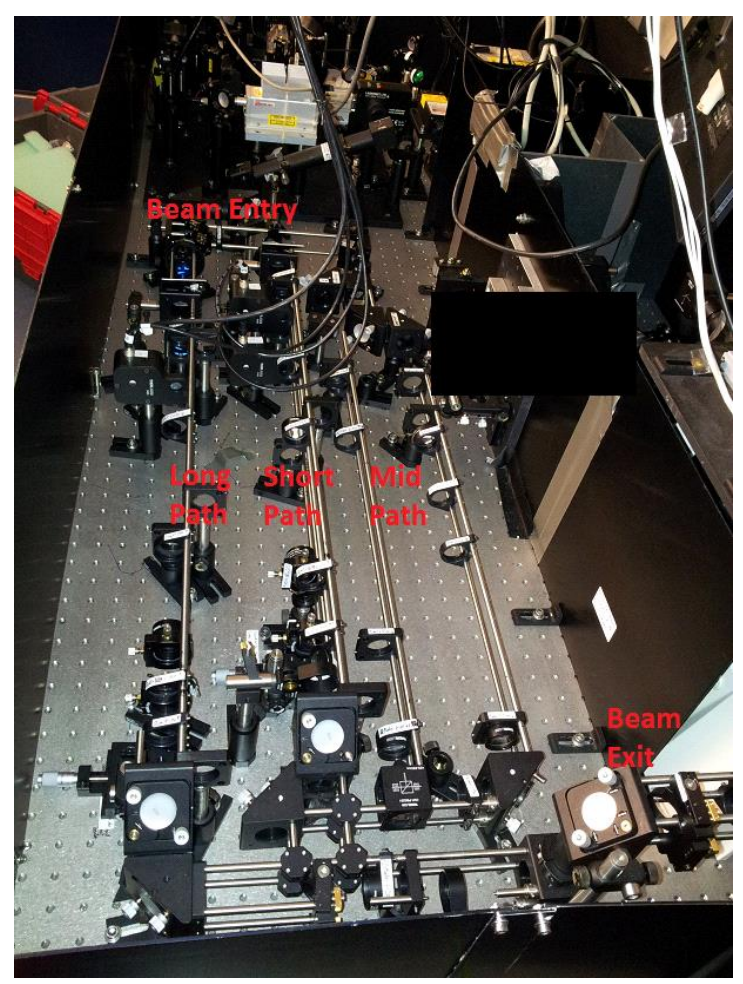

Figure 45 View of the excitation path. "Long", "short" and "mid" refer to the relative wavelengths in each channel. 


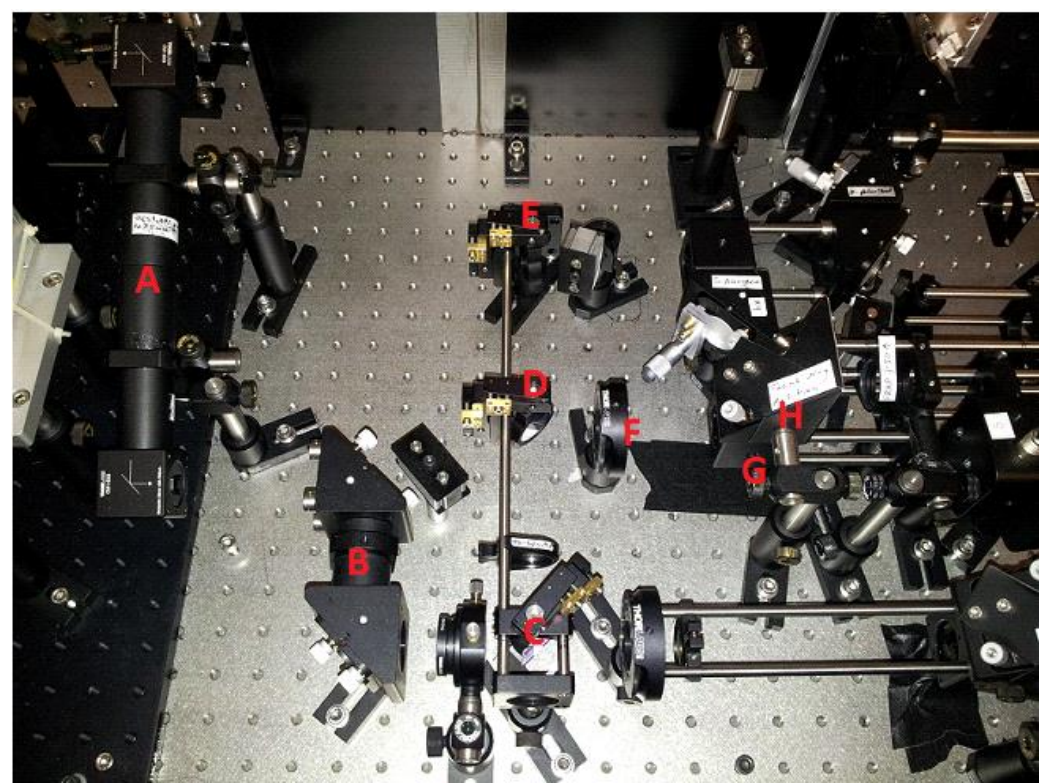

Figure 46 Illumination path set up for use of two monochromatic sources. A) Periscope for lowering beam level of monochromatic beams. B) Kinematic periscope for precise alignment. C) Dichroic mirror transmitting the $561 \mathrm{~nm}$ light through the "long path". D) Mirror reflecting the 473nm light through the "short path". E) Location of gimbal mounts for use in three colour excitation. F) A half-wave plate for controlling the polarisation state of the beam and hence the proportions reflected and transmitted by the Polarising beam splitter cube. G) The polarising beam splitter cube, positioned to transmit a beam for collimated sample illumination and reflect a beam for confocal sample illumination. H) A kinematic mirror for control of the position in the FOV of the confocal volume.

The microscope was designed to be reconfigurable for alternate illumination schemes through the switching of splitter modules. The module used for splitting and steering of $473 \mathrm{~nm}$ and $561 \mathrm{~nm}$ light is presented below in Figure 47. 


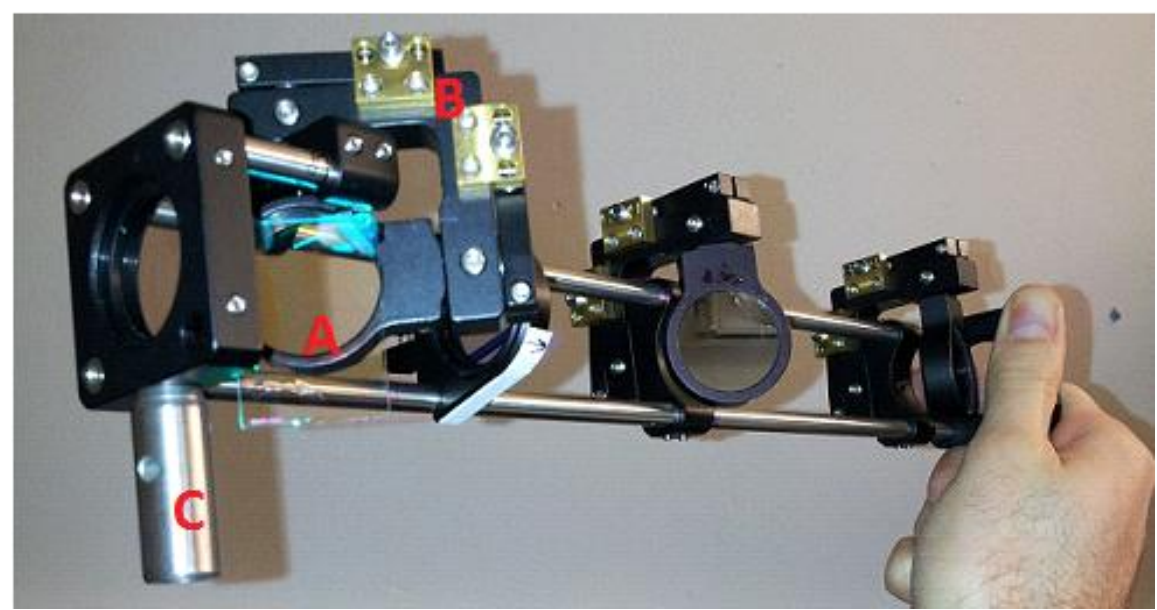

Figure 47 Swappable illumination splitting module used for splitting and steering of $473 \mathrm{~nm}$ and $561 \mathrm{~nm}$ light. A) A dichroic mirror secured with a temporary silicone adhesive. B) Optical elements are positioned and oriented with gimbal mounts. C) The module is constrained and secured in place with posts at each end that slot into post mounts on the optical table.

To reproducibly control the illumination at the sample, kinematic mounts were modified to be controlled with micrometers. This required some machining, but was vastly more affordable than using electronic actuators. Two kinematic mirror mounts modified for micrometer control are visible in Figure 48. This path was laid out flat rather than stacked, to ease reconfiguration. The polarisation splitting module could be replaced reproducibly with an acousto-optical modulator for attenuation of a single unsplit beam. The components for recombination of beams and the interface with the Nikon body are presented in Figure 49 and Figure 50 respectively. 


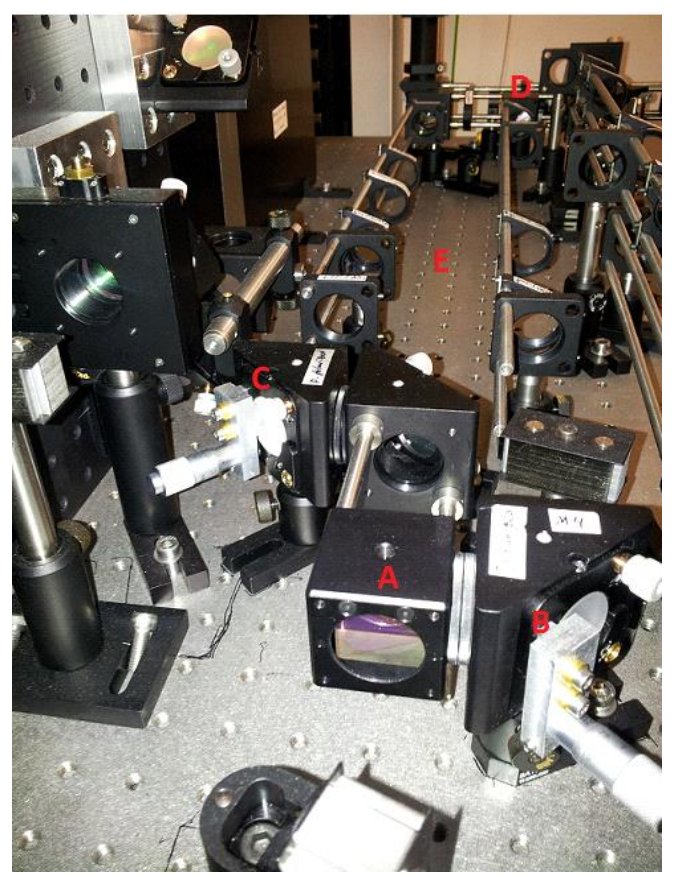

Figure 48 A) Polarising beam splitter cube in "mid path" beam splitting and steering module. B) Control of S-polarised beam. C) Control of P-polarised beam. Kinematic mirror mounts modified for micrometer control of either the collimated angle of incidence or the confocal lateral position at the sample plane. E) Vacant space for unsplit light path.

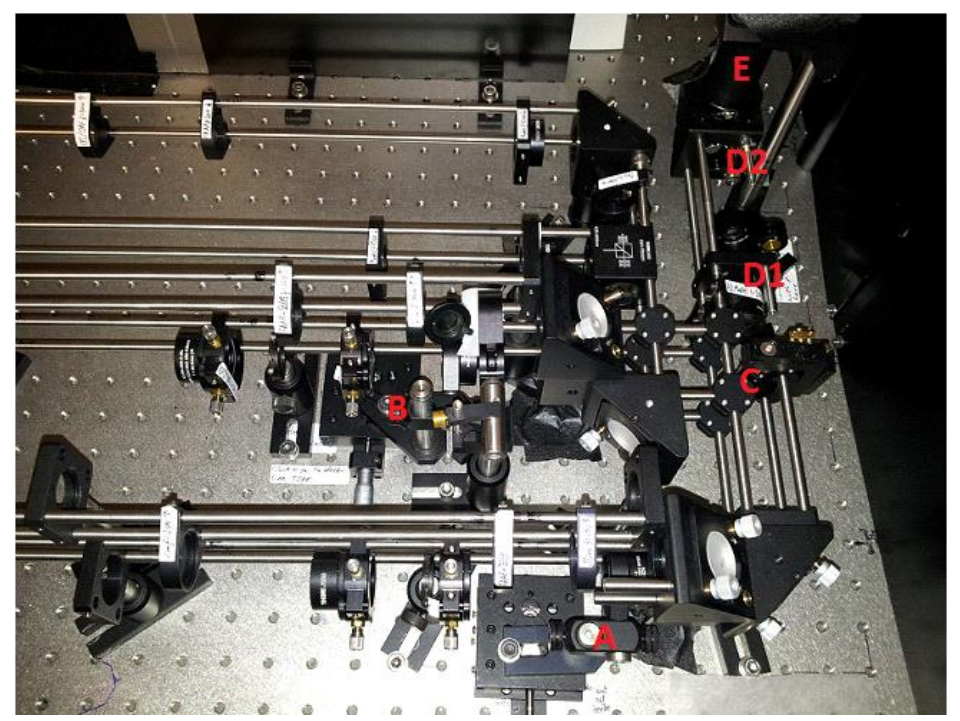

Figure 49 Steering and recombination of the separated colour channels prior to entry into the microscope body. A) Long path Micrometer actuated stage for lateral 
translation of lens controlling angle of incidence of collimated beam illumination. B) Long path Micrometer actuated stage for lateral translation of lens controlling angle of incidence of collimated beam illumination. C) Dichroic mirror on Gimbal mount for recombining beams. D1) Position of first pinhole for beam co-alignment. D2) Position of Second pinhole for beam co-alignment. E) Periscope for alignment of combined beam with the optical axis of the objective lens.

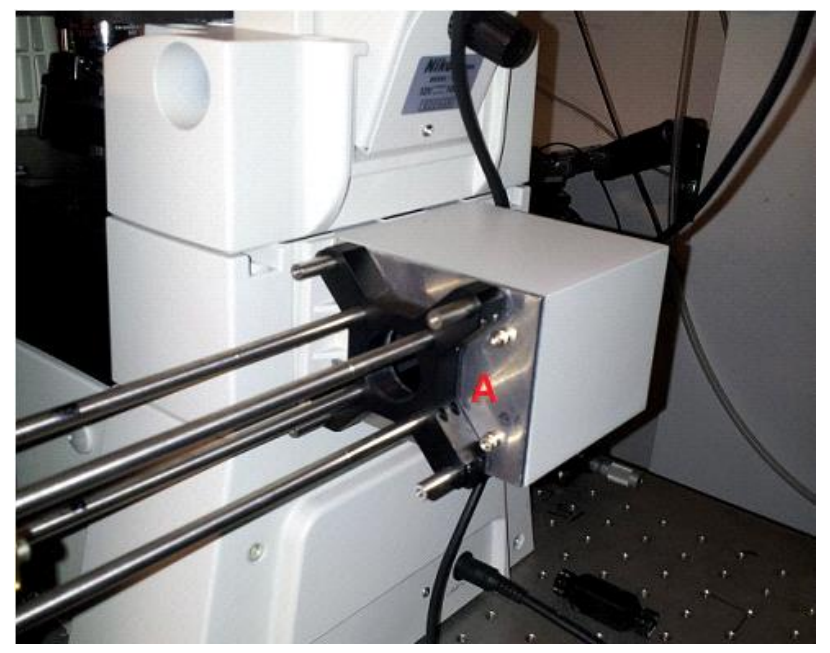

Figure 50 A bracket was built for robust and reproducible connection of the cage system to the Nikon microscope body.

\subsubsection{Alignment and Calibration}

In building a relatively complicated optical instrument much care was necessary in the precise positioning and alignment of each component. Initially the illumination path lenses needed spacing. An attenuated laser beam was shone through two consecutive lenses at a time and the spacing modified while observing the beam profile striking the laboratory wall a maximal distance away. The lens spacing would be locked when the beam width at the wall equalled the width from the source, making sure that 
there was no focal point between the second lens and the wall. This process was repeated for each consecutive lens pair, starting from the objective lens and moving upstream towards the source. All but the two lenses being spaced were removed.

The illumination paths were then precisely steered into position. Primarily each path was steered so as to pass through two pin holes placed prior to the combined channel periscope. Once all beams had been individually aligned to pass through the pinholes, the combined path was aligned to pass along the optical axis of the objective lens. Prior to the use of image sensors, the two reference points for this alignment were the centroid of the objective lens port and a point marked on the ceiling vertically above this point. To mark the intersection of the objective lens optical axis on the ceiling it was first necessary to confirm with a spirit level that the optical table was horizontal. Next a point directly above was marked on the ceiling with a tool made from a long stick with a pen attached to the end and a plum line dangling from the pen nib. All future alignment of the illumination path would use the ceiling reference point and the centre of the field of view as imaged in the sensor located on the optical axis at the side port intermediate image plane.

Brightfield illumination was used to align the emission beam and a recognisable object was centred in the field of view of the side port sensor. Then the prism mirror below the objective was rotated to send rays towards the EMCCD sensors and the kinematic mirror was oriented to direct the beam centrally through the downstream components. The rectangular aperture was closed so that the object image was focussed on its surface in a central position. Next the aperture was opened partially and the beam was directed sequentially at each EMCCD sensor with the respective kinematic mirror. 
The brightfield illumination intensity was then reduced and images were taken with each EMCCD to achieve precise aiming of the beams and the correct rectangular aperture size. Finally all emission path imaging lenses were co-focussed using $0.2 \mu \mathrm{m}$ latex beads. The precise co-focussing of the colour channels was vital for effective imaging and analysis.

\subsubsection{Imaging}

Over the course of experimentation numerous modifications were made to the form of flow cell used to house the samples. Initially flow cells were constructed by sandwiching two tracks of viscous grease between a slide and coverslip. The use of grease was replaced by the use of double sided sticky tape for reproducibility of air gap thickness. To enable multiple sample types per flow cell, multiple lanes were made in each flow cell.

Initial fluorescence imaging revealed fluorescent contaminants on coverslips fresh from their packaging. This contamination was resistant to cleaning with multiple detergents and solvents, and ultrasonication. The contaminants were totally removed by the process of plasma cleaning and compact plasma cleaning system was obtained for the laboratory.

Poly-L-lysine $(0.1 \%$ in water added to flow cell, then washed through) was initially used to immobilise living cells to the coverslip surface but was replaced by the

pressure exerted by an agarose gel. Use of agarose gel resulted in almost all cells flat to the coverslip enabling effective membrane imaging. To image epithelial cells pre- 
fabricated flow cells with six channels were acquired that were relatively easy to populate with the samples.

The first image sensor used at the microscope side port was a Watec CCD. Using a graticule (Figure 51), a slide marked with ten micron increments, the field of view was measured as 64 microns across. Given an array width of 6.45 millimetres the magnification was calculated as $100 \mathrm{X}$, in agreement with the expected magnification calculated from the focal length of the Tube lens divided by the focal length of objective lens $(100 \mathrm{~mm} / 2 \mathrm{~mm})$.

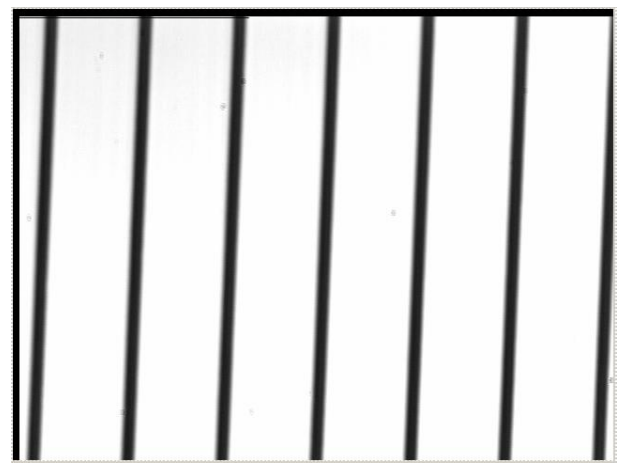

Figure $5110 \mu \mathrm{m}$ increments on a graticule imaged in brightfield with a CCD (Watec) at the side port.

Early images exhibiting fluorescence illumination are presented below. To view the extent of the fluorescence illumination fields for both green and red emitting fluorophores, a sample was prepared with a dense distribution of $100 \mathrm{~nm}$ diameter orange fluorescent beads (Carboxylate-Modified Microspheres, Life Technologies). To immobilise the beads on coverslip surface, the flow cell was first filled with a solution of poly-L-lysine $(0.1 \%$ in water), then rinsed through with water prior to addition of the bead suspension $(0.0004 \% \mathrm{w} / \mathrm{v}$ in water $)$. 


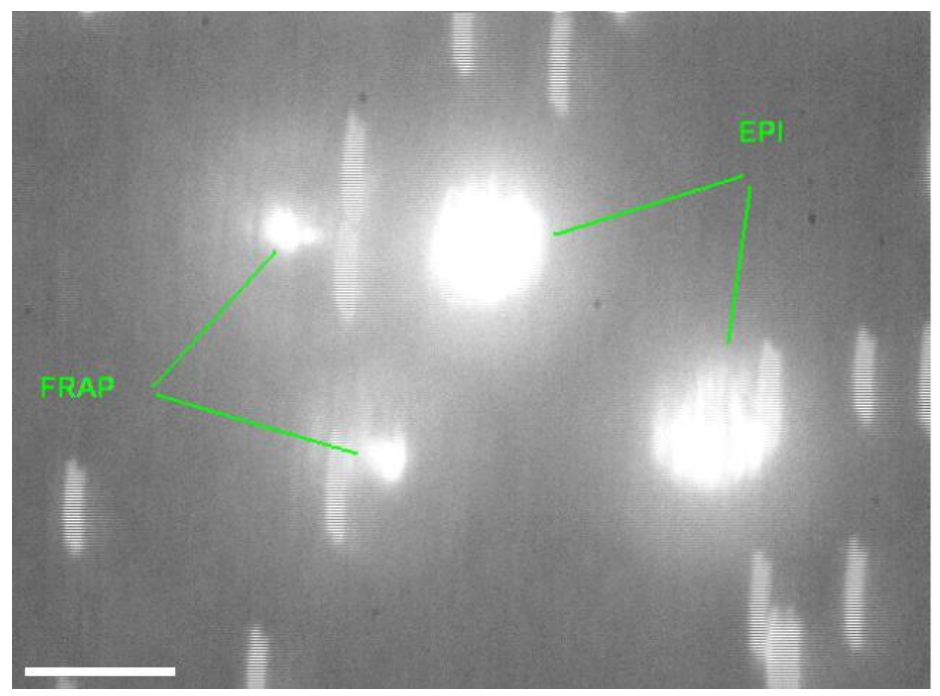

Figure 52 Minimal confocal and epifluorescence with each colour channel and dim brightfield illumination of a sample being translated laterally during the exposure. The sample contains densely distributed fluorescent beads and $1 \mu$ latex beads that can be identified through their elongation. $10 \mu \mathrm{m}$ scale bar.

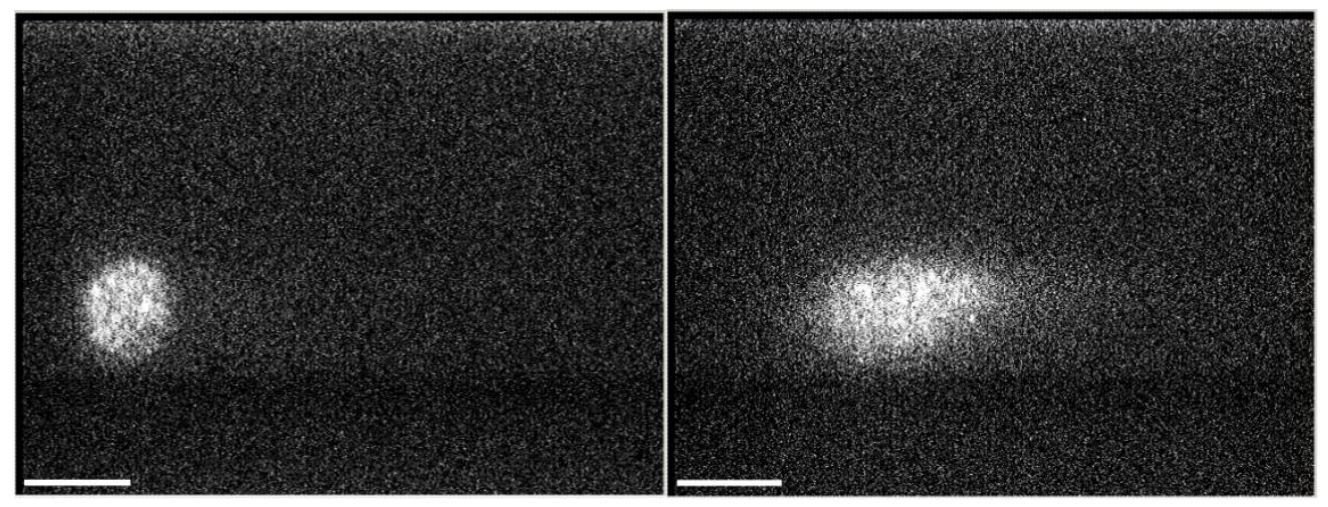

Figure 53 Epifluorescence left and the same beam steered into TIRF illumination at the critical angle. $10 \mu \mathrm{m}$ scale bar.

Images are presented to illustrate the TIRF illumination of Green and Red fluorescent $200 \mathrm{~nm}$ diameter latex beads and 1micron latex beads seen through brightfield. 

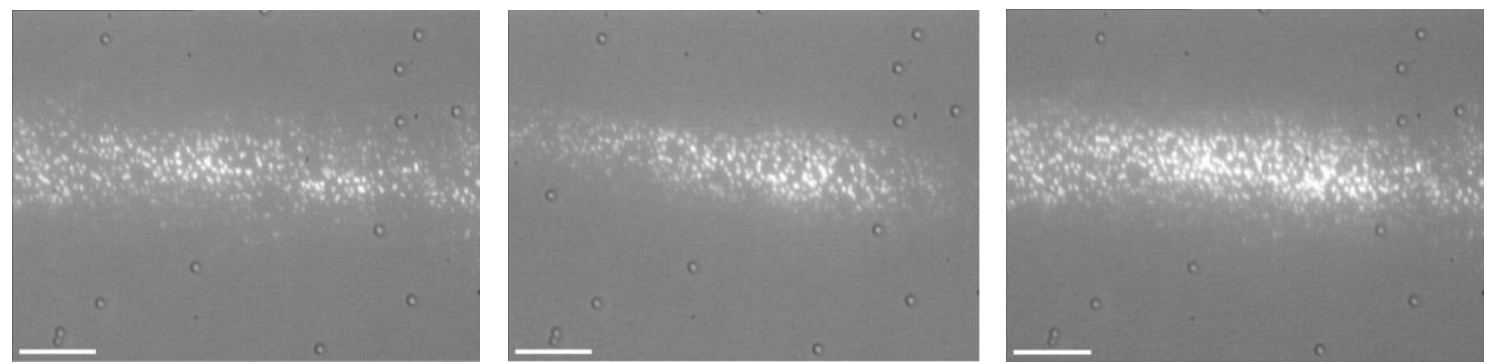

Figure 54 Tirf and brightfield illumination of $200 \mathrm{~nm}$ green and red fluorescent beads, and $1 \mu \mathrm{m}$ non-fluorescent beads. TIRF illumination with $473 \mathrm{~nm}$ (left), $561 \mathrm{~nm}$ (centre), $473 \mathrm{~nm}$ and $561 \mathrm{~nm}$ (right). $10 \mu \mathrm{m}$ scale bars.

Upon establishing the EMCCD emission path, images were taken to evaluate the effectiveness of colour splitting and the formation of adjacent images on a sensor. In Figure 55 below green and red images of the same field of view are projected on the $128^{2}$ pixel EMCCD array. Elongation of each fluorophore image is a result of the inclusion of a Wollaston prism in the optical train that had previously been used for differential interference contrast (DIC) imaging.
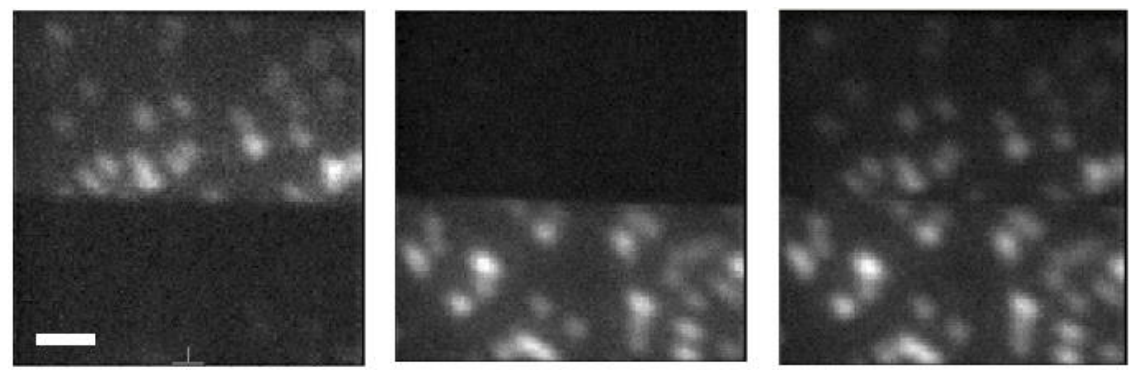

Figure 55 Splitting of TIRF images of $200 \mathrm{~nm}$ green and red fluorescent beads onto separate halves of $128^{2}$ pixel EMCCD. TIRF illumination with $473 \mathrm{~nm}$ (left), 561 $\mathrm{nm}$ (centre), $473 \mathrm{~nm}$ and $561 \mathrm{~nm}$ (right). Scale bar $1.5 \mu \mathrm{m}$. 


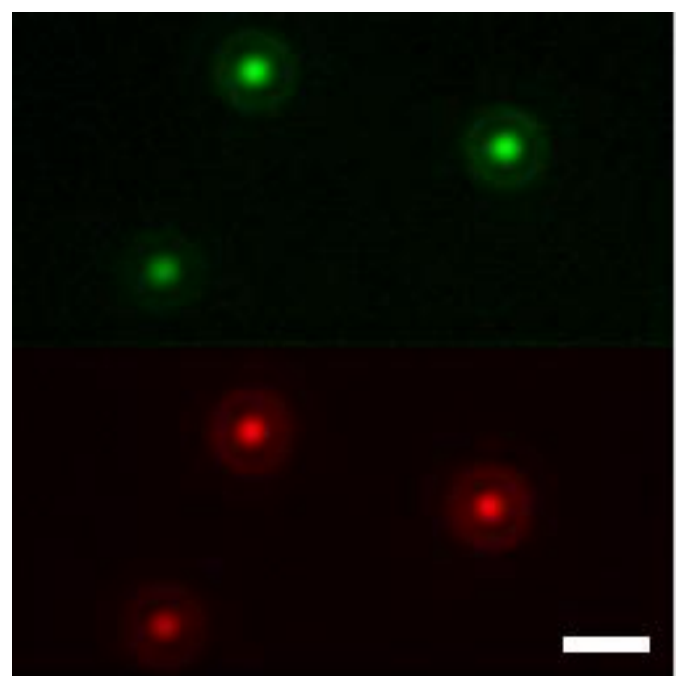

Figure $560.1 \mu \mathrm{m}$ orange beads were imaged to record the PSF and for future comapping between colour channels. False coloured image. Scale bar $1 \mu \mathrm{m}$.

Further to the fluorescence imaging, the ability to exert force through infrared laser tweezers was integrated in collaboration with Dr Llorente Garcia. Using the confocal volume (Figure 57), $1 \mu \mathrm{m}$ diameter latex beads (Life Technologies) were repositioned on the surface of the coverslip surface.

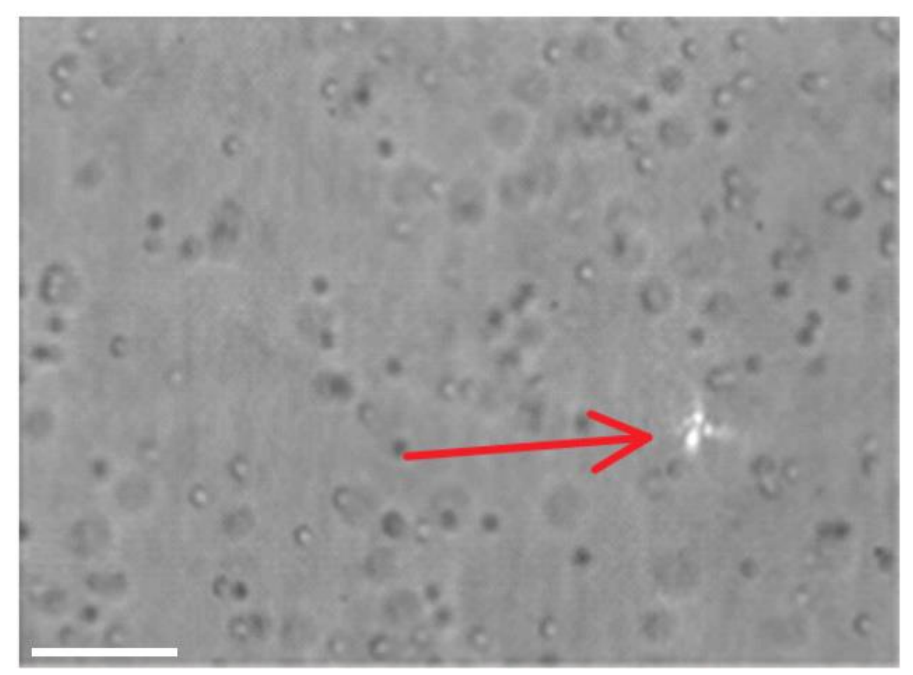

Figure 57 Brightfield image of $1 \mu \mathrm{m}$ latex beads in solution and optical tweezers IR confocal volume indicated with red arrow. $10 \mu \mathrm{m}$ scale bar. 
Numerous steps were found to be necessary in the acquisition of images. The foreseen live-cell experiments would benefit greatly from reducing the number of manual steps. Automation of aspects of the experimentation would improve reproducibility and free up more time for evaluation of experimental progress and data. The LabVIEW programming environment was selected for this task and used to develop event-based, graphical software for control of the microscope. The software was initially constructed to control the instrument with front panels specific to each stage of experimentation and experiment type. Later a streamlined version of the software was written specified for the experiments that were undertaken. The key functionality of the software was the triggering of events in the image acquisition sequence. The software was used to predefine and initiate the timing sequence for the EMCCD acquisition triggering, and the opening and closing of the laser shutters. The EMCCD imaging parameters were initialised through the proprietary Andor "Solis" control and analysis software. 


\subsection{Model-Aided Experimentation}

A view was taken that my experimentation should be engaged with at the systemlevel. This section presents a range of efforts aimed to enhance my experiments through internally consistent computational models. These were based on what was seen to be relevant information, much of which is not typically considered systematically. Quantitative modelling was explored as a means to improve capabilities of the instrument, to enable calculation of new physical values, and to improve the performance of the experimental system (i.e. obtain higher quality data).

\subsubsection{MODEL-INSTRUCTED CONTROL OF ILLUMinATION}

\subsubsection{Prescriptive Polarisation-Based Power Distribution}

As a first example, a relatively simple optical scheme will be used to demonstrate how a model based approach may improve the capabilities of an instrument and reduce the number of necessary components. The combination of a half-wave plate and polarizing beam splitter is commonly used to continuously attenuate a laser beam of constant power. This scheme is also used to vary the relative proportions of power going into two channels (Figure 58). The sum of output powers is constrained to equal the input power (Figure 59). 


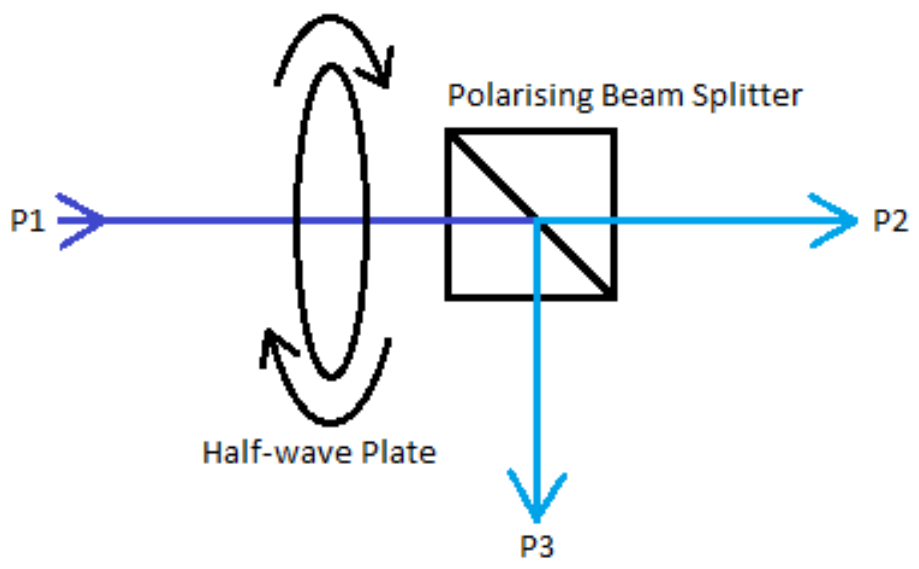

Figure 58 Rotating the half wave-plate changes the ratio of the output powers.

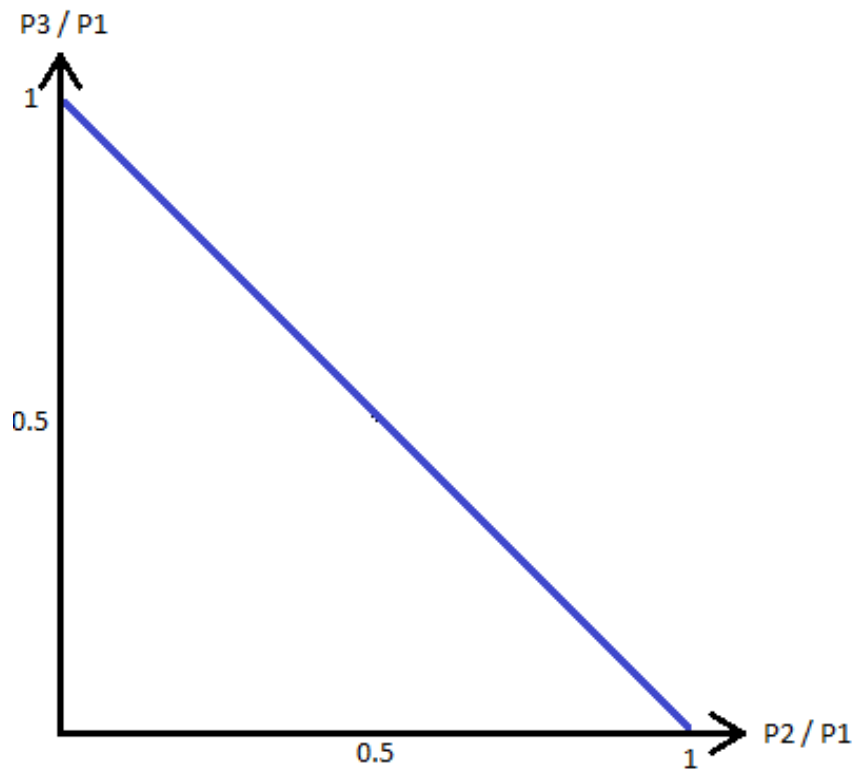

Figure 59 The blue line indicates the possible simultaneous output powers in the scheme of Figure 58.

For independent control of the power in each output path further components are necessary. A straightforward solution involves putting a rotating variable neutral density (ND) filter in each of the split channels (Figure 60). In practice, the half-wave plate could be set to split the beam 50:50, and then independent power control of each split 
channel would be achieved with the respective ND filter. This is a very pragmatic scheme; however it has limitations (Figure 61). To produce a split of 80:10, the half wave-plate would need to be rotated and then both ND filters reset.

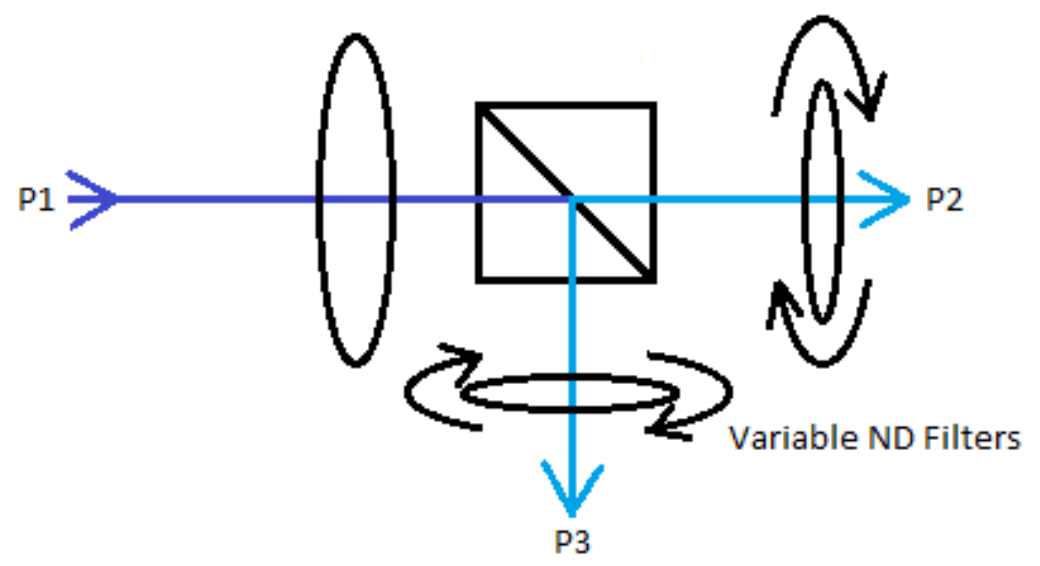

filters.

Figure 60 Independent power control is achieved with variable neutral density

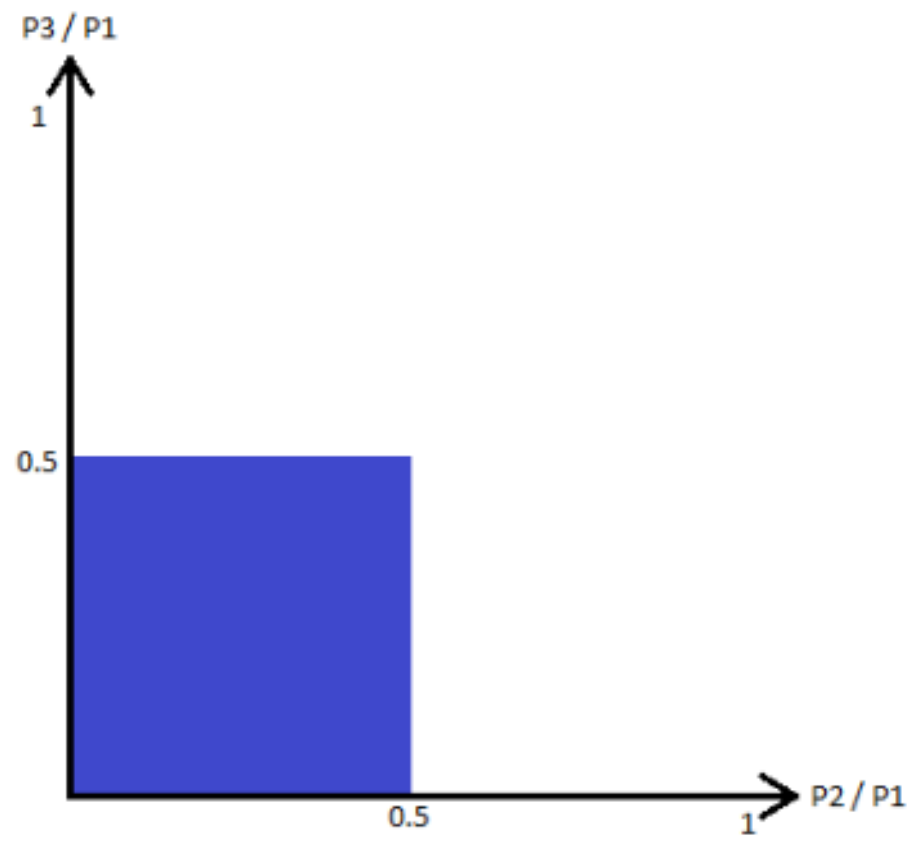

Figure 61 The possible range of powers of the scheme in Figure 60 is limited by the setting of the half-wave plate. 
In order to achieve independent control and the full range of power for each path, two options exist. Either one could measure the power while changing the angles; or the necessary angles could be calculated from the equations that describe transmission through the system. If one uses the second approach, a single ND filter prior to the polarising beam splitter will achieve the same function as two after (Figure 62). One simply need specify the desired powers in order to determine the necessary settings (Figure 63).

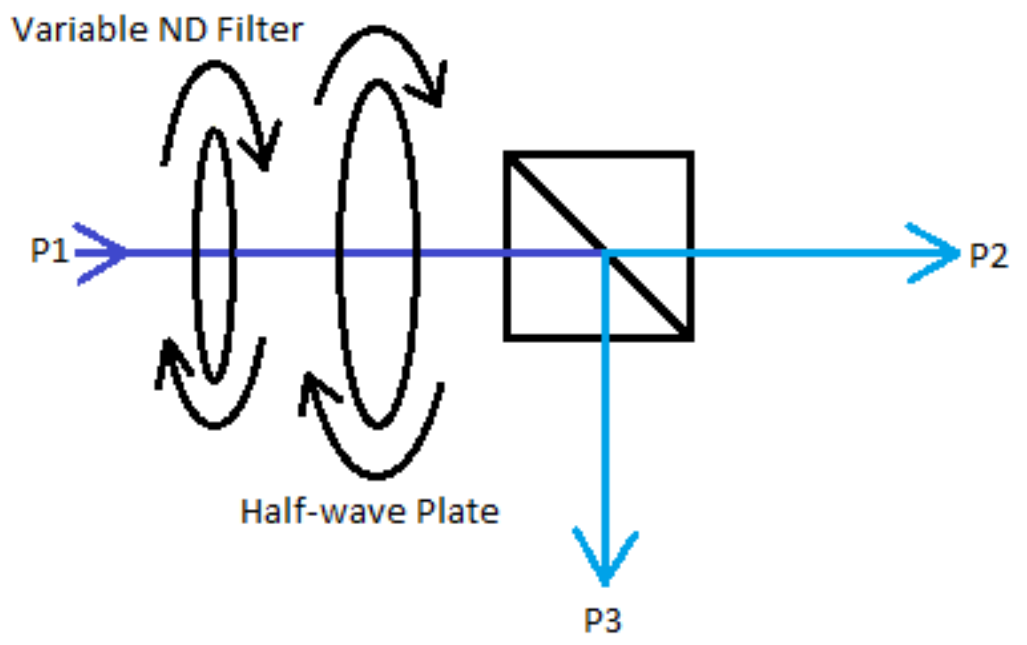

Figure 62 This scheme achieves independent control but relys on prescriptive control from a model. 


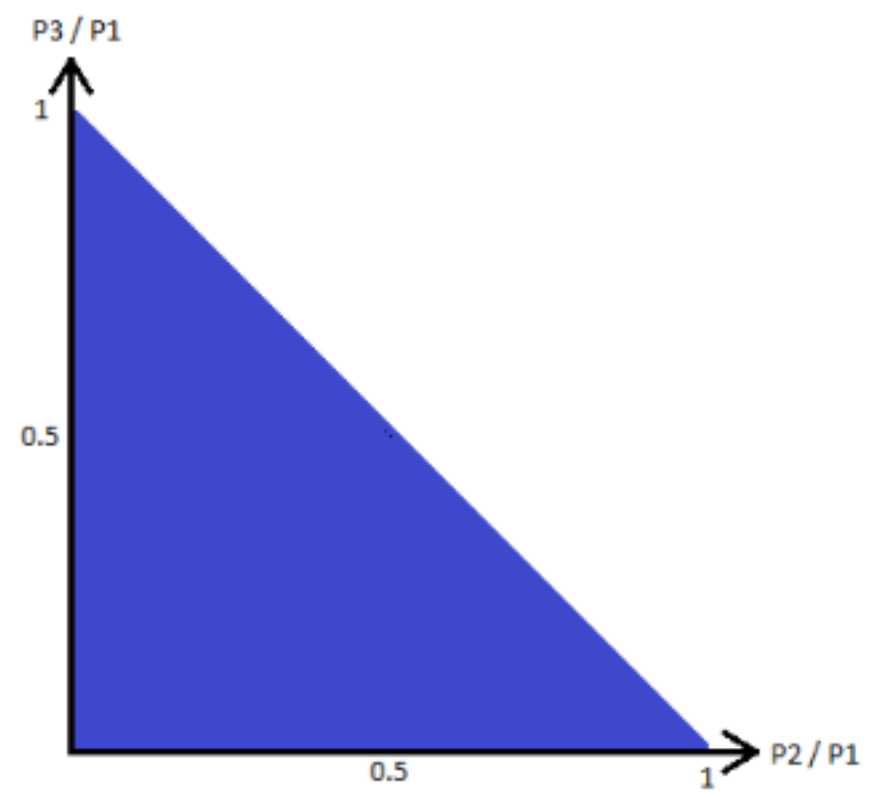

Figure 63 With the scheme in Figure 62, the maximum range of simultaneous output powers can be produced.

\subsubsection{Spectral Band Selection for Two-Colour Excitation}

From inspecting the absorption and emission spectra of two commonly used fluorescent proteins, one can identify approximately optimal wavelengths for excitation (Figure 64). As well as for obtaining a high signal, this choice is important in reducing a number of effects including, photobleaching, phototoxicity, and the artefacts known as crosstalk and bleedthrough. Using monochromatic light leaves the maximal bandwidth for collection of Fluorescence emission. 
Overlapping Absorption And Emission Spectra Of Two Fluorescent Proteins Normalised Relative Absorption And Transmission

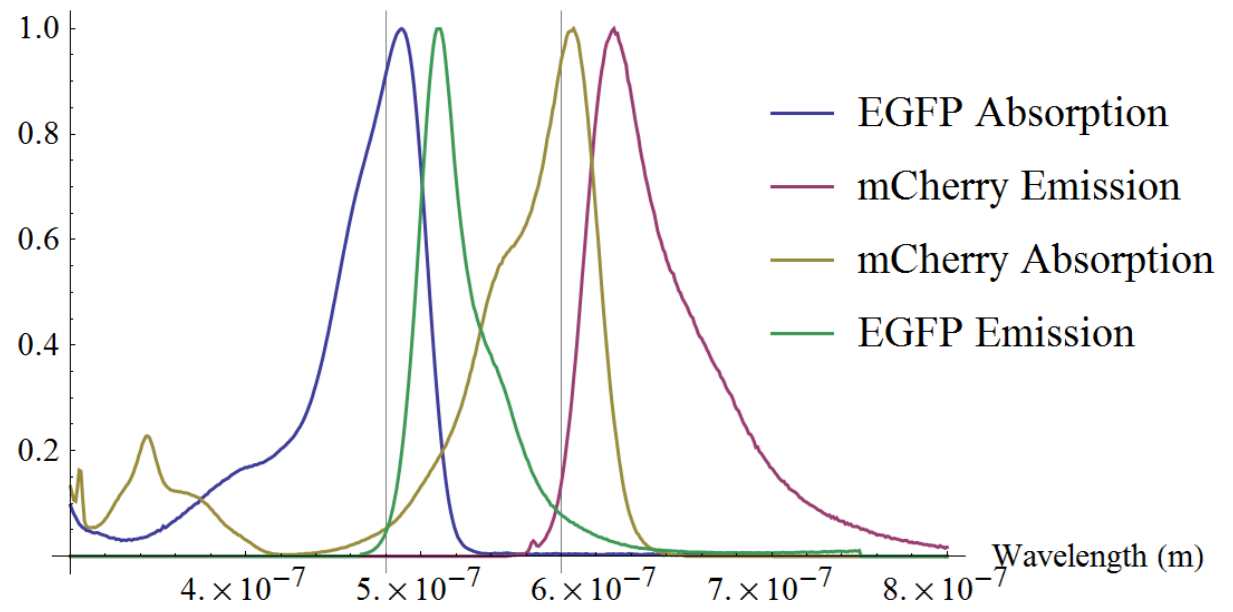

Figure 64 Absorption and emission spectra of EGFP and mCherry. Approximately optimal illumination wavelengths indicated with vertical grey lines. Spectra normalised by height for ease of comparison.

Rather than use multiple lasers for different wavelengths, one might use one supercontinuum source, giving flexibility to select arbitrary bands from the visible range. However, since the total power is spread out over the visible and IR spectra, there is potential for being power limited if using only a thin band from the spectrum. In this scenario the fluorescence emission gained from broadening the band of illumination may outweigh the emission lost from the resulting smaller band for collection of fluorescence.

With the IR component of a supercontinuum beam removed, the remaining spectral component was measured at a series of laser power settings (Figure 65). It is evident that the spectrum changes significantly with increasing power so it was decided that only $100 \%$ should be used for consistency. 


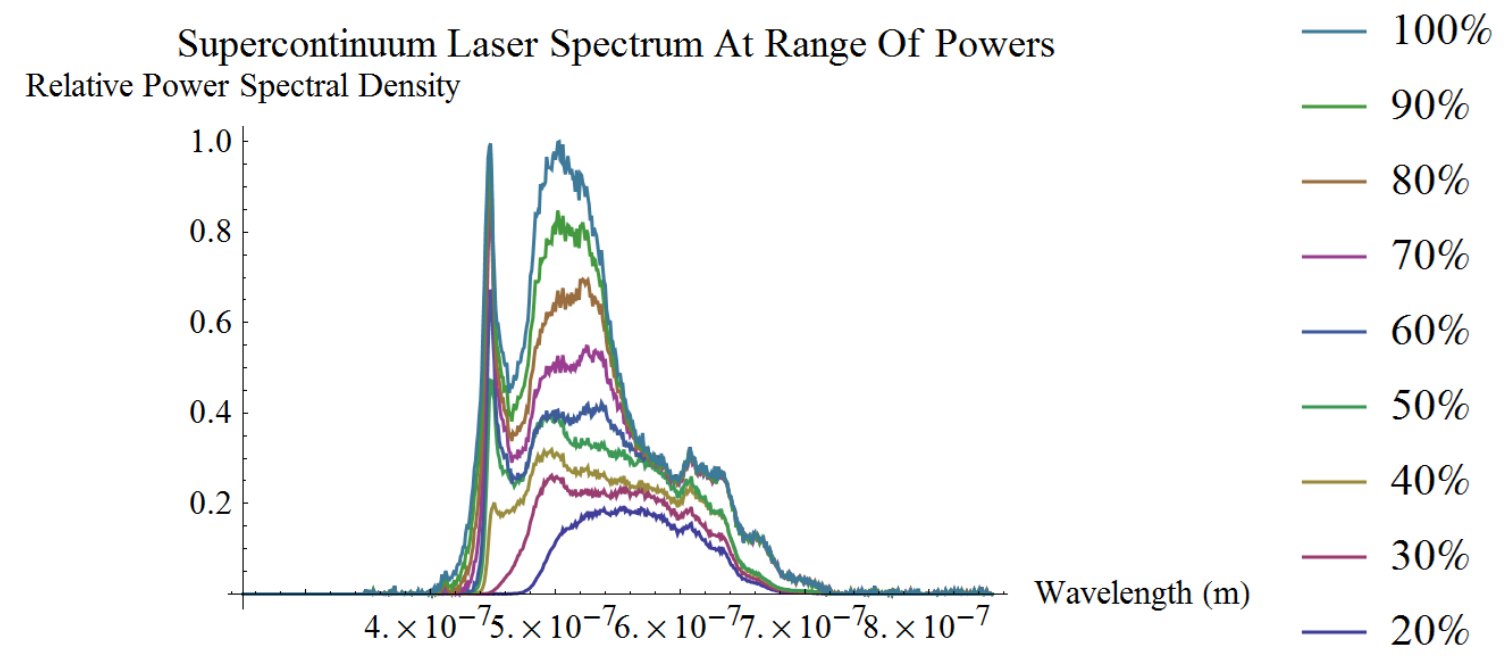

Figure 65 Supercontinuum spectra measured at a series of powers as set by the laser controller.

A simulation model was implemented to investigate how the selected spectral bands could be optimised computationally for different scenarios. The spectral selection method was based on the pattern of dichroic mirrors used in the microscope (Figure 34) where the two bands were selected by setting the cut-off wavelengths of three dichroic mirrors. To identify the cut-off wavelengths giving best performance, an objective function was evaluated at every point in the three-dimensional parameter space and the maximum value identified. The objective calculated the sum of the two emission signals (minus their respective artefact levels) and subtracted the magnitude of their differences. This led to a maximum where the signal in both bands was high.

Several scenarios were investigated. In the first case, both fluorophores were given approximately the same inherent brightness. The optimal illumination bands (Figure 66) and the resulting signal and artefacts are displayed (Figure 67). 
Optimum Excitation Bands for Fluorophores of Equal Brightness Relative Power Spectral Density

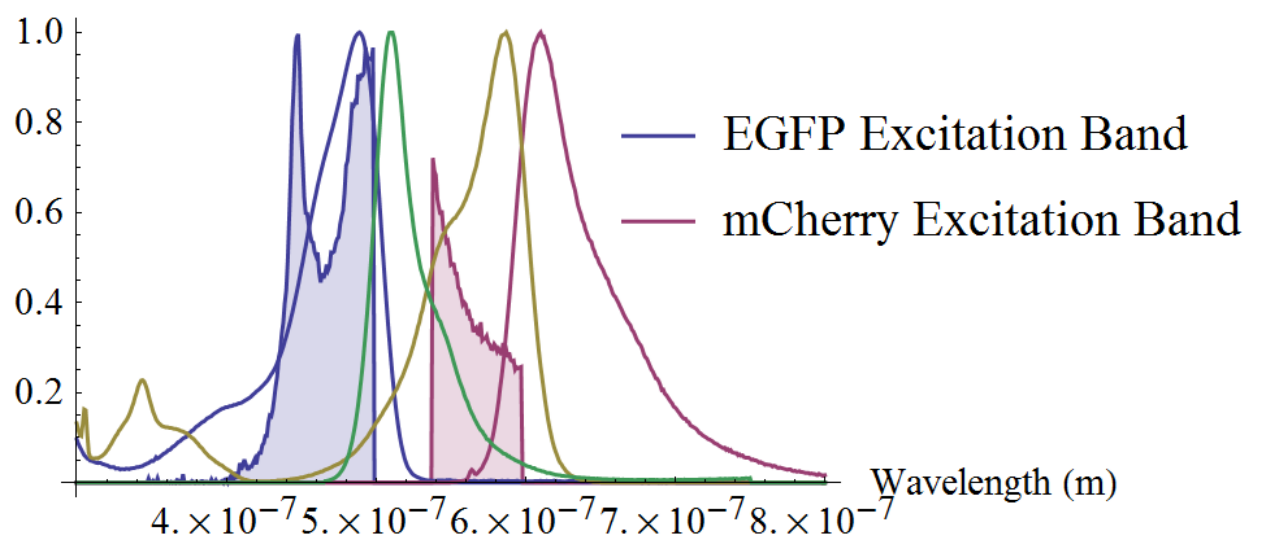

Figure 66 The optimal bands from the supercontinuum spectrum for exciting similarly bright fluorophores.

Signal and Artefact Emission for Fluorophores of Equal Brightness Relative Collected Fluorescence

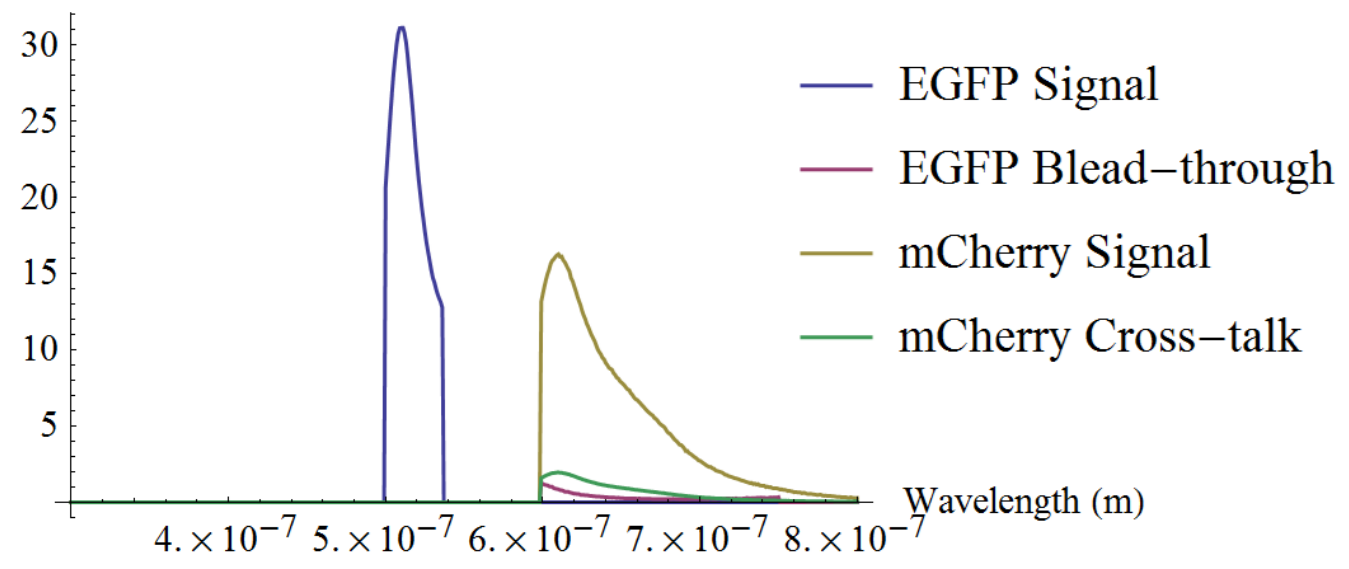

Figure 67 The Fluorescence emission resulting from the illumination in Figure 66. 
In another case, mCherry was given a much lower inherent brightness. It can be seen that this results in a significant change in the optimal illumination bands (Figure 68) and output fluorescence (Figure 69).

Optimum Excitation Bands for EGFP Ten Times Brighter

Relative Power Spectral Density

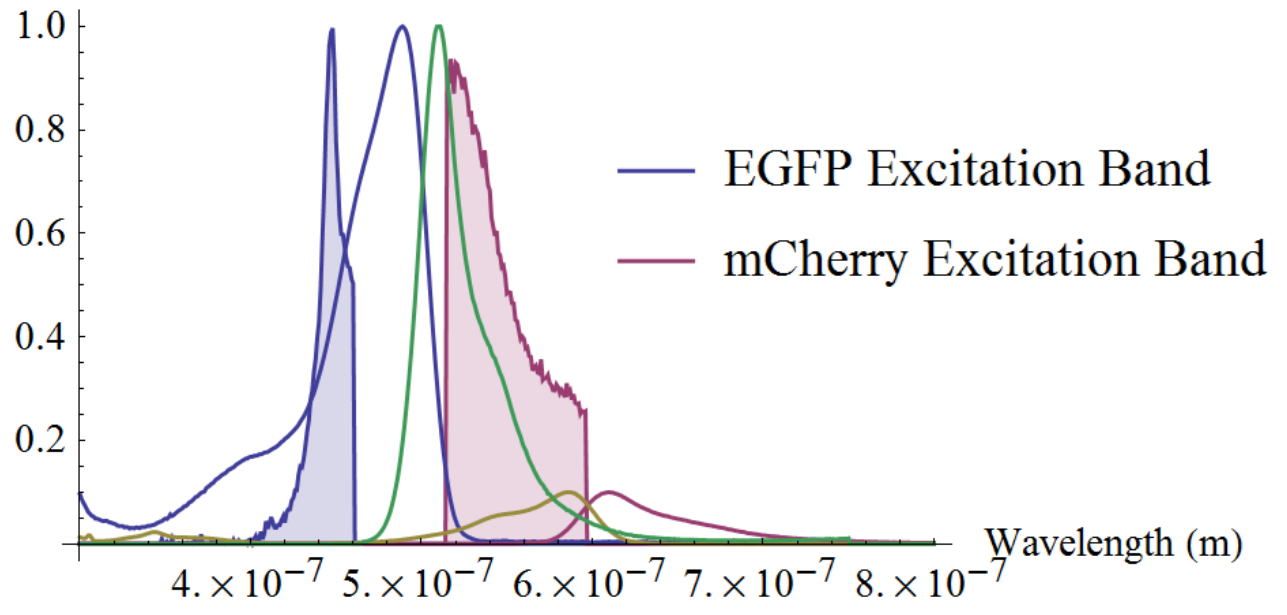

Figure 68 The optimal illumination bands can be seen to change with the relative brightness of the two fluorophores. 
Signal and Artefact Emission for EGFP Ten Times Brighter Relative Collected Fluorescence

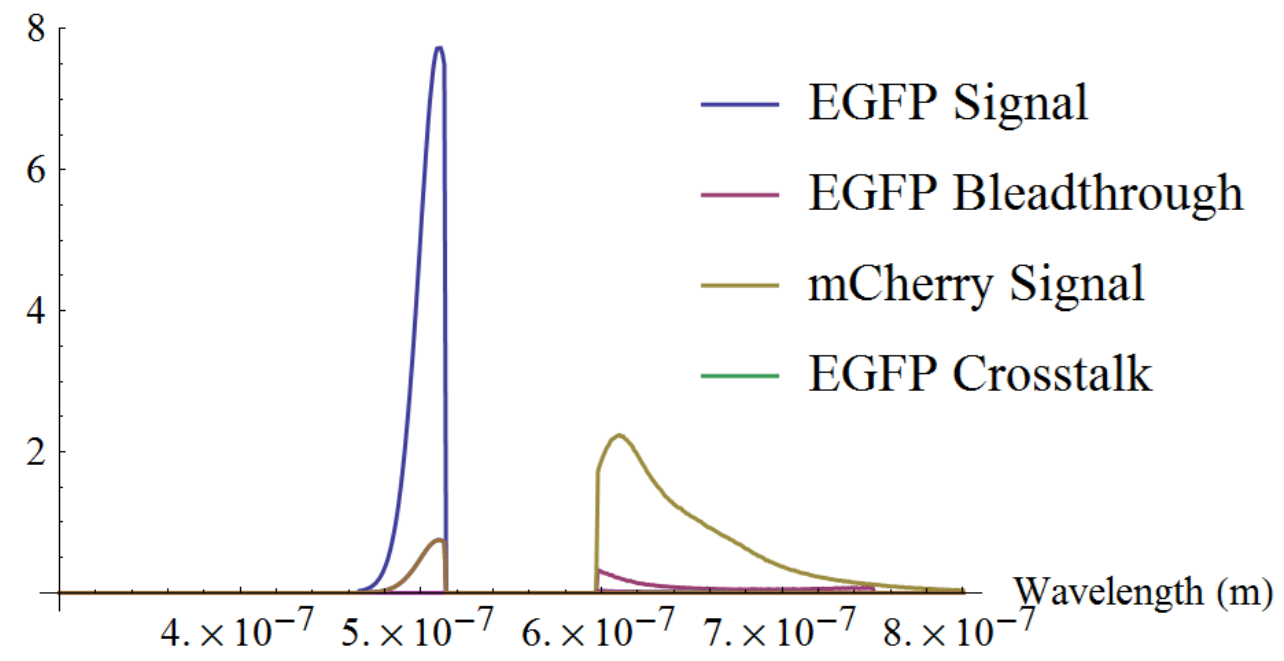
68.

Figure 69 The Fluorescence emission resulting from the illumination in Figure

\subsubsection{Reverse Ray Tracing to Inform Beam Steering}

\subsection{Modelling of the Objective Lens}

The microscope would make use of a Nikon CFI Apo TIRF 100X Oil objective lens. With the minimal available lens design information it was only possible to treat the lens as a black box. However in order to produce an in-silico model of the microscope it was hoped that more details of the lens could be obtained. This information was requested from the Nikon Corporation but with no success. Ultimately a patent application (Miwako Mandai 2006) was found through an internet search that bore very close correspondence to the specific lens. The application had been submitted by the Nikon Corporation shortly before the lens became available on the market and the 
specifications corresponded exactly with the publicized lens specifications. Assuming that no further modifications had been made to the design prior to production, it was taken that the application was a match to the lens.

As well as lens data and diagrams of internal structure, the application gave background information, explanation of the design, and graphs illustrating the lens performance. The radius of curvature, thickness and refractive index of each surface was specified and these were used to model the lens with an adjacent immersion oil layer and coverslip in Zemax software. The Nikon objective lens included a rotating collar to correct for the spherical aberrations that can be caused by the change of refractive index of immersion oil resulting from temperature changes. In the zemax model, the lens spacing was set to correct for a temperature of $23{ }^{\circ} \mathrm{C}$, the temperature for which the immersion oil refractive index was specified. Lens diagrams from the patent application and Zemax model are presented below (Figure 70 and Figure 71 respectively). In the computer model, collimated rays are traced through the back aperture to indicate the path of light through the lens. 


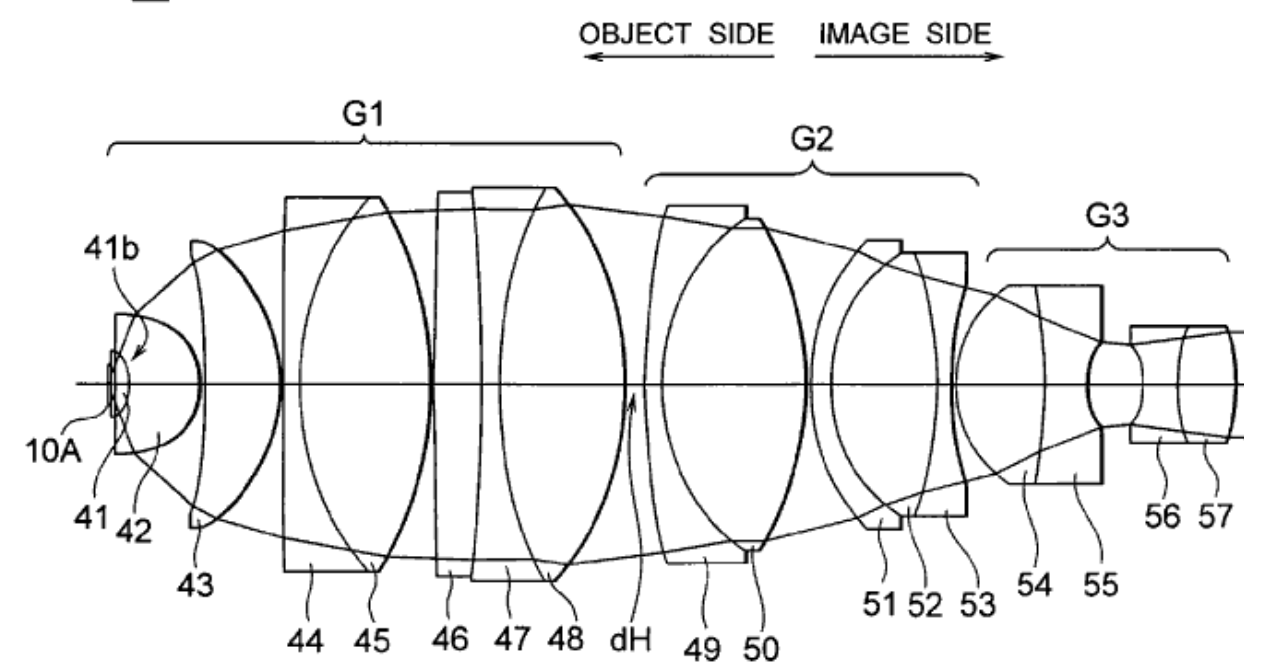

Figure 70 Diagram of lenses within the Objective lens from the patent application (Miwako Mandai 2006).

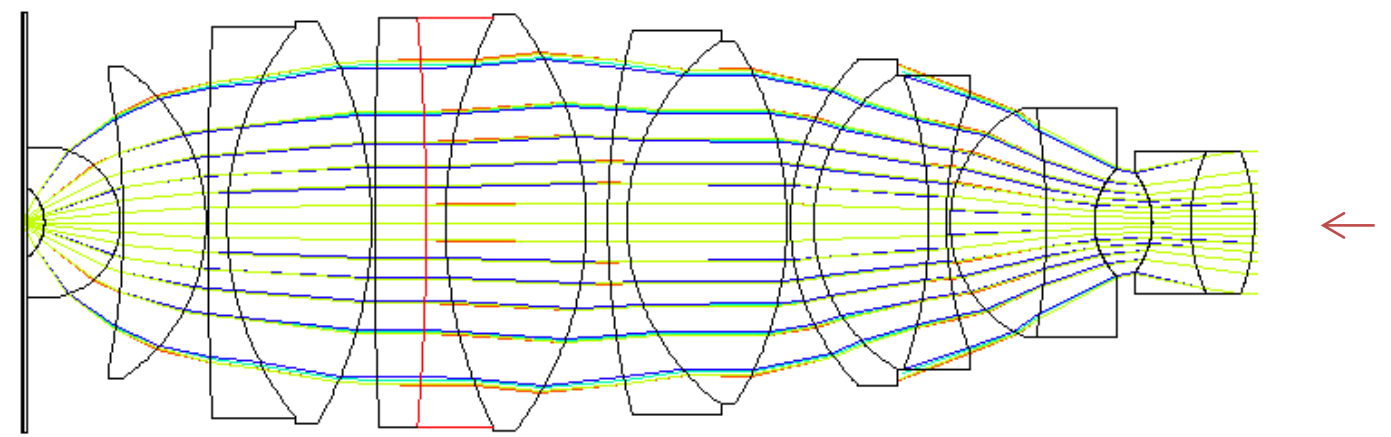

Figure 71 Diagram of the objective lens as modelled in Zemax software with information from patent application.

Since the diameters of lens surfaces were not specified it was necessary to determine the aperture diameters through physical measurement. An upper limit to all lens diameters was found from the barrel diameter of $30 \mathrm{~mm}$ and the back aperture was measured as $7.5 \mathrm{~mm}$. All remaining surface diameters were made to look similar to those in the lens diagram of the patent application. The back aperture was inspected from about one meter away and the exit pupil appeared to be about half the back aperture 
diameter. This indicated that the back aperture was not the limiting aperture of the system.

A combined model of the objective lens, immersion oil and coverslip was desired. The thickness and refractive index of the coverslip were found in the product literature. The refractive index of the immersion oil was also readily available but the thickness of the oil layer during imaging was not. Ray tracing was carried out with a range of oil thicknesses to determine which resulted in a beam focussed on the coverslip-sample interface (Figure 72). The wavelengths associated with the rays were set as $460 \mathrm{~nm}$, $500 \mathrm{~nm}, 565 \mathrm{~nm}$ and $600 \mathrm{~nm}$, to correspond to the edges of bands in filters being used in the physical microscope at the time.

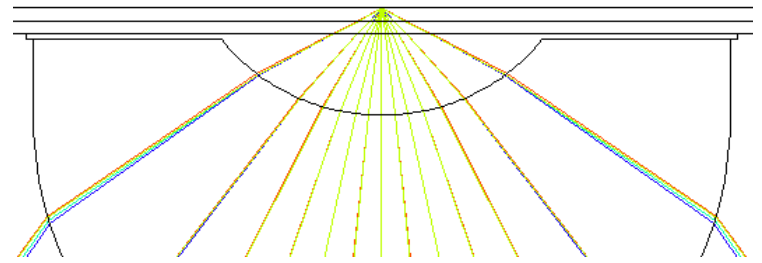

Figure 72 Diagram exhibiting rays being focussed upon the interface of the coverslip and media as modelled in Zemax.

Numerous generic lens analyses could be applied to the lens model. One analysis found the lens model to have an effective focal length (EFL) of $2 \mathrm{~mm}$, in agreement with the Nikon objective. The results output figures of some further analyses characterising aberrations are presented in Figure 73 and Figure 74. 


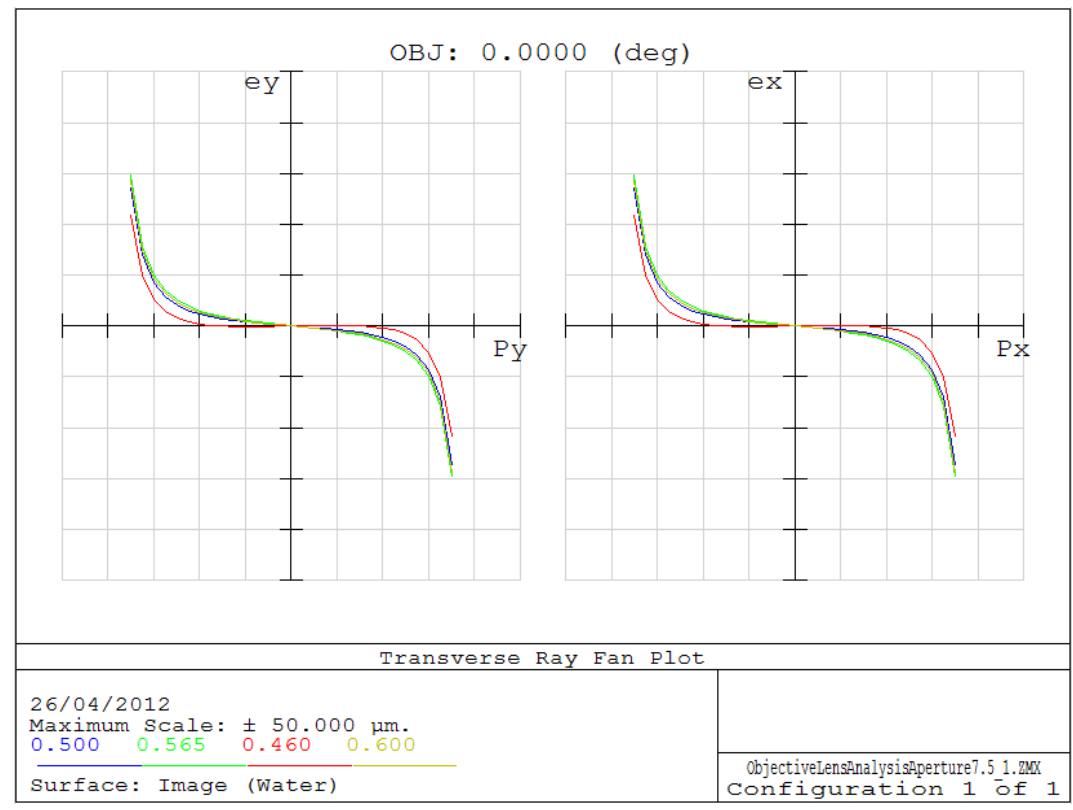

Figure 73 Transverse Ray Fan Plot generated within Zemax. This shows the lateral displacement of rays from the focus as a function of their displacement from the optical axis upon entry to the lens. This suggests that in TIRF mode, the lateral position of the Evanescant field may shift as the entry beam moves off axis and the incident angle at the coverslip interface nears the maximal value.

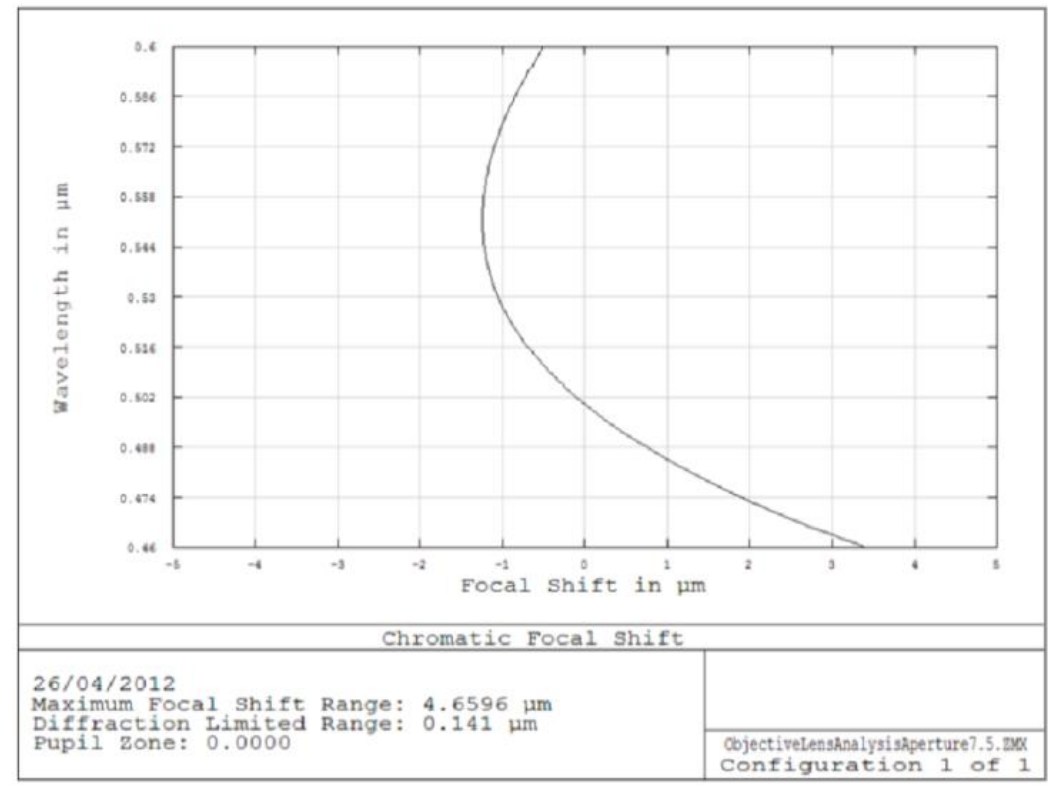

Figure 74 This Chromatic Focal Shift graph presents the shift in focal length with wavelength. It suggests that beams may need to be focussed independently to co-focus. 
Through ray tracing, measurements were made to locate the positions of the front and back focal planes. The front focal plane relative to front lens surface and the back focal plane relative to the back aperture are presented in Figure 75 and Figure 76 respectively.

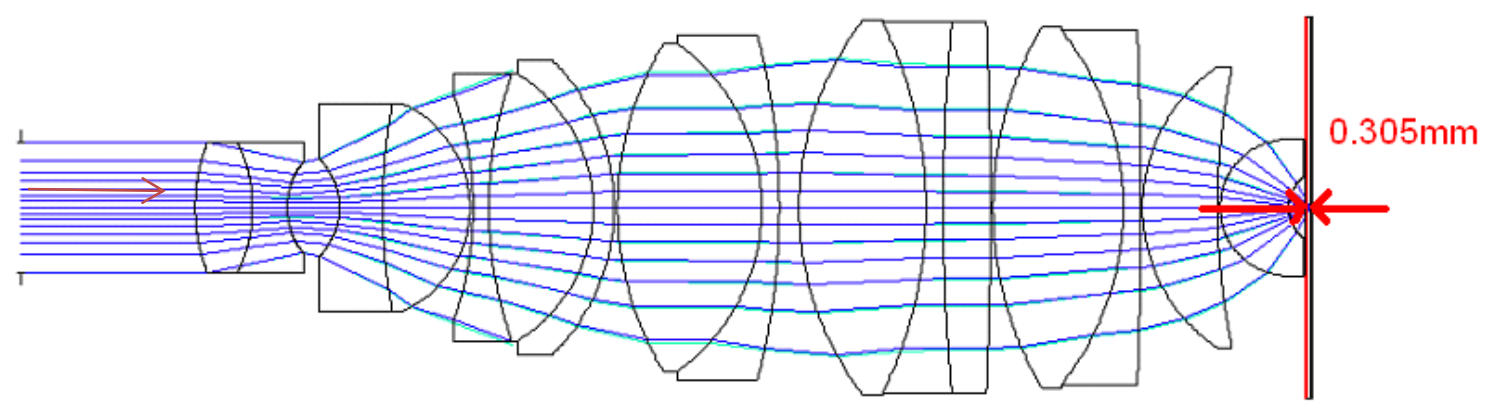

Figure 75 Ray tracing applied to determine the distance from the front surface of the objective lens to the coverslip-water interface.

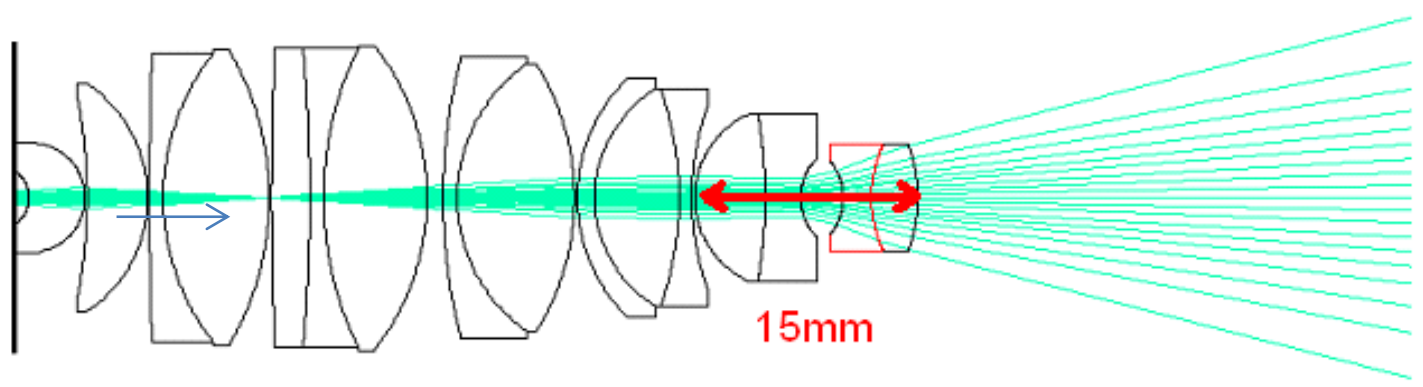

Figure 76 Application of ray tracing to determine the position of the back focal plane relative to the back aperture of the objective lens. 


\subsection{Beam Steering}

In practice, alignment is a process of trial and improvement. If it could be done prescriptively it would save time and enhance reproducibility. With the support of raytracing software it may be possible. The key technique is to trace rays in the reverse direction, essentially defining a desired outcome in order to determine the necessary cause.

Reverse ray-tracing was carried out to explore the necessary lens positioning to achieve a desired sample illumination. Control lenses were added on a common axis to the model of the Nikon objective lens and their spacing was optimised using a programed merit function. The desired illumination was then traced backwards through the lens system (Figure 77).

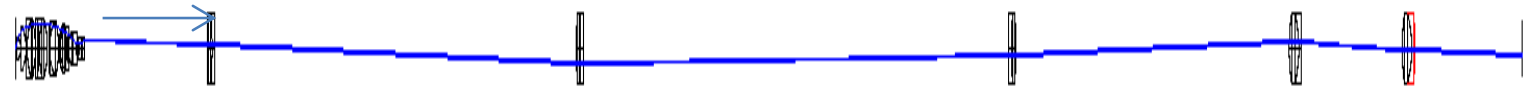

Figure 77 Reverse ray-tracing from desired sample illumination through lenses all on a comon axis.

Two beam control lenses were then moved the lateral distances necessary to produce the trajectory emanating from the source in the actual instrument (Figure 78). Values of displacement could be read from the software.

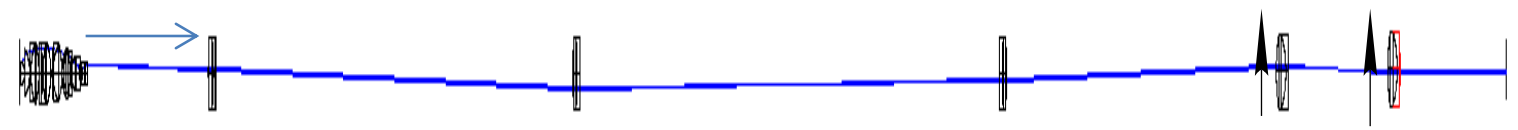

Figure 78 Two steering lenses are moved by a known distance laterally to make the beam ultimately follow the axis.

The ability to do this relies on being able to specify positions of components in reality as in the model. Through ray tracing analysis of an optical design one could 
determine the necessary precision of positioning prior to building the instrument and verify its feasibility.

\subsubsection{Evanescent Field Specification for Highest Membrane-Cytoplasm Excitation Ratio}

There are subtleties to the understanding of the evanescent field. Counterintuitively, the evanescent intensity beyond the interface can be greater than the incident intensity of the beam striking the interface (Axelrod, Burghardt et al. 1984). Also, the incident beam intensity will drop as incident angle increases (see Figure 27), due to increasing eccentricity of the intersection of beam and interface. A simplified model was made to investigate how the evanescent field could be generated to maximise membrane-cytoplasm excitation ratio (Figure 79).

\section{Evanescent Field Penetration}

Evanescent Field Intensity

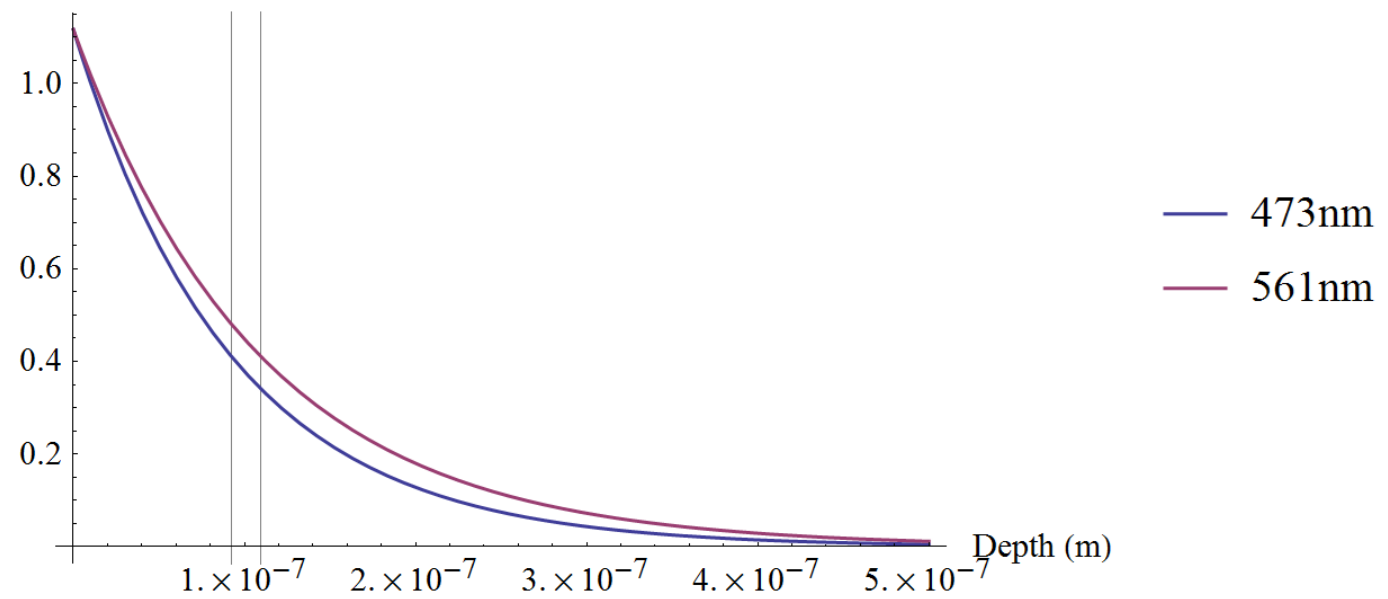

Figure 79 The field intensity against distance from the interface for two wavelengths at the same incident angle. The verticle gray lines indicate the penetration depth for each wavelength. 
An objective function was written to maximise the absolute value of membrane excitation and the ratio of membrane to cytoplasm excitation ( membraneExcitation ${ }^{2} /$ cytoplasmExcitation ). Excitation was approximated by integrals of the evanescent field intensity up to and beyond the thickness of the cell envelope. An optimum angle of incidence (around 67 degrees) was found that was neither sensitive to changing the incident wavelength from $473 \mathrm{~nm}$ to $561 \mathrm{~nm}$ (Figure 80 ), nor from changing the cell envelope thickness from 30nm (Hobot, Carlemalm et al. 1984) to 100nm (Figure 81).

Optimum Incident Angles For Typical Wavelengths Objective Value

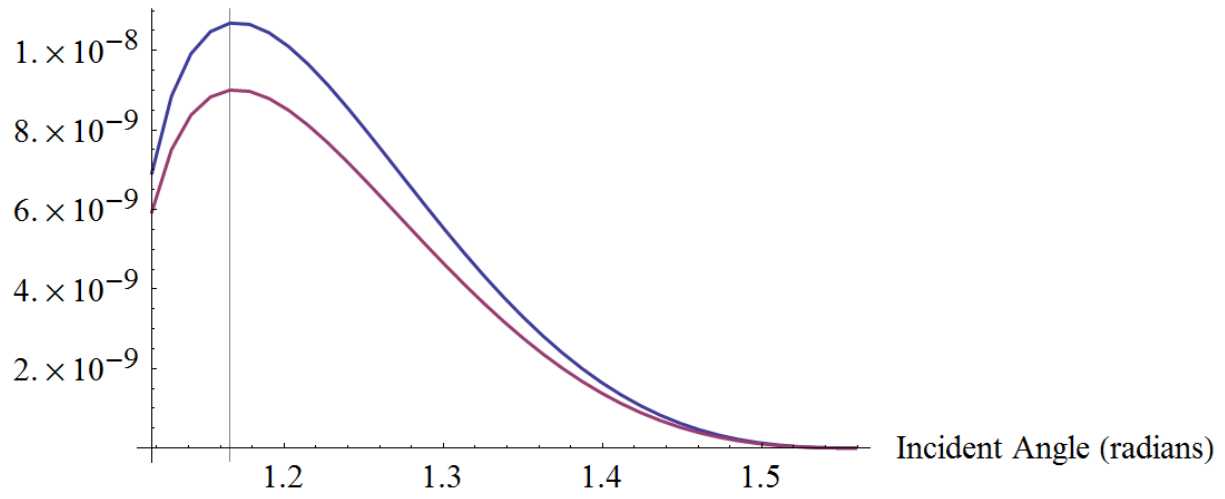

$-473 \mathrm{~nm}$

$-561 \mathrm{~nm}$

Figure 80 The change in merit function value with incident angle for two alternative illumination wavelengths. 
Optimum Incident Angles For Alternative Membrane Thicknesses

$$
\text { Objective Value }
$$

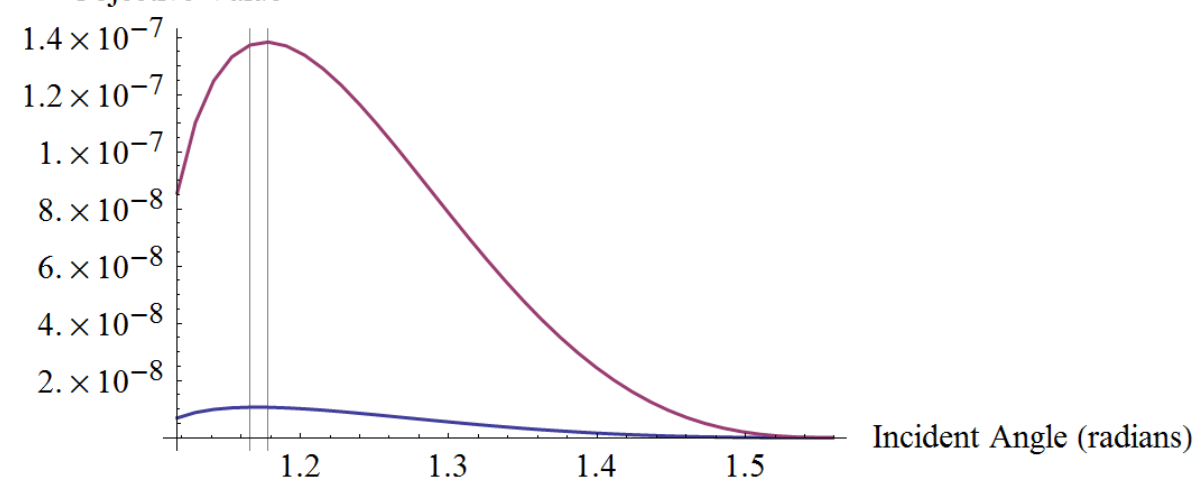

Figure 81 The change in merit function value against incident angle for two alternative cell envelope thicknesses.

A technique was developed to enable prescription of the optimal incident angle. For this a kinematic mirror mount was modified to be actuated reproducibly with a micrometer, and a device was made to measure angles incident upon the coverslip. A series of respective measurements were taken and the results automatically plotted for convenience (Figure 82). The device and associated technique are described in detail in Appendix 5.1.1. 


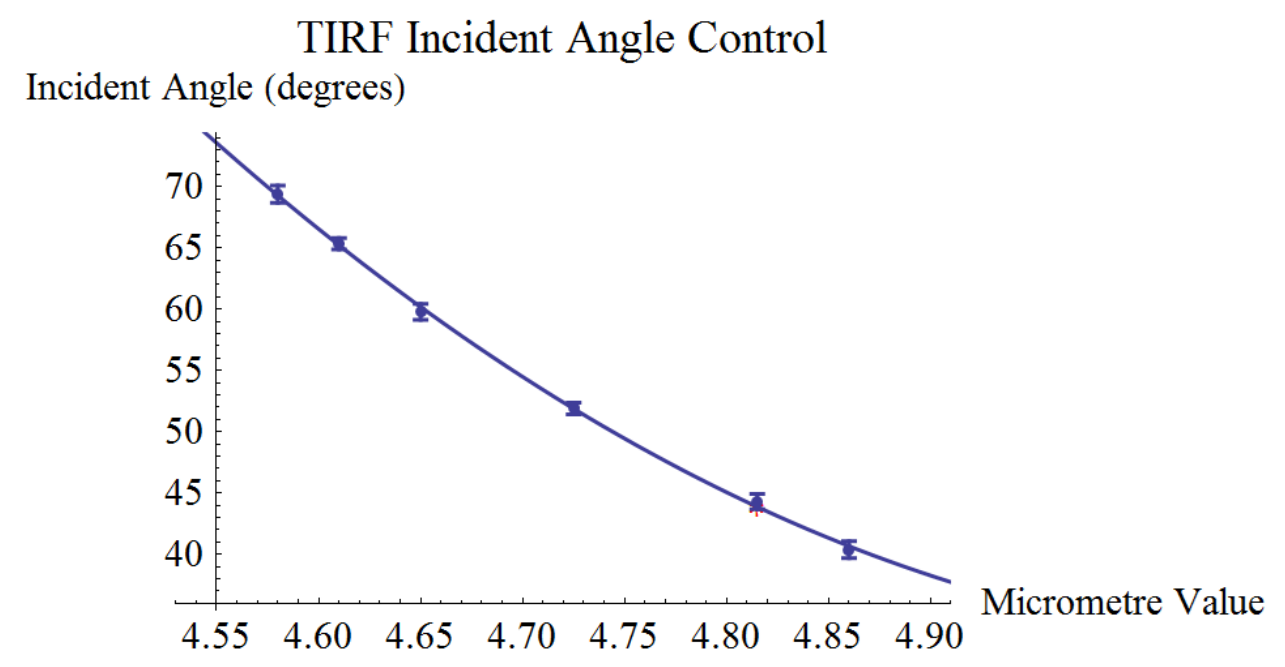

Figure 82 Example graph generated for setting the angle of incidence with a micrometer.

\subsubsection{MODEL-ASSISTED LIGHT QUANTIFICATION}

\subsubsection{Mapping of the Excitation Field in Three-Dimensions}

The intensity of fluorescence emission is used to discern a number of singlemolecule characteristics. To compare fluorescence observed in different regions in an image one must know the relative illumination intensities. This is since, up to the point of saturation, the emission is approximately proportional to the excitation. Knowing the absolute intensity would enable reproducibility of experiments. A method was therefore developed to produce a three-dimensional map of the absolute illumination intensity.

The total laser beam power entering the microscope was measured and multiplied by the measured transmission from the point of beam entry to the sample. This value was used as the total incident intensity in the model of evanescence previously described. 
The beam angle of incidence was measured and other parameters taken from the literature.

The beam was modelled to have a two-dimensional Gaussian profile at the interface and it was necessary to determine values for the $x$ and $y$ mean and standard deviations. While these parameters could have been approximated analytically, common variation in alignment dictated a need to make measurements. For this, a series of videos was taken of surface-immobilized EGFP molecules randomly distributed on a coverslip. An appropriate surface density of EGFP is evident in the left image of Figure 83 below. Ideally the molecules would be densely distributed, but each be separated by more than the Rayleigh limit.

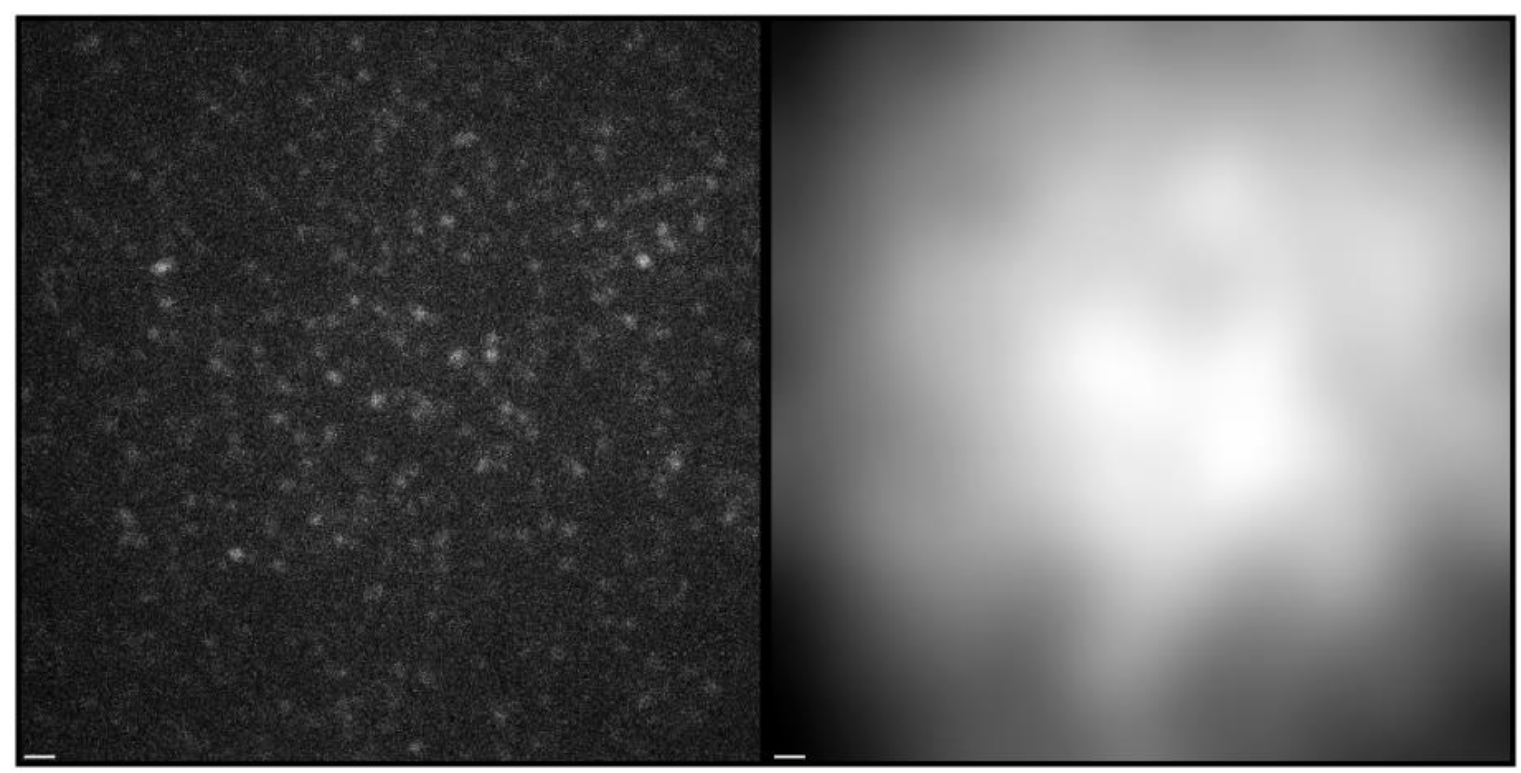

Figure 83 Images illustrative of the process of producing an illumination field intensity map. Left) TIRF illumination of randomly distributed EGFP molecules. Right) The blurred summation of five EGFP images. Scale bars $1 \mu \mathrm{m}$. 
The first illuminated frames of each video were combined and blurred to smooth local variations in intensity. A two-dimensional interpolating function was then generated (Figure 84) and evaluated at points on a grid in the $x-y$ plane.

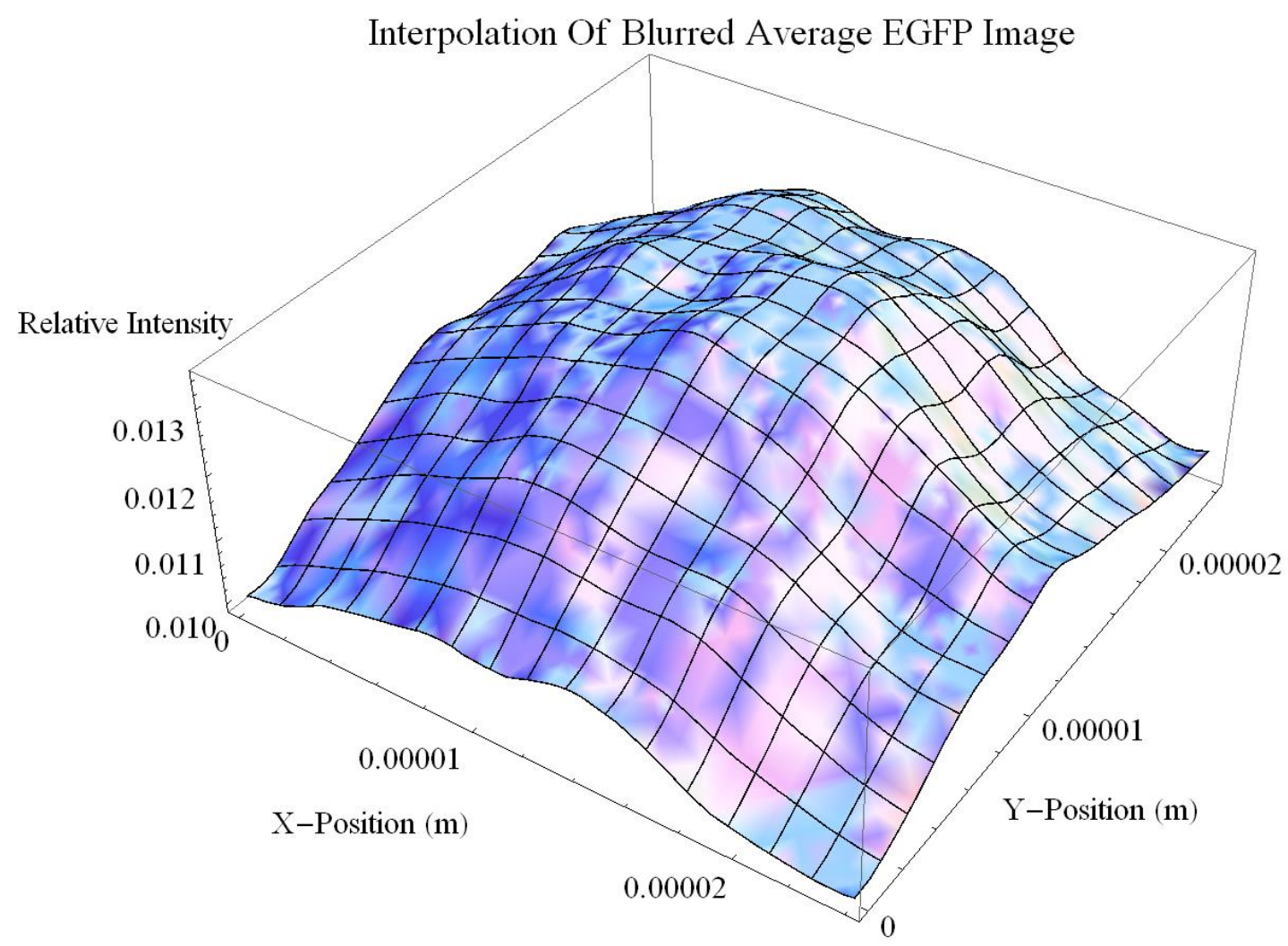

Figure 84 An interpolating function of fluorescence emission used to approximate the form of sample illumination.

A two-dimensional Gaussian function was fitted to the results (Figure 85) and fitting parameters extracted for generation of the field map. The integral under the whole surface (relating to the total evanescent intensity) and the $x$ and $y$ values of mean and standard deviation were used to parameterise the map of illumination intensity in the sample. 


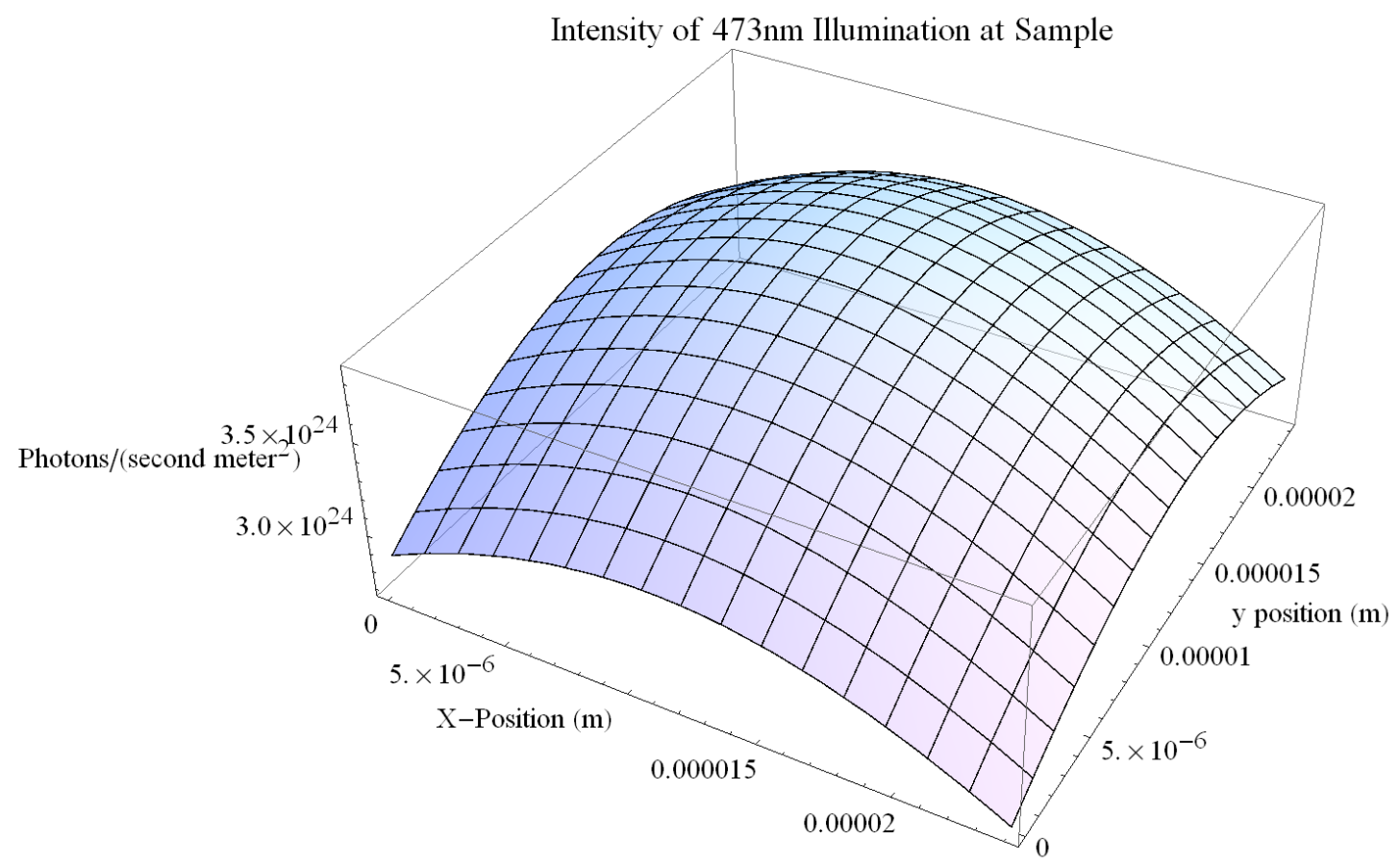

Figure 85 Absolute intensity of EGFP illumination at the focal plane.

\subsubsection{Single-Molecule Brightness}

Values for fluorophore brightness can be found in the literature and are calculated as the absorption cross-section multiplied by the quantum yield. A method was created to compute approximate brightness values through combination of single-molecule imaging (with mapped illumination intensity) and a model of the microscope emission path.

Initially sequences of images were visually inspected to identify single molecules and determine the frames for which they had been fluorescent for the entire exposure time (Figure 86). The illumination intensity of each selected fluorophore was found by 
its location in the field map. Each fluorophore was assumed to emit fluorescence isotropically and the proportion entering the objective lens was calculated from the NA. A power meter was used to measure the attenuation of a laser beam from the sample to the camera.

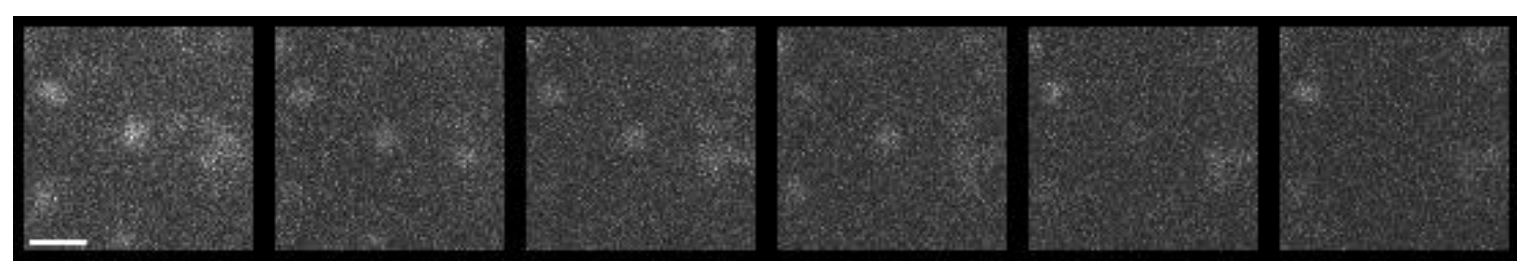

Figure 86 A sequence of micrographs (from left to right) in which a singlemolecule of EGFP (centre) is identified. Frames 3 and 4 were taken to exhibit a singlemolecule for the entirety of the exposure time and selected for analysis. Scale bar $1 \mu \mathrm{m}$.

While an estimate of emission path detection efficiency had been made from attenuation of a laser beam and the registered pixel values, it was desired that a more detailed model be made. To deduce the number of causative incident photons from the image pixel values, it was necessary to understand the workings of the EMCCD. Parameters specific to each EMCCD, the acquisition settings, and the associated saturation levels and forms of noise were considered.

While noise can be described analytically, a stochastic simulation approach was taken that generated a number of parameters from probability density functions. For example, Shot noise was inherent from frame to frame as a result of generating values randomly from a Poisson distribution. The simulated camera response was ultimately based on a combination of theory and data from videos taken with different conditions. The accuracy of the simulated background noise, including an optimised stray light 
component, was verified through comparison of histograms of real and simulated pixel values.

The PSF was modelled with a two-dimensional Gaussian distribution and given a value of standard deviation to match the images. With all other parameters set to match the experimental, the remaining parameter to calibrate with was the value of fluorophore brightness. This was modified until a simulated image was produced that was visually comparable with the measured image (Figure 87).

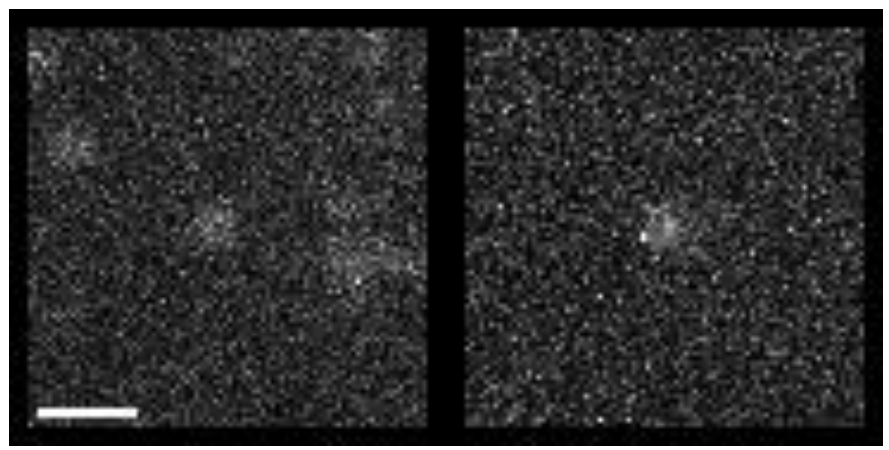

Figure 87 Left: A real image of a single EGFP molecule. Right: A simulated image calibrated to correspond to the real image. Scale bar $1 \mu \mathrm{m}$.

\subsubsection{Measurement of Photobleaching}

With an adequate SNR one often finds that photobleaching occurs too rapidly to capture enough video frames of molecular activity. For example one may wish to track a molecule to characterise its mobility, but photobleaching renders it invisible before enough trajectory information can be gained. A validated model of photobleaching could be used to explore how conditions may be altered to extend the number of frames for which SNR is adequate. In the case of two-colour imaging one may determine how to 
achieve maximum SNR for each colour and have both bleach after the same period of time.

A method was devised to measure the dependence of single-molecule photobleaching on illumination intensity using the same videos as for field mapping. Maps were produced for all frames in the video sequences and interpolated as before (Figure 88).

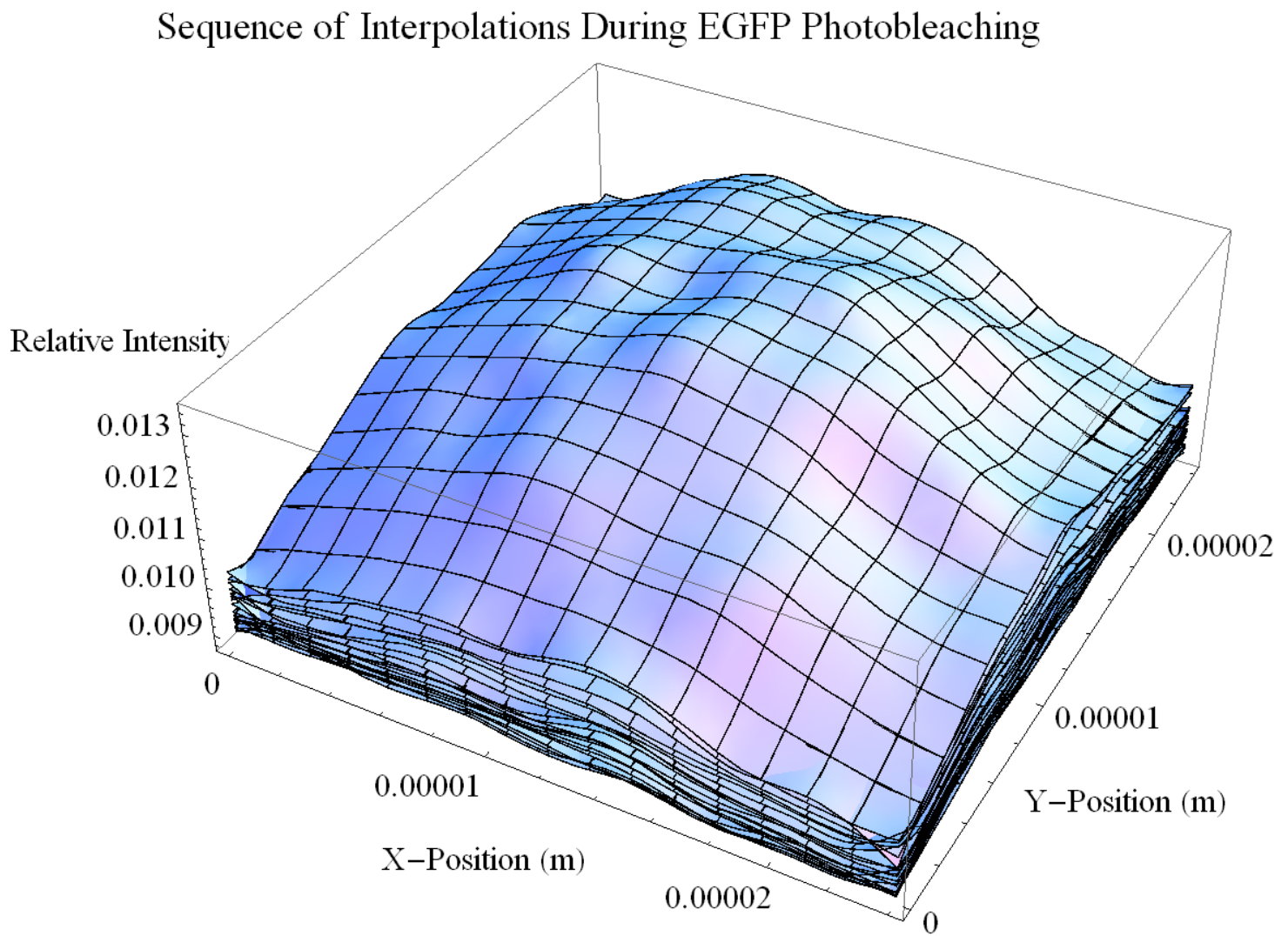

Figure 88 Frame-by-frame photobleaching illustrated by reduction in interpolated fluorescence emission. 
The interpolating functions were then evaluated for each frame at a grid of points in the $x-y$ plane. The values were plotted, revealing bleaching that was well described by exponential decay (Figure 89).

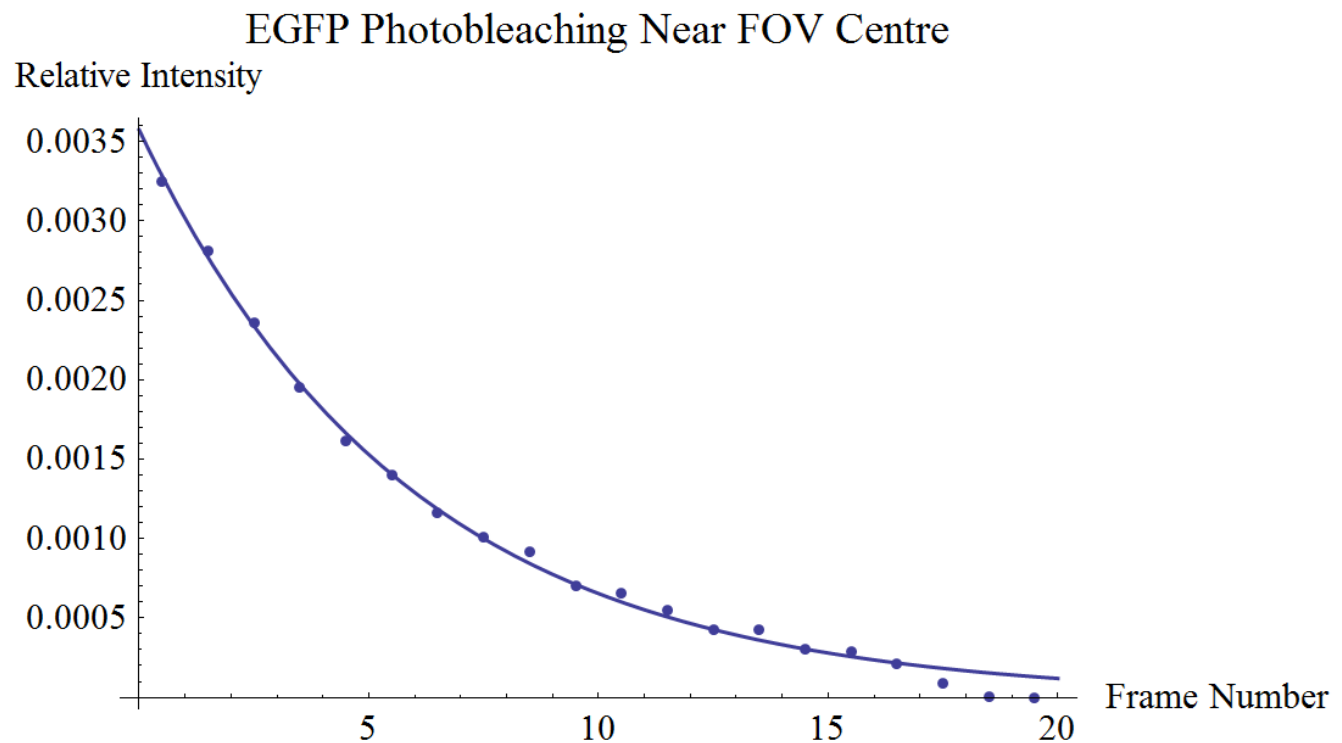

Figure 89 Photobleaching exhibited in an intensity time trace from near the centre of the image. Exposure time. 30ms.

For each curve an exponential function was automatically fitted and the decay rate produced. These values were then plotted against their respective field intensities and a linear model was fitted. The gradient was taken as the decay constant specific to EGFP in a basic exponential decay model to describe photobleaching (Figure 90). 
Linear Fit Of EGFP Photobleaching Rate Against Illumination Intensity Decay Constant $*$ Photons $/\left(\mathrm{s} * m^{2}\right)$

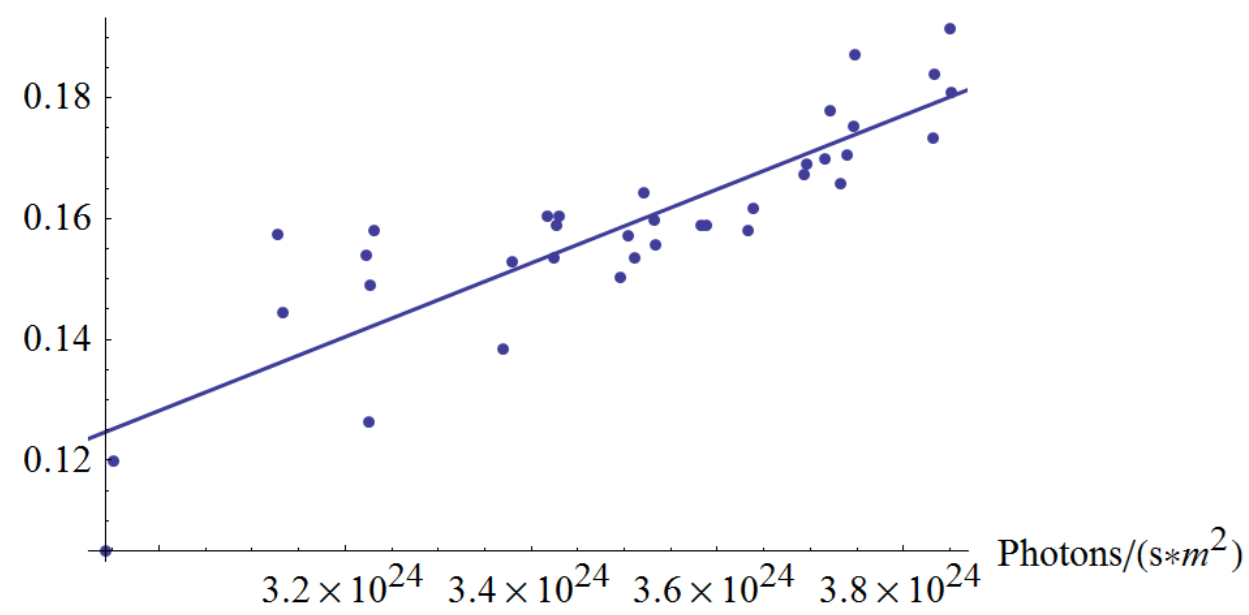

Figure 90 The photobleaching rate and respective illumination intensity for each exponential fit to the data presented in Figure 88. Here photobleaching rate =decay constant times illumination intensity. In this case the decay constant was calculated as $6.1 \times 10^{-26}$.

\subsubsection{EXPERIMENT SIMULATION FOR IMPROVED IMAGING DATA}

\subsubsection{Modelling the Whole Experimental System}

A key idea in Systems Engineering is that of "sub-optimisation". This is where the optimised behaviour of a subsystem may lead to a sub-optimal performance at the system level. So far, only subsystem models have been presented. A grand aim of the research was the generation and use of a unified model describing significant aspects of the experiment from the sample illumination to the single-molecule response and detection at the cameras. 
The aim was to make the most of the broad range of quantitative data relevant to experiments, much of which is not currently considered in a systematic manner. Perhaps, by making use of computer solving power as opposed to (or as well as) the usual approach of reasoned experimental trial and improvement, better system performance can be obtained. With a validated whole experiment simulation model, one would not need to speculate on the effect of changing a parameter. Rapid processing and efficient search algorithms could be applied to find optimal parameter settings for a given experimental goal. Sensitivity analysis, varying each parameter independently to determine the magnitude of the effect on image quality, could be used to rank parameter significance. This in turn could be used to prioritise acquisition of improved components or to better inform the process of architecting.

If members of a research microscopy group developed a common fluency in computational modelling, one could imagine work being centred on maintenance and improvement of a model representing the shared understanding of the experimental system. This model would serve as the central artefact of hypothesis, instrument control, and analysis. Rigorous falsification through real world experimentation would be fundamental since computational models can be as easily misinterpreted and as flawed as mental models. A model can be inadequate through omission of important system features or due to bugs in the code and the scope for error increases with the number of model inputs. Given a model that correctly describes a system, simulations results could be misleading or inaccurate due to chaotic behaviour or numerical errors respectively. To build a system model with both generality and accuracy, Physics-based symbolic models 
would be parameterised wherever possible with experimental results. In cases where appropriate symbolic descriptions were not found, empirical and statistical models would be used.

Careful modelling will force one to be explicit about the assumptions and extent of knowledge of an experiment. If the modelling is "clean" (Martin 2008), the experimental reasoning will then be transparent and could be improved by anyone with expertise and a digital copy. The approach would enforce clarity of thought and enable concepts to be advanced when new information comes to light. The process of computational modelling may reveal simplifying structures that clarify understanding of the system.

Though (physics-based and empirical) models of component mechanisms abound for phenomena in the experimental system, there are few examples of attempts to unify such models for the purpose of improved whole system configuration ((Mathies, Peck et al. 1990) is of relevance). The validity of modelling the combined live-cell and experimental system has not been established. The complexity of the living biological sample may prove to be a limiting factor as the chemical environment is known to effect fluorophore activity (Lichtman and Conchello 2005). An open source software platform for the modelling of living cells has been developed (Loew and Schaff 2001). Features enable dynamic simulation based upon specified internal geometries, species concentrations and reaction rates.

Two general and potentially applicable approaches used in industry are "Multidisciplinary Design Optimisation" (MDO) (Agte, de Weck et al. 2010) and "Design of Experiments" (DoE (Antony 2003)). In the nomenclature of MDO, the 
purpose of the experimental system model is "Design Optimisation". This is the quantitative analysis of the effect of "design variable" changes in a given model architecture with the aim of improving system performance. The MDO mathematical formalism (based on Nonlinear Programming) for this process is presented below in Figure 91. If the system is described using this formalism, numerous optimisation techniques become conveniently applicable.

$$
\begin{aligned}
& \mathbf{J}=\left[J_{1}(\mathbf{x}, \mathbf{p}) \ldots J_{z}(\mathbf{x}, \mathbf{p})\right]^{T} \\
& \mathbf{g}=\left[g_{1}(\mathbf{x}, \mathbf{p}) \ldots g_{m 1}(\mathbf{x}, \mathbf{p})\right]^{T} \\
& \mathbf{h}=\left[h_{1}(\mathbf{x}, \mathbf{p}) \ldots h_{m 2}(\mathbf{x}, \mathbf{p})\right]^{T} \\
& \mathbf{x}=\left[x_{1} \ldots x_{i} \ldots x_{n 1}\right]^{T} \\
& \mathbf{p}=\left[p_{1} \ldots p_{i} \ldots p_{n 2}\right]^{T} \\
& \min \mathbf{J}(\mathbf{x}, \mathbf{p}) \\
& \mathbf{g}(\mathbf{x}, \mathbf{p}) \leq 0 \\
& \mathbf{h}(\mathbf{x}, \mathbf{p})=0 \\
& x_{i, L B} \leq x_{i} \leq x_{i, U B},(i=1, \ldots, n 1)
\end{aligned}
$$

Figure 91 The MDO mathematical formulation for system modelling and optimisation. Here, $\boldsymbol{J}$ holds the system level objective functions (e.g. SNR), $\boldsymbol{g}$ and $\boldsymbol{h}$ hold system inequality and equality constraints respectively, $\boldsymbol{x}$ holds the configuration "design variables" (the changeable parameters), and $\boldsymbol{p}$ holds the static parameters. $x_{i, L B}$ and $x_{i, U B}$ represent the lower and upper bounds of design variable values for each degree of freedom.

Examples follow of how, in the context of MDO, a fluorescence microscopy experiment could be described:

An objective function, $J_{l}$, to be minimized could be the reciprocal of the SNR.

An inequality constraint, $g_{l}(\boldsymbol{x}, \boldsymbol{p})$, could be that the negative of the lateral spacing between two single molecules, plus the Rayleigh limit distance, must be equal to or less than zero. 
An equality constraint, $h_{1}(\boldsymbol{x}, \boldsymbol{p})$, could be that the difference in penetration depth of each evanescent field must equal zero.

A design variable, $x_{1}$, could be the output power of one laser source.

Associated bounds, $x_{1, L B}$ and $x_{1, U B}$, could be the minimal and maximal stable output power of the laser source.

A parameter, $p_{1}$, could be the NA of the Objective lens.

\subsubsection{The Model Implementation}

From initial experiences modelling the optical system with Zemax software it became apparent that a large amount of effort could potentially go into maintenance of a simulation model. A decision was made to make use of a high level, interpreted programming language to minimally model features of the experimental system. An experimental system model was first written in the Matlab computing environment using its object-oriented capability. It was later desired that the model should have a much more symbolic flavour and a second model was written in the Mathematica computing environment. The ease of manipulating parameters in analytical expression would make exploration of the parameter space much more intuitive for members of a research group. Some effort was made to carefully name each variable so the code could be readable by a group member without significant programming experience.

The code is divided for intelligibility, with sections containing the purpose built functions, preliminary parameters, the controller, and the simulation model. The use of generalised functions reduces repetition in the code and clarifies what is specific to each 
circumstance of a function's use. It also enables minimal rewriting when aspects of the model (i.e. the experimental understanding) are improved. The format of Mathematica is well suited to the application because the programme can be broken into individual expressions that can be evaluated individually or as groups. This enables simple extraction and plotting of data internal to the programme. Furthermore, the components can each be tested for correctness or for the time taken to evaluate which is useful when debugging and improving program efficiency. Using the Mathematica "Notebook" format enables diagrams and references to be embedded in the code to further clarify the experimental system model and enable the tracking of information sources. The parameters that can be controlled during an experiment are presented in a graphical user interface in an order related to the passage of light through the microscope (Figure 92). 

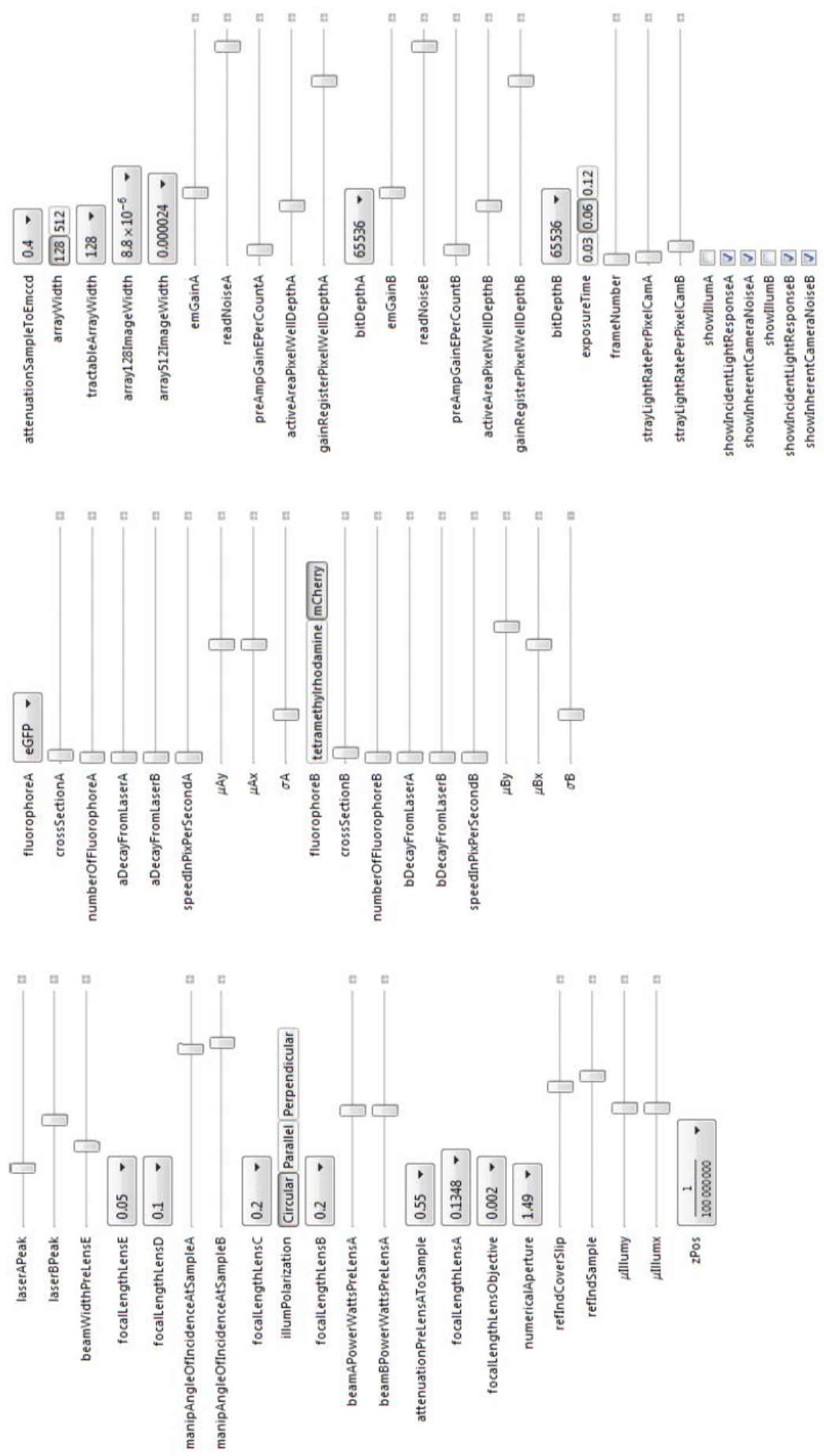

Figure 92 Image showing all parameter controls for the whole experiment simulation. 
System boundaries are artificial and judgement must be exercised in the extent of what is modelled. Currently around sixty parameters are considered, many of which are general to fluorescence microscopy and some of which are specific to the instrument and samples. Features have been included that are pertinent to my experimentation such as the ability to trace bleaching of numerous fluorophores in a point spread function and the blurring associated with molecular mobility.

Some aspects of the experiment including all forms of noise are stochastic in nature. This stochasticity was modelled by pseudo-random generation of values from probability distributions. By simulating multiple times, this errors associated with each stochastic output value could be calculated.

The peak wavelengths of the illumination beams are specified and the resulting spectra are plotted along with the absorption and emission spectra of the selected fluorophores (Figure 93). Inspection of this graph enables qualitative identification of suitable illumination wavelengths. Quantitative evaluation is enabled with the plots of detected light attributed to signal (Figure 94), bleedthrough (Figure 95), and crosstalk (Figure 96). These plots indicate the ultimate recorded signal spectra calculated in the simulation from the interaction of the specified illumination with illumination path filters and fluorescent protein absorption spectra, and the transmission and detection of fluorescence in the emission path. 


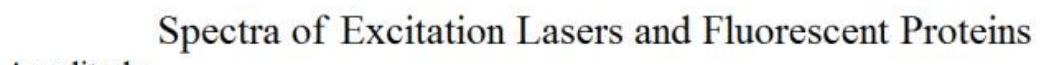

Amplitude

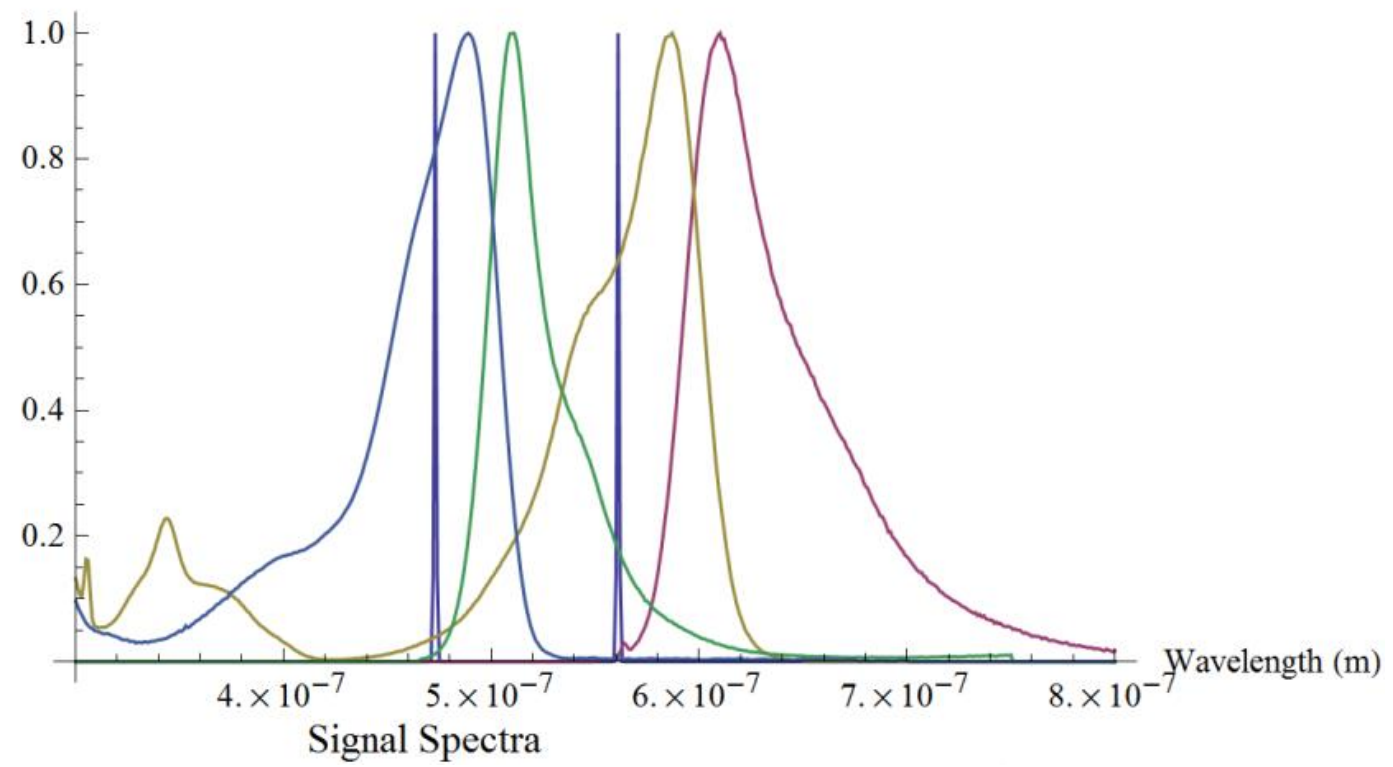

Figure 93 Graph plotted in graphical user interface for comparison of the spectra of illumination beams and fluorescence.

\section{Relative Intensity}

\section{Signal Spectra}

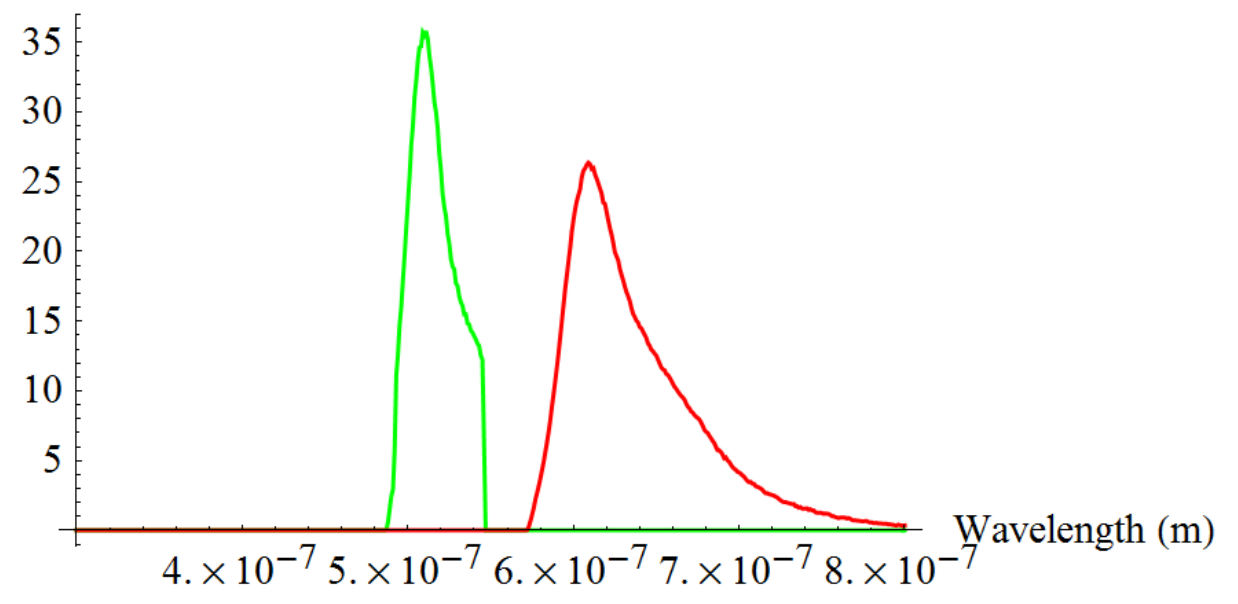

Figure 94 The calculated spectra of desired fluorescence emission, detected by the images sensor given the system parameters. 
Relative Intensity

\section{Bleedthrough Spectra}

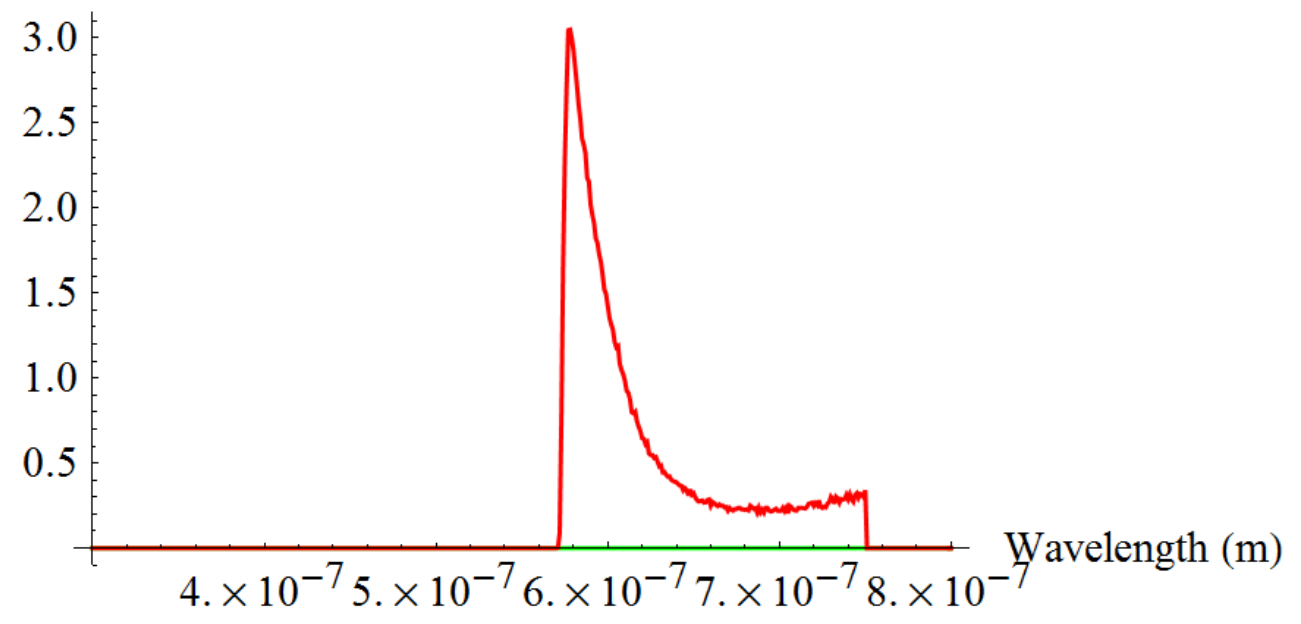

Figure 95 The calculated spectra of bleedthrough fluorescence emission, detected by the images sensor given the system parameters.

Relative Intensity

\section{Crosstalk}

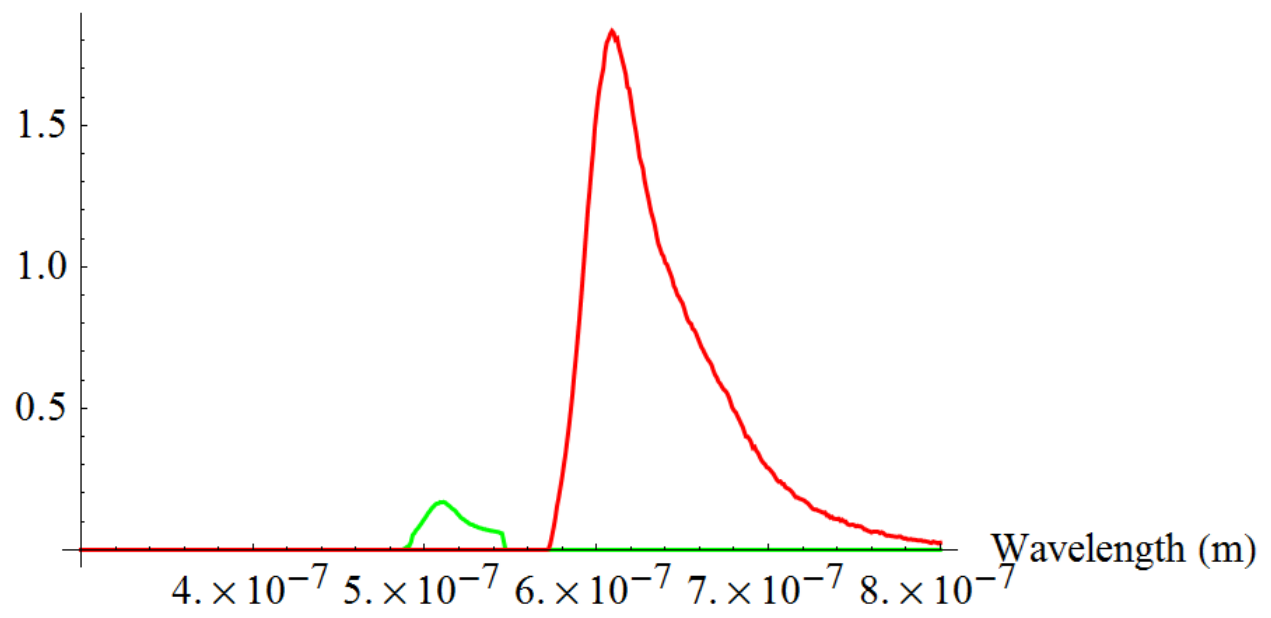

Figure 96 The computed spectra of fluorescence emission resulting from crosstalk, detected by the images sensor given the system parameters. 
The Gaussian beam width is specified and the ultimate profile at the sample (of collimated light) is determined through geometric optics calculations given the choice of illumination path lenses. The resulting beam profile can be monitored in the simulated images and through selecting different lenses the profile can be tailored with respect to the spatial dimensions of the specimen. Further controllable parameters that affect the evanescent field include the angle of incidence (see Figure 98), the polarisation state and the refractive indices of the coverslip and sample media.

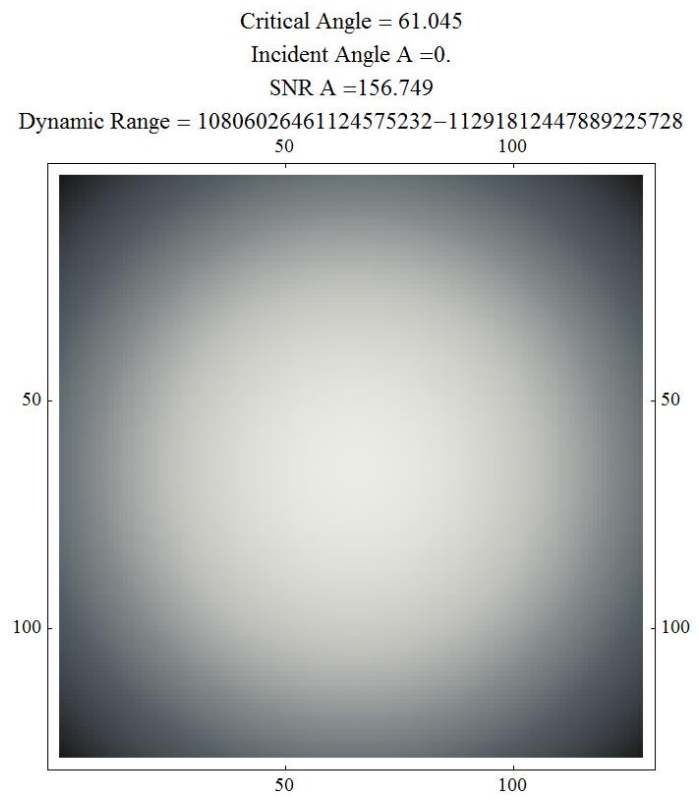

Figure 97 The simulation enables evaluation of the epifluorescence illumination beam profile at the image plane for specified system parameters. Values on the $\mathrm{x}$ - and $\mathrm{y}$ axes correspond to pixel columns and rows respectively. 


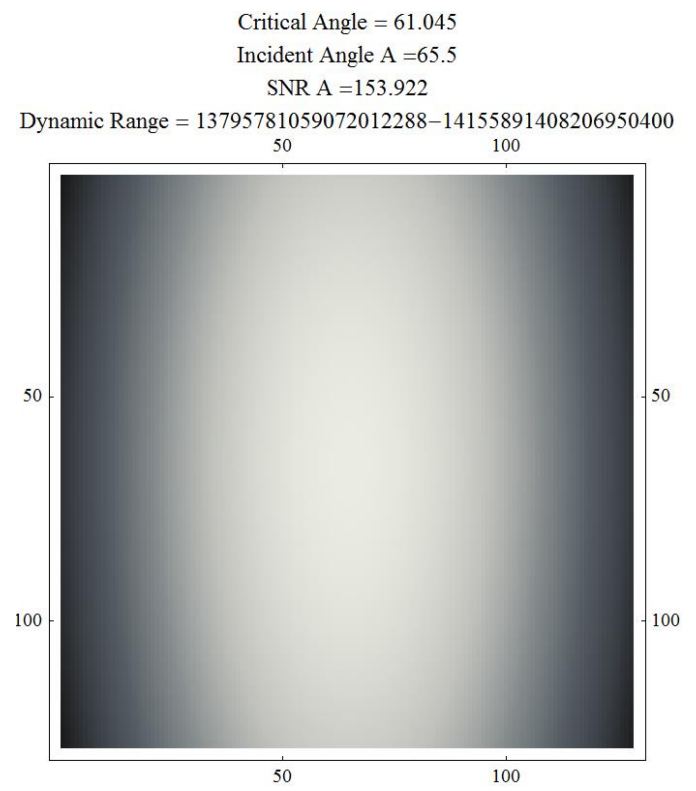

Figure 98 The effect of changing parameters can be evaluated. In this case the angle of incidence was modified to result in an eccentric TIRF illumination profile. Values on the $\mathrm{x}$ - and $\mathrm{y}$-axes correspond to pixel columns and rows respectively.

The resulting evanescent field strengths are observable through the fluorescence responses of fluorophores that can be positioned centrally (Figure 99) and laterally displaced in the field of view (Figure 100) and displaced from the coverslip-media interface (Figure 101).

Fluorophore images are approximated by two-dimensional Gaussian distributions. The standard deviations that can be set to that of the microscope PSF centroid and ellipticity set to correspond to the blurring of mobile molecules observed in the specified exposure time (Figure 102). 


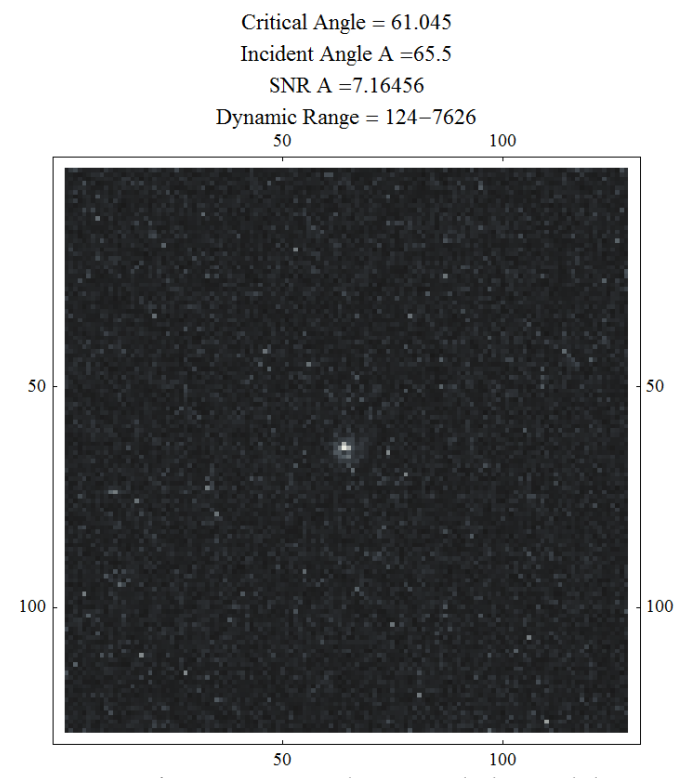

Figure 99 The model enables evaluation of the effect on the illumination field on the detected fluorescence emission of a fluorophore. The background noise is also simulated. Values on the $\mathrm{x}$ - and $\mathrm{y}$-axes correspond to pixel columns and rows respectively.

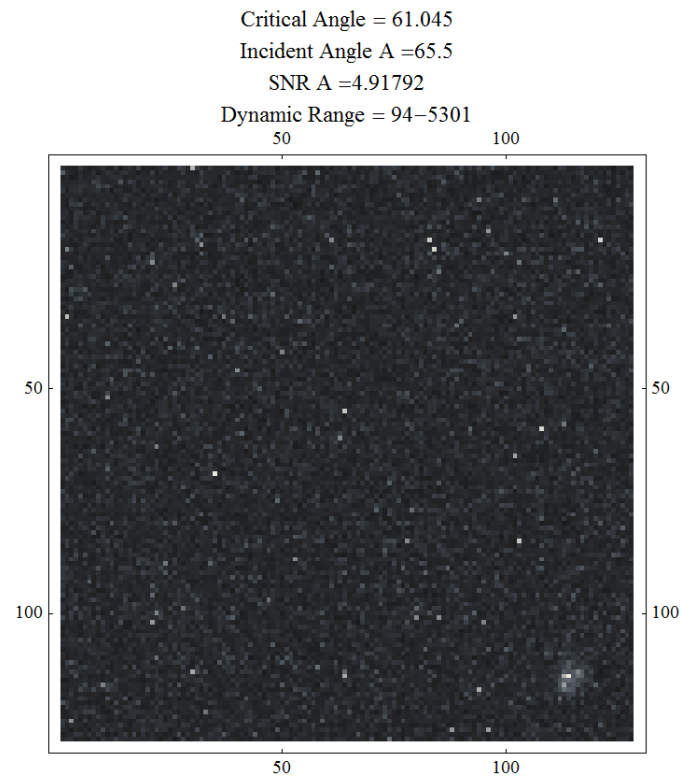

Figure 100 The fluorophore can be repositioned in the focal plane for evaluation on the effect of SNR. In this case the pixel displaced from the centre by 50 pixels along each axis. Values on the $\mathrm{x}$ - and $\mathrm{y}$-axes correspond to pixel columns and rows respectively. 


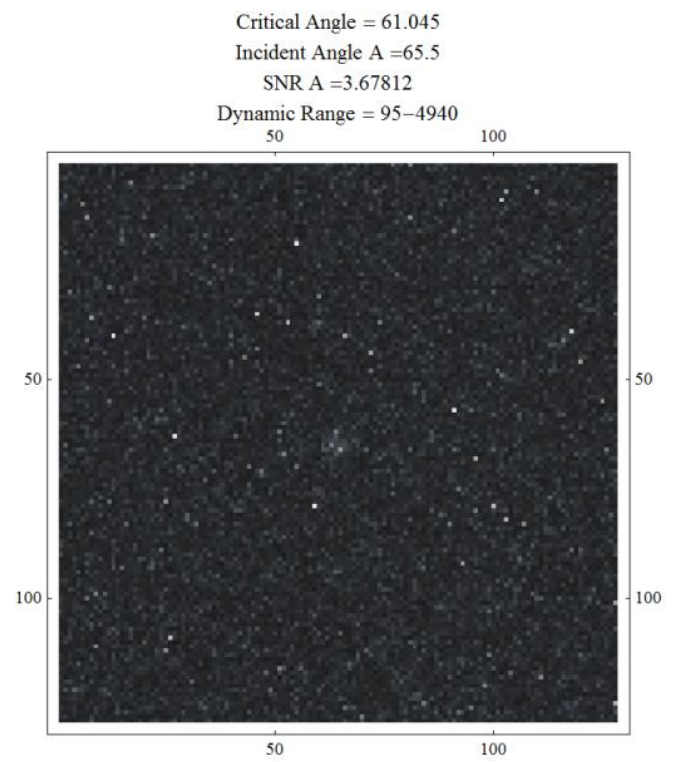

Figure 101 The fluorophore can be vertically displaced from the coverslip-water interface. This can be used to evaluate change in SNR resulting from the change in evanescent field strength. Here the fluorophore is displaced 100nm from the interface unlike all other cases where the displacement was $10 \mathrm{~nm}$. Values on the $\mathrm{x}$ - and $\mathrm{y}$-axes correspond to pixel columns and rows respectively.

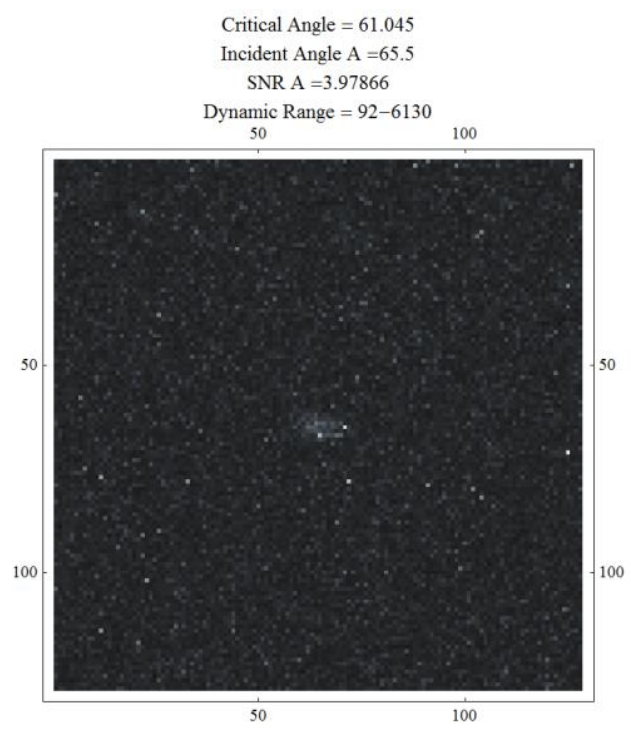

Figure 102 The blurring effect of fluorophore translation can be evaluated by specifying lateral fluorophore velocity. In this example one fluorophore moves 50 pixels/second, exposure time 0.06 seconds. Values on the $\mathrm{x}$ - and $\mathrm{y}$-axes correspond to pixel columns and rows respectively. 
The background image noise can be simulated from an analytical noise model taking into account the various gain levels, or more simply from the histogram of a real image (Figure 103). The emission path beam attenuation is specified along with the characteristics and settings of the imaging sensors. The effect of these settings on the saturation of each EMCCD is observable in a bar chart (Figure 104). The key system response of SNR is observable qualitatively in the simulated images with an associated numerical approximation.

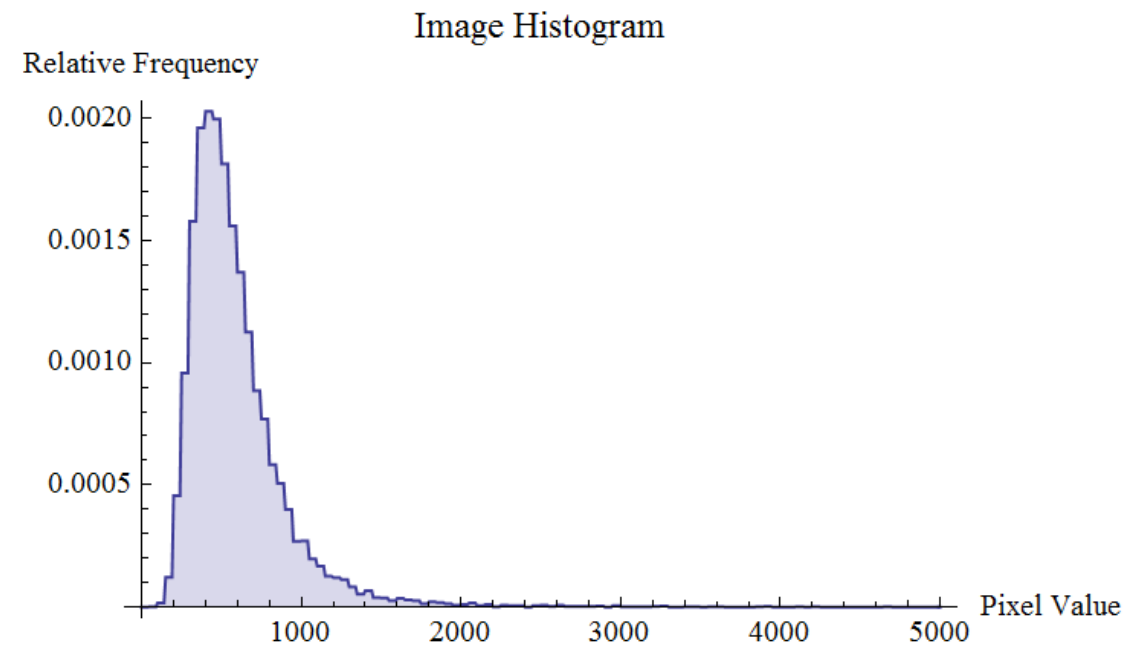

Figure 103 As part of the program output, a histogram was produced of the simulated-image pixel values. This histogram was of use in comparison with the histogram generated from pixel values in a corresponding real experimental-image. Comparison was used to verify that the model's description of noise from the specified conditions was accurate. 


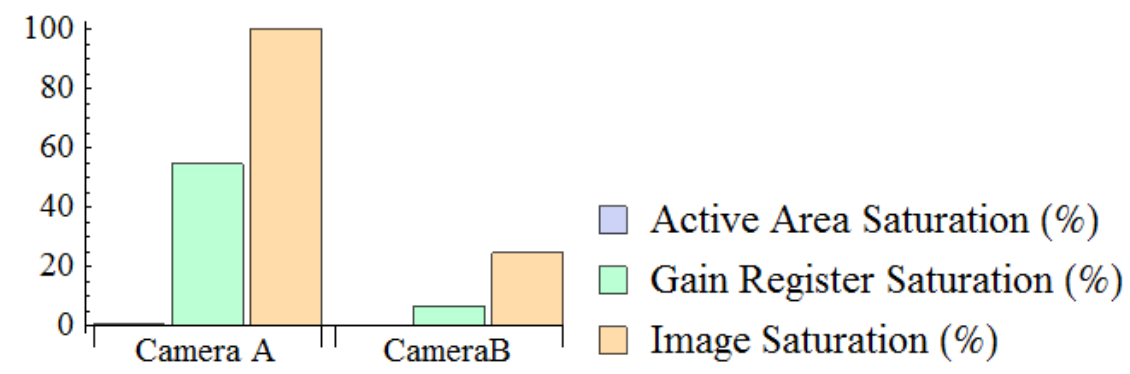

Figure 104 In order not to lose any information, it is necessary to ensure that no saturation occurs in the EMCCD. Saturation can occur at three stages in the electronics; first in the pixels, then in the gain register, then in the digitized image. To monitor saturation a bar chart was produced displaying the saturation levels of the two modelled EMCCDs .

Further characteristics of the fluorophores that can be set include cross-sections and the bleaching decay rates resulting from each illumination field. In the initial implementation, bleaching was modelled as a simple exponential decay. This could empirically be parameterised using the technique presented in section 2.2.2.3. Multiple fluorophores can be established in each location for investigations into the brightness and bleaching rate of protein complexes at a given time in the acquisition sequence. Three images from a simulated acquisition sequence are presented below (Figure 105, Figure 106, and Figure 107). 


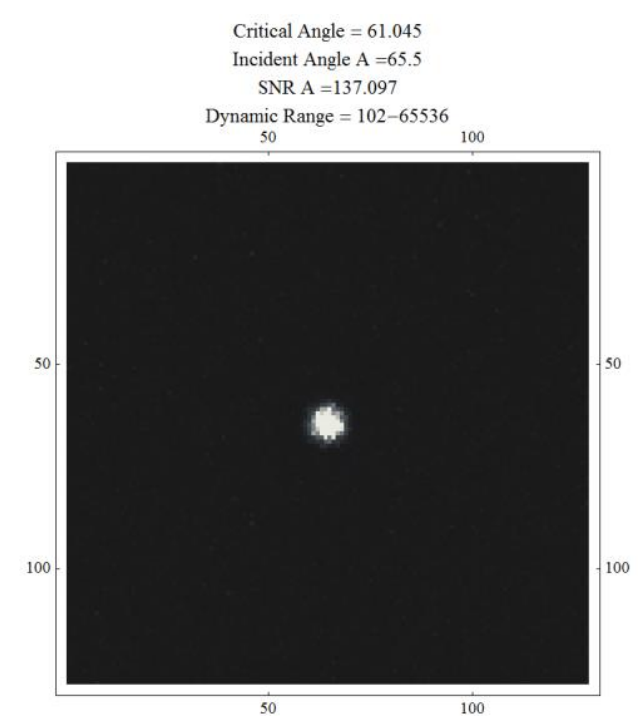

Figure 105 The first frame of a simulated image sequence of 100 colocalised fluorophores. This image illustrates how pixels can be saturated given high enough fluorescence irradiance. Exposure time 0.03 seconds. Values on the $\mathrm{x}$ - and $\mathrm{y}$-axes correspond to pixel columns and rows respectively.

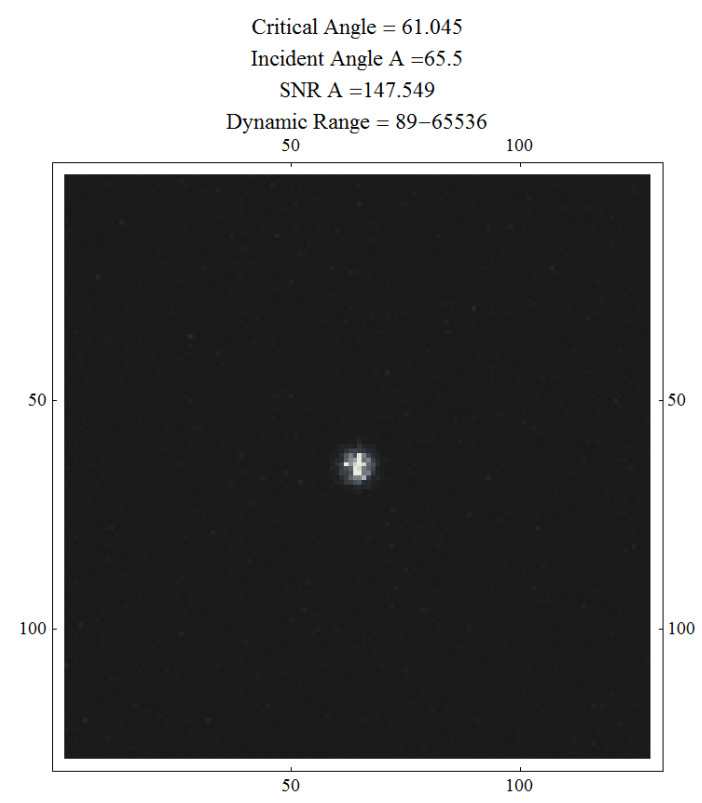

Figure 106 Six seconds later the group of fluorophores has partially bleached due to the illumination light. This can be seen by in the lower number of saturated pixels. Values on the $\mathrm{x}$ - and $\mathrm{y}$-axes correspond to pixel columns and rows respectively. 


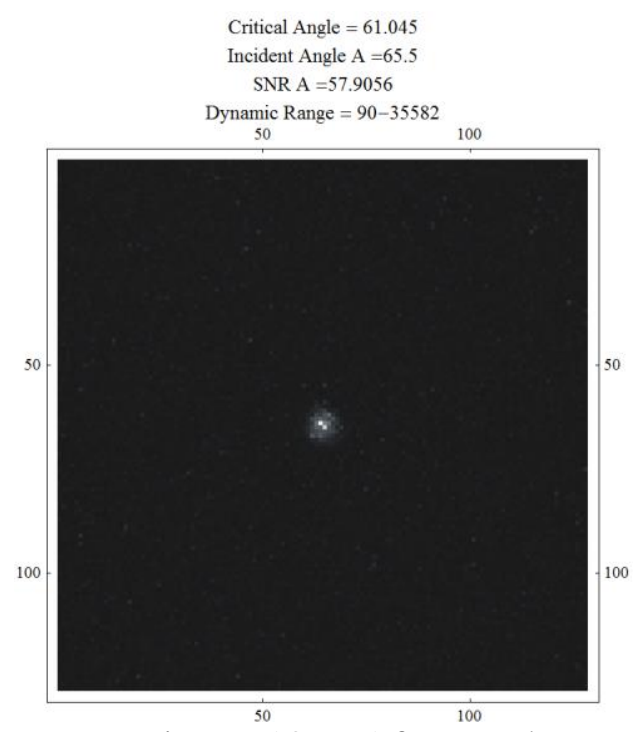

Figure 107 After twelve seconds the fluorescent spot has dropped in intensity further and no saturation is observed. Values on the $\mathrm{x}$ - and $\mathrm{y}$-axes correspond to pixel columns and rows respectively.

The format and functionality of the simulation model was developed and analysis of the model was initiated. However, due to a group decision to aid a fellow student with a further live-cell investigation, the full theoretical analysis and experimental validation of the system model was not completed. The result of this is that though much progress was made in developing a system model, the usefulness of its application was not fully established. The live-cell investigation into which efforts were diverted in presented in section 3.2. 
3 APPLICATIONS OF THE MICROSCOPY SYSTEM 


\subsection{Two-Colour TIRFM to Illuminate the Spatial Organisation OXPHOS Components in $E$. coli}

\subsubsection{INTRODUCTION}

The ability of living organisms to locally create and maintain order distinguishes them in a universe that increases in entropy. The complex molecules of biology are synthesized in anabolic processes using molecules extracted from the environment and broken down in catabolic processes. This metabolic activity fundamentally requires three inputs from the environment; free energy, to drive the decreases in internal entropy, reducing equivalents, donating the electrons of redox reactions that transfer energy, and carbon, the key material component of biological structure. Organisms can be classified by the type of input sources they are able to draw from; light and/or chemical compounds

for free energy, organic and/or inorganic compounds for reducing equivalents, and organic compounds and/or carbon dioxide for carbon.

In all organisms, catabolism produces the cofactor adenosine triphosphate (ATP). ATP is a substrate in signal transduction and the principle molecule for intracellular energy transfer. Of the pathways that release energy to generate ATP, oxidative phosphorylation (OXPHOS) is the most efficient and is widespread. In this process, donor molecules are oxidized by membrane bound enzymes which then pass the electrons to molecules with incrementally lower bond energies. Much of the energy released in this electron transport chain is harnessed to pump protons across the membrane, creating both a potential difference and a $\mathrm{pH}$ gradient. The associated potential energy is released 
when protons pass back through the membrane via the rotary molecule ATP synthase, driving it to reform ATP from adenosine diphosphate (ADP) and inorganic phosphate.

OXPHOS occurs in the inner membrane of mitochondria in eukaryotes and the inner cellular membrane of prokaryotes. Among organisms there can be differences in the structure and constituents of the electron transport chain. Depending on the available types of electron donors and acceptors, bacteria can synthesize suitable pathways from a DNA library containing multiple possible enzymes (Unden 1997). In both E. coli and humans, energy is obtained from chemical compounds and derived electron donors can include NADH and Succinate. In response, very similar enzymes are produced by both species; NADH and Succinate dehydrogenases, and complexes I and II respectively. As well as playing an oxidizing role, NADH dehydrogenase and complex I are trans membrane proton pumps responsible for the electrochemical gradient that somehow drives ATP synthase. The study of the NADH, succinate reductases and ATP synthase in E. coli was therefore seen as potentially informative in understanding of the OXPHOS mechanism and of human biology.

While the individual proteins of Oxidative Phosphorylation have been well characterised (Rich 2003), relatively little is known about their spatial dynamics and organization in living cells. Knowledge of in-cellulo spatial organisation is of relevance to the fields of Bioenergetics and Membrane structure. In Bioenergetics research there is still debate around the mechanism by which the electron transport chain energises ATP synthase (Kell 1992; Donato 2012). The main point of contention is whether, as in Mitchell's Chemiosmotic hypothesis (Mitchell and Moyle 1967), pumped protons that are distributed in the inter-membrane fluid could account for the measured rate of ATP 
synthesis by spatially decoupled ATP synthase. Modifications to the Chemiosmotic hypothesis include models in which pumped protons reside at the membrane fluid interface (James 2012) and localised membrane voltage plays the most significant role (Harvey 2009).

Regarding membrane structure, the trend has been to hypothesize increasing spatial organization and heterogeneity, as seen by research into the possible existence of lipid domains as organizing structures (Karnovsky, Kleinfeld et al. 1982) and the supersession of the "fluid patchwork" (Kusumi, Nakada et al. 2005) over the "fluid mosaic" model (Singer and Nicolson 1972) of membrane structure. Specific to OXPHOS, two basic conceivable alternatives are that OXPHOS complexes traverse the membrane independently or that they exist as function-specific super complexes (Lenaz and Genova 2007). Super complexes of electron transport components have been conceived as functionally advantageous (Dudkina, Kouřil et al. 2010) and native gel electrophoresis has revealed such complexes in yeast and mammalian mitochondria (SchaÈgger and Pfeiffer 2000). In vivo imaging in my group led to the "Respirazone" hypothesis, proposing that within E. coli, OXPHOS enzymes colocalise within mobile function-specific membrane patches (Lenn, Leake et al. 2008).

In this new investigation, I set out to falsify the respirazone hypothesis and complement my group's previous highly quantitative analyses of OXPHOS proteins (Lenn, Leake et al. 2008). Limited by the photophysical characteristics of the fluorescent proteins expressed in the available strains, there remains a difficulty in obtaining compelling high contrast images of OXPHOS protein localisation. The strategy taken in this investigation was therefore to focus on the single question of whether or not 
functionally related proteins are colocalised in the inner membrane. All aspects of the experiment were tailored to produce clear images to indicate the spatial distribution of the proteins of interest. This investigation explored ways in which the optimal experimental settings could be determined. It was decided that an attempt should be made to optimise the experiment at a system-level and a combination of traditional trial and improvement and a model-based approach was used (Harriman and Leake. 2013).

Previous works have utilised E. coli strains developed through lambda redmediated recombination (Pohl, Uhlmann et al. 2007) by my collaborator Heiko Erhardt in which a range of OXPHOS proteins are expressed as Fluorescent protein-fusion constructs. This genomic encoding approach has been shown to produce fluorescentlytagged protein at levels which are comparable to the unmodified cell strains. This contrasts with the more common approach of plasmid expression that would produce significantly more protein than would occur naturally. Due to the size of the FP tag being comparable to that of the protein under study there will be some impairment of functionality. In this case, the fluorescent proteins were found to not dramatically effect cell growth and OXPHOS (paper submitted). For this investigation a subset of the strains was selected corresponding to the specific proteins of interest. Double-labelled strains expressing GFP-NuoF/mCherry-SdhC and mCherry-NuoF/ATPase-GFP were investigated along with the parental strain (BW25113) and strains expressing FP-fusion with each protein of interest individually. Details of the chemical activity and fluorescent labelling of these proteins are presented below in Figure 108. Research in the submitted article found that each of the selected proteins existed in intracellular complexes containing multiple copies. In the dual-label strains complexes of NADH dehydrogenase 
I, Succinate dehydrogenase, and $\mathrm{F}_{1} \mathrm{~F}_{0} \mathrm{ATP}$ synthase were found to include 10-20, 20-40, and 40-60 molecules per spot respectively. Analysis of mobility of the spots in all strains found that roughly half exhibited anomalous/sub-diffusive behaviour, and the remainder exhibited Brownian diffusion at a mean rate of $0.007 \pm 0.005 \mu \mathrm{m}^{2} \mathrm{~s}^{-1}( \pm \mathrm{SD})$. No significant dependence was observed on either diffusion coefficient or confinement radius with number of fluorescent proteins in each spot.

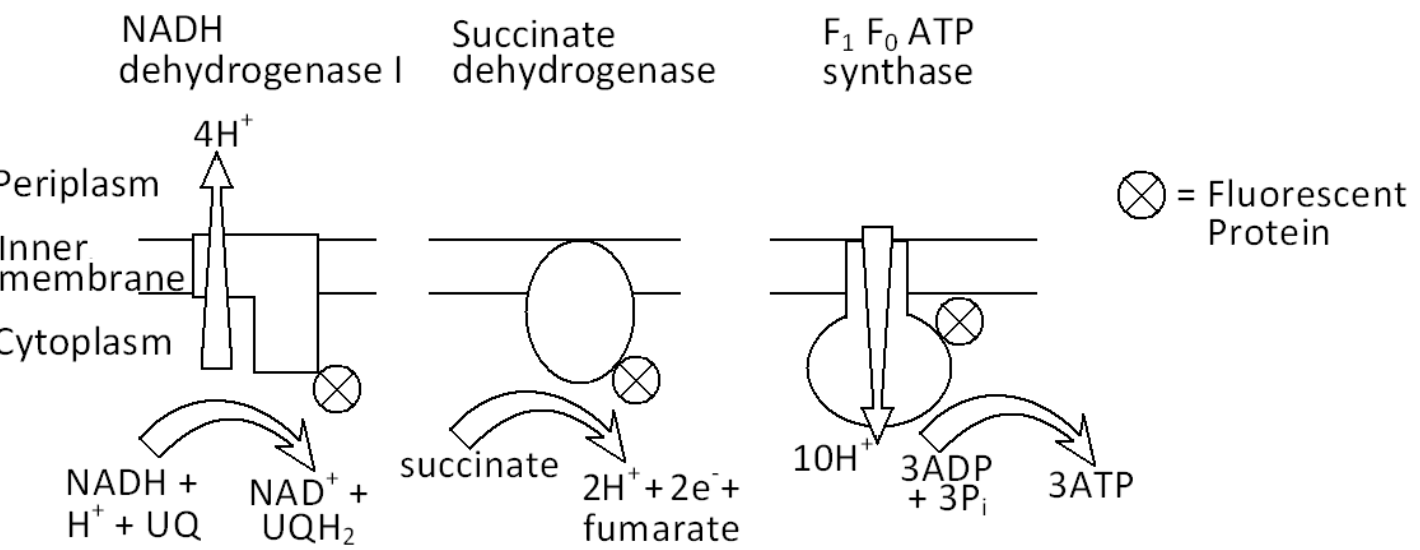

Figure 108 Schematic depicting the OXPHOS proteins under investigation with details of chemical activity and approximate positions of fluorescent labels.

\subsubsection{MATERIALS AND METHODS}

\subsubsection{Experimental Procedure}

E. coli cells were grown in lysogeny broth (LB media) for 3.5 hours at 30 degrees $\mathrm{C}$ and shaken at $120 \mathrm{rpm}$ reaching OD600nm of $\sim 0.02$. To increase the cell density, the sample was centrifuged to pellet the cells ( $5500 \mathrm{x}$, $2 \mathrm{~min}$ ), $95 \%$ of the supernatant was 
removed, and the cell pellet was re-suspended in the remaining $5 \%$ of media. A $3 \mu 1$ sample of the re-suspended cells was applied to a thin agarose gel (1\% agarose in M63 media) and mounted on a microscope slide with a plastic support. The gel was allowed to dry, immobilising the cells within the agarose matrix, and a coverslip was applied to the surface of the gel. The slide was then viewed under the microscope. Growth and imaging conditions were the same for all strains and all imaging was completed within 1.5 hours of removal of cells from the incubator. From testing of numerous cleaning techniques, plasma cleaning was found to be necessary in removal of all fluorescent contamination.

Unlike in previous studies, I used a stage-top incubator to set a temperature of $37^{\circ} \mathrm{C}$ as in the lower intestines, the native environment of E. coli. Imaging was carried out with the bespoke fluorescence microscope build from a Nikon inverted Eclipse model using a 1.49 NA oil immersion objective lens. Image acquisition was achieved with a cooled Andor iXonEM+860 $128^{2}$ pixel EMCCD camera, giving a field of view 8.8 microns across. Brightfield illumination was produced with a halogen lamp and since use of the stage top incubator precluded use of a high NA condenser lens; a custom condenser lens was installed to achieve high enough flux through the sample for brightfield imaging from a much greater working distance.

The TIRF illumination was used in order to preferentially illuminate the cell membrane. Excitation was achieved with $473 \mathrm{~nm}$ and $561 \mathrm{~nm}$ light and an illumination path designed to enable precise and independent control of the incoming beam angle of incidence, intensity and diameter. In the emission path the mCherry and GFP fluorescence emissions were split using dichroic filters and imaged on adjacent regions of 
an EMCCD. An adjustable rectangular aperture was placed in an intermediate image plane in order to restrict the extent of the projected images to half of the EMCCD each.

Prior to cell imaging precise co-focussing and approximate alignment of the emission channels was carried out using $80 \mathrm{~nm}$ latex beads in brightfield. In cell imaging, the brightfield illumination mode was used to locate cells in the sample prior to switching to the TIRF mode for image acquisition. The $473 \mathrm{~nm}$ and $561 \mathrm{~nm}$ excitation beams were set to input angles that would result in penetration depths of $100 \mathrm{~nm}$. The sample was rastered in a consistent direction perpendicular to the incident laser illumination in order to not image any cell more than once. Triggering of the camera and illumination shutters was achieved with purpose written LabVIEW code and imaging details were set through the proprietary camera software. The full pixel array was used to acquire 16Bit video images with an electron multiplying gain setting of 300, and 60 milliseconds exposure time.

Bleedthrough is the proportion of fluorescence intended for one colour channel that passes through filters into the other colour channel. If the bleedthrough is high it can result in seeing the same feature in both channels and mistakenly perceiving the colocalisation of two different types of fluorescent molecule. The filters used in the microscope were selected by using the system model and specifications from filter manufacturers. With the selected emission path filters, the bleedthrough was predicted using the system model and determined experimentally by imaging high concentrations of fluorescent protein in solution. The green-to-red bleed through was measured at $\sim 5 \%$ while the prediction from simulation was $10 \%$. From red-to-green, the bleedthrough was measured as $<1 \%$ and had been predicted to be $5 \times 10^{-7} \%$. 


\subsubsection{Generation of a Spatial Transform for Equating Positions in the Red and Green Images}

Due to the projection of the green and red channel images onto adjacent regions of the EMCCD it was necessary to measure the actual positions of respective image features in each channel. With this information a geometric transform was computed and applied to the red channel half of all images to enable precise relation between images in the two channels. It was necessary to prepare a sample with objects that would appear in the images of both channels and be localised with high precision. With diffraction limited fluorescent features this could in theory be done with arbitrary colocalisation precision (Thompson 2002). Orange fluorescent Latex beads of $100 \mathrm{~nm}$ diameter were immobilised on the surface of cover glass with poly-L-lysine and images were taken of frames with three or more beads distributed evenly in the field of view. Five separate videos were taken and transforms were computed from each. The results were inspected to identify correct algorithmic object identification and a correct transforms were selected. This process was repeated after live-cell imaging and comparison of the transforms from before and after enabled verification that the channels images had not been displaced on the EMCCD array during the experiment. 


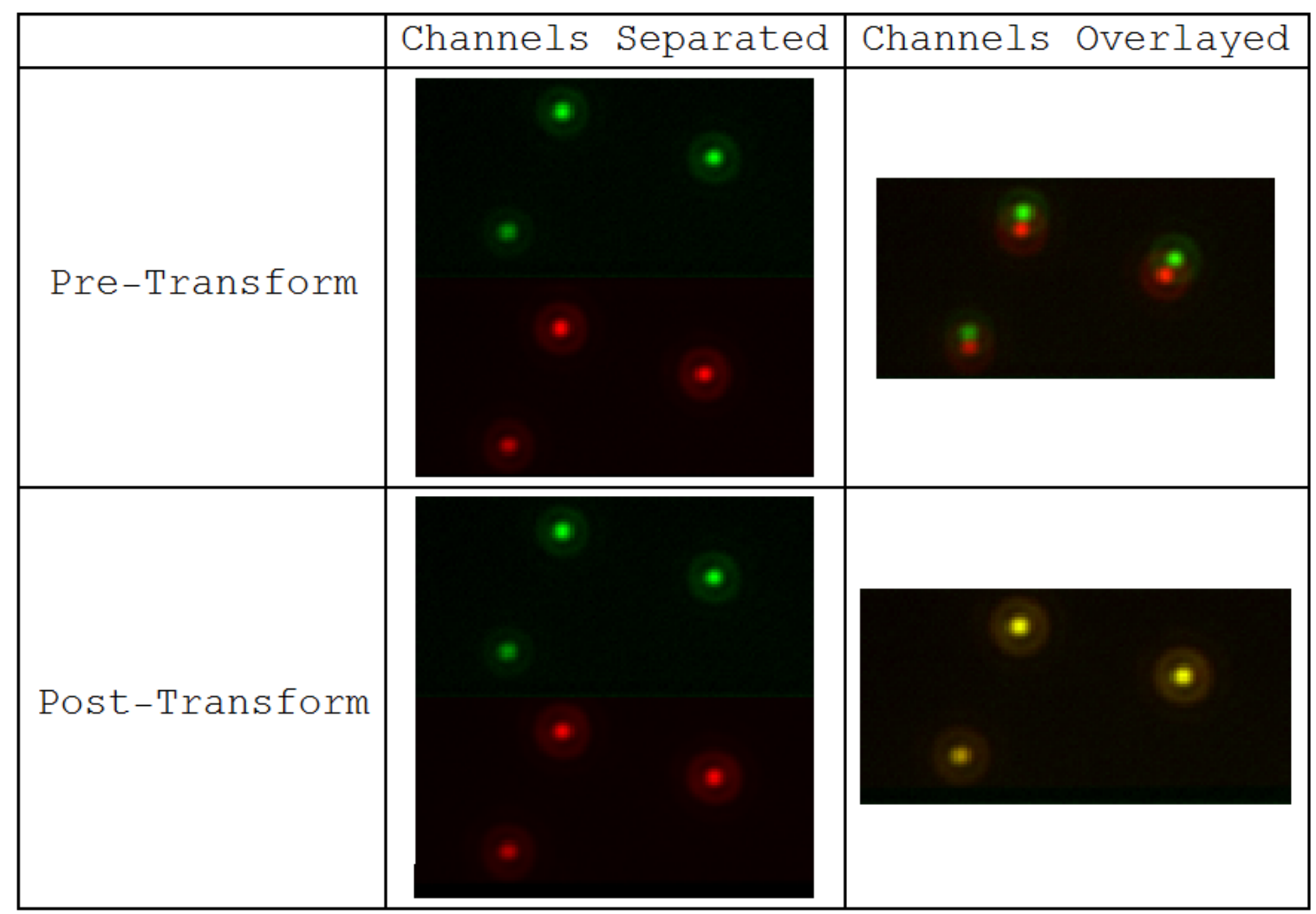

Figure 109 Figure illustrating the difference in images on the red and green sections of the EMCCD array and the application of a spatial transform to correct the difference.

\subsubsection{Optimising Imaging Parameters}

In the effort to optimise imaging conditions, it was necessary to carefully define acceptable parameter boundaries based on the information required from the samples under investigation. For example, a suitable exposure time was determined through imaging cells at a range of exposure times and identifying the video in which fluorescent features had the highest contrast. Upon increasing the exposure time the SNR eventually dropped due to the effect of blurring resulting from the mobility of the fluorescence. 
Acquiring images at $60 \mathrm{~ms}$ is advantageous over acquiring at $30 \mathrm{~ms}$ and averaging every two frames due to the lower read noise contribution.

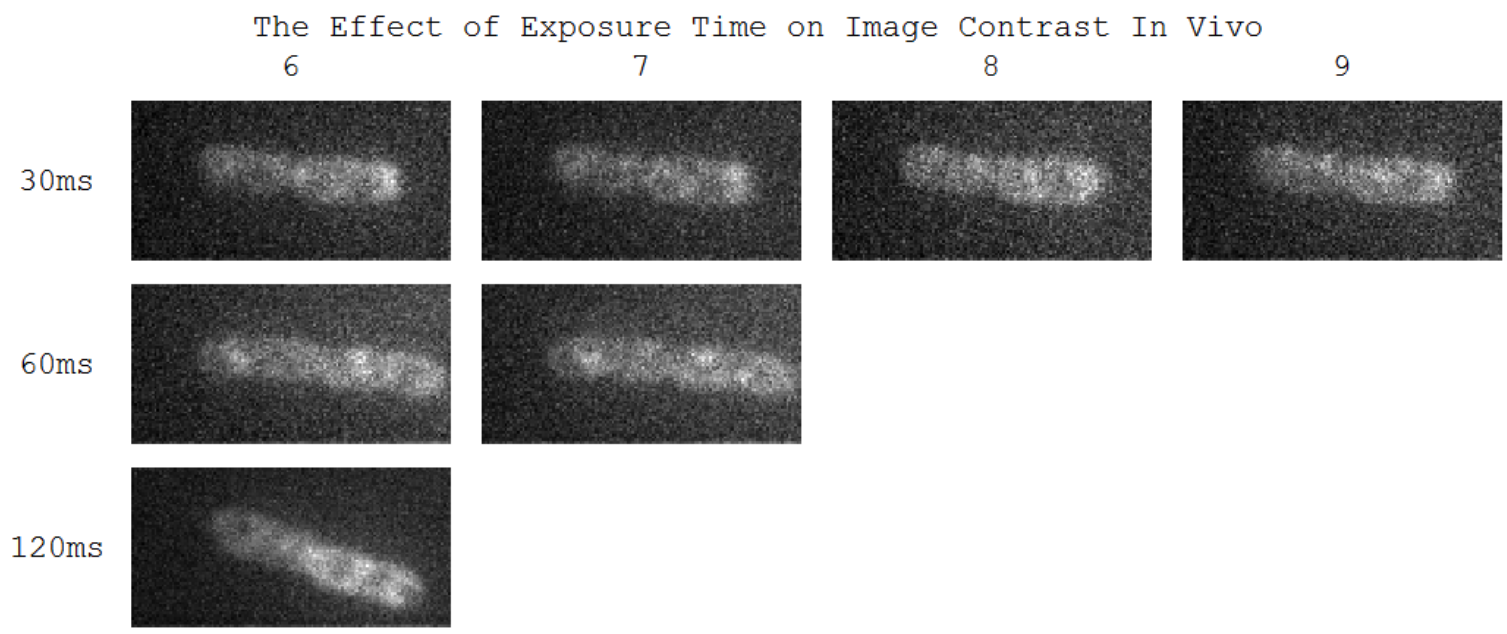

Figure 110 An exposure time of 60ms was chosen to obtain a high SNR and to sample the mobile fluorescence at a high enough rate. Though $30 \mathrm{~ms}$ is the suggested exposure time for capturing molecule dynamics (Kusumi, Nakada et al. 2005) the SNR was considered too low given the goal of producing high contrast images. At $120 \mathrm{~ms}$ the contrast was high but the location of molecules became ambiguous.

Many other parameters affected the video suitability including properties of the illumination such as irradiance of the sample and settings of the EMCCD. Laser powers were tuned in order to lengthen and equalise the bleaching rates of the fluorescent proteins in the cells. To obtain the highest SNR for a given exposure time, the EMCCD pixels should be almost saturated and the highest bit depth and EM gain should be used. Fortunately this was possible given the images resulting from the exposure time and the laser powers applied. Once good imaging parameters had been determined for live-cell imaging it was necessary to ascertain whether the images could account for all fluorescent proteins present, not just regions of high intensity where multiple fluorescent 
proteins reside. Images were therefore taken of single Fluorescent proteins immobilised to a coverslip surface and it was confirmed that single-molecule sensitivity was indeed possible.

\subsubsection{ResUlts}

\subsubsection{Sub-Sampling of Data}

Ten videos of each cell type were taken with identical imaging conditions. Features were evident in the images of labelled channels most of the time but not always. This was attributed to either a genuine diffuse fluorescence in cells or loss of imaging focus. Since where features were visible it was known that the focus was correct, only videos with clear features were selected for analysis. This meant that the results of this investigation can only be open-ended in the sense that they might assert that various possibilities have been observed, but not that they are the only possibilities. In the videos of each strain, distinct mobile features were evident for around twenty frames. The second five frames of each video were selected for analysis as they were found to exhibit the clearest cellular features. The first five frames exhibited the highest signal, but were neglected due to the very rapid drop in absolute intensity and a relative homogeneity of the image over their time period. 


\subsubsection{Distinguishing Signal and Noise}

\subsection{Distinguishing Cell Types through Total Cell Intensity}

It was hoped that each cell type could be identified by its total intensity in each channel. An algorithm was written to generate masks to allow for automated cell image analysis. The masks were produced from binarisations of the first frame of each fluorescence video since the cell image was of the highest intensity relative to the background and typically homogeneous. Erosion was also applied to ensure the mask did not include any background pixels. The total intensity of each cell in each channel in the first frame of imaging is presented in the scatter plot below.

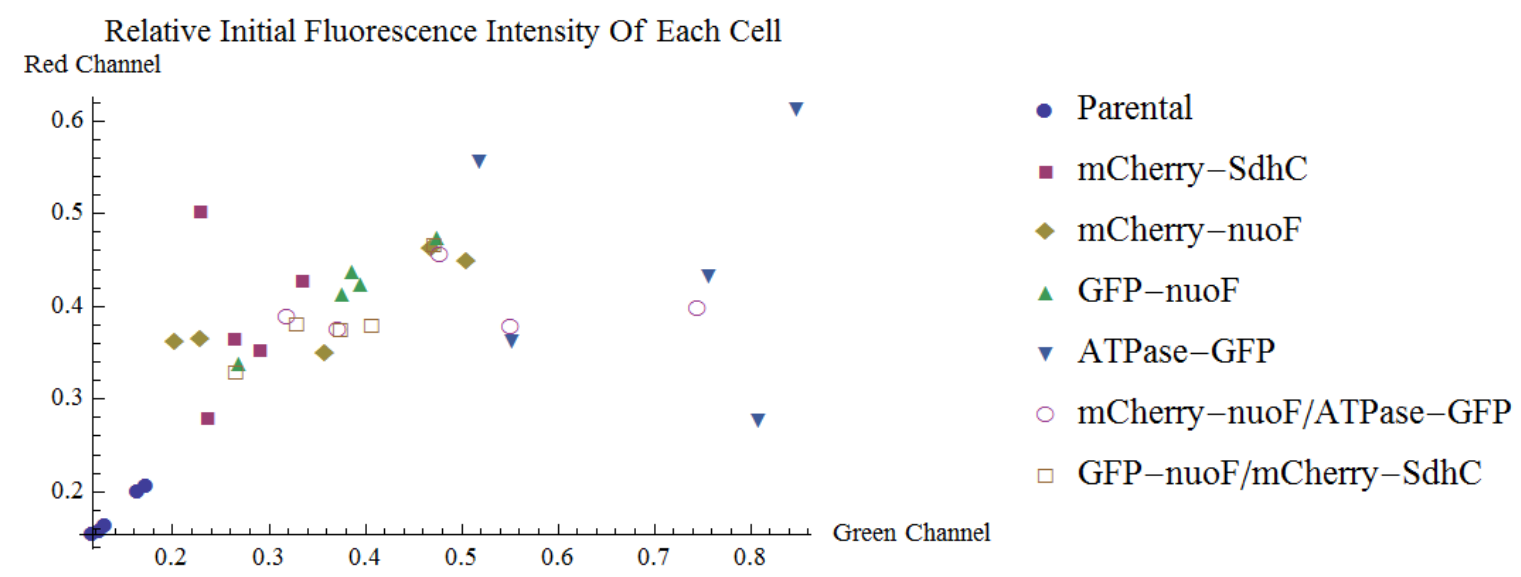

Figure 111 This plot shows the relative total pixel intensity of each cell in the initial frame of each video. Heterogeneity of expression level across the imaged cells is evident.

Cells of the parental strain are located in the plot where one would expect, with lower intensities in both channels. This demonstrated that parental and labelled cells could be distinguished by their total intensity. However, the labelled cells were not 
distributed as one would expect. With ideal labelling and a perfectly suited imaging system one would expect a scatter plot in which the points would fit into one of four quadrants based on whether they were labelled or not in each channel.

The cells cannot be divided this way and there are counterintuitive cases where a cell's expressed GFP emits more strongly in the red channel than cells expressing mCherry and vice versa. The measured bleedthroughs of 5\% from GFP into the red channel and $<1 \%$ from mCherry into the green channel are unable to account for this. The dramatic variation in whole-cell initial intensity indicates either varying expression levels or varying levels of pre-bleaching prior to imaging. Effort was made to reduce inadvertent pre-bleaching of cells through the imaging protocol. The sample stage was scanned in a consistent direction orthogonal to incident beam of illumination. If imaged cells had been inadvertently bleached by the illumination for previously imaged cells one would expect a trend in reduced initial cell intensity with video number. No such trend was observed suggesting indirect pre-bleaching is unlikely to have occurred.

\subsection{Distinguishing Cell Types through Inspection}

The analysis and conclusions of the investigation were dependent on the ability to discern (from the data, not from knowledge of the cell type) whether a fluorescence feature observed in a cell was due to signal or noise. As seen in the cell intensity scatter plot (Figure 111), due to the unpredictability of bulk cell intensities, a consideration of the spatial features of fluorescence would be necessary. Only if features due to labelling could be identified should colocalisation analysis be believed. For example one would 
need to be able to determine whether the "colocalisation" seen was colocalisation of GFP and mCherry or GFP and autofluorescence. The effectiveness of a technique for distinguishing features of signal and noise could be tested by comparing the results it gained on many cells with the knowledge of the cell types analysed.

Typically in single-molecule studies, tracking algorithms are applied that fit a two-dimensional Gaussian to spots and information pertaining to the molecule is extracted. This approach is particularly useful in cases where accessing information on molecular mobility and stoichiometry are experimental goals. As previously stated, the scope of this experiment was limited specifically to addressing the question of colocalisation. To this end, a relatively long exposure time was used that precluded the possibility of tracking due to the irregular fluorescent spots resulting from their acceleration. The regions of fluorescence were still evident from inspection and it was desired that the human visual system be put to as effective use as possible in image analysis.

Well known problems exist in the visual interpretation of images. Primarily, any analysis will be a subjective interpretation of the images and will be susceptible to a diversity of predictable and unpredictable biases. Quantitative information is not attainable, but due to its high processing power, inspection is key in validating the results of all quantitative approaches. A pervasive practice in the collection of subjective data is the use of double blind studies. Inspired by this, an approach was devised to reduce the susceptibility of image interpretations to prior knowledge (and the resulting expectations/desires) of the strains. A specific example of bias would be that if one expected to see colocalisation then one might be more inclined to perceive colocalisation. 
Code was written that would isolate every frame of interest (of all the videos of all the strains) and randomize their order. Each image would then be presented in this random sequence and predetermined multiple choices was selected from. A user input was made to select whether the cell was labelled with nothing, with GFP, with mCherry, or with both (Figure 112). The selections were stored with their respective frames and the frames re-ordered into their original sequence and attributed to the correct strain type. The selected interpretations were then compared against what was known to be true (based on knowledge of the strains) and statistics were computed (Figure 113). This method was used to establish whether the presence of signal could be correctly discerned through visual inspection. The data showed that the number of selections of each labelling configuration was proportionate to the actual number of labelling configurations. This was important since if one always made the same selection one would obtain $100 \%$ success for a strain with that configuration and the result could be misinterpreted as a good ability to discern fluorescence labelling. 


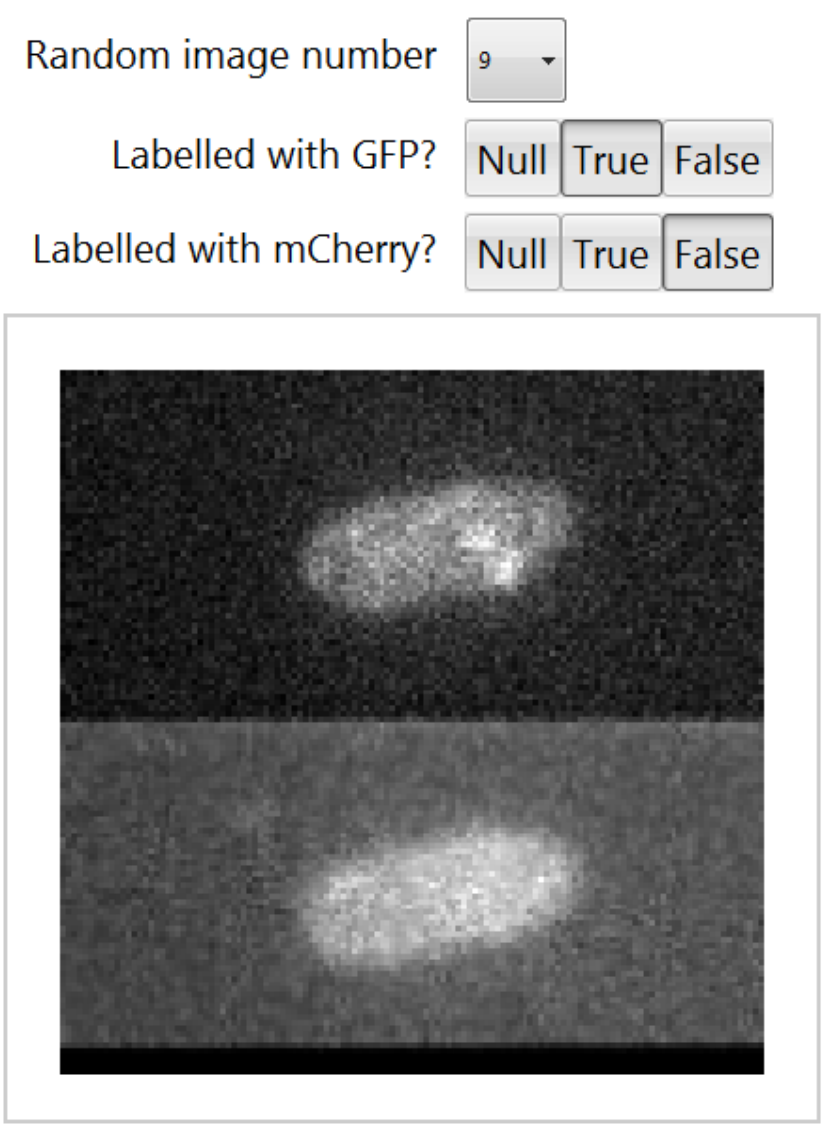

Figure 112 Example screen shot of graphical user interface of program used to determine how accurately one could visually distinguish cell types based on apparent labelling in the images.

\begin{tabular}{|c|c|}
\hline Cell strain & Label Identification Accuracy (\%) \\
\hline Parental & 100 \\
\hline mCherry - NuoF / ATPase - GFP & 82 \\
\hline GFP - NuoF / mCherry - SdhC & 54 \\
\hline GFP - NuoF & 74 \\
\hline mCherry - SdhC & 92 \\
\hline mCherry - NuoF & 82 \\
\hline ATPase - GFP & 78 \\
\hline
\end{tabular}

Figure 113 Summary of the results of random cell identification tests. Accuracy was computed as the percentage of images for which the cell labelling was correctly identified. 
The final results show a high success rate in visually discerning the labelling of each strain except for in the case of the GFP-nuoF/mCherry-sdhC strain. A degree of caution was hence associated with all analysis results of the GFP-nuoF/mCherry-sdhC strain. Details of the results showed a greater success rate in identification of the labelling in the green channel than in the red channel. This could be explained by the higher noise level in the red channel resulting from direct excitation in the red channel. The detailed results further showed a higher likelihood of making the correct selection when a label was present than when not present. This makes sense since labelling will produce high contrast features while in a channel with an absence of labelling one may incorrectly perceive features due to noise.

\subsubsection{Computation of Fluorescence Correlation}

\subsection{Low pass Spatial Filtering of Images}

Each raw image exhibits a grainy quality resulting from the noise contributions. These include Shot Noise associated with low photon intensity and Read Noise associated with the EMCCD. As is seen in images with a high SNR (for example the orange beads of Figure 109), the graininess is smoothed out. The smallest features visible in a high SNR image are those corresponding to the point spread function of the imaging system. This feature of image formation was used to clarify what image features could be attributed to the sample fluorescence (as opposed to noise). A low pass spatial filter was applied to the raw images to remove the image features smaller than the

microscope PSF. The effect of this spatial filtering can be seen in Figure 114 below. The 
low pass filter used was the general "LowpassFilter" function in the Mathematica computing environment. The input arguments were the image data and a cut-off frequency. The cut-off frequency was determined through its effect on the image of a point source. The frequency was selected such that the output was a spot of the same radius as the PSF measured in experimental images. The effect of the low pass filter on a simulated point source can be seen in the top two images of Figure 114 below. The effect of the same filtering on an experimental image of a cell can be seen in the bottom images of Figure 114.
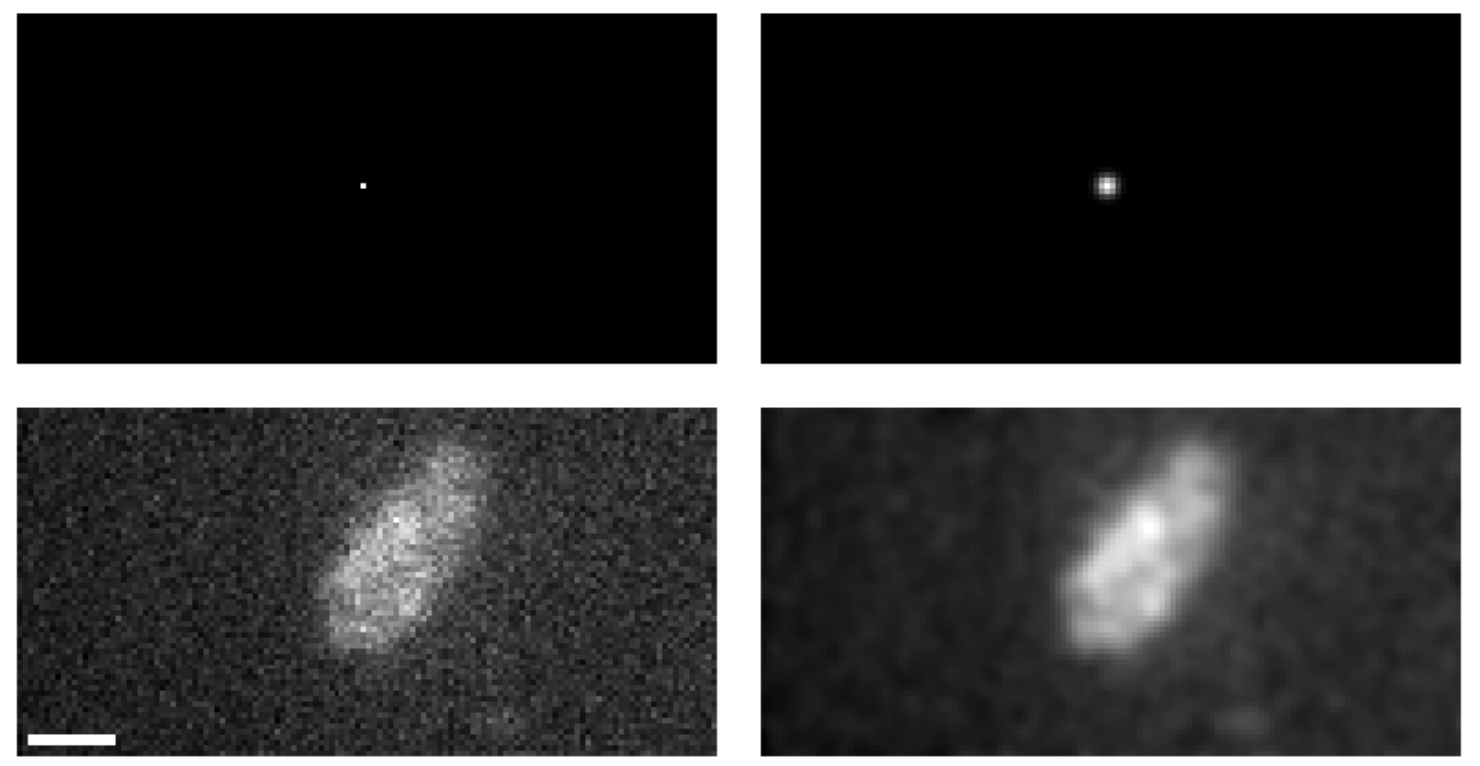

Figure 114 Top left, a simulated point source; top right, the effect of applying a lowpass filter to the simulated point source. Bottom left, an experimental image of a cell; bottom right, the effect of applying the same lowpass filter to the experimental cell image. Scale bar 1 micron. 


\subsection{Colour Combination for Maps of Channel Correlation}

To enable comparison of all cell images their intensity ranges were rescaled to be from zero to one. This step resulted in a loss of information on absolute intensity, but enabled comparison of feature location in images with differing total intensities. To produce initial insight into spatial correlation, the channels of each (monochromatic) frame were combined into an RGB image. As well as the intensity variation present in the monochromatic images, these images included the dimension of hue. The channel correlation at each pixel could be identified by the hue resulting from mixing of the green and red components. Maximal likelihood of colocalisation is indicated by bright yellow as seen in the top right corner of Figure 115 in which a range of red and green values are presented with the result of their mixing.

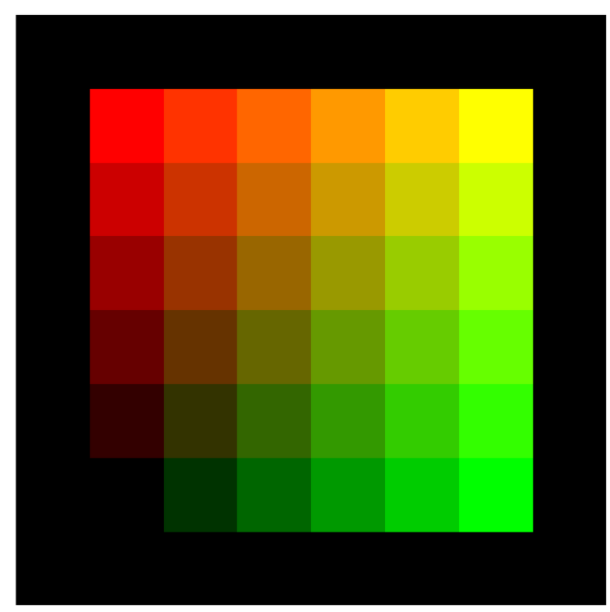

Figure 115 The two dimensional colour scale, RGB values $(0,0.2,0.4,0.6,0.8$, and 1), of green and red and the resulting mixed colours (black background for contrast).

In Figure 116 a sample of the RGB cell images are presented. Inspecting the RGB images of the double-labelled strains, one could see clear regions of both high 
correlation (colocalisation) and low correlation. This led to the first conclusion of the investigation, that it is not strictly the case that the molecules of interest are always colocalised in the membrane. Since examples of both colocalisation and anticolocalisation were apparent in individual frames, the rest of the analysis was aimed to determine whether the frequency of colocalisation was greater than would be expected from chance interaction of molecules following independent trajectories.

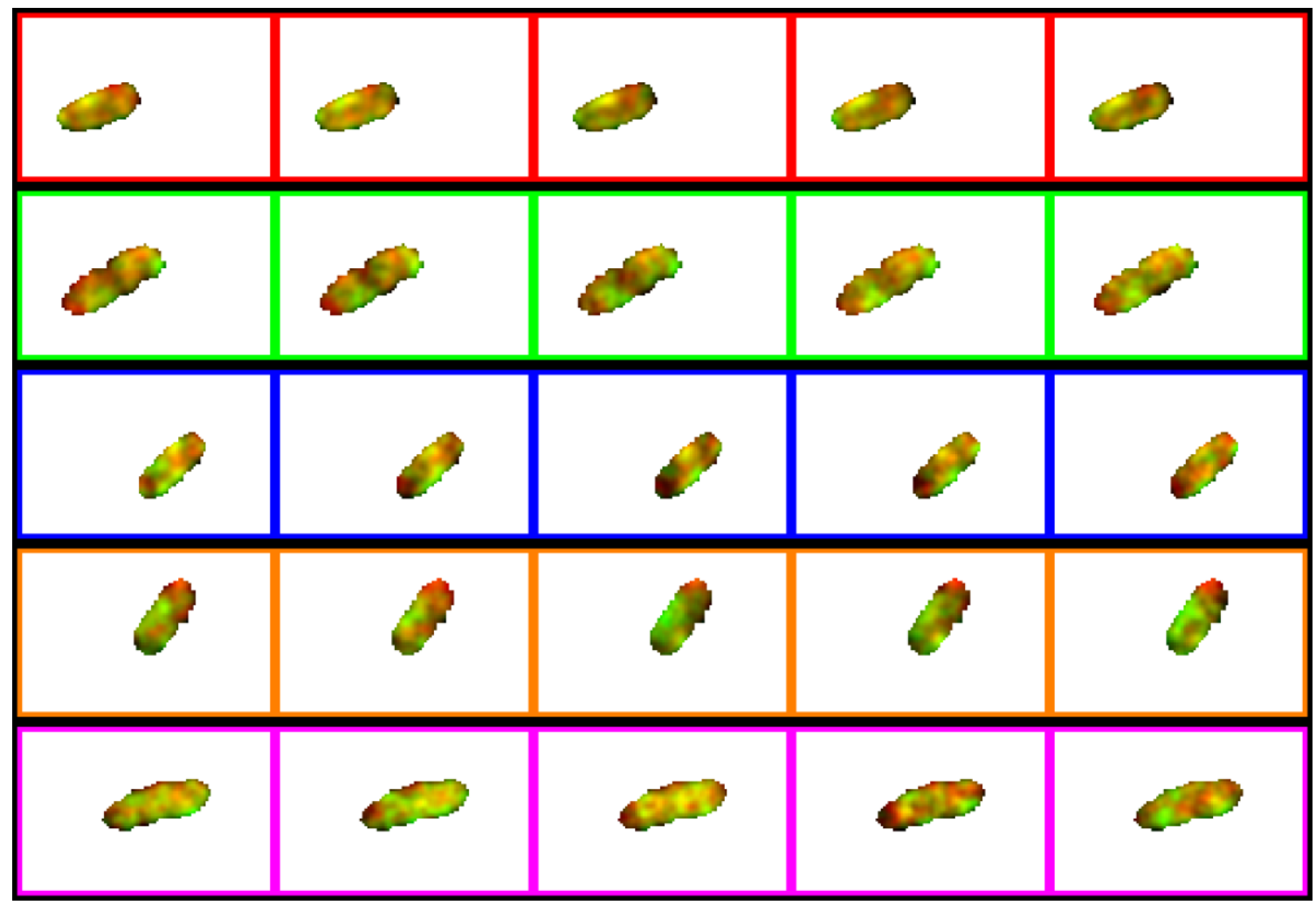

Figure 116 Combined images of the GFP-nuoF/mCherry-sdhC strain. 


\subsection{A Quantitative Measure of Correlation}

While retaining all information of relative pixels values within each cell image, the combined colour images are difficult to interpret due to the multiple gradations in hue and intensity. A single quantitative metric for correlation was desired. Sophisticated statistical measures of correlation exist such as Pearson's correlation coefficient and Spearman's rank correlation coefficient (French, Mills et al. 2008), and with further techniques the precision of colocalisation can be accurately calculated (Koyama-Honda, Ritchie et al. 2005). In this investigation a relatively simple approach was developed that was nonetheless seen to be suitably informative. The highest value pixels in each cell were distinguished from the rest (Figure 117 left). The images could then be compared based on the proportion of overlap between the high value pixels (Figure 117 right). The binarisation threshold was set as the upper quartile pixel value within each cell image. This resulted in binary images with $25 \%$ of the pixels in each cell having the high value. When the cell images of each channel were overlaid, colocalisation would be identified through the location and total number of overlapping high-value pixels. This approach lost information on relative pixel intensity, but could produce a single and consistent metric on cell correlation. 

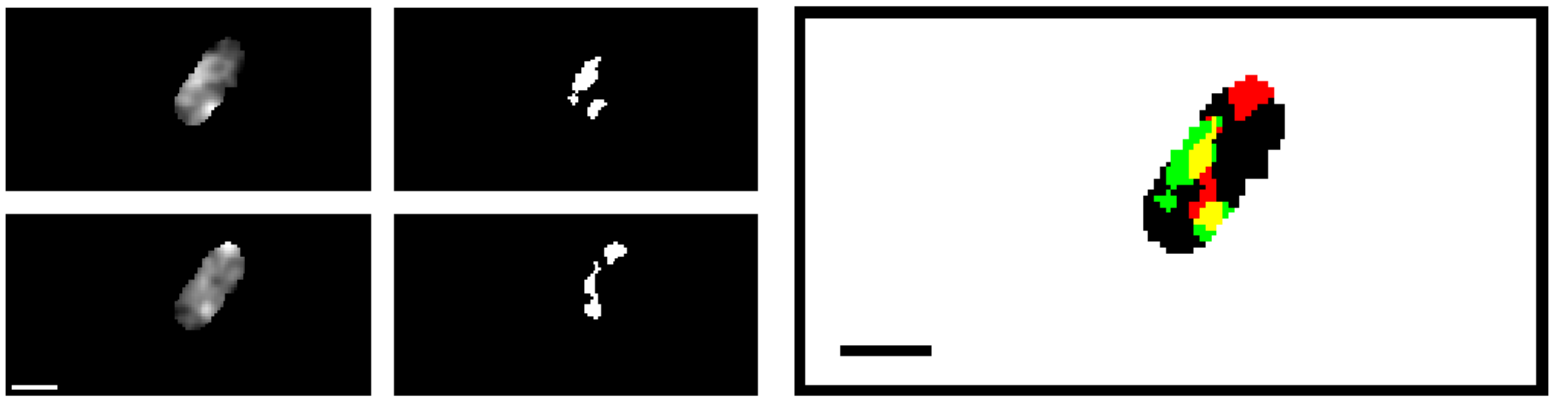

Figure 117 From a frame of the IIV strain. Left) Filtered Green (top) and Red (bottom) channel images of a masked cell. Centre the images binarised to leave $25 \%$ of the pixels as high valued. Right) The combined image with colocalisation evident in yellow. In this image, regions of both colocalisation and anti-colocalisation are evident. Scale bar $1 \mu \mathrm{m}$.

\subsection{Simulated Random cells}

When taking a theoretical approach to evaluation of colocalisation, there is a difficulty is in saying what constitutes random localisation. This is due to the effects of molecular mobility, bleaching, cell curvature, and a basic lack of knowledge of the state of the specific membrane. By assuming there to be an equal likelihood of each pixel having a high value in the binarised images, one can make an approximation of the proportion of cell pixels that would be colocalised due to independent molecular motion. Since a quarter of the pixels in each cell are green and a quarter are red, a random distribution of each colour would result in a probability of $(1 / 4 \times 1 / 4) 1 / 16$ colocalisation. The values of colocalisation were plotted against time as percentages of the maximum correlation possible, the most likely value corresponding to $25 \%$. In reality it is inadequate to assume there to be an equal likelihood of cell pixels having a high value in the binarised images. 
A simulation was made as a benchmark for visualisation and analysis of a cell in which the pixel values of each channel were randomly distributed and independent. Videos were generated of this simulated random cell for analysis and comparison with the videos of live strains. The frames of the simulated random strain videos can be seen in the first row of Figure 118.

\subsection{Fluorescence Correlation}

A value for spatial correlation was determined for each frame of each video of each strain (including five random cell simulations) (Figure 118). The correlation results for each strain are averaged and presented in Figure 119.

Figure 118 Figure spread over the following two pages. Left) The RGB combination of the binarised red and green images for each frame. Right) Plots for each strain of the correlation computed for each frame of each video against the time of the frame exposure (in milliseconds following the opening of the illumination shutter). The image frames and corresponding correlation curves are colour coded. Each image is framed with a colour specific to the video sequence to which it belongs. The corresponding correlation values are plotted on the adjacent graphs and the curves are

colour coded accordingly. The first row shows the simulated random strain data and analyses, and the following rows show real experimental data and analyses. 


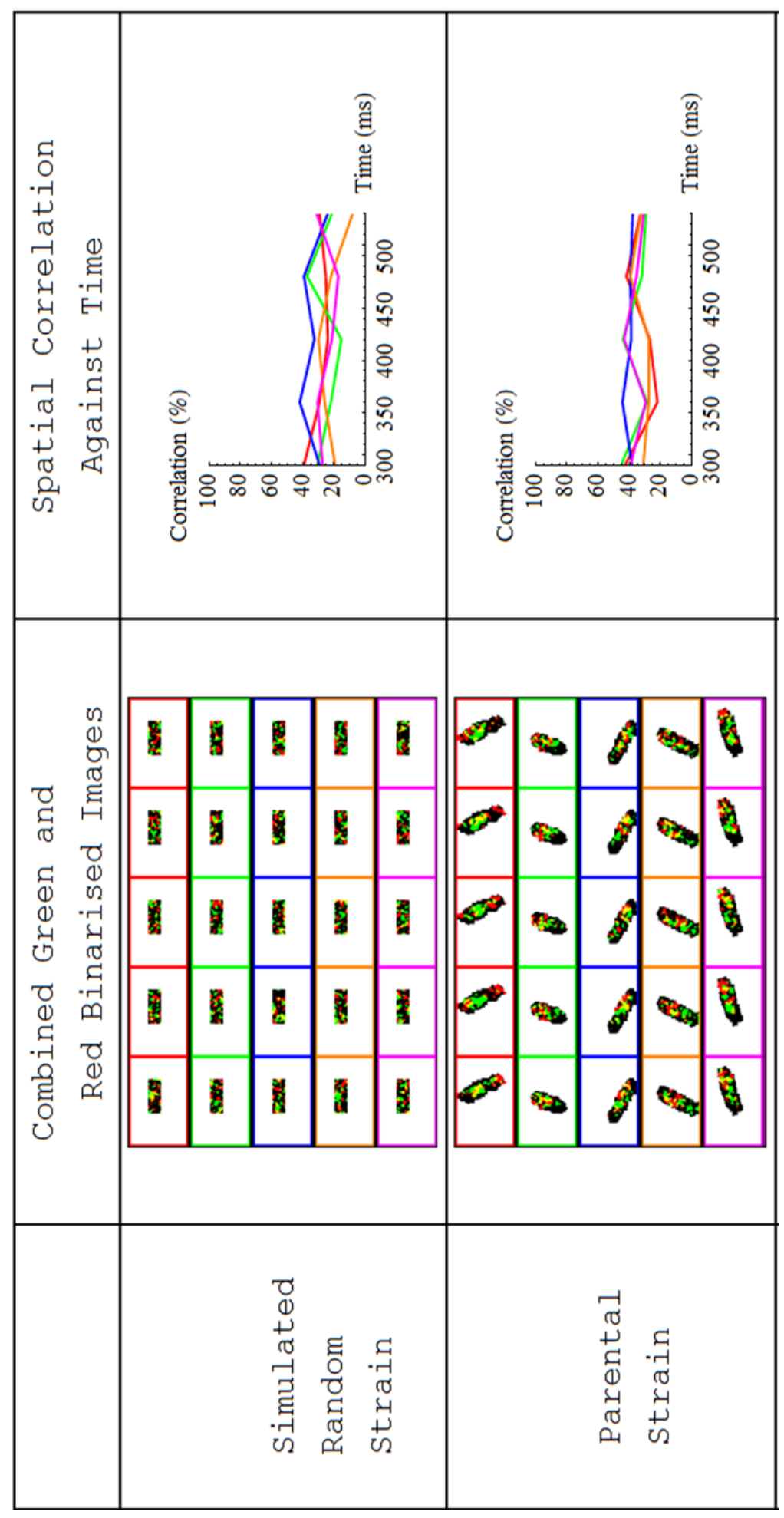




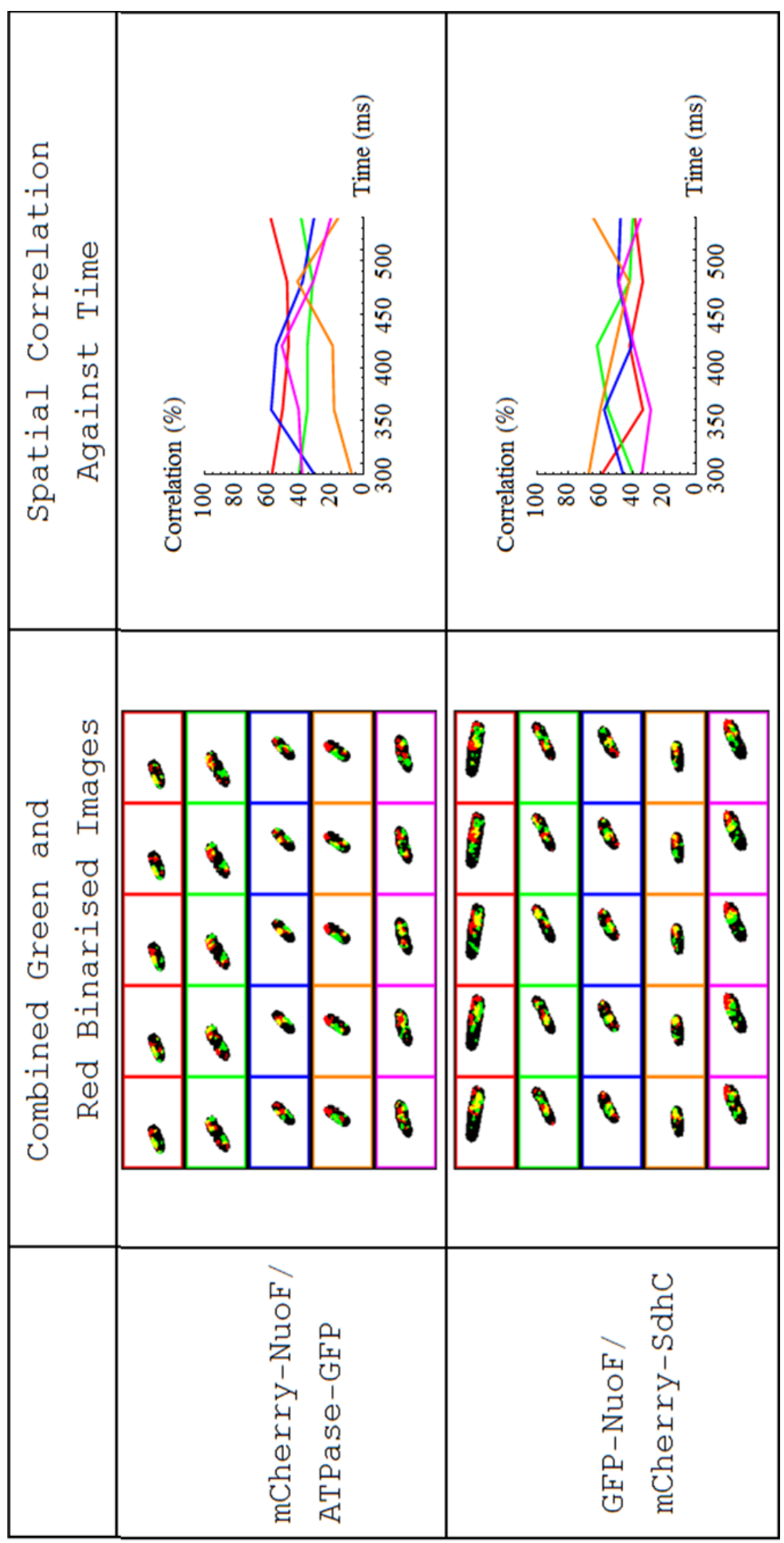


Correlation (\%)

\section{Average Spatial Correlation of Each Strain}

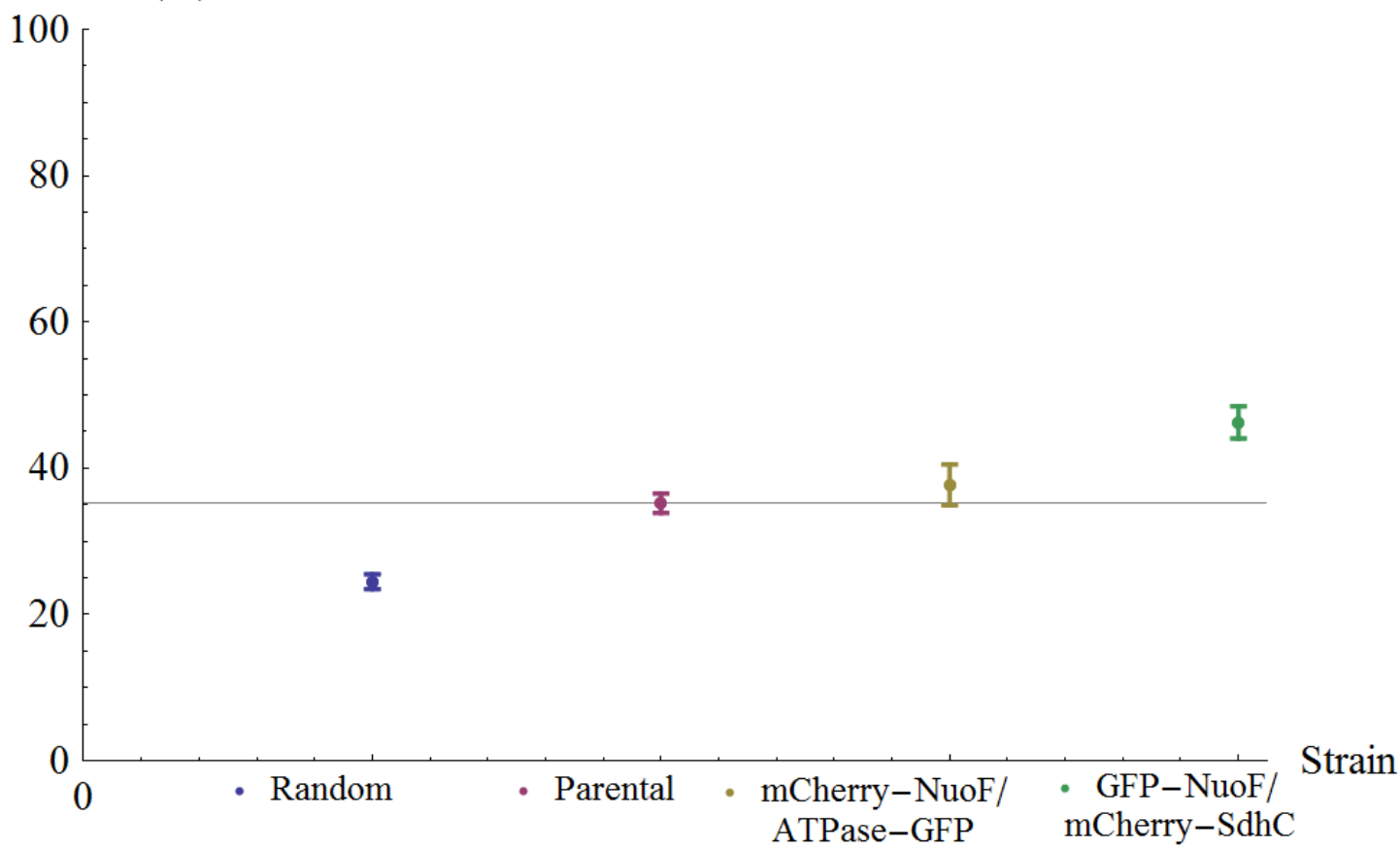

Figure 119 For each strain the average correlation value over all videos and strains is plotted. Error bars correspond to \pm the standard error of the mean, indicating the probability that actual mean lies where it is marked.

The horizontal gridline in Figure 119 indicates that the only comparable results are those of the parental strain and the mCherry-NuoF/ATPase-GFP strain. This means it is not possible to assert that there is significant colocalisation of NDHC dehydrogenase I and $\mathrm{F}_{1} \mathrm{~F}_{0} \mathrm{ATP}$ synthase. The standard error bars of the GFP-NuoF/mCherry-SdhC strain do not overlap with those of the other strains, suggesting that there is significantly more correlation of NDHC dehydrogenase I and Succinate dehydrogenase. This lends support to the hypothesis that electron transporters may exist, transiently, in functionally linked complexes. 


\subsection{Summation of Binarised Images}

Summing the five selected frames can be compared to a long exposure time image in which the fluorescent features are blurred due to their mobility. In these images, the brightness of a pixel corresponds to the number of times that it had a high value attributed to it. This type of image is illustrative of the mobility of the fluorescence and of cellular regions with consistently high fluorescence. When these images are combined into RGB images they produce very compelling maps of fluorescence correlation. It should be noted though that regions of bright yellow indicate a high likelihood of colocalisation, but do not necessitate it because the effective exposure time is five times longer than in the individual frames and dynamic information is lost.

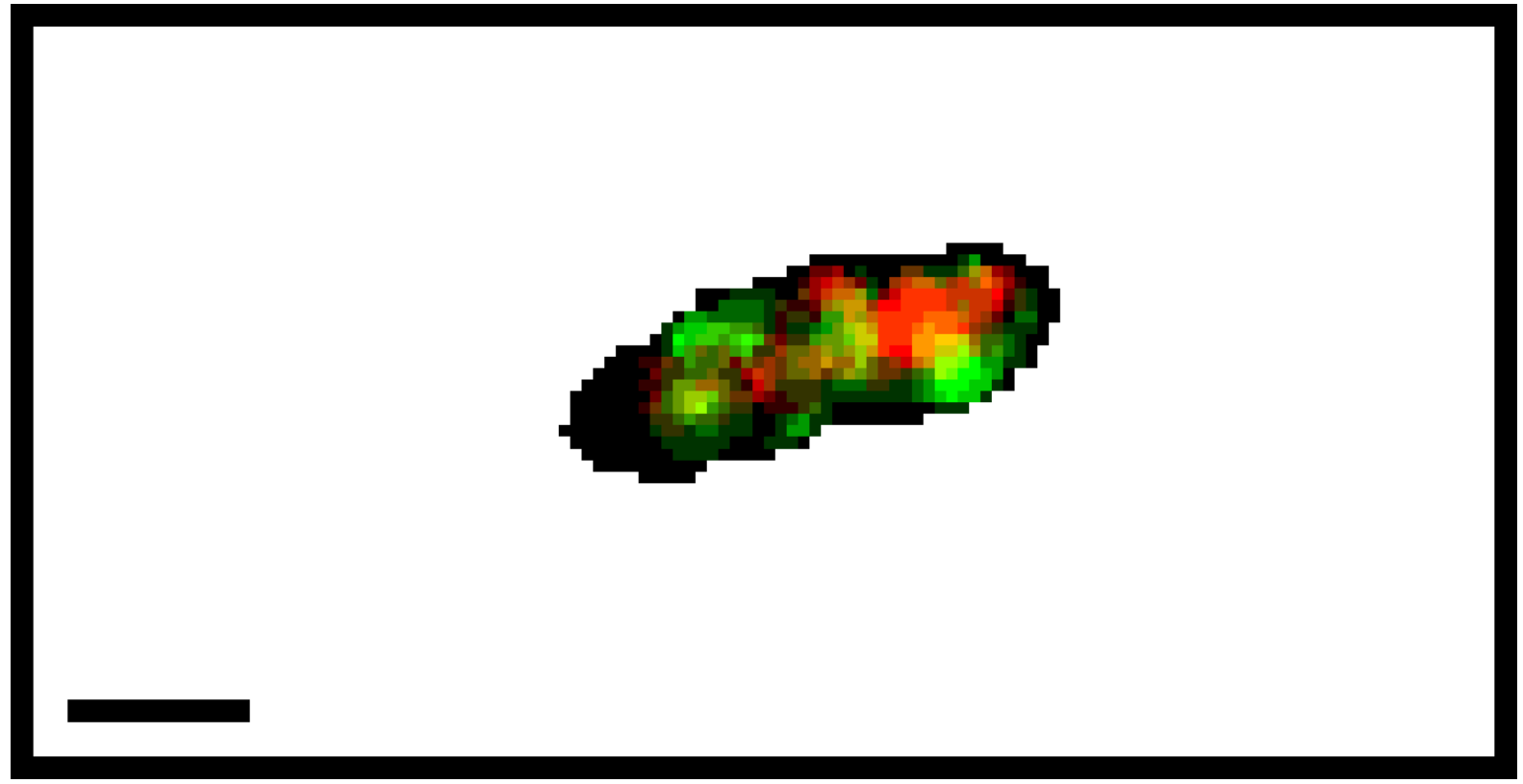

Figure 120 Summed image of all frames in a video. Scale bar $1 \mu \mathrm{m}$. 


\subsection{Novel Correlation Analysis Technique}

A technique for analysis of fluorescence correlation was developed with the aim of exploiting intracellular molecular mobility. The technique was based on the realisation that in past and future frames, "realistically redistributed" fluorescence is observable. So as well as comparing the channel images of one frame, channel images should be compared between frames from different times. The knowledge of what pseudo-independent (time displaced) colocalisation looks like can then be used in the analysis of the colocalisation in a single frame. This technique produces information on the motion of fluorescence from frame to frame which is used in interpretation of the correlation information.

For a given video, either a channel is evaluated against itself (green-green or redred correlation) for information on realistic localisation and mobility, or one channel is evaluated against the other (green-red correlation) for information on molecular correlation. To explain the technique, a grid can be formed in which the rows and columns are associated with the sequence of frames of the two channels under analysis. For example, the rows (top to bottom) could be associated with the frames of the green channel and columns (left to right) the frames of the red. The correlation of images associated with the row and column for each position in the grid is calculated. This means that a correlation measure is determined not only for images taken at the same time point, but also between frames displaced in time. The hue of each element signifies the colour channels that have been compared, and the brightness indicates the correlation value. 
To infer a high likelihood of colocalisation, values along the main diagonal are compared to the rest. If brighter than average, this indicates that the spatial distribution of intensity in both channels is correlated. If the intensity can be shown to be from the fluorescently labelled proteins, this indicates colocalisation of the labelled molecules. There is total colocalisation when one channel is compared with itself (as in Figure 121 left and centre) where the diagonal elements have the maximum brightness. This is not necessarily the case when images of different channels are compared (as in Figure 121 right).
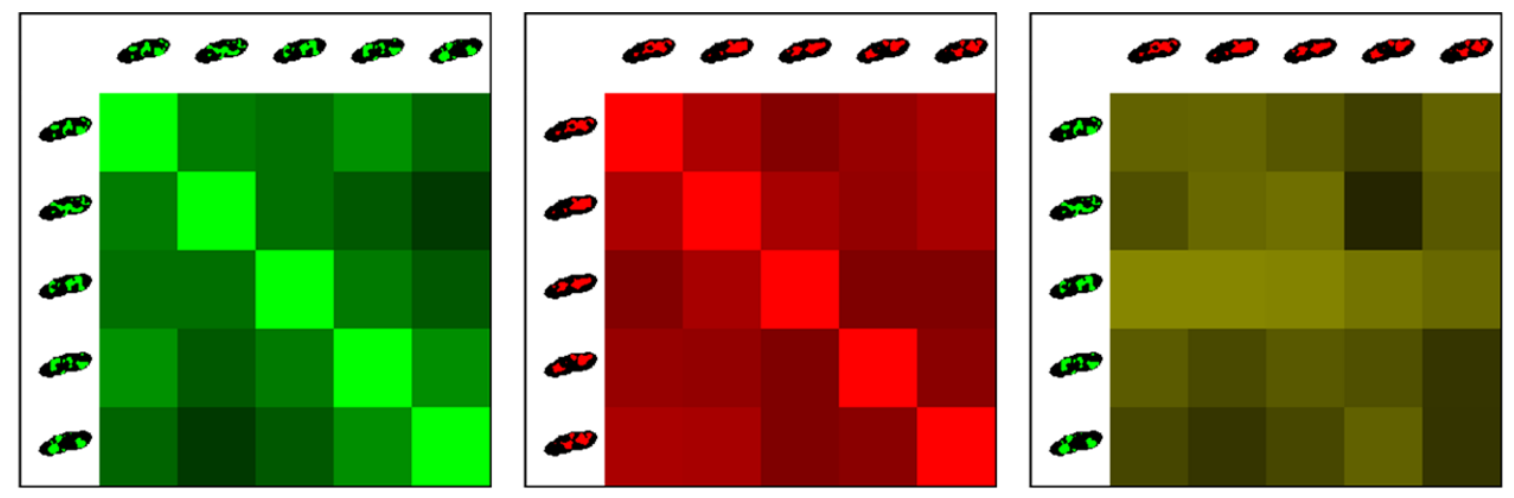

Figure 121 Arrays illustrating level of correlation between each frame of five frame video sequences. (Left) Green-green correlation, (centre) red-red correlation, and (right) green-red correlation. Brighter colour corresponds to higher correlation.

All videos were analysed as above and statistics were generated relating the level of colocalisation to the displacement in time of the compared frames. The time "displacement" being the modulus of the difference between the start times of two exposures. Each diagonal of the grids (as in Figure 121) can be seen to contain element values corresponding to a specific time displacement. The average element values for each time displacement were plotted. Error bar heights were based on the standard 
deviation of the respective element values. Examples of such plots for the single video in Figure 121 and the average values for all videos of the respective strain are presented below (Figure 122). All data was analysed with this method and is presented in Appendix 5.1.3.

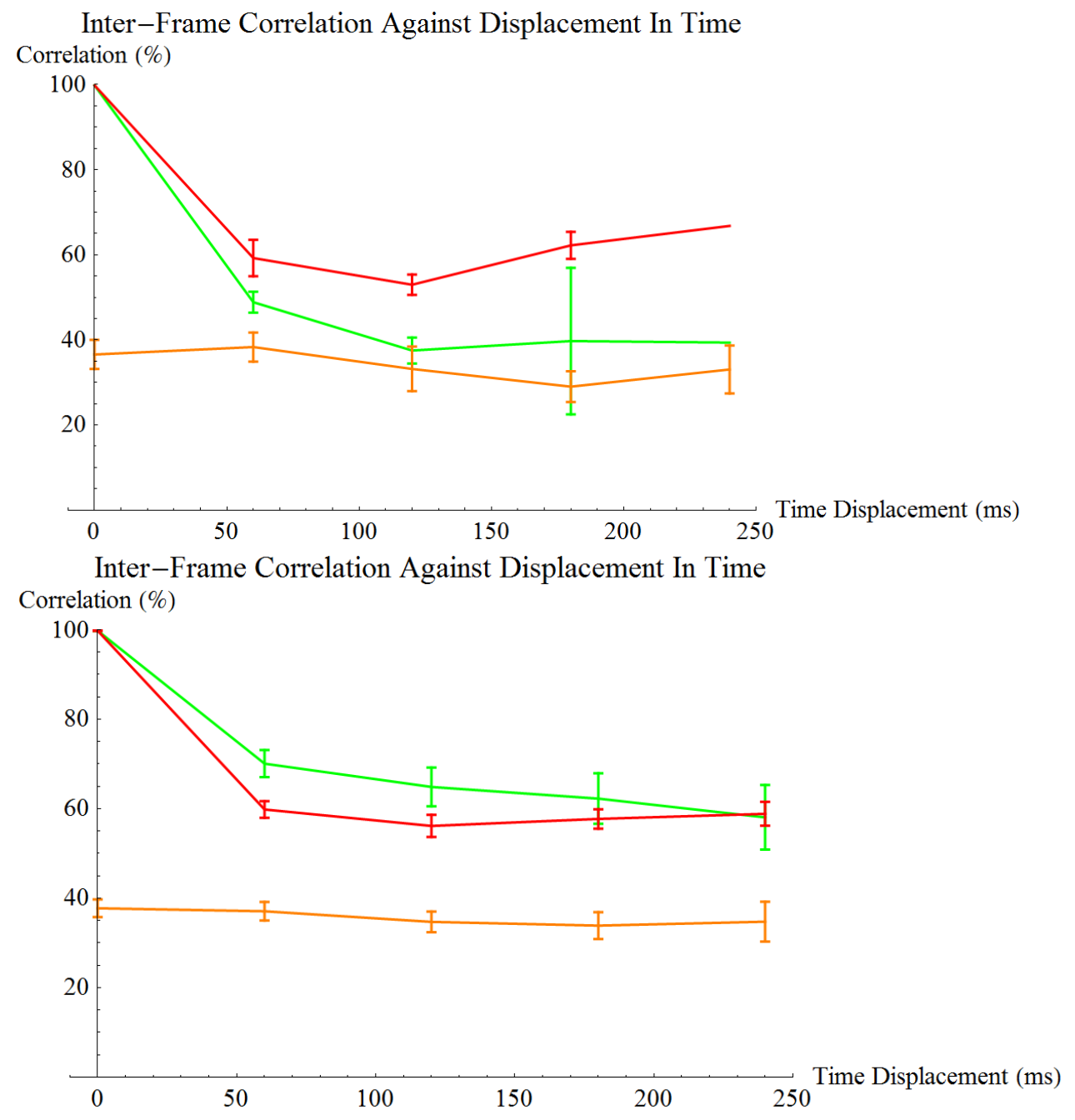

Figure 122 Correlation against frame time displacement plots for the data presented in Figure 121 (top) and (bottom) the average of all videos of the same strain (the mCherry-nuoF/ATPase-GFP strain). These plots show the change in image correlation as the difference in capture time of the compared images increases. The 
colours indicate the channels of the images being compared. The green and red curves corespond to correlation of the green and red channel images with themselves. The orange curve coresponds to the correlation of the green and red channels with each other. The error bars show \pm the standard error of the mean for each time displacement.

The green-green and red-red time displacement data indicates the level of decorrelation due to noise, and the mobility and spatial extent of each fluorescent component. Regardless of contents of the channel there will be $100 \%$ correlation at zero time displacement. After one frame time displacement all the de-correlation due to noise will have occurred. If combining the data for all the videos of a strain, the curve would ultimately become flat. The ultimate correlation value would be indicative of whether the fluorescent species were constrained within a sub region of the cell. As discussed before, for total spatial de-correlation one would expect a value of $25 \%$ in the long term. Decorrelation beyond the time displacement of one frame would occur on a timescale dictated by the mobility of the fluorescent species the cell. Whether this de-correlation is observed depends on whether the exposure time of the video frames is short enough to capture the change.

The green-red time displacement data would indicate whether the fluorescent components were colocalised. At zero time displacement the noise contributions would be de-correlated (unlike in the green-green and red-red data) and the correlation level would be the time-average measure of fluorescent correlation. The gradient of the curve from zero time displacement would indicate whether the fluorescent signals were colocalised or not. A gradient of zero would indicate un-correlated fluorescence; a negative slope would indicate fluorescent colocalisation. If green and red fluorescent species followed independent trajectories but were both constrained within the same sub 
region of the membrane, the zero time displacement correlation would be higher than if they were unconstrained. In the two exceptional cases where fluorescent species are constrained to be totally spatially separated or totally colocalised, the correlation would not change with time displacement. These cases would be identified in the images.

Inspection of Figure 122 (bottom) reveals that, for the mCherry-nuoF/ATPaseGFP strain, the final average value of green-green or red-red correlation is higher than the green-red correlation. This is due to limited movement of the fluorescent features over the period of the five frames. This immobility is evident in the summed images. It can be seen however that the slope continues downwards as the regions of fluorescence move over a long timescale. In the equivalent plot for the simulated random strain all average values become totally de-correlated within one frame Figure 123.

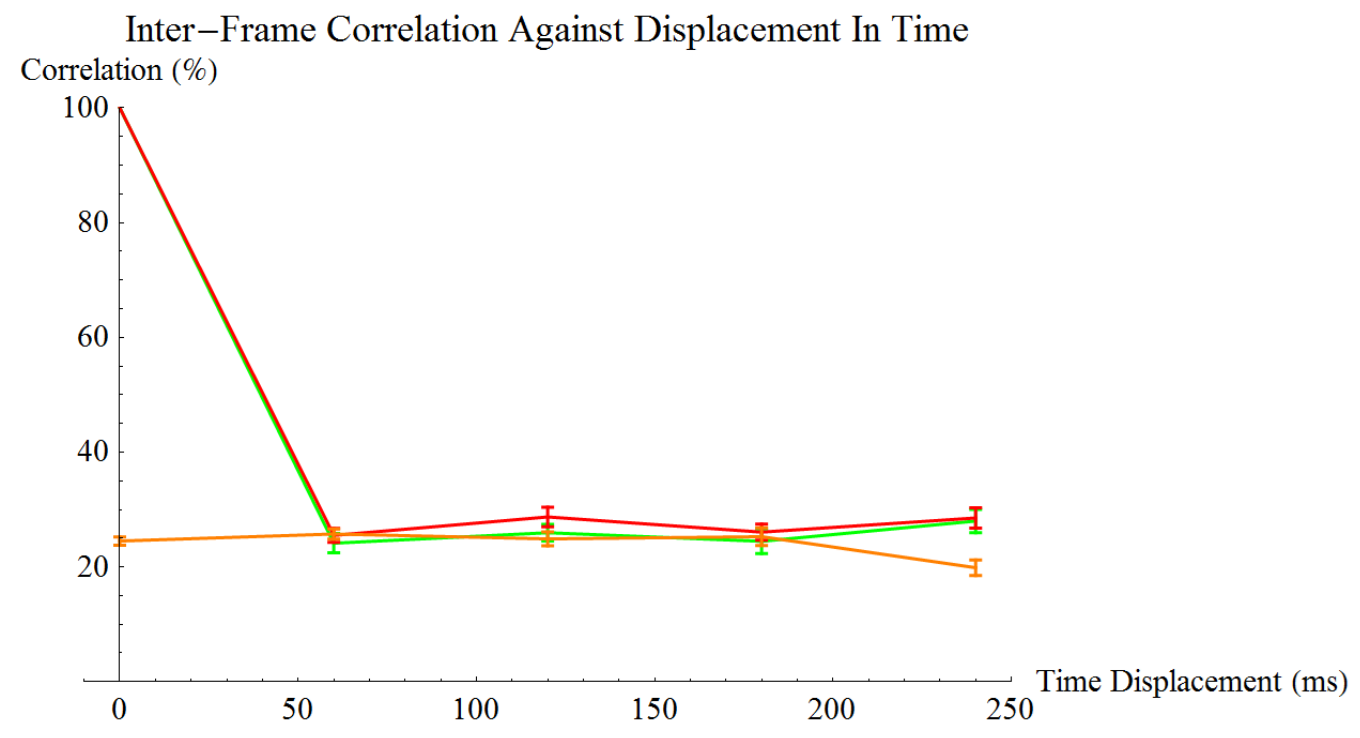

Figure 123 Correlation against frame time displacement plot for the average of all videos of the simulated random strain (as seen in the first row of Figure 118). These plots show the change in image correlation as the difference in capture time of the 
compared images increases. The colours indicate the channels of the images being compared. The green and red curves corespond to correlation of the green and red channel images with themselves. The orange curve coresponds to the correlation of the green and red channels with each other. The error bars show \pm the standard error of the mean for each time displacement.

\subsection{Correlation of Red and Green Channel Displaced in Time}

Figure 124 displays the average correlation values against time displacement for each strain. When the data is averaged for each strain, no clear change in correlation with time displacement is observable. For each curve the error bars mostly overlap and there is no significant downward trend. It is therefore not possible to assert from this analysis, though it is not ruled out, that there is colocalisation in either of the double-labelled strains. As expected, the correlation of simulated random data hovers around the $25 \%$ mark. From this plot the GFP-nuoF/mCherry-sdhC strain maintains a significantly higher correlation than the mCherry-nuoF/ATPase-GFP strain. Given its high level of correlation at zero time displacement, one would have expected the GFP-nuoF/mCherrysdhC correlation to drop with time displacement to ultimately equal that of the mCherrynuoF/ATPase-GFP strain. 
Inter-Frame Spatial Correlation Against Displacement In Time Correlation $(\%)$

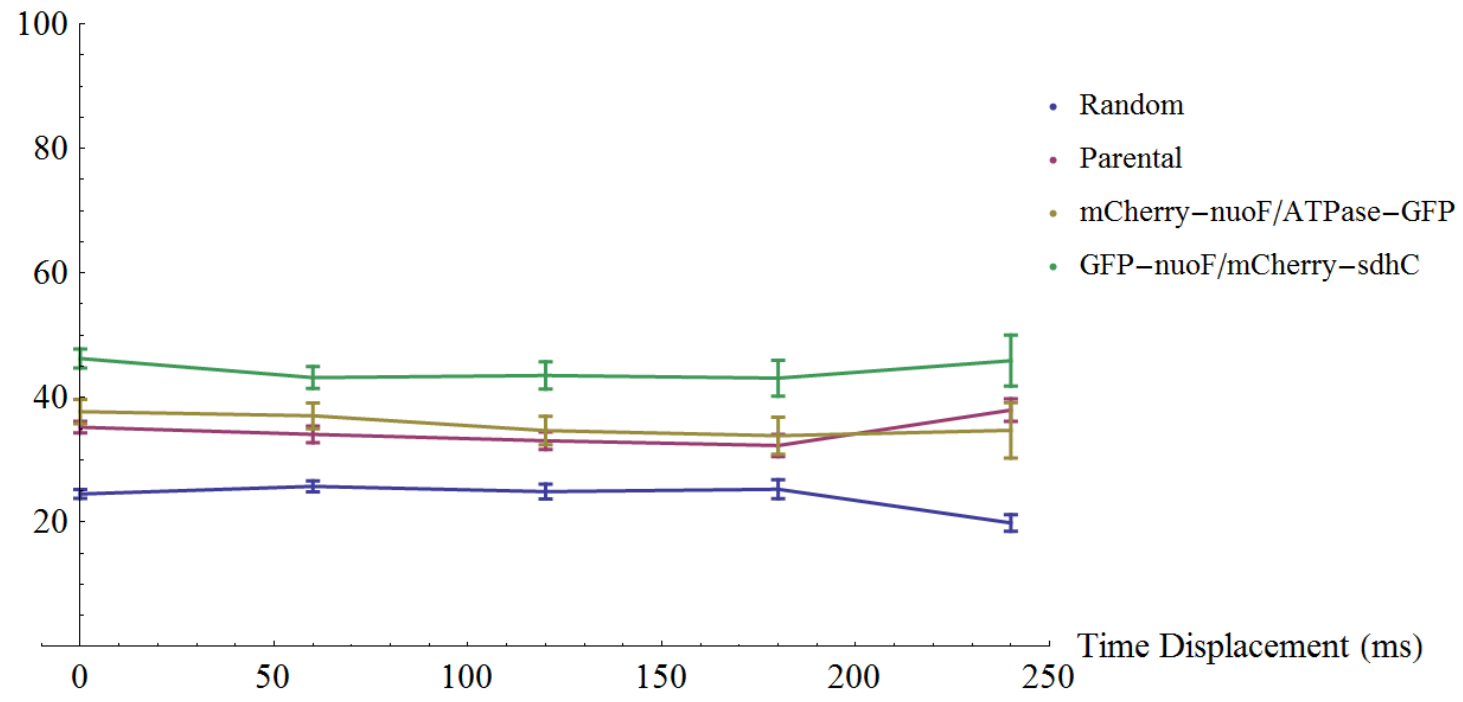

Figure 124 The average correlation values against time displacement for each strain. Each error bar shows \pm the standard error of the mean. Curves for each strain can be identified according to the colour coding as seen in the legend.

\subsection{The Correlation of Each Channel with Itself Displaced In Time}

From the inter-frame self-correlation of single channels, insight was gained into the transience of the fluorescent region distributions. The plots of Figure 125 quantify the average correlation for specific proteins, depending on their displacement in time. In each plot, the strain and fluorescent protein used are indicated by the line style and colour. Dashed and continuous lines indicate the data is from a single-labelled and double-labelled strain respectively. Red lines correspond to the use of mCherry and green to GFP as a marker. 


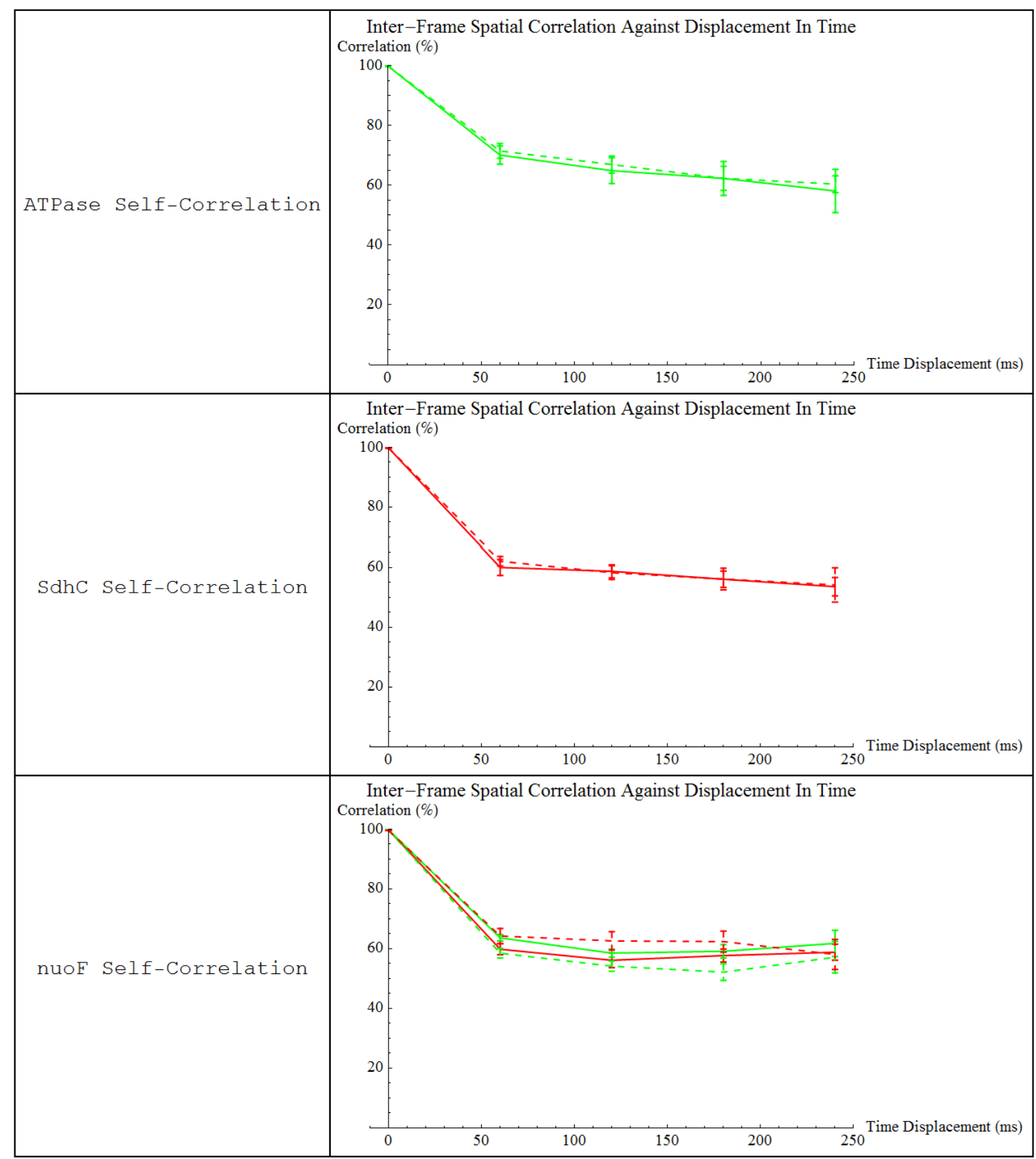

Figure 125 Plots showing the self-correlation of each protein of interest for each cellular context as indicated by the colour and style of line. Red and green indicate data for a protein when labelled with mCherry and GFP respectively. Solid and dashed lines indicate whether the protein was labelled within a double or single labelled strain respectively. Each error bar shows \pm the standard error of the mean. 
For each protein type, the plots are consistent regardless of the strain and label used to obtain the data. With the same labels, very similar results were generated for each protein when present in a single-labelled and double-labelled strain. Also, there is strong agreement in the results for NuoF labelled with mCherry and GFP. These consistencies suggest that the mapping of molecular distribution was not confounded by the labelling and imaging techniques.

\subsubsection{SUMMARY AND CONCLUSIONS}

The chain of reasoning and results in this investigation can be summarised as follows:

Knowledge of the simultaneous spatial distributions of functionally linked OXPHOS proteins in living E. coli would be of benefit to those wishing to better understand the mechanisms of ATP generation and membrane organisation. This knowledge could potentially be gained using TIRF microscopy on a selection of bacterial strains generated by collaborators with genomically encoded fluorescent proteins linked to NuoF, SdhC and ATPase. Experimentation should be tailored to produce the clearest possible individual images of fluorescence distribution, and compromise on other potential forms of single-molecule information such as the generation of particle tracks for diffusion and stoichiometry information already generated within my group.

For each strain, ten videos were recorded and five were selected for further analysis based on the ability to make out diffraction limited features of high intensity within the cell images. This subsampling of data should be considered in the experimental conclusions. Key to the ability to map molecular distribution in a 
membrane is the ability to distinguish features of signal from features of noise. Comparison of cell intensity enabled distinction between un-labelled and labelled strains, but could not enable distinction of labelled strains. The labelling of strains was classified through inspection of image features with a success rate of $82 \%$ and $54 \%$ in the mCherrynuoF/ATPase-GFP and GFP-nuoF/mCherry-sdhC strains respectively. The difficulty to distinguish signal and noise in the GFP-nuoF/mCherry-sdhC strain should lower the confidence level in the results for this strain.

Low-pass spatial filtering of the raw images would smooth intensity variations associated with noise with little effect on features greater and equal in size to the PSF of the imaging system. Rescaling of all cell images to a common range of values would lose information on absolute intensity but would enable comparison of regions of relatively high and low intensity for each cell. Combination of the monochrome images from each colour channel into RGB images produced single images in which the correlation of fluorescence could be discerned with a precision of $\sim 70 \mathrm{~nm}$ (the object width seen in each pixel). These images made it apparent that NuoF and ATPase, and NuoF and SdhC could be both colocalised and not colocalised within a cell. All further work was aimed at determining whether the fluorescence correlation could be attributed to the activity of molecules following independent or dependent trajectories.

For an automated analysis of image correlation, the cell images were binarised with a threshold value equating to the upper quartile of their pixel values. This step lost pixel intensity resolution but enabled generation of a metric for correlation that would be consistent across cells. Since a consistent proportion of the cell pixels were given a high value, the results of correlation could be compared with a probabilistic approximation. 
Plots of correlation against time revealed high variability. The GFP-nuoF/mCherry-sdhC strain exhibited a significantly high level of correlation since there was no overlap of its error bars with those of the other strains. This was taken to indicate colocalistaion greater than would have been expected from independent molecular distributions and lend support to the Respirazone hypothesis.

A technique was developed that made use of the mobility within the membrane to provide information on the independence of the protein distributions. The correlation was not only determined between the channel images within each frame, but it was also determined between the channel images from frames displaced in time. It was reasoned, with some exceptions, that if molecules were significantly colocalised, the correlation would drop on average as the time displacement increased. From plots of correlation against time displacement, comparisons were made between the simulated random, parental, and double-labelled strains. There was no significant drop in correlation in the curves of each strain, indicating independent molecular distributions of NuoF and ATPase, and NuoF and SdhC. This interpretation contrasted with the interpretation of the result that NuoF and SdhC had significantly higher correlation for zero time displacement. The technique of comparing images displaced in time was applied to single channels to provide information on the mobility of the labelled protein types. The correlation vs. time displacement curves for each protein were found to be strongly consistent regardless of the associated fluorescent label or whether the strain was singleor double-labelled.

The apparently independent distributions of NADH dehydrogenase I and ATP synthase support the concept of spatial decoupling of molecules that energise the 
membrane and molecules that use the energy. The contrasting results regarding the independence of the NADH dehydrogenase I and Succinate dehydrogenase distributions leaves open the question of whether the Respirazone hypothesis is applicable to electron transport chains in E. coli.

Though a novel approach (including system-level simulation) was applied to the optimisation of the experiment, I believe the data quality could still be improved significantly. Much optimisation was carried out after the microscope was build, however I think a large gain would be made by rebuilding the microscope with a much more minimal emission path. I have designed a new microscopy layout in which the colour splitting is carried out within the infinity space between the objective and tube lenses. This design would results in a total of two lenses between sample and image sensor rather than the six lenses that were used in collecting the data. This reduction in emission path lenses would reduce signal attenuation and image aberrations. An improved filter set would be experimentally established to further reduce the artefacts of bleedthrough and direct excitation.

With the next generation microscope I would first strengthening the investigation by reproducing and investigating the counterintuitive results of Figure 111. An explanation would be sought for why in some cases a single-labelled cell emitted more strongly in the channel in which it was not labelled. Next I would incrementally improve the experiments to the point where I could distinguish labelled and unlabelled channels (as in Figure 112) with very high accuracy for each strain. Following this it should be possible to assert experimental conclusions with much greater confidence. 


\subsection{The Binding and Dynamics of Epidermal Growth Factor and Epidermal Growth Factor Receptor in Cancerous Human Epithelial Cells}

This chapter summarises progress made in a collaborative single-molecule investigation into live colorectal cancer cells. The project was instigated by the D.Phil. student Dr Charlotte Fournier (MB Bch), who is supervised by Professor Mark Leake of the University of York and Professor Sir Walter Bodmer in the Weatherall Institute of Molecular Medicine, University of Oxford. Dr Fournier came from a context of practicing medicine and teaching human physiology, with the intention of illuminating molecular signalling of live cancer cells as a foundation for understanding of the disease. I joined the investigation during the final eight months of my D.Phil., to help design and carry out single-molecule fluorescence microscopy imaging experiments using the microscope described in section 2 of this thesis.

\subsubsection{BACKGROUND}

\subsubsection{Cell Signalling}

In multicellular organisms, signalling and receptor molecules direct cell proliferation, differentiation and death. The majority of signalling molecules are hydrophilic proteins that bind to a complimentary receptor protein on the surface of cells. Binding will typically induce a conformational change in the intracellular portion of the receptor that activates complex downstream signalling cascades, transducing extracellular 
stimuli to the nucleus. The proliferation, differentiation or death of cells is ultimately induced by the activation or suppression of genes and gene regulatory proteins (Alberts, Johnson et al. 2008).

In this investigation the receptor protein of interest is the epidermal growth factor receptor (EGFR), along one of its many signalling proteins (also known as ligands) epidermal growth factor (EGF). EGF may be synthesised by the same cell upon which it acts (autocrine) or by another cell in the organism (paracrine) and transmitted through the blood stream as well as via diffusion through epithelial tissue. Binding of a ligand to the EGF receptor can lead to the activation of many intracellular molecular pathways. The Ras-Raf-MEK-ERK1/2, STAT3 and STAT5 pathways control proliferation and differentiation, and the PI3K-Akt-mTOR cascade that acts to promote survival and to counter programmed cell death (apoptosis) (Sibilia, Kroismayr et al. 2007). A schematic of the Ras-Raf-MEF_ERK1/2 signalling pathway is presented in Figure 126. 


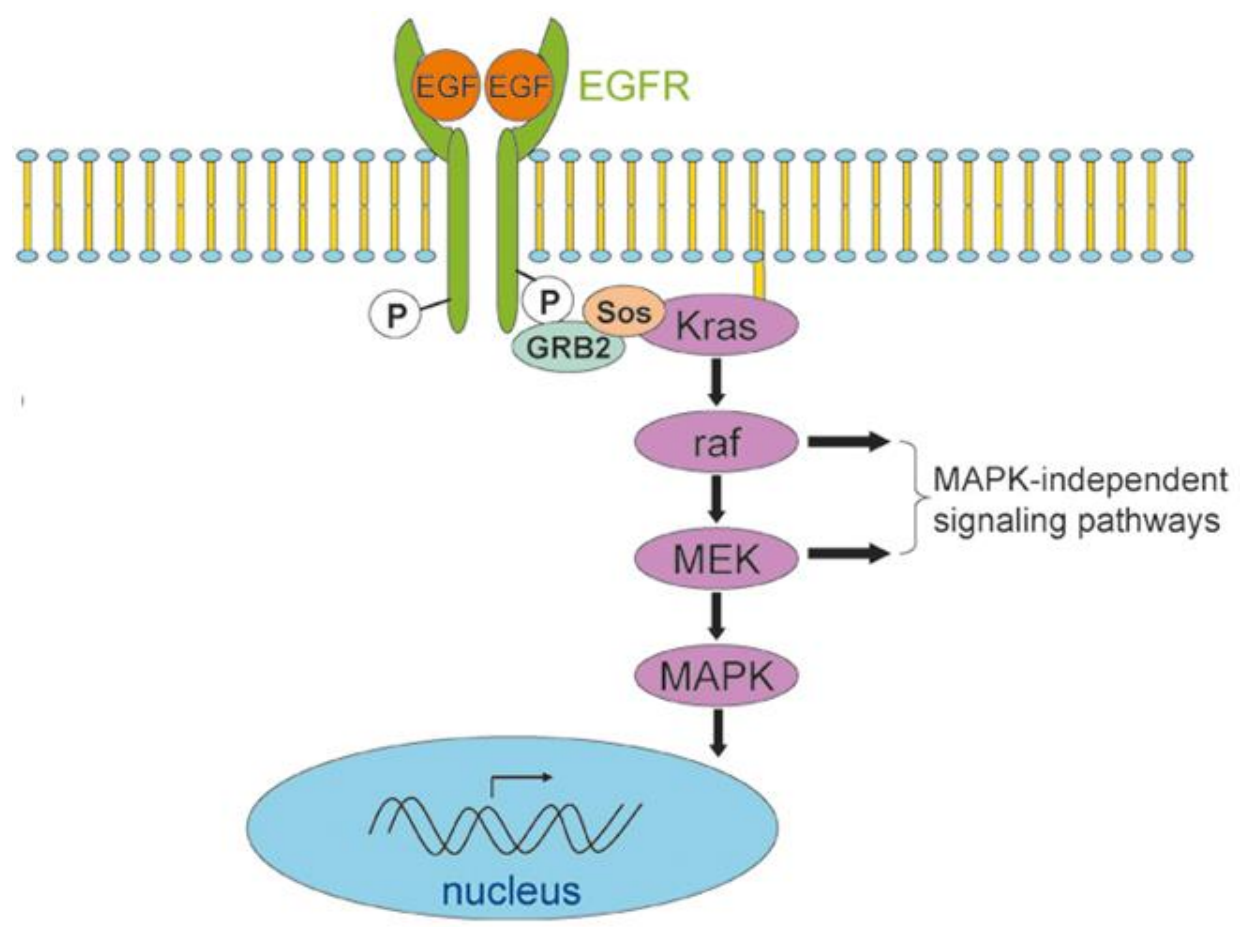

Figure 126 The binding of EGF to the extracellular portion of the EGFR leads to the phosphorylation of gene regulatory complexes in the nucleus, activating gene transcription. Diagram courtesy of Dr Charlotte Fournier, adapted from (Wicki, Herrmann et al. 2010).

\subsubsection{The Role of the EGFR in Development and Cancer}

As an organism develops from the proliferation of a single fertilised egg, cells differentiate and are organised to form different tissues. During normal cell development, autocrine and paracrine signalling results in gene activation and suppression that is appropriate to the stage of developmental and cell function. In the case of cancer, successive mutations in a cell genome enable it and its descendants to escape these normal controls, conferring a selective advantage over neighbouring cells. 
Epithelial cells line tissue surfaces throughout the body and are responsible for functions such as sensing, selective absorption, secretion, and protection. EGF receptor knock-out mice have revealed that during development the EGF receptor controls epithelial cell proliferation and differentiation within several organs, including the skin, lungs, liver and gastrointestinal tract (Miettinen, Berger et al. 1995; Sibilia, Kroismayr et al. 2007).

Deregulation of the EGF receptor is often found in human carcinomas (cancers derived from epithelial cells), that originate in the same organs listed above. In most of these epithelial carcinomas the EGF receptor is overexpressed (Yarden and Sliwkowski 2001; Hynes and Lane 2005). In patients with colorectal cancer, high levels of EGF receptor expression have been associated with the development of carcinomas in distant sites (metastasis) (Spano, Lagorce et al. 2005). Patient survival rates have been improved though treatment with Cetuximab, a monoclonal antibody to the EGF receptor (Yarden and Sliwkowski 2001; Jonker, O'Callaghan et al. 2007) .

Despite the strong link with cancer, the precise mechanism of activation of the EGF receptor has not been elucidated. It has been shown that the EGF receptor exists in a monomeric, dimeric and even oligomeric state in the membrane of Chinese hamster ovary cells that have been genetically modified to express green fluorescent protein (GFP) fused to the cytoplasmic portion of the EGF receptor (Saffarian, Li et al. 2007; Tao and Maruyama 2008).

The A431 carcinoma cell line expresses very high levels of EGF receptor. In this cell line the unliganded EGF receptor has been seen to fluctuate continuously between monomer and dimer states (Chung, Akita et al. 2010; Kawashima, Nakayama et al. 
2010). However, it is not clear whether EGF binds to EGF receptor monomers which then dimerise, or to preformed EGF receptor dimers (see Figure 127 for molecular structures of EGFR in monomer and dimer form). There is some evidence to suggest binding to dimeric EGF receptors in A431 cells (Sako, Minoghchi et al. 2000). Furthermore, following ligand binding, clusters of up to five EGFR have been detected (Webb, Roberts et al. 2008).

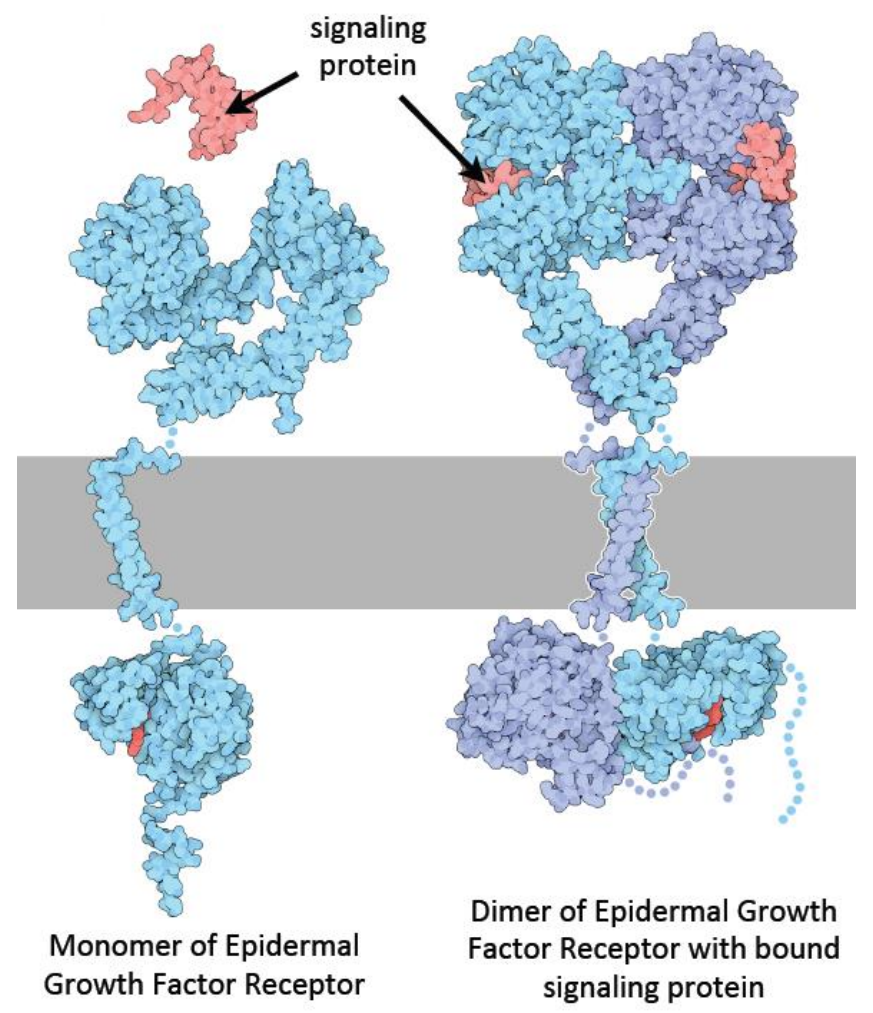

Figure 127 Molecular structures of a monomeric and dimeric EGFR molecules embedded in the cell membrane. Unbound and bound EGF molecules are also displayed. Courtesy of Dr Fournier. 


\subsubsection{Generation of Cell Lines for Fluorescence Imaging}

The aim of the on-going investigation, summarised in this chapter is, to elucidate the mechanism of activation of the EGF receptor by its ligand. In particular, by simultaneously imaging single-molecules of EGF and the EGF receptor, in live cells, to determine whether the ligand binds to a monomeric or dimeric form of the receptor. In order to study the EFG receptor in vivo, a cell line expressing the EGF receptor fused to enhanced green fluorescent protein (EGFP) was generated in the Cancer and Immunogentics Laboratory of Professor Sir Walter Bodmer at the Weatherall Institute of Molecular Medicine.

The Cancer and Immunogenetics Laboratory maintains a panel of over 100 colorectal cancer cell lines. Each cell line expresses mRNA coding for the EGF receptor. The amount of mRNA expressed varies from cell line to cell line, and therefore so does the number of receptors expressed within the cell membrane. The mRNA levels for EGFR in each cell line are plotted in Figure 128. 


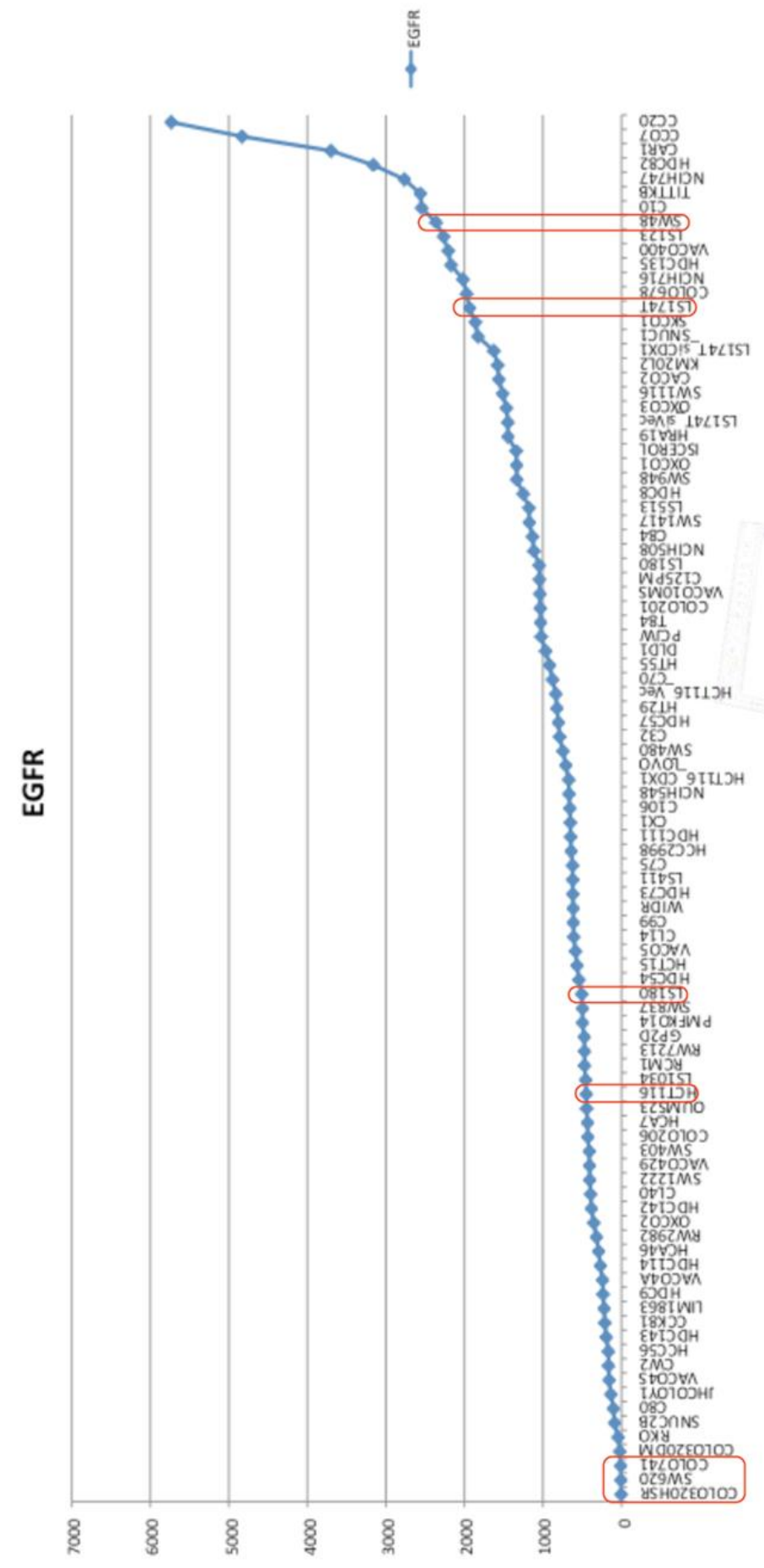

Figure 128 The relative EGFR mRNA expression levels of the panel of colorectal cell lines. Courtesy of Dr Fournier. 
Three cell lines were found to have un-recordable levels of mRNA for EGFR. Through western blotting it was confirmed that these cell lines, COLO741, COLO320HSR, and SW620, did not express the EGFR protein (Figure 129).

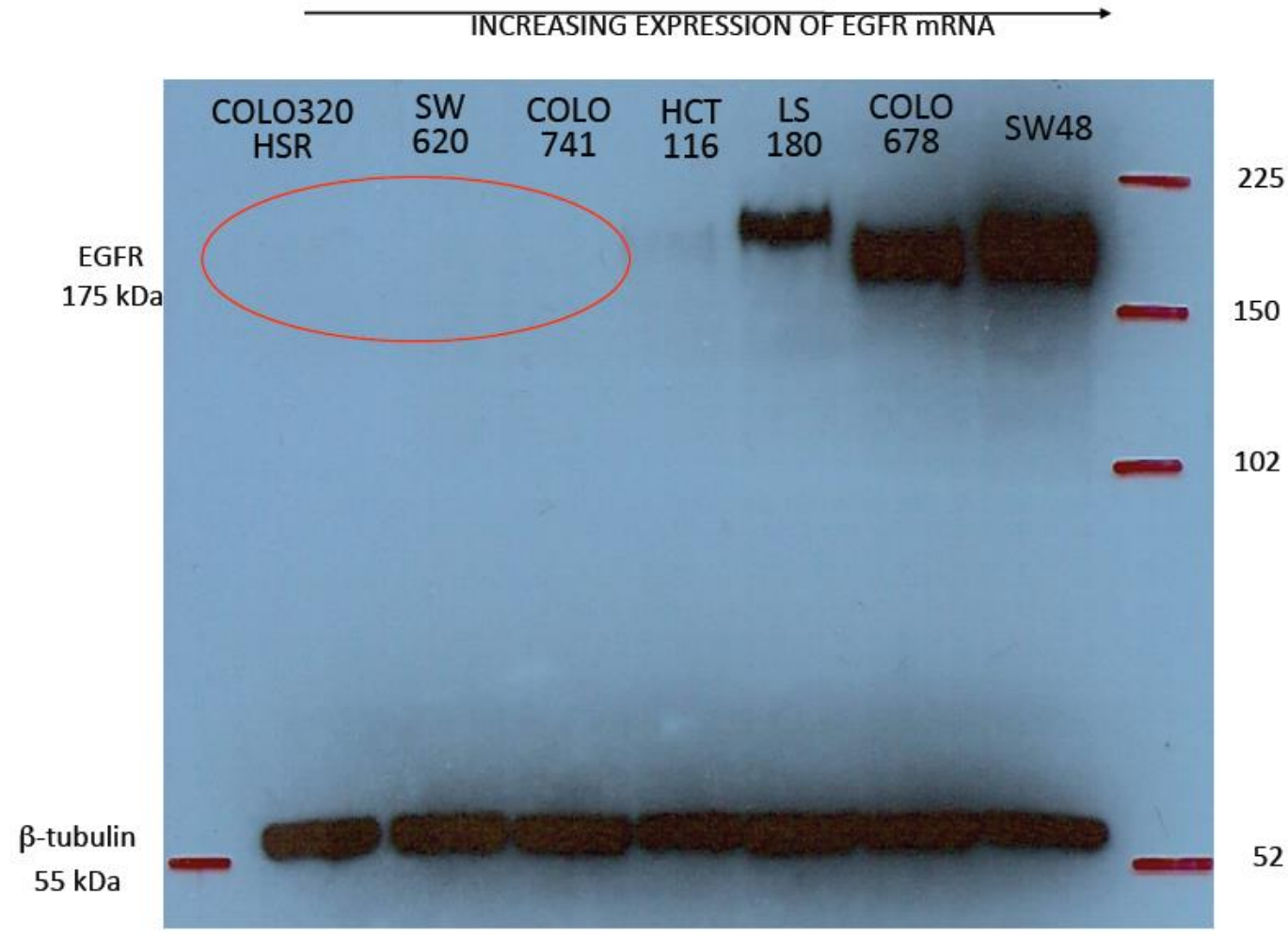

Figure 129 EGFR expression analysed by western blot. Three strains were selected for further analysis due to their lack of EGFR. Figure courtesy of Dr Fournier.

By introducing fluorescently tagged EGFR to a cell line not expressing EGFR, one could carry out quantitative fluorescence imaging experiments with confidence that the complete EGFR population would be accounted for. Though a plasmid sequence for the EGFR attached to EGFP was attained, it was seen to be critical to generate a cell line in which the tagged EGFR would be expressed at physiological levels. The SW620 cell 
line was stably transfected with EGFR (with an EGFP tag attached to the intracellular portion) using Fugene HD transfection reagent and selection with G418.

Confocal fluorescence microscopy images were acquired of the transfected strains. The images demonstrated that the transfected strains had higher levels of green fluorescence than the parental strains. This indicated that indeed the EGFR molecules were tagged. When viewed at high magnification, the green fluorescence appears to be heterogeneously distributed, indicative of clusters of multiple EGFR-EGFP molecules (Figure 130). Furthermore an anti-EGFR tagged with the fluorophore DyLight 633 was found to localise in the same cellular regions as the EGFR-EGFP. In order to obtain quantitative information on the distribution of EGFR-EGFP, single-molecule sensitive TIRF microscopy would be applied. 


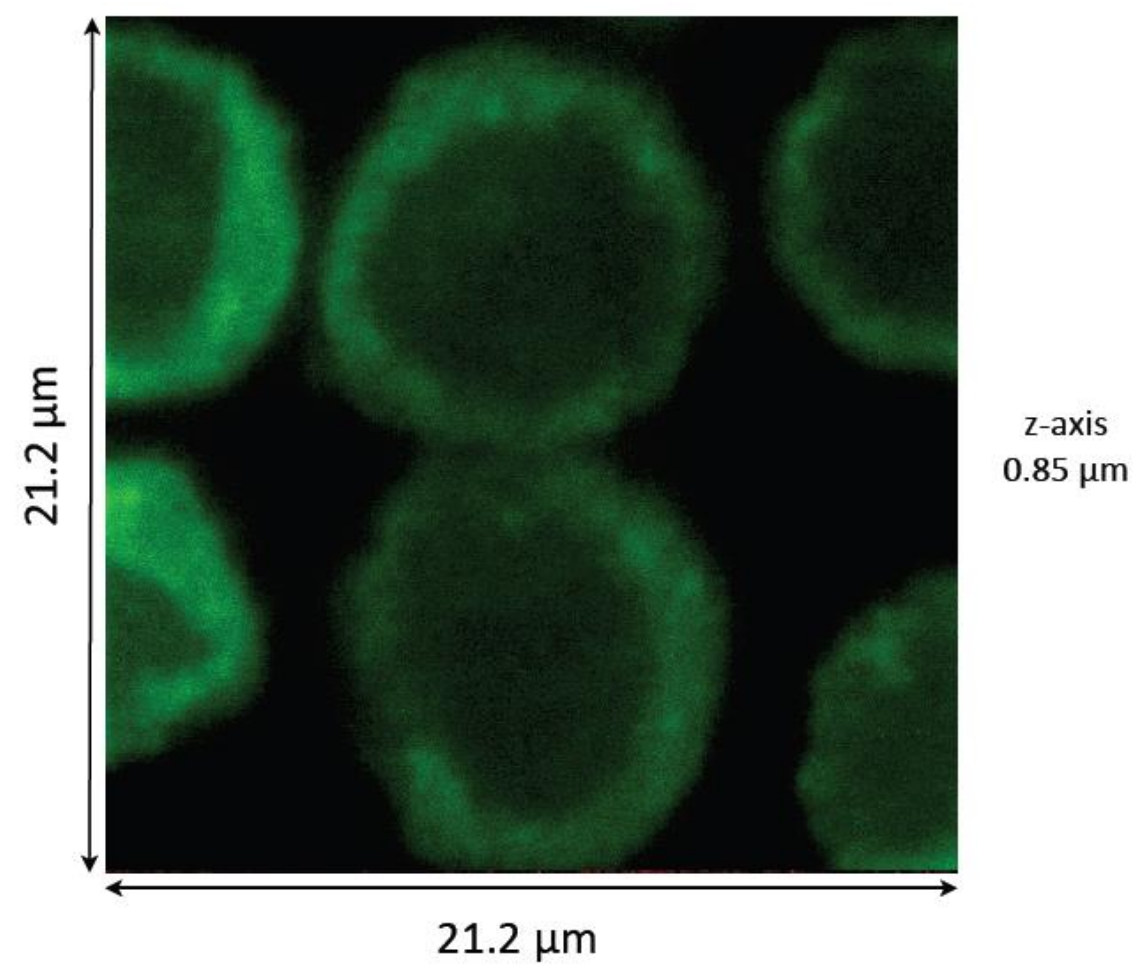

Figure 130 Confocal fluorescence microscopy images of cells transfected to express EGFR-EGFP. A heterogeneous distribution is apparent. Figure courtesy of Dr Fournier.

\subsubsection{Single-Molecule TIRF Imaging of The Cell Lines}

At the time of writing, Dr Fournier is carrying out quantitative analyses on the recorded TIRF microscopy data. Only when these analyses have been completed will experimental conclusions be asserted. What follows is a summary of the experimental design and the data taken, some description of the quantitative analyses, and qualitative description of some intriguing observed phenomena.

Designing the experiments started with an exploration of what questions we hoped to answer and what information could conceivably be attained. The ultimate goal was to determine whether EGFR dimerization occurred prior to or after EGF binding (or 
both). This result would itself be determined from information on the spatial distribution, dynamics, and stoichiometry of imaged fluorophores. The information can be extracted by applying purpose built image analysis software developed by Dr Isabel Llorente Garcia. This software is able to identify fluorescent regions, track them, and produce data for each on brightness and spatial extent with respect to time.

Several modifications were made to the microscope to tailor it to the requirements

of the experiment. Modifications included switching from use of a single $128^{2}$ pixel EMCCD (upon which the smaller bacteria had been imaged) to two $512^{2}$ pixel EMCCDs. One EMCCD would record images of EGFP tagged EGFR molecules while the other would record EGF tagged with the dye tetramethylrhodamine (TMR).

The magnetically mounted splitter unit was switched to correspond to the new camera configuration and appropriate filters were integrated. These filters were selected based on computation of their combined specified transmission spectra, and tested for effectiveness experimentally.

In the excitation path, various beam expansions were experimented with until an expansion was found that was maximally broad (to minimise the variation of intensity across the FOV) but did not diffuse the available laser power too greatly. The angle of incidence of each illumination beam was set to give an evanescent penetration depth of $100 \mathrm{~nm}$.

Imaging of the eukaryotic cells required that a stage top incubator be installed with the ability to maintain physiological condition of $37^{\circ} \mathrm{C}$ and $5 \% \mathrm{CO}_{2}$ concentration. The use of this stage top incubator precluded use of the high NA condenser lens and an 
alternative lens was installed to achieve adequate flux through the sample from a much larger working distance (see section 2.1.3).

Precise focus would be achieved through brightfield illumination of $80 \mathrm{~nm}$ nonfluorescent latex beads, which were apparent even though they were smaller than the microscope point spread function. The image acquisition would be set up and triggered through the purpose built LabView software as in chapter 3.1.

In development of the experiment, control videos were recorded and analysed to identify and minimise the image contribution from each component of the flow cells. The parental cell lines were also imaged, enabling distinction between cellular autofluorescence and the fluorescence from EGFP and TMR.

A protocol (see appendix 5.1.2) was developed to establish a carpet of Fluorescent proteins on the coverslip surface for measurement of the excitation field profile and for measurement of the respective intensities of individual EGFP molecules (see Figure 131 and Figure 132). The surface density could be tailored via the concentration of EGFP solution flowed into the flow cell. The fluorophore of choice could also be modified, and equivalent images were acquired with TMR molecules. 


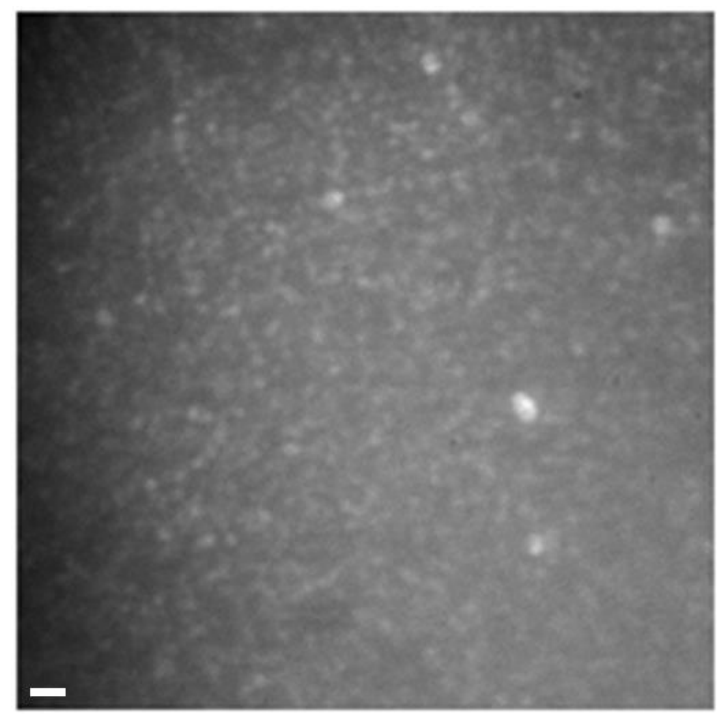

Figure 131 Average image of monomeric EGFP molecules bound to a coverslip surface. $1 \mu \mathrm{m}$ scale bar.

The image analysis software was then applied to such data extract single-molecule and population level information.

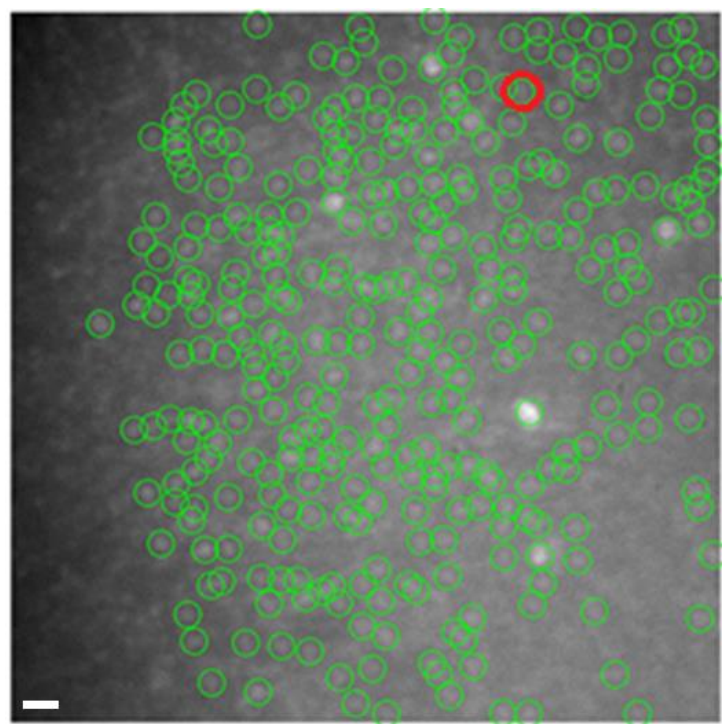

Figure 132 Spots identified by the image analysis software (for a given parameter set) identified with green circles. The spot circled with red is number 244 , the intensity trace of which is presented in Figure 133. $1 \mu \mathrm{m}$ scale bar. 
In the average image images above, the bleaching of fluorophores is not apparent. However, from the video files the integrated pixel intensity of each identified fluorophore could be computed with respect to time. In Figure 133 the intensity trace of a diffraction limited spot is plotted against time. The distinct step in intensity indicates it was a singlemolecule.

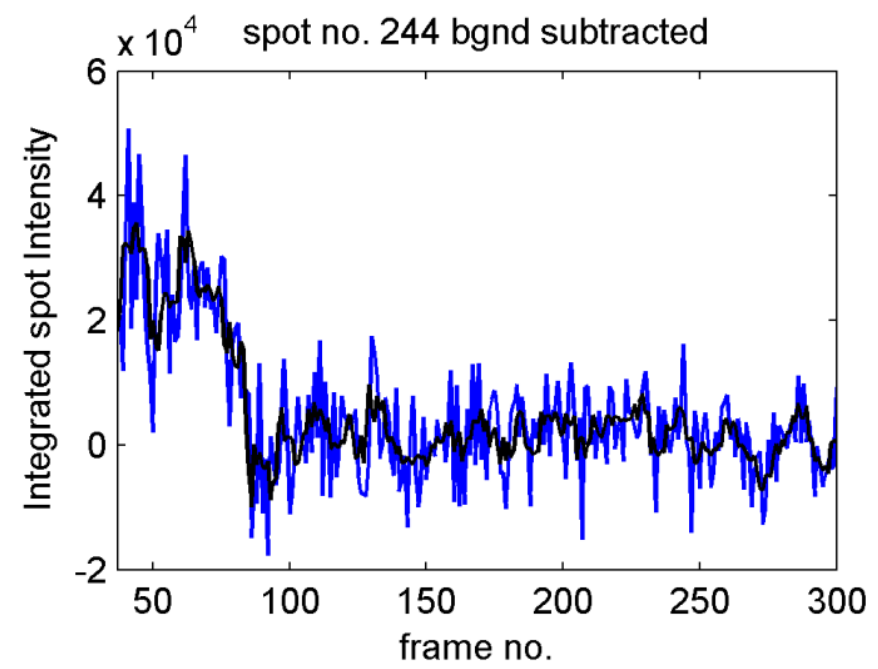

Figure 133 Background subtracted intensity trace of spot 244 as indicated in Figure 132. A step drop to zero in intensity can be seen indicating the quantal nature of fluorescence.

TIRF microscopy videos were recorded of multiple cells prior to and after addition of EGF-TMR at a range of concentrations. A high frame rate was required to capture molecule dynamics and this necessitated a high illumination intensity that would photobleach all fluorophores within ten seconds. Along with phototoxic effects and data storage limitations, this meant that a continuous video could not be taken of each video for the full time period of interest ( one hour). 
To obtain information on the effect of adding EGF over an hour long time period, numerous sequences of videos were taken in which multiple different cells were imaged sequentially following the addition of EGF-TMR to the flow cell. The process of locating, focussing on, and imaging a new cell took approximately five minutes each time. Though there could be cell population heterogeneity and potentially diverse responses to addition of EGF-TMR, it was considered that illuminating trends might appear for videos taken at the same time in different video sequences. At the very least, phenomenological knowledge could be gained from individual videos.

Though the quantitative analyses are incomplete, a qualitative trend did become apparent in the green and red image channels. Prior to addition of EGF-TMR, the EGFREGFP molecules appeared relatively dim and displayed apparent thermal diffusion and some directed motion within the membrane. This situation remained for up to five minutes after adding the ligand, as can be seen in Figure 134.

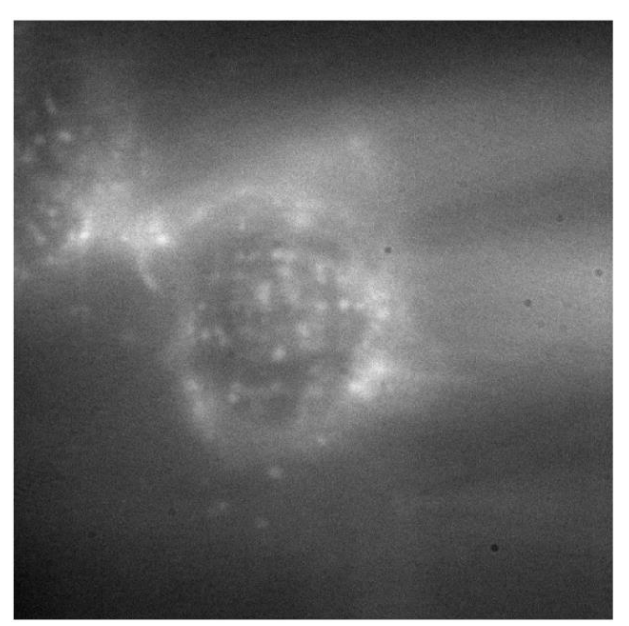

Figure 134 Video frame average of EGFR-EGFP in a live cell, taken five minutes after addition of EGF-TMR. Numerous faint fluorescent spots are evident and appear to move independently through both diffusion and active transport in the membrane. 
From around ten minutes onwards, the fluorescent regions appear to coalesce into a smaller number of relatively bright spots that themselves moved in an apparently directed manner (Figure 135).

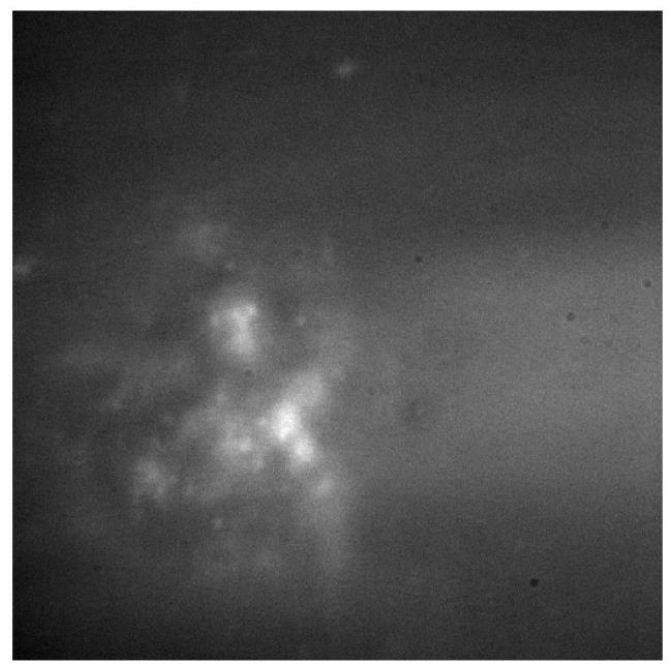

Figure 135 Frame average of a video recorded ten minutes after addition of EGFTMR. The numerous dim fluorescent spots are seen to converge into brighter clusters that themselves follow apparently non-diffusive trajectories.

Twenty minutes after addition of the ligands, the bright spots appeared to be internalised into the cell cytoplasm. This was made apparent by the diffraction rings resulting from bright spots leaving the field of view (Figure 136). Through bright field imaging after video acquisition it was ascertained that the diffraction fringes were not a result of focal drift. 


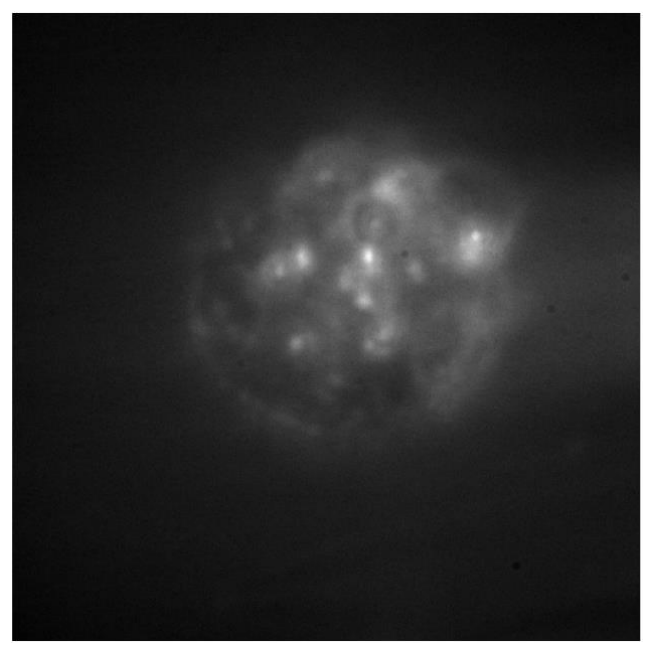

Figure 136 A video frame average of the EGFR-EGFP on a cell taken twenty minutes after addition of EGF-TMR. Diffraction rings are apparent resulting from the spots leaving the focal plane.

It seems most likely that the observed phenomena of EGFR grouping and internalisation into the cell is the process of ligand induced endocytosis (Sorkin and von Zastrow 2009). It has been established that cell signalling and endocytotic membrane trafficking are bidirectionaly linked. It is know that the group of receptors called receptor tyrosine kinases, to which EGFR belongs, undergo endocytosis via clathrin coated pits. This mechanism is used by cells to regulate the activation level of downstream pathways. If the receptors are removed from the cell membrane into a membrane enclosed vesicle (endosome) the concentration of receptors on the cell surface drops and downstream pathway activation is attenuated. 
From ten minutes on, the corresponding red channel videos of the EGF-TMR appeared to show colocalisation of the ligands and receptors. Though at first sight it appears that the addition of EGF results in formation of clusters of multiple EGF and EGFR molecules, the question of whether ligands bind to monomer or dimerised receptors has not yet been answered. The necessary quantitative colocalisation analysis is incomplete. Figure 137 exhibits some quantitative results produced through application of the image analysis software to a live cell video.
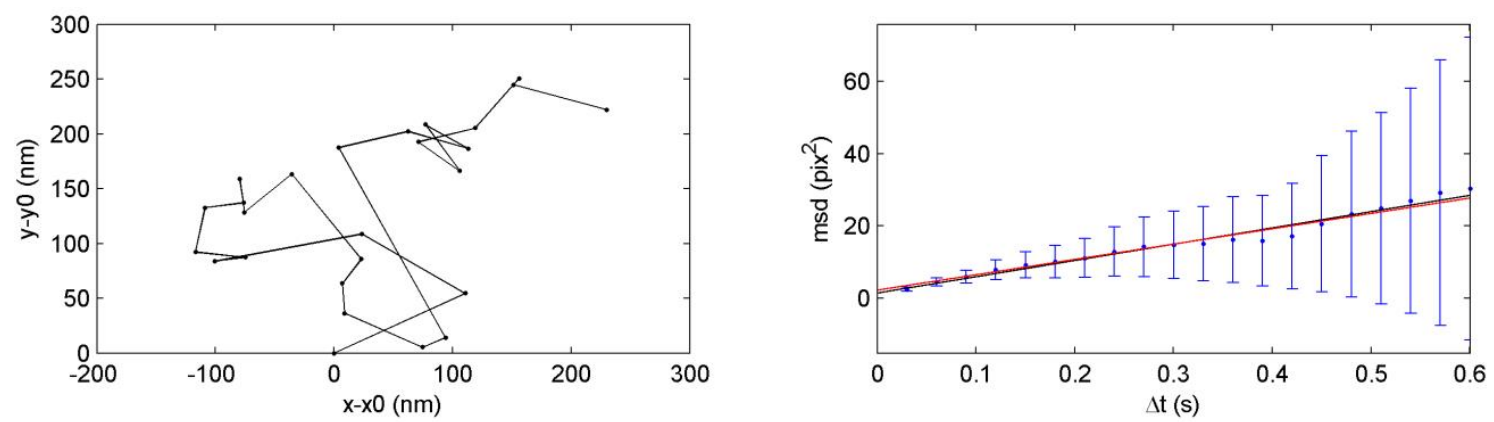

Plots shown are for TRAJECTORY number 5

No. of data points in this trajectory: 26

First frame in this trajectory is: 5

Time origin for sequence is frame no. 1

Time between frames in seconds is: 0.03

No. trajectories analysed: 30

original TRAJECTORY no.: 6

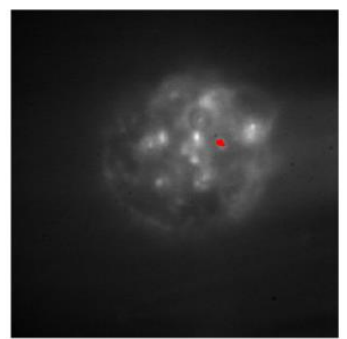

Figure 137 Computational analyses including particle tracking (top left) and statistics on mobility (top right). The fluorescent spot under analysis is indicated by a red spot on the video frame average image (bottom right).

While at this stage no quantitative colocalisation analysis has been completed, some preliminary stoichiometric analysis has been carried out of a sequence of videos where cells were imaged prior to and after the addition of EGF. From the in vitro data 
the average total pixel intensity of each EFGP diffraction limited spot was calculated to be $1888 \pm 853( \pm \mathrm{SD})$. This figure was then compared to the initial total pixel intensity of each tracked spot in the in vivo data. Dividing the initial in vivo intensity values by the average in vitro value approximates and stoichiometry of the in vivo fluorescent spots. The information of interest is how the stoichiometry of EGFR spots changes with respect to the time since addition of the EGF ligand. A plot of this data is presented below in Figure 138.

Stoichiometry of EGFR Clusters Following Addition of EGF Stoichiometry of EGFR Clusters

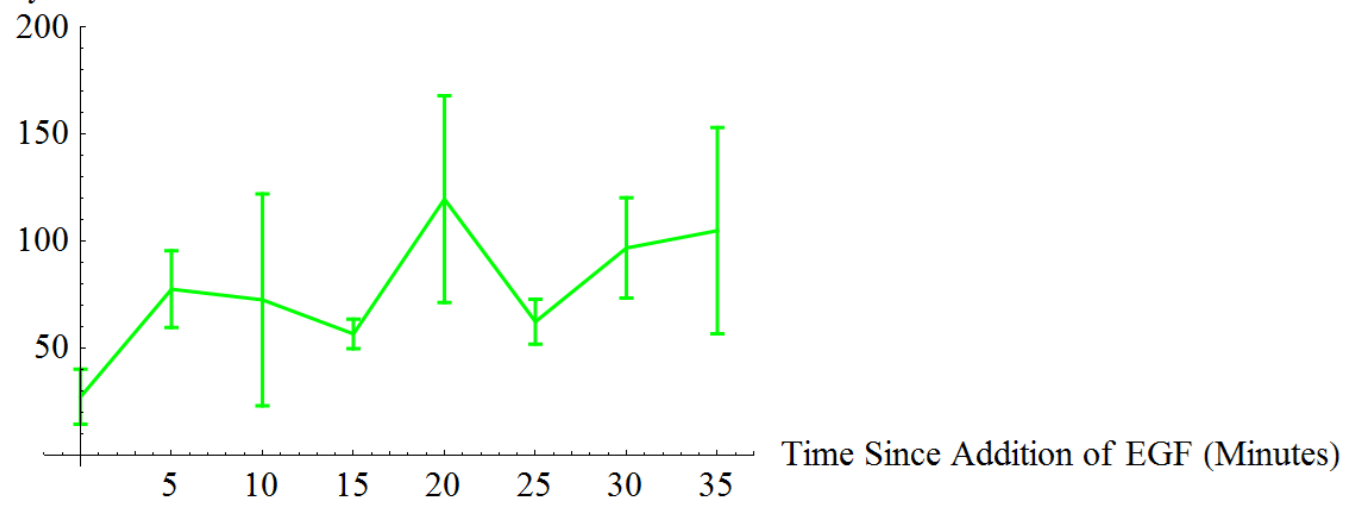

Figure 138 The calculated mean stoichiometry values for the bright spots identified in one sequence of videos. Error bars plotted are \pm the standard deviation of data for each time point. Standard deviation was selected rather than standard error of the mean because in this case the spread of stoichiometry values was of more interest than the confidence in the aggregated mean value.

This analysis suggests that EGFR starts in clusters of around 30 molecules and rapidly coalesces to clusters many times greater with much variability in size. Further analysis is necessary on more video sequences. Furthermore a careful visual inspection the image of each tracked spot must be carried out to verify that the algorithm operated as 
desired. In the cases where spots leave the focal plane and diffraction rings are formed the brightness measured may be inaccurate.

In the course of the experiments brightfield imaging revealed interesting features and activity within the cells. It would have been very informative to have recorded this activity at the same time as the fluorescence activity. However, the spectrum of the halogen lamp used for brightfield illumination precluded simultaneous fluorescence imaging. I identified that an illumination band in the near infrared could enable simultaneous brightfield illumination since it would not excite the fluorophores and the scattered light could be split from the emission beam with a dichroic filter. In future investigations I would integrate the necessary optics to achieve this. 
4 DISCUSSION AND FUTURE OUTLOOK 


\subsection{Reflections on Development of the Microscope}

Having produced a bespoke microscope I have reflected on the necessary sequence of actions and the most rational route to building the instrument. With the benefit of hindsight I have taken into account what information was needed and what needed to be physically established, prior to each progression. From the order of action and information, I have aimed to identify useful heuristics to benefit future development processes.

In the context of experimental science, the most direct route is to start by characterising the sample and to work backwards from the information that is desired. Without a specific measurement in mind, the development process may be ill defined and lengthy. Building an instrument to fulfil multiple measurement types will require disproportionately more work than building for one. In building for multiple users, the number of user inputs to the instrument should be as close to zero as possible.

Effective architectures are primarily enabled by extensive knowledge of the literature. One must have knowledge of the many different ways in which something could be done. Furthermore one must be aware of all the pertinent considerations in order to make well informed designs without bounded rationality. This is gained through experience and can be efficiently built through numerous small projects each limited to one optical concept.

Architectures are most effectively designed freehand where rapidity of ideation is more important than precision. Time spent making a better (e.g. more minimal) architecture is dramatically more effective than on parameter improvements within a given architecture. The principle of keeping options open applies in building also. The 
perceived experimental requirements will change with time and the available knowledge resources and applicable technologies may improve significantly. One should aim to retain the ability to commit to alternative technologies and modify components without perturbing others.

Especially when undergoing a modelling effort, emphasis must be put on minimising the total number of components in the instrument. With fewer components there will be fewer factors that confound understanding, less information and hardware to maintain, lower probability of malfunction or incompatibility, usually fewer optical artefacts, and lower expenditure.

As an alternative to the development of a multifunctional microscope, one could maintain the optical table as a blank sheet with well stocked and ordered laboratory. Minimalist and highly specific instruments would be rapidly designed and built as and when experiments required them.

In building experiments, the measuring component should be established and characterised first. All future work can be evaluated in its effect on the measurements taken. In microscopy, the camera is the measurement component and emphasis should be put on producing high quality images from the outset.

Product Specifications may be accurate but will have subtleties in relevance that must be understood. Though product specification may guide development, every aspect should be judged by its effect on the measurements made. For example, spectral filters should initial be prescribed based on specifications, but only when the desired effect has been measured by the camera should they be settled upon. 
In building and using a complicated instrument the need for multiple experimental iterations is unavoidable. There is a tension between the need to modify one parameter at a time in order to preclude confounding factors, and the benefits of attempting multiple technicalities simultaneously to learn lessons of feasibility and incompatibility in a much shorter time scale (Chin, Linder et al. 2012). To ease this tension a two pronged approach is necessary.

\subsection{Reflections on Simulation of the Experimental System}

The use of a simulation model can reduce some of the complexity of operating an instrument. This can be particularly useful in making experimental calculations, as seen in the case of determining fluorophore brightness and bleaching rates. While the approach can open up new capabilities, it can also introduce unintelligibility into a design and the reliance on an up-to-date model can be seen as an extra burden upon an experimentalist.

Through a validated simulation model it is possible to carry out not only analysis, but also "synthesis", the determination of design from a desired outcome. This approach was demonstrated in the case of reverse ray tracing to inform lens positioning, and the technique for setting desired evanescent field penetration depths.

The system model was found to be particularly useful to a biologist wishing to understand fluorescence microscopy. The relevant parameters were presented in a graphical user interface and the effect of changing them could be explored with feedback

from the simulated images. The code was studied as a structured repository of the 
information seen to be relevant to the experiment, including the abstract physical models that enhance understanding.

Though full refinement and experimental validation was not achieved, with more time it should be possible. Ultimately the time frame in which it is achieved determines the usefulness of the model-aided approach. Since science moves on, the relevance of the model for a specific experimental system may decrease.

Significant time was invested in determining and establishing an effective modelling framework, and in consolidating the necessary experimental information. I see this as the main achievement of the DPhil. Since this has now been achieved, the process takes much less time to apply to further projects. Indeed I recently applied this system modelling approach in the design of a device integrating optics into a microfluidic chip for a successful fellowship application.

The design of a future microscope could be informed by a sensitivity analysis of the system model. The ranking of the system parameters by their effect on system outcomes would guide the trade-offs that are made in the design process. A future microscope would be made in such a way that the three-dimensional form and position of each component was known with high precision. This would be achieved through use of minimal components, each connected through purpose build connectors that precisely meet dimensions based on ray tracing analyses. This would enable more confidence in the in-silico representation of the microscope.

With both a ray tracing model and a model such as the system model in this thesis, and digital position sensing and control of the moving parts, a bi-directionality could be achieved in which changes to the model would result in physical instrument 
changes and vice versa. This would allow for automatic microscope configuration based on the model, and automatic record keeping of the microscope state respectively.

With a minimal, precisely described and controlled system, the process of producing a validated model would become more manageable. Once achieved, computer processing speed and efficient optimisation algorithms would be applied to find optimal imaging conditions. Alternatively, one could take the approach of system synthesis and determine parameter sets capable of achieving a prescribed system performance. This would benefit from simulating the experimental system using a modelling language that enables acausality of information flow. 


\section{BACK MATTER}




\subsection{Appendices}

\subsubsection{TeChNiQUe FOR MiCRometer InCIDENT ANGLE CONTROL}

Follow this procedure each time the microscope is realigned to determine what angles the micrometer values correspond to.

Get the experiment working for TIRF and read the micrometer value to determine the angle of incidence/penetration depth. Attenuate the Gaussian beam so only centroid is visible for safety. Adjust micrometer so the beam travels vertically and strikes the ceiling spot.

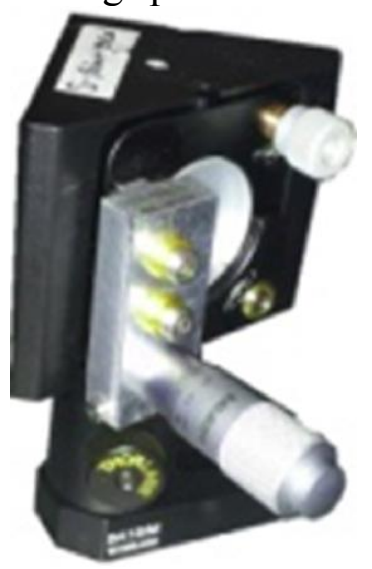

Figure 139 A kinematic mirror mount modified for reproducible orientation with a micrometer.

Put the triangular prism in place so that the edge bisects the vertical beam. Adjust micrometer so the beam is bisected by the upper corner of the prism and record micrometer reading. 


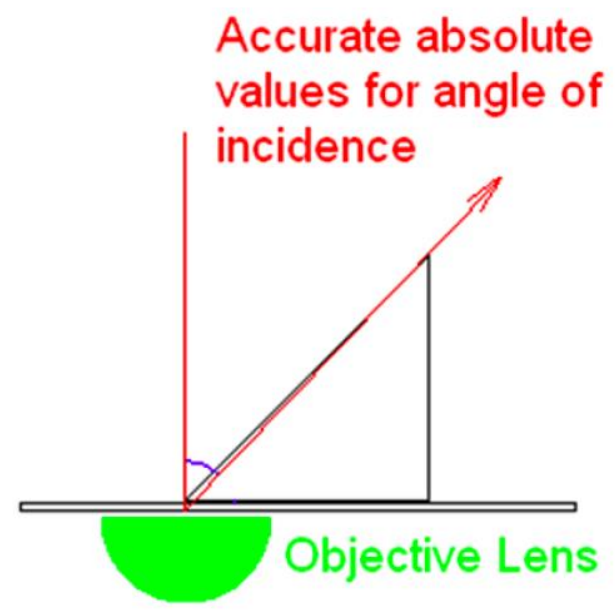

Figure 140 A right-angle prism was used to set the angle of incidence to known value with high accuracy and precision.

Replace the triangular prism with the rectangular prism (composed of stacked slides). Place graph paper soaked with immersion oil on rectangular prism. Set micrometer to vertical value.

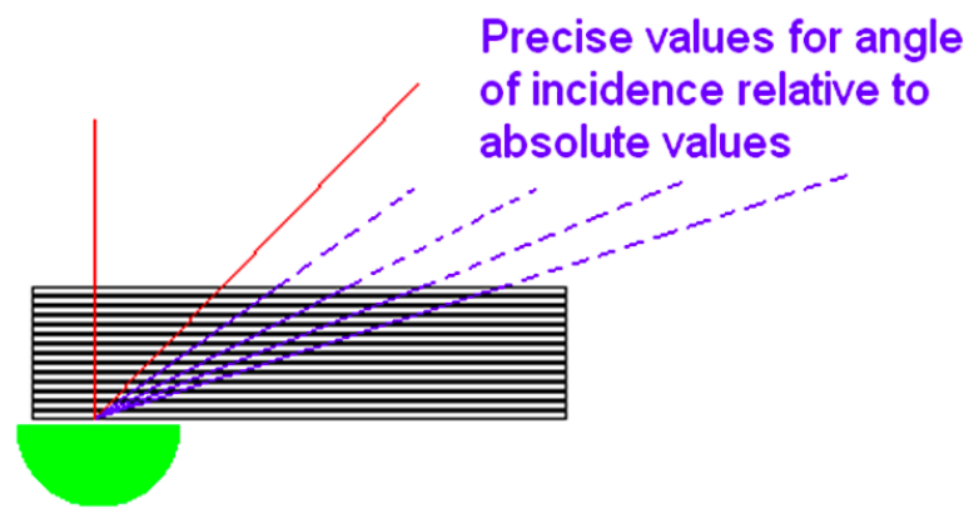

Figure 141 A rectangular prism was to determine the micrometer setting for a series of angles at which total internal reflection was occurring. Multiple slides were stacked with intermediate layers of immersion oil and bound together with silicone adhesive. The incident angles were determined through trigonometry and the use of a strip of graph paper soaked in imersion oil (see figure ). 


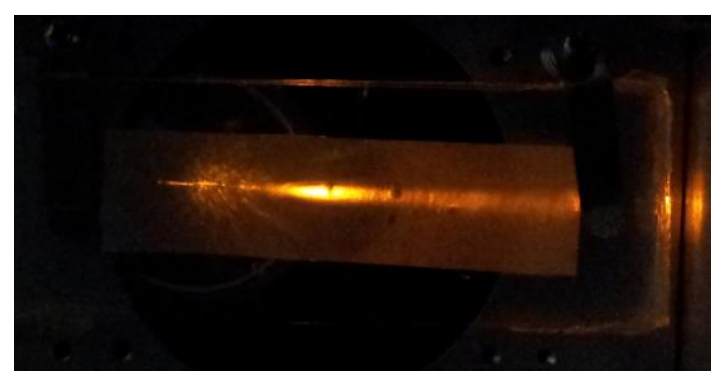

Figure 142 The profile of a beam that totally internally reflects was made evident by placing a strip of paper soaked in immersion oil on the glass surface. Using graph paper enabled measurement of the centroid position for parameterising the model that provides angle of incidence with respect to micrometer position.

Set graph paper to 0 . Set micrometer to value measured at corner of triangle prism (this is actually where beam hits corner of prism, so it's Arctan[25/26]). Take reading on the graph paper. Turn micrometer to get maximum angle of incidence at which beam profile is visible and take micrometer reading. Take readings of micrometer position at the following positions of beam profile on graph paper: $0,5,10,15 \ldots$ to the last multiple of $5 \mathrm{~mm}$ before the beam profile disappears. For example:

$0 \pm 0.25 \mathrm{~mm}$ at 5.15

$5 \pm 0.25 \mathrm{~mm}$ at 4.75

$10 \pm 0.25 \mathrm{~mm}$ at 4.49

$15 \pm 0.25 \mathrm{~mm}$ at 4.33

$20 \pm 0.5 \mathrm{~mm}$ at 4.25

$25 \pm 0.5 \mathrm{~mm}$ at 4.20

$30 \pm 0.5 \mathrm{~mm}$ at 4.16 (30 was coincidentally the last viewable position)

Enter values into Mathematica code and evaluate to produce plots relating micrometer values to incident angles and evanescent penetration depths for each illumination beam. 


\subsubsection{Protocol for In Vitro fluorescent Protein SAMPle Preparation}

This protocol describes how to produce a sample of monomeric EGFP molecules bound to a non-fluorescent coverslip surface using anti-GFP Fab fragments, giving a EGFP surface density of $\sim 1$ per micron ${ }^{2}$ as seen in Figure 132.

1. Construct flow cell from plasma cleaned coverslip, double-sided tape, and 25by- $75 \mathrm{~mm}$ slide.

2. Add $50 \mu 1$ of anti-GFP Fab at $0.2 \mathrm{ng} / \mathrm{ml}$ into each flow cell and incubate for approximately one minute. The anti-GFP Fab was generated using the Pierce Fab Micro Preparation Kit from Thermo Scientific. We used the GFP, ABfinityTM recombinant rabbit monoclonal antibody (G10362) from Invitrogen.

3. Wash through with $100 \mu$ l of phosphate buffered saline (PBS).

4. Flow in $50 \mu 1$ of bovine serum albumin (BSA) at $1 \mathrm{mg} / \mathrm{ml}$ and incubate for 30 minutes.

5. Wash through with $100 \mu$ PBS.

6. Flow in $50 \mu \mathrm{l} \mathrm{EGFP} \mathrm{at} 5 \mu \mathrm{g} / \mathrm{ml}$ and incubate for 5 minutes. The gene sequence for EGFP originates from Clontech Laboratories, Inc.

7. Wash through with $100 \mu$ PBS.

8. Flow in $50 \mu \mathrm{l}$ of $0.5 \mu \mathrm{m}$ latex beads (to enable location of focal plane with brightfield microscopy prior to fluorescence imaging).

9. Wash through with $100 \mu$ PBS. 


\subsubsection{E. Coli Results: AVerage Binarised Images and Correlation against Time Displacement Plots (SeE Sections 3.1.3.3.6 AND 3.1.3.3.7 fOR INTERPRETATION).}

\subsubsection{Simulated Random Strain}

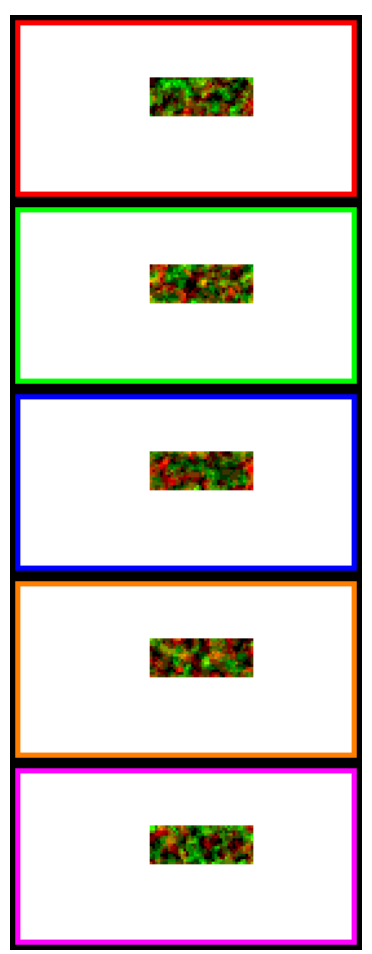

Inter-Frame Correlation Against Displacement In Time Correlation $(\%)$

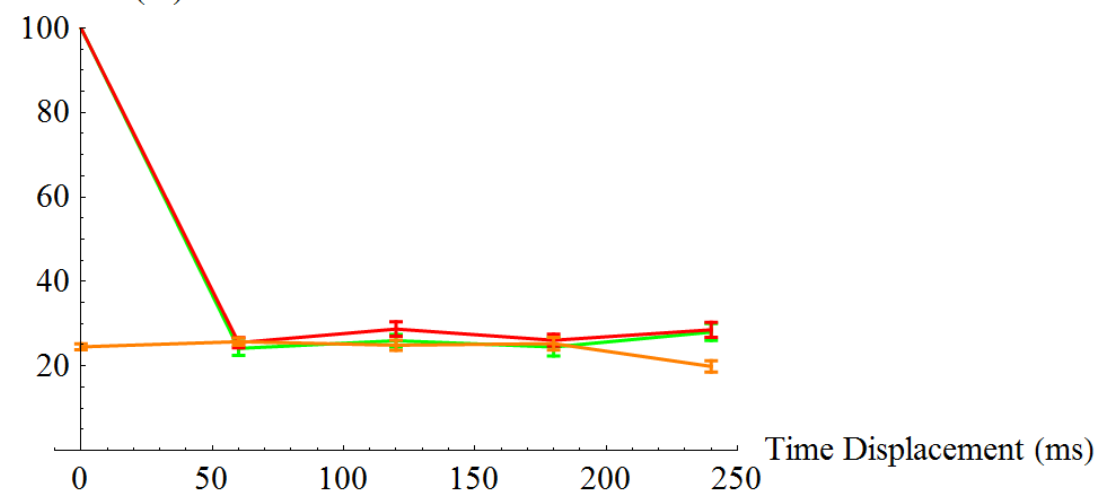




\subsubsection{Parental Strain}

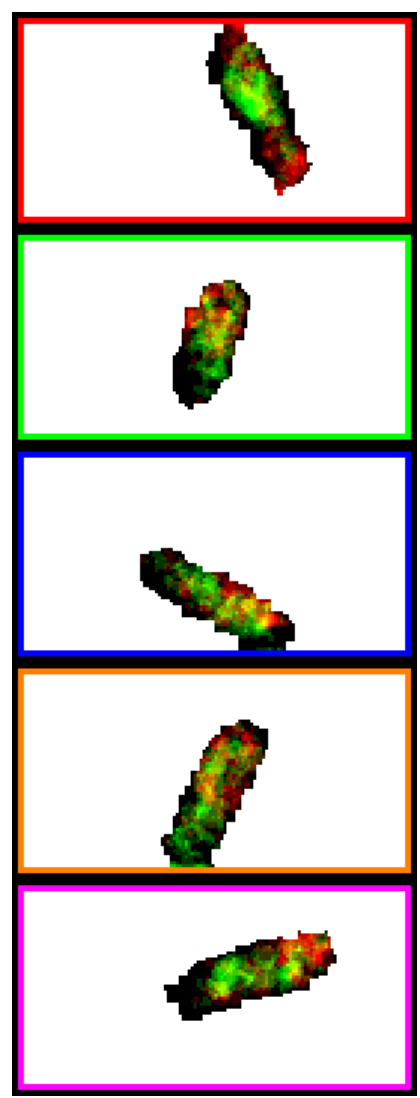

Inter-Frame Correlation Against Displacement In Time Correlation $(\%)$

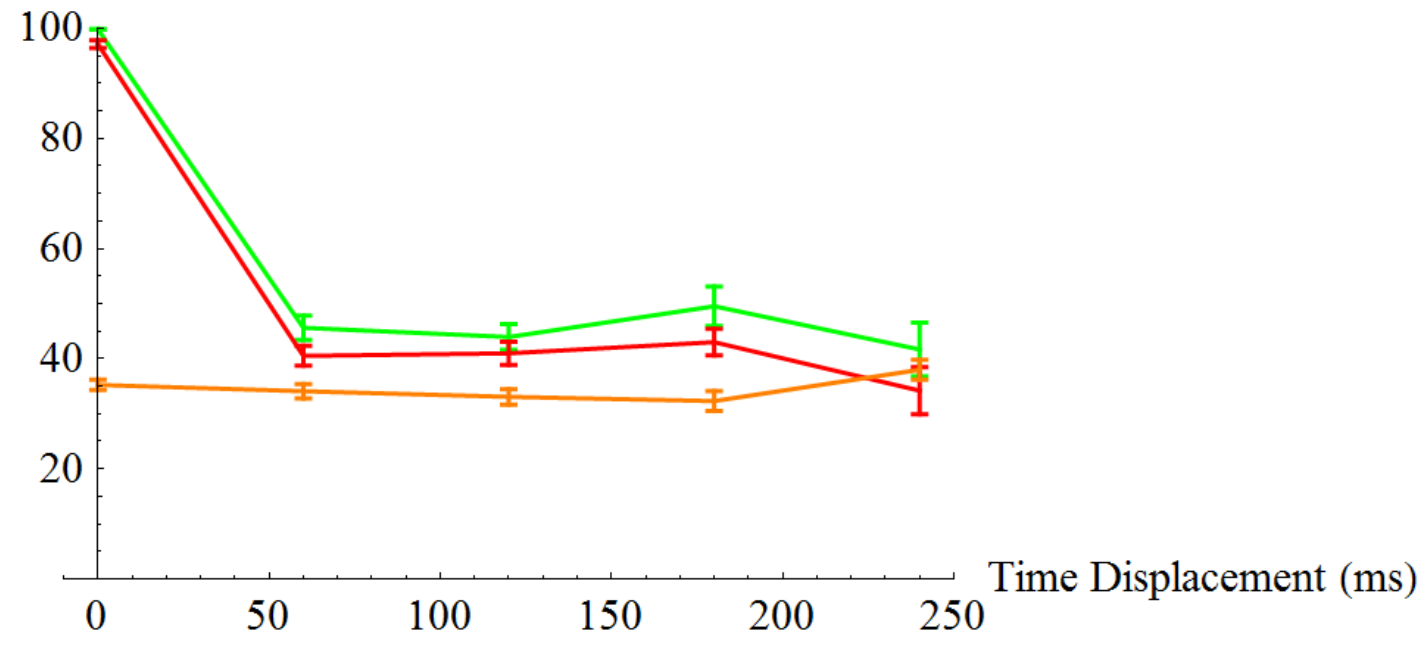




\subsubsection{3 mCherry-nuoF/ATPase-GFP Strain}

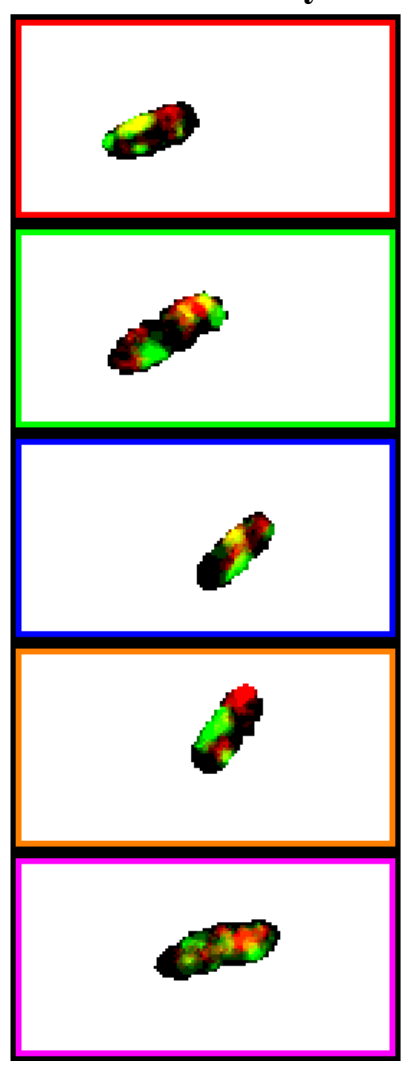

Inter-Frame Correlation Against Displacement In Time Correlation (\%)

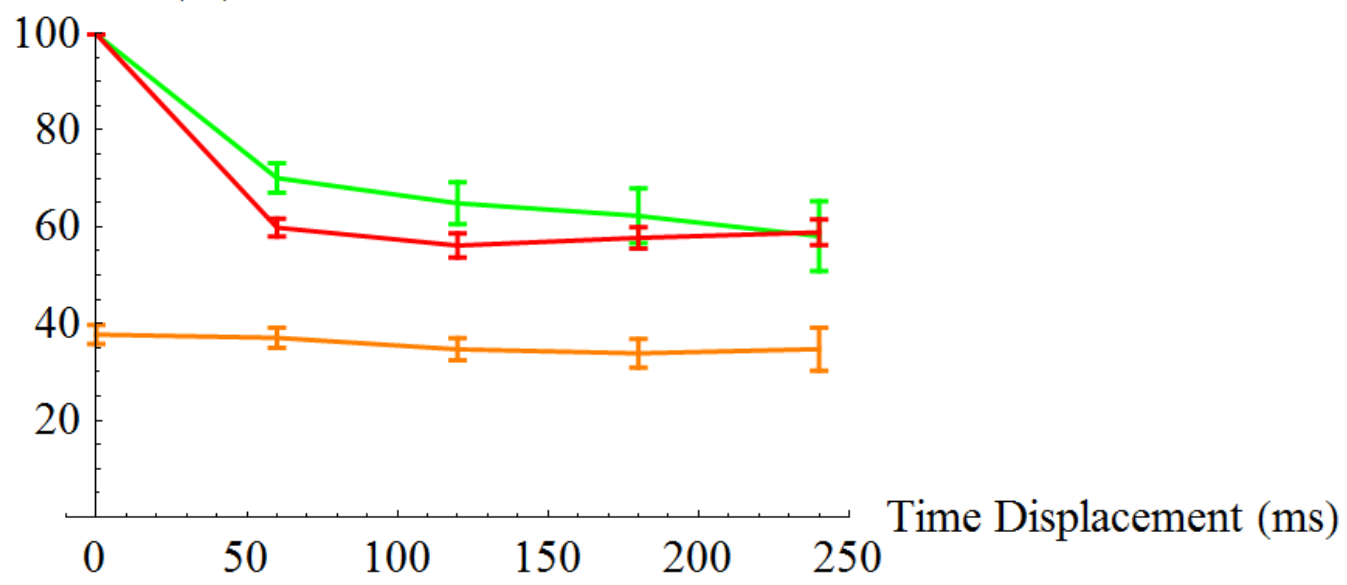




\subsubsection{GFP-nuoF/mCherry-sdhC Strain}

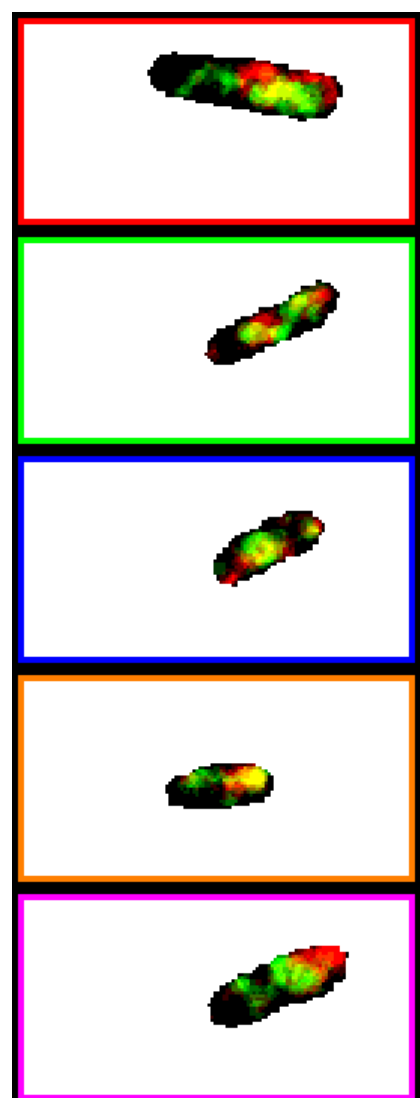

Inter-Frame Correlation Against Displacement In Time Correlation (\%)

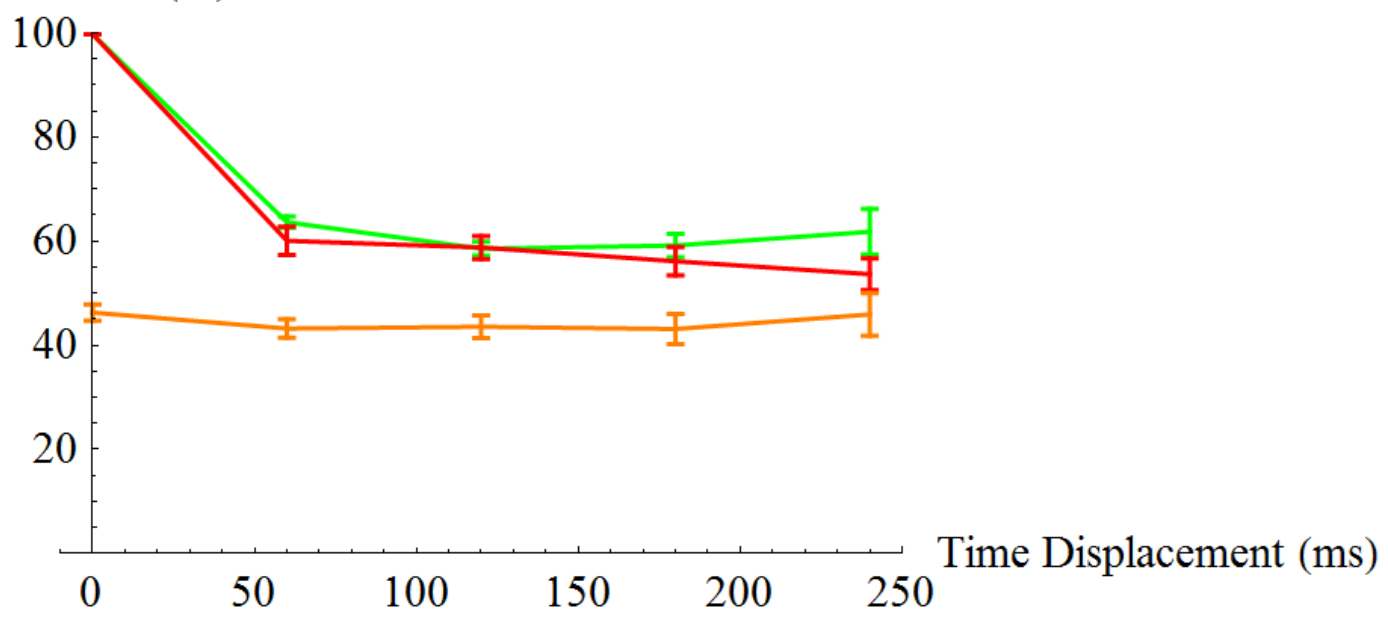




\subsection{References}

Abbondanzieri, E., W. Greenleaf, et al. (2005). "Direct observation of base-pair stepping by RNA polymerase." Nature 438(7067): 460-465.

Agte, J., O. de Weck, et al. (2010). "MDO: assessment and direction for advancementan opinion of one international group." Structural and Multidisciplinary Optimization 40(1): 17-33.

Alberts, B., A. Johnson, et al. (2008). Molecular Biology of the Cell. New York; Abingdon, Garland Science.

Andrews, P., I. Harper, et al. (2002). "To 5D and Beyond: Quantitative Fluorescence Microscopy in the Postgenomic Era." Traffic 3(1): 29-36.

Antony, J. (2003). Design of experiments for engineers and scientists, ButterworthHeinemann.

Armani, A., R. Kulkarni, et al. (2007). "Label-Free, Single-Molecule Detection with Optical Microcavities." Science 317(5839): 783-787.

Ashkin, A., J. M. Dziedzic, et al. (1986). "Observation of a single-beam gradient force optical trap for dielectric particles." Opt. Lett. 11(5): 288-290.

Autumn, K., M. Sitti, et al. (2002). "Evidence for van der Waals adhesion in gecko setae." Proceedings of the National Academy of Sciences 99(19): 12252-12256.

Axelrod, D. (2001). "Total Internal Reflection Fluorescence Microscopy in Cell Biology." Traffic 2(11): 764-774.

Axelrod, D., T. P. Burghardt, et al. (1984). "Total Internal Reflection Fluorescence." Annual Review of Biophysics and Bioengineering 13(1): 247-268.

Badieirostami, M., M. Lew, et al. (2010). "Three-dimensional localization precision of the double-helix point spread function versus astigmatism and biplane." Applied Physics Letters 97(16): 161103.

Bates, M., B. Huang, et al. (2007). "Multicolor Super-Resolution Imaging with PhotoSwitchable Fluorescent Probes." Science 317(5845): 1749-1753.

Bayley, H., T. Luchian, et al. (2008). Single-Molecule Covalent Chemistry in a Protein Nanoreactor. Single Molecules and Nanotechnology. R. Rigler and H. Vogel, Springer Berlin Heidelberg. 12: 251-277.

Binnig, G., C. F. Quate, et al. (1986). "Atomic Force Microscope." Physical Review Letters 56(9): 930-933.

Binnig, G., H. Rohrer, et al. (1982). "Surface studies by scanning tunneling microscopy." Physical Review Letters 49(1): 57-61.

Branton, D., D. Deamer, et al. (2008). "The potential and challenges of nanopore sequencing." Nature Biotechnology 26(10): 1146-1153. 
Bustamante, C., Z. Bryant, et al. (2003). "Ten years of tension: single-molecule DNA mechanics." Nature 421(6921): 423-427.

Chin, C. D., V. Linder, et al. (2012). "Commercialization of microfluidic point-of-care diagnostic devices." Lab on a Chip 12(12): 2118-2134.

Chung, I., R. Akita, et al. (2010). "Spatial control of EGF receptor activation by reversible dimerization on living cells." Nature: 783-U163.

Churchman, S., Z. Â-kten, et al. (2005). "Single molecule high-resolution colocalization of $\mathrm{Cy} 3$ and $\mathrm{Cy} 5$ attached to macromolecules measures intramolecular distances through time." Proceedings of the National Academy of Sciences of the United States of America 102(5): 1419-1423.

Claridge, S., J. Schwartz, et al. (2011). "Electrons, Photons, and Force: Quantitative Single-Molecule Measurements from Physics to Biology." ACS Nano 5(2): 693729.

Colquhoun, D. and A. G. Hawkes (1981). "On the Stochastic Properties of Single Ion Channels." Proceedings of the Royal Society of London. Series B. Biological Sciences 211(1183): 205-235.

Combs, Z., S. Chang, et al. (2011). "Label-Free Raman Mapping of Surface Distribution of Protein A and IgG Biomolecules." Langmuir 27(6): 3198-3205.

da Silva, J., F. Lautenschläger, et al. (2010). "The cavity-to-cavity migration of leukaemic cells through 3D honey-combed hydrogels with adjustable internal dimension and stiffness." Biomaterials 31(8): 2201-2208.

Deniz, A., S. Mukhopadhyay, et al. (2008). "Single-molecule biophysics: at the interface of biology, physics and chemistry." Journal of The Royal Society Interface 5(18): $15-45$.

Deserno, M. (2009). "Mesoscopic Membrane Physics: Concepts, Simulations, and Selected Applications." Macromol. Rapid Commun. 30(9-10): 752-771.

Dietz, H. and M. Rief (2004). "Exploring the energy landscape of GFP by singlemolecule mechanical experiments." Proceedings of the National Academy of Sciences of the United States of America 101(46): 16192-16197.

Dietz, H. and M. Rief (2006). "Protein structure by mechanical triangulation." Proceedings of the National Academy of Sciences of the United States of America 103(5): 1244-1247.

Dietz, H. and M. Rief (2008). "Elastic bond network model for protein unfolding mechanics." Physical Review Letters 100(9).

Dixit, R. and R. Cyr (2003). "Cell damage and reactive oxygen species production induced by fluorescence microscopy: effect on mitosis and guidelines for non invasive fluorescence microscopy." The Plant Journal 36(2): 280-290.

Donato, P. (2012). "Fifty Years of Chemiosmotic Theory-Many Lights and Some Shade." Bioenergetics: Open access.

Downing, K., H. Sui, et al. (2007). "Electron Tomography: A 3D View of the Subcellular World." Analytical Chemistry 79(21): 7949-7957. 
Dudkina, N., R. Kouřil, et al. (2010). "Structure and function of mitochondrial supercomplexes." Biochimica et Biophysica Acta (BBA) - Bioenergetics 1797(67): 664-670.

Egner, A. and S. Hell (2005). "Fluorescence microscopy with super-resolved optical sections." Trends in cell biology 15(4): 207-215.

Eigler, D. M. and E. K. Schweizer (1990). "Positioning single atoms with a scanning tunnelling microscope." Nature 344(6266): 524-526.

Elf, J., G.-W. Li, et al. (2007). "Probing Transcription Factor Dynamics at the SingleMolecule Level in a Living Cell." Science 316(5828): 1191-1194.

Elowitz, M., A. Levine, et al. (2002). "Stochastic Gene Expression in a Single Cell." Science 297(5584): 1183-1186.

Fisher, J. K., M. Ballenger, et al. (2009). "DNA relaxation dynamics as a probe for the intracellular environment." Proceedings of the National Academy of Sciences 106(23): 9250-9255.

French, A., S. Mills, et al. (2008). "Colocalization of fluorescent markers in confocal microscope images of plant cells." Nature Protocols 3(4): 619-628.

Friedenthal, S., A. Moore, et al. (2011). A practical guide to SysML: the systems modeling language, Access Online via Elsevier.

Funatsu, T., Y. Harada, et al. (1995). "Imaging of single fluorescent molecules and individual ATP turnovers by single myosin molecules in aqueous solution." Nature 374(6522): 555-559.

Gao, Z., A. Agarwal, et al. (2007). "Silicon Nanowire Arrays for Label-Free Detection of DNA." Analytical Chemistry 79(9): 3291-3297.

Gautier, A., A. Juillerat, et al. (2008). "An engineered protein tag for multiprotein labeling in living cells." Chemistry \& biology 15(2): 128-136.

Gerlich, D., J. Beaudouin, et al. (2001). "Four-dimensional imaging and quantitative reconstruction to analyse complex spatiotemporal processes in live cells." Nature Cell Biology 3(9): 852-855.

Gordon, M., T. Ha, et al. (2004). "Single-molecule high-resolution imaging with photobleaching." Proceedings of the National Academy of Sciences of the United States of America 101(17): 6462-6465.

Grandbois, M., M. Beyer, et al. (1999). "How strong is a covalent bond?" Science 283(5408): 1727.

Griot, M. (2008). "Gaussian beam optics." Optics Guide, section 2.

Gruebele, M. (2011). "A triple threat to single molecules." Nature Methods 8(3): 213215.

Gupta, P. (2008). "Single-molecule DNA sequencing technologies for future genomics research." Trends in Biotechnology 26(11): 602-611.

Ha, T., T. Laurence, et al. (1999). "Polarization Spectroscopy of Single Fluorescent Molecules." The Journal of Physical Chemistry B 103(33): 6839-6850. 
Harriman, O. L. J. and M. C. Leake (2011). "Single molecule experimentation in biological physics: exploring the living component of soft condensed matter one molecule at a time." Journal of Physics: Condensed Matter 23(50): 503101.

Harriman, O. L. J. and M. C. Leake. (2013). A System Level Approach to Single Molecule Live-Cell Fluorescence Microscopy. infocus, Royal Microscopical Society. 30: 14.

Harvey, W. R. (2009). "Voltage coupling of primary H+ V-ATPases to secondary Na \pm or $\mathrm{K} \pm$ dependent transporters." Journal of Experimental Biology 212(11): 1620-1629.

Hassey, R., E. Swain, et al. (2006). "Probing the Chiroptical Response of a Single Molecule." Science 314(5804): 1437-1439.

Haugland, R. P., M. T. Z. Spence, et al. (2005). The handbook: a guide to fluorescent probes and labeling technologies, Molecular Probes.

Haustein, E. and P. Schwille (2007). "Fluorescence Correlation Spectroscopy: Novel Variations of an Established Technique." Annual Review of Biophysics and Biomolecular Structure 36(1): 151-169.

Hecht, B., B. Sick, et al. (2000). "Scanning near-field optical microscopy with aperture probes: Fundamentals and applications." The Journal of Chemical Physics 112: 7761.

Heintzmann, R., T. Jovin, et al. (2002). "Saturated patterned excitation microscopy?a concept for optical resolution improvement." J. Opt. Soc. Am. A 19(8): 15991609.

Hell, S. and J. Wichmann (1994). "Breaking the diffraction resolution limit by stimulated emission: stimulated-emission-depletion fluorescence microscopy." Opt. Lett. 19(11): 780-782.

Hicks, M., J. Kowalski, et al. (2010). "LD spectroscopy of natural and synthetic biomaterials." Chem. Soc. Rev. 39(9): 3380-3393.

Hilario, J. and S. Kowalczykowski (2010). "Visualizing protein-DNA interactions at the single-molecule level." Current opinion in chemical biology 14(1): 15-22.

Hobot, J., E. Carlemalm, et al. (1984). "Periplasmic gel: new concept resulting from the reinvestigation of bacterial cell envelope ultrastructure by new methods." Journal of bacteriology 160(1): 143-152.

Hoebe, R. A., C. H. Van Oven, et al. (2007). "Controlled light-exposure microscopy reduces photobleaching and phototoxicity in fluorescence live-cell imaging." Nat Biotech 25(2): 249-253.

Hofmann, M., C. Eggeling, et al. (2005). "Breaking the diffraction barrier in fluorescence microscopy at low light intensities by using reversibly photoswitchable proteins." Proceedings of the National Academy of Sciences of the United States of America 102(49): 17565-17569.

Hohlbein, J., C. Joyce, et al. (2011). "Novel Conformational States in Mutator DNA Polymerases Observed Using Single-Molecule FRET." Biophysical Journal 100(3): 240a-241a. 
Hohng, S., R. Zhou, et al. (2007). "Fluorescence-Force Spectroscopy Maps TwoDimensional Reaction Landscape of the Holliday Junction." Science 318(5848): 279-283.

Huang, B., S. Jones, et al. (2008). "Whole-cell 3D STORM reveals interactions between cellular structures with nanometer-scale resolution." Nature Methods 5(12): 10471052.

Huang, B., H. Wu, et al. (2007). "Counting Low-Copy Number Proteins in a Single Cell." Science 315(5808): 81-84.

Hynes, N. and H. Lane (2005). "ERBB receptors and cancer: The complexity of targeted inhibitors (vol 5, pg 341, 2005)." Nature Reviews Cancer: -.

Jain, A., R. Liu, et al. (2011). "Probing cellular protein complexes using single-molecule pull-down." Nature 473(7348): 484-488.

James, W. L. (2012). "Proton-Electrostatics Hypothesis for Localized Proton Coupling Bioenergetics." Bioenergetics: Open access.

Jonker, D. J., C. J. O'Callaghan, et al. (2007). "Cetuximab for the treatment of colorectal cancer." New England Journal of Medicine 357(20): 2040-2048.

Kapanidis, A. and T. Strick (2009). "Biology, one molecule at a time." Trends in Biochemical Sciences 34(5): 234-243.

Kapanidis, A. N., T. A. Laurence, et al. (2005). "Alternating-laser excitation of single molecules." Accounts of chemical research 38(7): 523-533.

Karnovsky, M. J., A. M. Kleinfeld, et al. (1982). "The concept of lipid domains in membranes." The Journal of cell biology 94(1): 1-6.

Kawashima, N., K. Nakayama, et al. (2010). "Reversible Dimerization of EGFR Revealed by Single-Molecule Fluorescence Imaging Using Quantum Dots." Chemistry-a European Journal: 1186-1192.

Kell, D. B. (1992). "The protonmotive force as an intermediate in electron transportlinked phosphorylation: problems and prospects." Curr. Top. Cell. Regul 33: 279289.

Keller, R. (2008). "Green Fluorescent Protein drawn in cartoon style with fluorophore highlighted as ball-and-stick; one wholly-reproduced protein, and cutaway version to show the fluorophore. Modelled and rendered using PyMOL." Wikimedia Commons Public Domain.

Kishino, A. and T. Yanagida (1988). "Force measurements by micromanipulation of a single actin filament by glass needles." Nature 334(6177): 74-76.

Klar, T. A., S. Jakobs, et al. (2000). "Fluorescence microscopy with diffraction resolution barrier broken by stimulated emission." Proceedings of the National Academy of Sciences 97(15): 8206-8210.

Kneipp, K., Y. Wang, et al. (1997). "Single Molecule Detection Using Surface-Enhanced Raman Scattering (SERS)." Physical Review Letters 78(9): 1667-1670. 
Koyama-Honda, I., K. Ritchie, et al. (2005). "Fluorescence Imaging for Monitoring the Colocalization of Two Single Molecules in Living Cells." Biophysical Journal 88(3): 2126-2136.

Kusumi, A., C. Nakada, et al. (2005). "Paradigm shift of the plasma membrane concept from the two-dimensional continuum fluid to the partitioned fluid: high-speed single-molecule tracking of membrane molecules." Annual review of biophysics and biomolecular structure 34(1): 351-378.

Leake, M., J. Chandler, et al. (2006). "Stoichiometry and turnover in single, functioning membrane protein complexes." Nature 443(7109): 355-358.

Lee, H.-1., S. Lord, et al. (2010). "Superresolution Imaging of Targeted Proteins in Fixed and Living Cells Using Photoactivatable Organic Fluorophores." Journal of the American Chemical Society 132(43): 15099-15101.

Lee, S., M. Thompson, et al. (2011). "Super-Resolution Imaging of the NucleoidAssociated Protein HU in Caulobacter crescentus." Biophysical Journal 100(7): L31-L33.

Lenaz, G. and M. Genova (2007). "Kinetics of integrated electron transfer in the mitochondrial respiratory chain: random collisions vs. solid state electron channeling." Am J Physiol Cell Physiol 292(4): C1221-1239.

Lenn, T., M. Leake, et al. (2008). "Are Escherichia coli OXPHOS complexes concentrated in specialized zones within the plasma membrane?" Biochemical Society Transactions 36(5): 1032-1036.

Lenn, T., M. Leake, et al. (2008). "Clustering and dynamics of cytochromebd-I complexes in theEscherichia coliplasma membranein vivo." Molecular Microbiology 70(6): 1397-1407.

Levene, M. J., J. Korlach, et al. (2003). "Zero-Mode Waveguides for Single-Molecule Analysis at High Concentrations." Science 299(5607): 682-686.

Lichtman, J. and J.-A. Conchello (2005). "Fluorescence microscopy." Nature Methods 2(12): 910-919.

Loew, L. M. and J. C. Schaff (2001). "The Virtual Cell: a software environment for computational cell biology." Trends in Biotechnology 19(10): 401-406.

Loparo, J., A. Kulczyk, et al. (2011). "Simultaneous single-molecule measurements of phage T7 replisome composition and function reveal the mechanism of polymerase exchange." Proceedings of the National Academy of Sciences 108(9): 3584-3589.

Lukosz, W. and R. Kunz (1977). "Light emission by magnetic and electric dipoles close to a plane interface. I. Total radiated power." JOSA 67(12): 1607-1615.

Maier, M. (2009). The Art of Systems Architecting, Third Edition (Systems Engineering), CRC Press.

Mandenius, C.-F. and M. Björkman (2011). Biomechatronic Design in Biotechnology: A Methodology for Development of Biotechnological Products, Wiley. 
Manley, S., J. Gillette, et al. (2008). "High-density mapping of single-molecule trajectories with photoactivated localization microscopy." Nature Methods 5(2): 155-157.

Martin, R. (2008). Clean Code: A Handbook of Agile Software Craftsmanship, Prentice Hall PTR.

Mashanov, G. (2003). "Visualizing single molecules inside living cells using total internal reflection fluorescence microscopy." Methods 29(2): 142-152.

Mathies, R., K. Peck, et al. (1990). "Optimization of high-sensitivity fluorescence detection." Analytical Chemistry 62(17): 1786-1791.

McNamara, G., A. Gupta, et al. (2006). "Spectral imaging microscopy web sites and data." Cytometry. Part A : the journal of the International Society for Analytical Cytology 69(8): 863-871.

Mertz, J. (2010). Introduction to optical microscopy, Roberts.

Miettinen, P. J., J. E. Berger, et al. (1995). "Epithelial immaturity and multiorgan failure in mice lacking epidermal growth factor receptor." Nature 376(6538): 337-341.

Millar, N. (2001). "Ligand-Gated Ion Channels." eLS.

Min, W., S. Lu, et al. (2009). "Imaging chromophores with undetectable fluorescence by stimulated emission microscopy." Nature 461(7267): 1105-1109.

Misra, N., J. Martinez, et al. (2009). "Bioelectronic silicon nanowire devices using functional membrane proteins." Proceedings of the National Academy of Sciences 106(33): 13780-13784.

Mitchell, P. and J. Moyle (1967). "Chemiosmotic hypothesis of oxidative phosphorylation." Nature 213: 137-139.

Miwako Mandai, K. Y. (2006). Immersion microscope objective lens, Nikon Corporation.

Mizuno, H., C. Higashida, et al. (2011). "Rotational Movement of the Formin mDial Along the Double Helical Strand of an Actin Filament." Science 331(6013): 8083.

Moerner, W. E. (2002). "A Dozen Years of Single-Molecule Spectroscopy in Physics, Chemistry, and Biophysics." The Journal of Physical Chemistry B 106(5): 910927.

Mortensen, K., S. Churchman, et al. (2010). "Optimized localization analysis for singlemolecule tracking and super-resolution microscopy." Nature Methods 7(5): 377381.

Murphy, D. B. and M. W. Davidson (2013). "Fundamentals of Light Microscopy." Fundamentals of Light Microscopy and Electronic Imaging, Second Edition: 119.

Naik, A. K., M. S. Hanay, et al. (2009). "Towards single-molecule nanomechanical mass spectrometry." Nature Nanotechnology 4(7): 445-450.

Neher, E. and B. Sakmann (1976). "Single-channel currents recorded from membrane of denervated frog muscle fibres." Nature 260(5554): 799-802. 
Nelson, P. C. (2008). "Biological Physics." Freeman, New York.

Neuman, K. and A. Nagy (2008). "Single-molecule force spectroscopy: optical tweezers, magnetic tweezers and atomic force microscopy." Nature Methods 5(6): 491-505.

O'Connor, N. and R. Silver (2007). "Ratio imaging: practical considerations for measuring intracellular $\mathrm{Ca} 2+$ and $\mathrm{pH}$ in living cells." Digital Microscopy, 3rd Edition 81: 415-433.

Opella, S. and F. Marassi (2004). "Structure Determination of Membrane Proteins by NMR Spectroscopy." Chemical Reviews 104(8): 3587-3606.

Pieczonka, N. and R. Aroca (2008). "Single molecule analysis by surfaced-enhanced Raman scattering." Chem. Soc. Rev. 37(5): 946-954.

Plank, M., G. Wadhams, et al. (2009). "Millisecond timescale slimfield imaging and automated quantification of single fluorescent protein molecules for use in probing complex biological processes." Integr. Biol. 1(10): 602-612.

Pohl, T., M. Uhlmann, et al. (2007). "Lambda Red-mediated mutagenesis and efficient large scale affinity purification of the Escherichia coli NADH: ubiquinone oxidoreductase (complex I)." Biochemistry 46(37): 10694-10702.

Preiner, J., A. Ebner, et al. (2009). "Simultaneous topography and recognition imaging: physical aspects and optimal imaging conditions." Nanotechnology 20: 215103.

Rich, P. (2003). "The molecular machinery of Keilin's respiratory chain." Biochemical Society Transactions 31(6): 1095-1106.

Rief, M., M. Gautel, et al. (1997). "Reversible unfolding of individual titin immunoglobulin domains by AFM." Science 276(5315): 1109-1112.

Rissin, D., C. Kan, et al. (2010). "Single-molecule enzyme-linked immunosorbent assay detects serum proteins at subfemtomolar concentrations." Nature Biotechnology 28(6): 595-599.

Riste T, S. D. e. (1990). "Phase Transitions in Soft Condensed Matter." Plenum Press, New York.

Ritort, F. (2006). "Single-molecule experiments in biological physics: methods and applications." Journal of Physics: Condensed Matter 18(32): R531-R583.

Robertson, J., C. Rodrigues, et al. (2007). "Single-molecule mass spectrometry in solution using a solitary nanopore." Proceedings of the National Academy of Sciences 104(20): 8207-8211.

Rowlands, C. J., E. Y. Yew, et al. (2013). "Parallel super-resolution imaging." Nature Methods 10(8): 709-710.

Roy, R., S. Hohng, et al. (2008). "A practical guide to single-molecule FRET." Nature Methods 5(6): 507-516.

Rust, M., M. Bates, et al. (2006). "Sub-diffraction-limit imaging by stochastic optical reconstruction microscopy (STORM)." Nature methods 3(10): 793-795.

Saffarian, S., Y. Li, et al. (2007). "Oligomerization of the EGF receptor investigated by live cell fluorescence intensity distribution analysis." Biophysical Journal 93(3): 1021-1031. 
Sako, Y., S. Minoghchi, et al. (2000). "Single-molecule imaging of EGFR signalling on the surface of living cells." Nature Cell Biology: 168-172.

Sanders, G., G. Dallmann, et al. (2010). "Reconstitution of the B. subtilis Replisome with 13 Proteins Including Two Distinct Replicases." Molecular cell 37(2): 273-281.

SchaĖgger, H. and K. Pfeiffer (2000). "Supercomplexes in the respiratory chains of yeast and mammalian mitochondria." The EMBO Journal 19(8): 1777-1783.

Schutz, G. J., H. Schindler, et al. (1997). "Single-molecule microscopy on model membranes reveals anomalous diffusion." Biophys. J. 73(2): 1073-1080.

Seibert, M., T. Ekeberg, et al. (2011). "Single mimivirus particles intercepted and imaged with an X-ray laser." Nature 470(7332): 78-81.

Semrau, S., T. Idema, et al. (2008). "Accurate Determination of Elastic Parameters for Multicomponent Membranes." Physical Review Letters 100(8): 088101.

Semrau, S. and T. Schmidt (2009). "Membrane heterogeneity - from lipid domains to curvature effects." Soft Matter 5(17): 3174-3186.

Shaner, N., R. Campbell, et al. (2004). "Improved monomeric red, orange and yellow fluorescent proteins derived from Discosoma sp. red fluorescent protein." Nature Biotechnology 22(12): 1567-1572.

Shaner, N., P. Steinbach, et al. (2005). "A guide to choosing fluorescent proteins." Nature Methods 2(12): 905-909.

Sharonov, A. and R. Hochstrasser (2006). "Wide-field subdiffraction imaging by accumulated binding of diffusing probes." Proceedings of the National Academy of Sciences 103(50): 18911-18916.

Shu, X., V. Lev-Ram, et al. (2011). "A Genetically Encoded Tag for Correlated Light and Electron Microscopy of Intact Cells, Tissues, and Organisms." PLoS Biol 9(4): e1001041.

Sibilia, M., R. Kroismayr, et al. (2007). "The epidermal growth factor receptor: from development to tumorigenesis." Differentiation: 770-787.

Sibilia, M., R. Kroismayr, et al. (2007). "The epidermal growth factor receptor: from development to tumorigenesis." Differentiation 75(9): 770-787.

Simons, K. and E. Ikonen (1997). "Functional rafts in cell membranes." Nature 387(6633): 569-572.

Singer, S. J. and G. L. Nicolson (1972). "The fluid mosaic model of the structure of cell membranes." Science (New York, N.Y.) 175(23): 720-731.

Smith, S. B., L. Finzi, et al. (1992). "Direct mechanical measurements of the elasticity of single DNA molecules by using magnetic beads." Science 258(5085): 1122-1126.

Soper, S. A., E. B. Shera, et al. (1991). "Single-molecule detection of Rhodamine 6G in ethanolic solutions using continuous wave laser excitation." Analytical Chemistry 63(5): 432-437.

Sorgenfrei, S., C.-y. Chiu, et al. (2011). "Label-free single-molecule detection of DNAhybridization kinetics with a carbon nanotube field-effect transistor." Nature Nanotechnology 6(2): 126-132. 
Sorkin, A. and M. von Zastrow (2009). "Endocytosis and signalling: intertwining molecular networks." Nature Reviews Molecular Cell Biology 10(9): 609-622.

Sotomayor, M. and K. Schulten (2007). "Single-Molecule Experiments in Vitro and in Silico." Science 316(5828): 1144-1148.

Spano, J. P., C. Lagorce, et al. (2005). "Impact of EGFR expression on colorectal cancer patient prognosis and survival." Ann Oncol 16(1): 102-108.

Stephens, D. and V. Allan (2003). "Light Microscopy Techniques for Live Cell Imaging." Science 300(5616): 82-86.

Strick, T. R., J. F. Allemand, et al. (1996). "The Elasticity of a Single Supercoiled DNA Molecule." Science 271(5257): 1835-1837.

Stroh, C., H. Wang, et al. (2004). "Single-molecule recognition imaging microscopy." Proceedings of the National Academy of Sciences of the United States of America 101(34): 12503.

Taniguchi, Y., P. Choi, et al. (2010). "Quantifying E. coli Proteome and Transcriptome with Single-Molecule Sensitivity in Single Cells." Science 329(5991): 533-538.

Tao, R.-H. and I. N. Maruyama (2008). "All EGF (ErbB) receptors have preformed homo-and heterodimeric structures in living cells." Journal of Cell Science 121(19): 3207-3217.

Thompson, M., J. Casolari, et al. (2010). "Three-dimensional tracking of single mRNA particles in Saccharomyces cerevisiae using a double-helix point spread function." Proceedings of the National Academy of Sciences 107(42): 17864-17871.

Thompson, R. (2002). "Precise Nanometer Localization Analysis for Individual Fluorescent Probes." Biophysical Journal 82(5): 2775-2783.

Tinnefeld, P. and M. Sauer (2005). "Branching Out of Single-Molecule Fluorescence Spectroscopy: Challenges for Chemistry and Influence on Biology." Angewandte Chemie International Edition 44(18): 2642-2671.

Tinoco Jr, I. and C. Bustamante (2002). "The effect of force on thermodynamics and kinetics of single molecule reactions." Biophysical chemistry 101: 513-533.

Tokunaga, M. (1997). "Single Molecule Imaging of Fluorophores and Enzymatic Reactions Achieved by Objective-Type Total Internal Reflection Fluorescence Microscopy." Biochemical and Biophysical Research Communications 235(1): 47-53.

Toprak, E. and P. Selvin (2007). "New Fluorescent Tools for Watching Nanometer-Scale Conformational Changes of Single Molecules." Annual Review of Biophysics and Biomolecular Structure 36(1): 349-369.

Uchihashi, T., R. Iino, et al. (2011). "High-Speed Atomic Force Microscopy Reveals Rotary Catalysis of Rotorless F1-ATPase." Science 333(6043): 755-758.

Unden, G. (1997). "Alternative respiratory pathways of Escherichia coli: energetics and transcriptional regulation in response to electron acceptors." Biochimica et Biophysica Acta (BBA) - Bioenergetics 1320(3): 217-234. 
Valencia-Burton, M., A. Shah, et al. (2009). "Spatiotemporal patterns and transcription kinetics of induced RNA in single bacterial cells." Proceedings of the National Academy of Sciences 106(38): 16399-16404.

van Oijen, A. (1998). "3-Dimensional super-resolution by spectrally selective imaging." Chemical Physics Letters 292(1-2): 183-187.

Vollmer, F. and S. Arnold (2008). "Whispering-gallery-mode biosensing: label-free detection down to single molecules." Nature Methods 5(7): 591-596.

Walter, N., C.-Y. Huang, et al. (2008). "Do-it-yourself guide: how to use the modern single-molecule toolkit." Nature Methods 5(6): 475-489.

Webb, S., S. Roberts, et al. (2008). "Single-molecule Imaging and fluorescence lifetime imaging microscopy show different structures for high-and low-affinity epidermal growth factor receptors in A431 cells." Biophysical Journal: 803-819.

Wicki, A., R. Herrmann, et al. (2010). "Kras in metastatic colorectal cancer." Swiss Med Wkly 140: w13112.

Willcox, K. and O. de Weck (2004). "16.888/ESD. 77 Multidisciplinary System Design Optimization, Spring 2004."

Xie, S., P. Choi, et al. (2008). "Single-Molecule Approach to Molecular Biology in Living Bacterial Cells." Annual Review of Biophysics 37(1): 417-444.

Xie, X. S., P. J. Choi, et al. (2008). "Single-molecule approach to molecular biology in living bacterial cells." Annu. Rev. Biophys. 37: 417-444.

Yang, F., L. Moss, et al. (1996). "The molecular structure of green fluorescent protein." Nature Biotechnology 14(10): 1246-1251.

Yarden, Y. and M. Sliwkowski (2001). "Untangling the ErbB signalling network." Nature Reviews Molecular Cell Biology: 127-137.

Yarden, Y. and M. X. Sliwkowski (2001). "Untangling the ErbB signalling network." Nature Reviews Molecular Cell Biology 2(2): 127-137.

Yildiz, A., J. Forkey, et al. (2003). "Myosin V Walks Hand-Over-Hand: Single Fluorophore Imaging with 1.5-nm Localization." Science 300(5628): 2061-2065.

Yu, J., J. Xiao, et al. (2006). "Probing Gene Expression in Live Cells, One Protein Molecule at a Time." Science 311(5767): 1600-1603.

Zhang, X., E. Settembre, et al. (2008). "Near-atomic resolution using electron cryomicroscopy and single-particle reconstruction." Proceedings of the National Academy of Sciences 105(6): 1867-1872.

Zhuang, X. and M. Rief (2003). "Single-molecule folding." Current Opinion in Structural Biology 13(1): 88-97. 